\title{
Cluster Dynamics Modeling with Bubble Nucleation, Growth and Coalescence
}

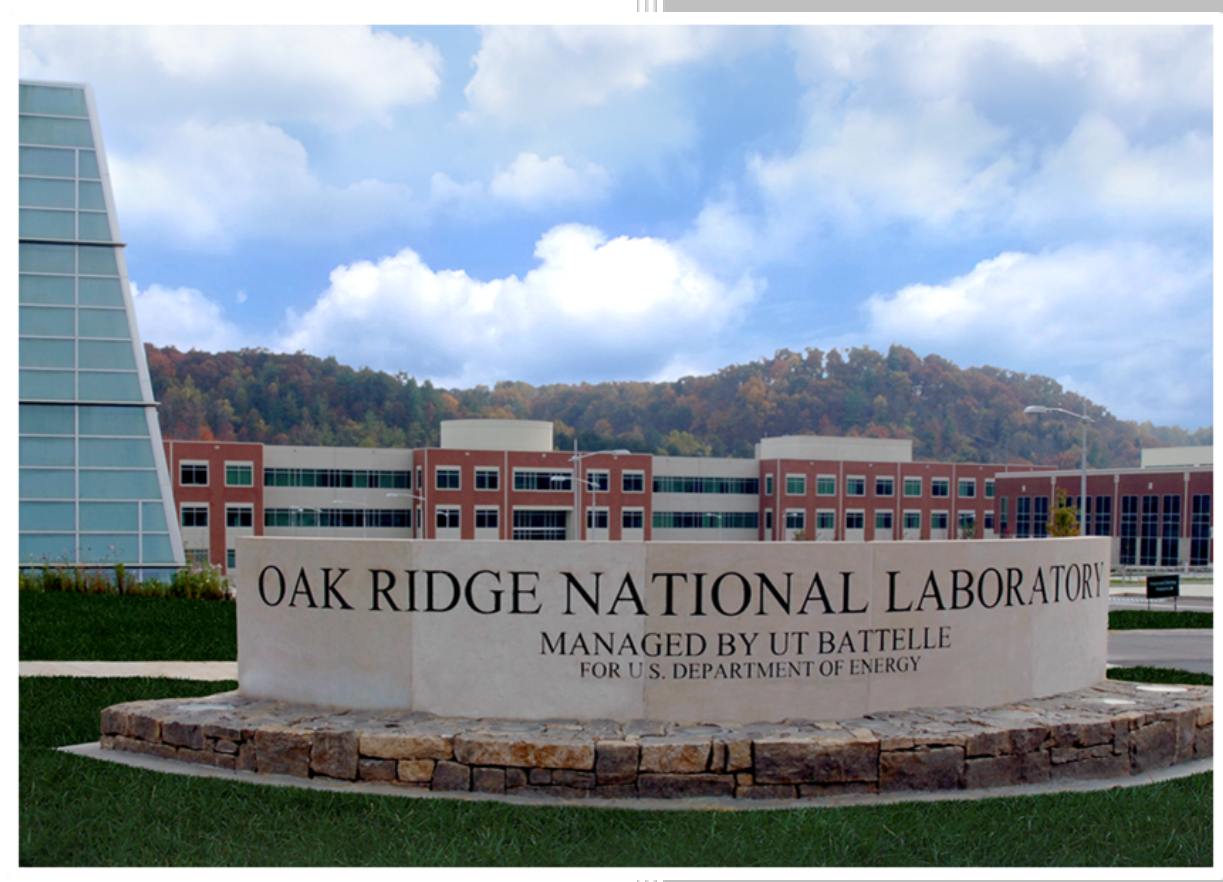

Approved for public release. Distribution is unlimited.

Valmor F. de Almeida Sophie Blondel David E. Bernholdt Brian D. Wirth

17 June 2017 


\title{
DOCUMENT AVAILABILITY
}

Reports produced after January 1, 1996, are generally available free via US Department of Energy (DOE) SciTech Connect.

Website http://www.osti.gov/scitech/

Reports produced before January 1, 1996, may be purchased by members of the public from the following source:

\author{
National Technical Information Service \\ 5285 Port Royal Road \\ Springfield, VA 22161 \\ Telephone 703-605-6000 (1-800-553-6847) \\ TDD 703-487-4639 \\ Fax 703-605-6900 \\ E-mail info@ntis.gov \\ Website http://www.ntis.gov/help/ordermethods.aspx
}

Reports are available to DOE employees, DOE contractors, Energy Technology Data Exchange representatives, and International Nuclear Information System representatives from the following source:

Office of Scientific and Technical Information

PO Box 62

Oak Ridge, TN 37831

Telephone 865-576-8401

Fax 865-576-5728

E-mail reports@osti.gov

Website http://www.osti.gov/contact.html

This report was prepared as an account of work sponsored by an agency of the United States Government. Neither the United States Government nor any agency thereof, nor any of their employees, makes any warranty, express or implied, or assumes any legal liability or responsibility for the accuracy, completeness, or usefulness of any information, apparatus, product, or process disclosed, or represents that its use would not infringe privately owned rights. Reference herein to any specific commercial product, process, or service by trade name, trademark, manufacturer, or otherwise, does not necessarily constitute or imply its endorsement, recommendation, or favoring by the United States Government or any agency thereof. The views and opinions of authors expressed herein do not necessarily state or reflect those of the United States Government or any agency thereof. 
Chemical Sciences Division

\title{
Cluster Dynamics Modeling with Bubble Nucleation, Growth and Coalescence
}

\author{
Valmor F. de Almeida, Sophie Blondel, David E. Bernholdt, and Brian D. Wirth
}

17 June 2017

Prepared by

OAK RIDGE NATIONAL LABORATORY

Oak Ridge, TN 37831-6283

managed by

UT-BATTELLE, LLC

for the

US DEPARTMENT OF ENERGY

under contract DE-AC05-00OR22725 


\title{
Cluster Dynamics Modeling with Bubble Nucleation, Growth and Coalescence*
}

\author{
Valmor F. de Almeida Sophie Blondel ${ }^{\dagger} \quad$ David E. Bernholdt ${ }^{\ddagger}$ \\ Brian D. Wirth ${ }^{\dagger}$ \\ Chemical Sciences Division \\ OAK Ridge National Laboratory \\ OAK RIDGE, TN 37831-6119, USA
}

Technical Report ORNL/TM-2017/322

This manuscript has been authored by UT-Battelle, LLC under Contract No. DE-AC05-000R22725 with the U.S. Department of Energy. The United States Government retains a non-exclusive, paid-up, irrevocable, world-wide license to publish or reproduce the published form of this manuscript, or allow others to do so, for United States Government purposes. The Department of Energy will provide public access to these results of federally sponsored research in accordance with the DOE Public Access Plan(http://energy.gov/downloads/doe-public-access-plan).

17 June 2017

${ }^{\dagger}$ Dept. of Nuclear Engineering, U. of Tennessee at Knoxville

${ }^{\ddagger}$ Computer Science and Mathematics Division

*Also available by request to dealmeidav@ornl.gov 


\begin{abstract}
The topic of this communication pertains to defect formation in irradiated solids such as plasma-facing tungsten submitted to helium implantation in fusion reactor components, and nuclear fuel (metal and oxides) submitted to volatile fission product generation in nuclear reactors. The purpose of this progress report is to describe efforts towards addressing the prediction of long-time evolution of defects via continuum cluster dynamics simulation. The difficulties are twofold. First, realistic, long-time dynamics in reactor conditions leads to a non-dilute diffusion regime which is not accommodated by the prevailing dilute, stressless cluster dynamics theory. Second, long-time dynamics calls for a large set of species (ideally an infinite set) to capture all possible emerging defects, and this represents a computational bottleneck. Extensions beyond the dilute limit is a significant undertaking since no model has been advanced to extend cluster dynamics to non-dilute, deformable conditions. Here our proposed approach to model the non-dilute limit is to monitor the appearance of a spatially localized void volume fraction in the solid matrix with a bell shape profile and insert an explicit geometrical bubble onto the support of the bell function. The newly created internal moving boundary provides the means to account for the interfacial flux of mobile species into the bubble, and the growth of bubbles allows for coalescence phenomena which captures highly non-dilute interactions. We present a preliminary interfacial kinematic model with associated interfacial diffusion transport to follow the evolution of the bubble in any number of spatial dimensions and any number of bubbles, which can be further extended to include a deformation theory. Finally we comment on a computational front-tracking method to be used in conjunction with conventional cluster dynamics simulations in the non-dilute model proposed.
\end{abstract}




\section{Contents}

List of figures $\quad$ iii

\begin{tabular}{lll}
\hline & Introduction & 1
\end{tabular}

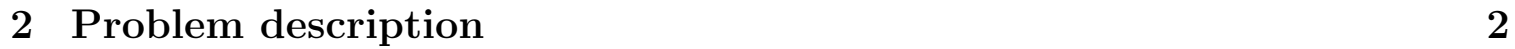

$\begin{array}{lll}3 & \text { Discussion of dilute-limit simulation results } & 3\end{array}$

4 Modeling the non-dilute bubble regime 9

$\begin{array}{lll}5 & \text { Bubble font-tracking } & 13\end{array}$

\begin{tabular}{|lr}
\hline A Appendix: Case $\mathbf{H e}_{240}-\mathbf{V}_{60}$ & 16
\end{tabular}

A.1 Volume fraction plots (linear-linear) . . . . . . . . . . . . . . . . . . 16

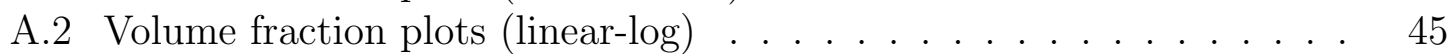

A.3 Cumulative volume fraction plots . . . . . . . . . . . . . . . . . . . . 74

\begin{tabular}{lll}
\hline A Appendix: Case $\mathbf{H e}_{1000}-\mathbf{V}_{250}$ & 103
\end{tabular}

B.1 Volume fraction plots . . . . . . . . . . . . . . . . . . . . . . 103

\begin{tabular}{lr}
\hline Acknowledgments & 216
\end{tabular}

\begin{tabular}{lr}
\hline References & 216
\end{tabular} 


\section{List of Figures}

3.1 Local tungsten void volume fraction, $\phi_{\mathrm{W}}(\boldsymbol{x}, t)$, for the $\mathrm{He}_{240}-\mathrm{V}_{60}$ case at different times in units of $\mu \mathrm{S} \ldots \ldots \ldots 4$

3.2 Local tungsten void volume fraction, $\phi_{\mathrm{W}}(\boldsymbol{x}, t)$, for the $\mathrm{He}_{1000}-\mathrm{V}_{250}$ case at different times in units of $\mu \mathrm{s}$. . . . . . . . . . . . . . . 4

3.3 Penultimate (left) and last (right) species volume fractions, $\phi_{a}(\boldsymbol{x}, t)$,

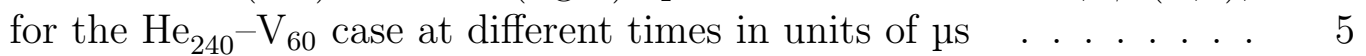

3.4 Penultimate (left) and last (right) species volume fractions, $\phi_{a}(\boldsymbol{x}, t)$, \begin{tabular}{ll|l} 
for the $\mathrm{He}_{240}-\mathrm{V}_{60}$ case at different times in units of $\mu \mathrm{s}$ (log-linear plots) & 5 \\
\hline
\end{tabular}

3.5 Penultimate (left) and last (right) species volume fractions, $\phi_{a}(\boldsymbol{x}, t)$,

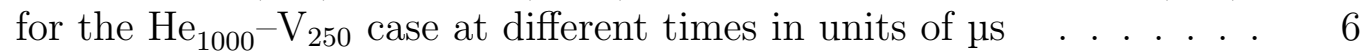

3.6 Penultimate (left) and last (right) species volume fractions, $\phi_{a}(\boldsymbol{x}, t)$, for the $\mathrm{He}_{1000}-\mathrm{V}_{250}$ case at different times in units of $\mu \mathrm{s}$ (log-linear

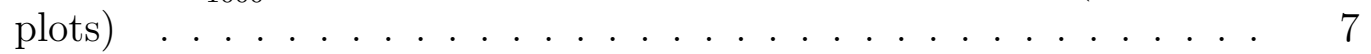

3.7 Example of species helium-vacancy volume fraction, $\phi_{a}(\boldsymbol{x}, t)$, for the

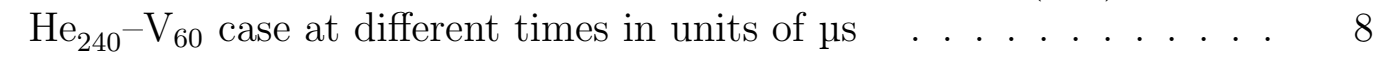

3.8 Example of species helium-vacancy volume fraction, $\phi_{a}(\boldsymbol{x}, t)$, for the $\mathrm{He}_{1000}-\mathrm{V}_{250}$ case at different times in units of $\mathrm{\mu s} \quad \ldots \ldots . . \ldots 8$

4.1 Example of bubble domain at time $t_{0}$, with radius $\boldsymbol{r}\left(t_{0}\right)$, and center $\boldsymbol{x}_{b}\left(t_{0}\right) \ldots \ldots \ldots \ldots \ldots \ldots$

4.2 Bubble domain insertion on all concentration fields at time $t_{0}$, with radius $\boldsymbol{r}\left(t_{0}\right)$, and center $\boldsymbol{x}_{b}\left(t_{0}\right) . \ldots \ldots \ldots \ldots$. . . . . . . . 10

4.3 Sketch of a bubble cut on a relatively small-size cluster at $t_{0}=1 \mu \mathrm{s}$ showing the expected removed section of the concentration curve between red star points as a dotted curve. Case $\mathrm{He}_{240}-\mathrm{V}_{60}$. . . . . . . . 11

5.1 2-D sketch of front-tracking evolution of bubble after nucleation . . . 14

5.2 2-D sketch of front-tracking coalescence of bubbles . . . . . . . . . 14 


\section{Introduction}

ContinuUm modeling of helium implantation in tungsten considered in plasma-

facing components of fusion reactors has employed cluster dynamics in the dilute limit (Faney, 2013). Similar application to fission reactors is the objective of our current work. In both areas, long-time prediction of defect evolution is a necessity since larger structural defects (e.g. voids) of practical interest develop from the complex interaction of underlying smaller defects. The dilute regime assumption of interactions restricts simulated irradiation times to relative short times as compared to reactor operation. Although valuable insight into the behavior of defects can be obtained during the dilute time scale, the continuous influx of guest species into the solid matrix quickly leads to stronger interactions of defects outside the dilute regime. This is certainly the case when long-time evolution leads to, for example, helium-vacancy defects (bubble) nucleation, growth, and eventually coalescence in tungsten components of fusion devices. To the best of our knowledge, no rigorous theory of non-dilute cluster dynamics has appeared, therefore this preliminary work provides insight into the non-dilute regime through simulations in the dilute limit for specific conditions of helium irradiation of tungsten, and explores a path forward towards non-dilute modeling.

Two related open issues challenge our ability to perform realistic, long-time, cluster dynamics simulations in this domain. First, as more incoming He atoms diffuse into the $\mathrm{W}$ matrix, simulation of defect formation calls for a large number of species resulting into a correspondingly large number of concentration fields to be solved for. Ideally, the number of species would be infinite to account for every possible defect formation as time evolves. In practice however, cluster dynamics simulations use a truncated (finite) number of species. The outcome of this simplification is that the nucleation time of the largest helium-vacancy cluster sets an upper bound on the evolution time of a valid simulation. This is so because as soon as this largest cluster nucleates, it grows nonphysically large since no other larger defect is accounted for. Therefore, long-time evolution demands a large number of species to be resolved and this quickly becomes a simulation bottleneck for 3-D domains, say one million concentration fields to reach experimentally accessible irradiation times.

Second, and more importantly, with continued helium implantation and corresponding pointwise concentration increase of individual species with time, the underlying assumption of dilute diffusion regime is no longer valid and the defects have a much stronger, unknown interaction in this spatially crowded condition. Not only the diffusion of the mobile defects under concentrated diffusion regime needs to be properly accounted for, but the interactions of the immobile helium-vacancy clusters with a larger volume fraction (referred as bubbles) need to be addressed. Note that the growth of the largest helium-vacancy cluster in the truncated collection of species

Oak Ridge National Laboratory Report ORNL/TM-2017/322 pp. 1-217 
is an artifact and not an indication of the dilute-to-bubble regime transition (sec. 3).

We are exploring an explicit treatment of geometric defects (bubbles) aimed at capturing non-dilute effects. This approach could set up the stage for a geometric structure of materials with continuous distributions of defects. Specifically, a mechanical theory including defect stress (e.g. Eshelby stress) as the driving force for defect evolution. This is currently missing in the cluster dynamics approach which uses concentration fields of defects (or species) diffusing through a solid matrix. Under dilute conditions, the diffusion can be described by mass balance alone via binary diffusion fluxes. This means cross-term diffusion fluxes are not significant. Once fluxes are higher and defects grow in size, the interactions between species is no longer negligible and a system of configuration forces in a full mechanical theory is needed. Eventually with further developments, a dissipative thermo-mechanical theory for stresses and deformation with internal energy and entropy imbalance considerations could provide the framework for understanding and predicting complex phenomena of helium segregation which often involves swelling of the matrix (Blondel et al., 2017; Maroudas et al., 2016).

We have been inspecting details of simulation results in the dilute regime, and we have demonstrated the localization of concentration profiles for immobile heliumvacancy defects. We argue that the monitoring of the local volume fraction of total helium throughout space provides an indication of non-dilute regime. In addition, the profile shape of individual species, in particular, helium-vacancy defects, provides another indicator to judge when non-dilute conditions are emerging. Finally, observations of the concentration of the largest helium-vacancy cluster gives a clear indication of artifacts present on concentration profiles of other species. Here the adverse effect is to skew the Gaussian shape profile (hallmark of a dilute behavior) of small smaller clusters into a bimodal (or multimodal) shape due to the overlap and rising of the artificial concentration peak for the largest-sized helium-vacancy cluster (truncation effect). These in-depth observations will be further explored in the following sections.

\section{Problem description}

A 1-D test problem was used for detailed observations of the evolution of species concentrations in the dilute regime. The problem is similar to the helium irradiation of tungsten described by Faney (2013) in chapter 6 without the truncation correction (as explained in chapter section 6.2). The Xolotl code was used with model parameters as follows:

- Material surface: W(100)

Oak Ridge National Laboratory Report ORNL/TM-2017/322 pp. 1-217 
- Temperature: $933 \mathrm{~K}$

- Helium flux: $4 \times 10^{7} \mathrm{~nm}^{-1} \mathrm{~s}^{-2}$

- Species types: $\mathrm{He}_{i}, \mathrm{~V}_{j}, \mathrm{I}_{k}, \mathrm{He}_{m}-\mathrm{V}_{n}$

on a uniform mesh with 400 points. Two ordered lists of species were investigated, namely, one list terminating in $\mathrm{He}_{240}-\mathrm{V}_{60}$ (total of $N=602$ species), and another terminating in $\mathrm{He}_{1000}-\mathrm{V}_{250}$ (total of $N=1552$ species). The cases corresponding to these lists will be referred to by the terminating species names. Each species $a$ in the list has a characteristic radius $r_{a}$ and a corresponding volume $v_{a}$ which determines the species volume fraction field $\phi_{a}(\boldsymbol{x}, t):=v_{a} c_{a}(\boldsymbol{x}, t)$ at each $\boldsymbol{x}$ point and time instant $t$. We computed an exhaustive inventory of volume fraction fields, in particular for all $a=\mathrm{He}_{m}-\mathrm{V}_{n}$ species, and compared their evolution in time to assess non-dilute behavior. In addition, the $\mathrm{W}$ void fraction at each space point and time was computed by the simple sum

$$
\phi_{\mathrm{W}}(\boldsymbol{x}, t):=\sum_{a}^{N} \phi_{a}(\boldsymbol{x}, t),
$$

where $a$ represents each of the species in the ordered list. The pointwise cumulative volume fraction for all species $\Phi_{a}(\boldsymbol{x}, t):=\int_{0}^{\boldsymbol{x}} \phi_{a}\left(\boldsymbol{x}^{\prime}, t\right) d \boldsymbol{x}^{\prime}$ at all times can also be computed to show the retained helium. The model includes a moving boundary (left side of the $x$-axis) to account for 1-D swelling of the tungsten (Blondel et al., 2017).

\section{Discussion of dilute-limit simulation results}

It is observed in both cases $\mathrm{He}_{240}-\mathrm{V}_{60}$ (fig. 3.1) and $\mathrm{He}_{1000}-\mathrm{V}_{250}$ (fig. 3.2) that within the order of one microsecond, a peak of void fraction of about 1 vol \% and width of about $2.5 \mathrm{~nm}$ emerges near the surface of the tungsten. Hence a local non-dilute condition emerges very soon in the irradiation evolution. Certainly, an evolution past $3 \mu \mathrm{s}$ is an unrealistic simulation for any of these two different cases (with different number of defect species) since the peak void fraction is greater than $2 \mathrm{vol} \%$.

A closer look at the evolution of the volume fraction of the last and penultimate species in the ordered list $\mathrm{He}_{240}-\mathrm{V}_{60}$ shows a great disparity in the results for any of the irradiation times (figs. 3.3 and 3.4). Note that the difference in species size amounts to only one He atom, yet the volume fraction profiles are drastically different quantitatively and qualitatively. The peak of the volume fraction of the $\mathrm{He}_{239}-\mathrm{V}_{60}$ species does not emerge until after $0.71 \mu \mathrm{ss}$ (fig. 3.4, left plot) while the corresponding volume fraction for the $\mathrm{He}_{240}-\mathrm{V}_{60}$ species is already about $0.6 \mathrm{vol} \%$ at $1.51 \mathrm{\mu s}$ which is over three orders of magnitude higher than its neighbor penultimate species peak 


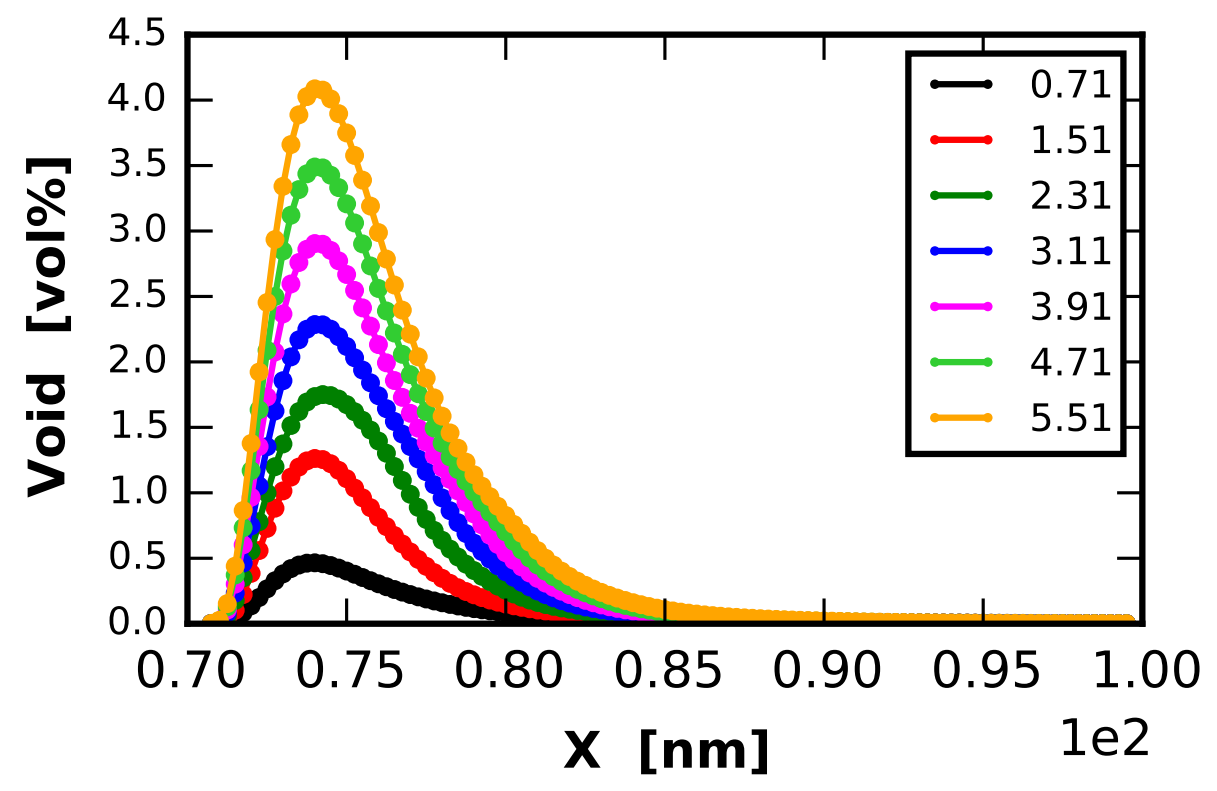

Fig. 3.1: Local tungsten void volume fraction, $\phi_{\mathrm{W}}(\boldsymbol{x}, t)$, for the $\mathrm{He}_{2_{40}}-V_{60}$ case at different times in units of $\mu \mathrm{s}$.

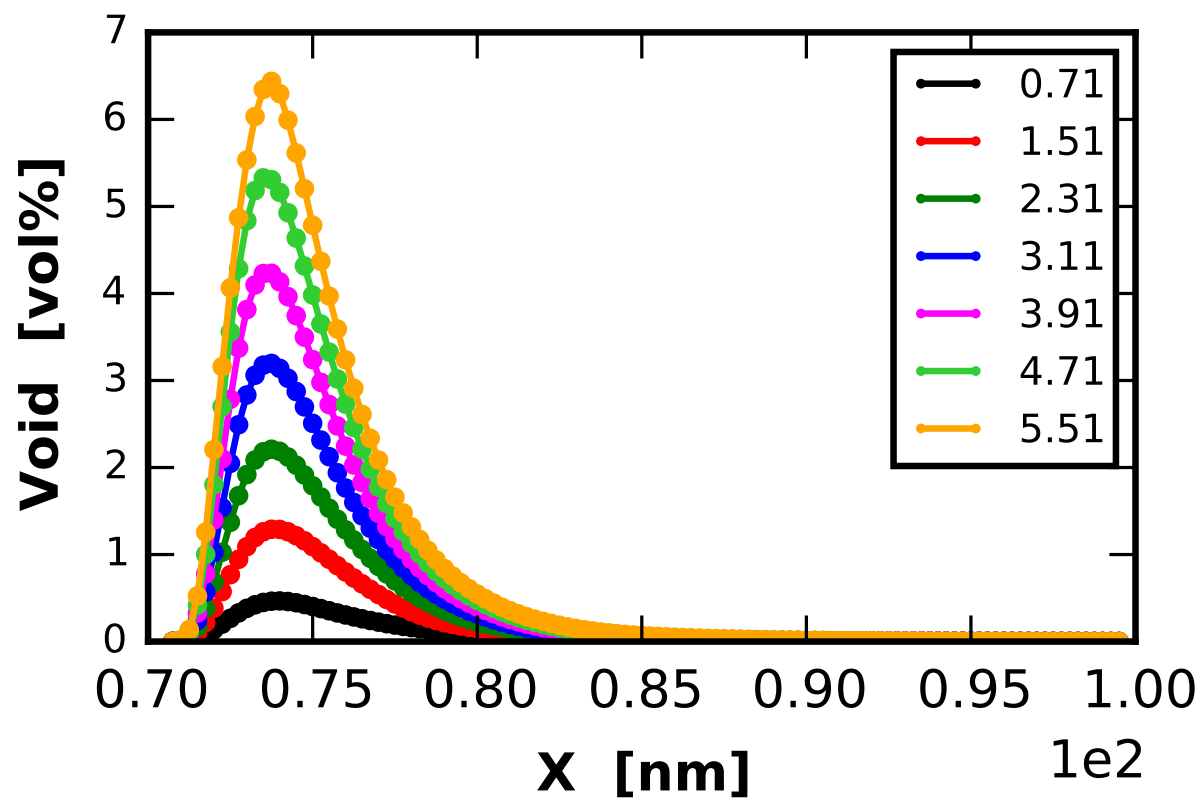

Fig. 3.2: Local tungsten void volume fraction, $\phi_{\mathrm{W}}(\boldsymbol{x}, t)$, for the $H_{1000}-V_{250}$ case at different times in units of $\mu \mathrm{s}$. 
volume fraction. In addition, there is sudden significant increase (eight orders of magnitude) in volume fraction peak for the terminal species (fig. 3.4, right plot) between times $0.71 \mu \mathrm{s}$ and $1.51 \mu \mathrm{s}$. Given the fact that peak values of volume fraction are above 1 vol \% for times greater than $1.51 \mu \mathrm{s}$ (fig. 3.3, right plot), it can be concluded that the simulation beyond this point is contaminated by this artificial growth of the terminal species. In addition, this is an nonphysical results since all other smaller size species should have nucleated before the terminal species which is not the case here (see plots in the Appendix A); all species between $\mathrm{He}_{199}-\mathrm{V}_{50}$ and $\mathrm{He}_{239}-\mathrm{V}_{60}$ do not nucleate before $\mathrm{He}_{240}-\mathrm{V}_{60}$ and this result is contradictory. Corresponding cumulative results for volume fraction of all species are also shown in the Appendix A demonstrating that the onset of nucleation of the terminal species prompts non-dilute defect interactions.
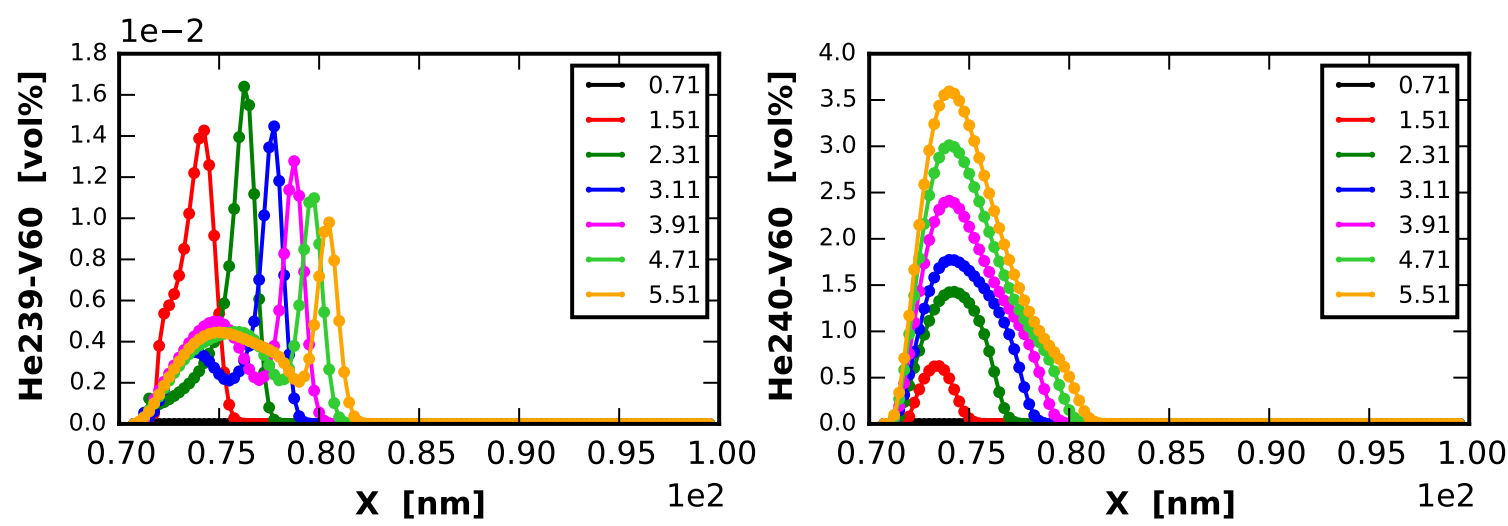

Fig. 3.3: Penultimate (left) and last (right) species volume fractions, $\phi_{a}(\boldsymbol{x}, t)$, for the $\mathrm{He}_{240}-V_{60}$ case at different times in units of $\mathrm{\mu s}$.
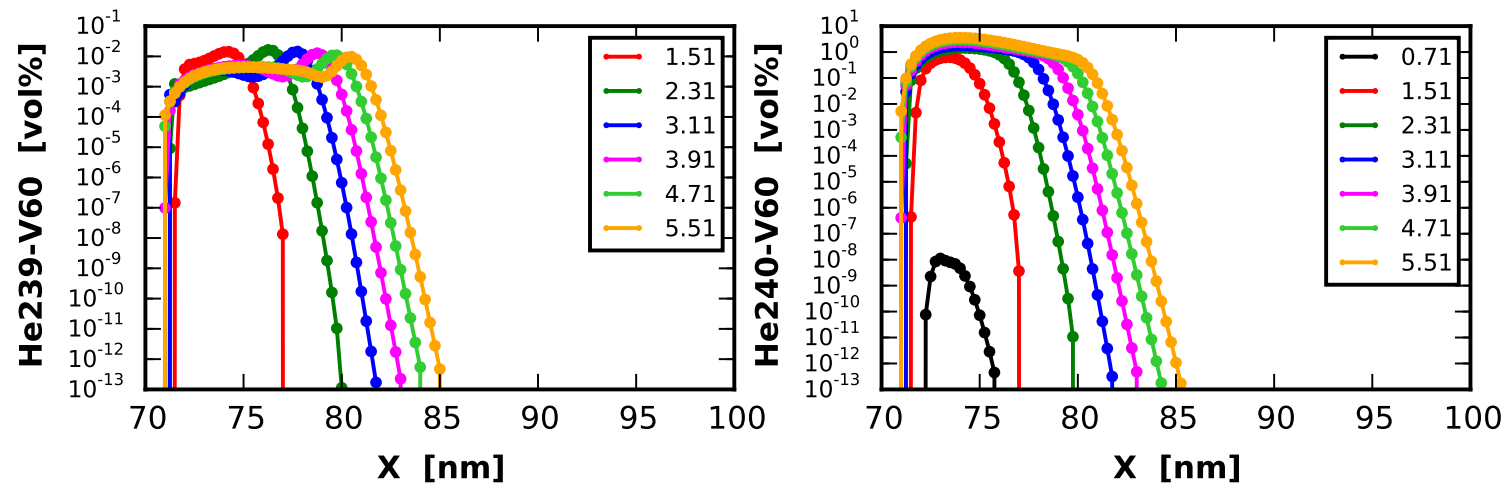

Fig. 3.4: Penultimate (left) and last (right) species volume fractions, $\phi_{a}(\boldsymbol{x}, t)$, for the $\mathrm{He}_{240}-V_{60}$ case at different times in units of $\mu \mathrm{s}$ (log-linear plots). 
Similar observations are made for the case with a larger list of species, case $\mathrm{He}_{1000^{-}}$ $\mathrm{V}_{250}$, except that a longer irradiation time can be reached in the simulation, say up to $5 \mu \mathrm{s}$, before the non-dilute regime emerges (figs. 3.5, 3.6). Note here that with a larger list of species, the difference in volume fractions between the penultimate species and the last species in the list is quantitative rather than qualitative. That is to say, a consistent result is obtained as far as the nucleation of the last species in the list is concerned. The largest-size species is the last to nucleate. A comparative view of all volume fractions for all species is showed in Appendix B.

Looking back at the first case (fig. 3.3), it is clear that as the simulation progresses in time, the initial Gaussian-like shape of the volume fraction develops a profile shoulder and eventually a double hump (fig. 3.3, left). This behavior is an artifact of the volume fraction growth of the last species beyond the dilute limit. The same behavior (less apparent) is observed in the second case (figs. 3.5, left). Therefore, by inspection, the departure from a Gaussian shape on the volume fraction profile coincides with non-dilute peak values of volume fraction and we are led to believe this could be used as an indication of the non-dilute transition point in simulation time. Furthermore, this transition point will vary depending on the size of the list of species used in the simulation and if the list is too short, the expected order of nucleation for the species may be affected. A consistent result should show the terminal species nucleation as happening last in time; unfortunately, this is not observed in any truncated form of cluster dynamics evolved long enough.
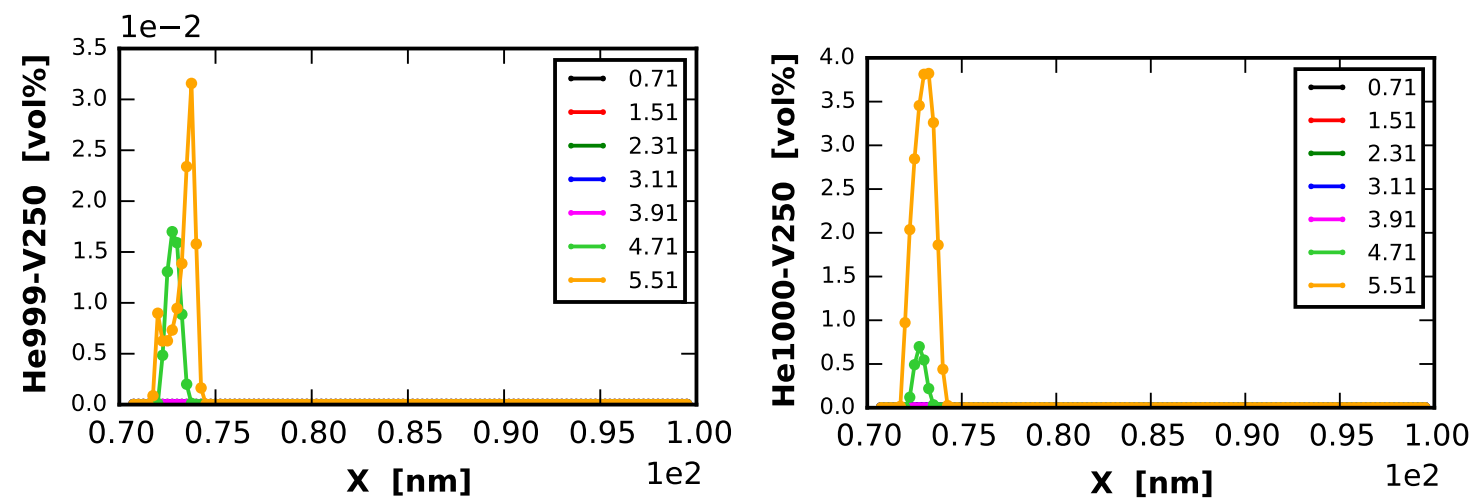

Fig. 3.5: Penultimate (left) and last (right) species volume fractions, $\phi_{a}(\boldsymbol{x}, t)$, for the $\mathrm{He}_{1000}-V_{250}$ case at different times in units of $\mathrm{\mu s}$.

It is instructive to compare the evolution of an intermediate helium-vacancy volume fraction for the two aforementioned cases. As a reminder, the case with the shorter list, $\mathrm{He}_{240}-\mathrm{V}_{60}$, has a total of $N=602$ species, and the long list case, $\mathrm{He}_{1000^{-}}$ $\mathrm{V}_{250}$, has a total of $N=1552$ species. There is substantial differences in the shape of the volume fraction between the two cases for the intermediate species $\mathrm{He}_{170}-\mathrm{V}_{40}$ 

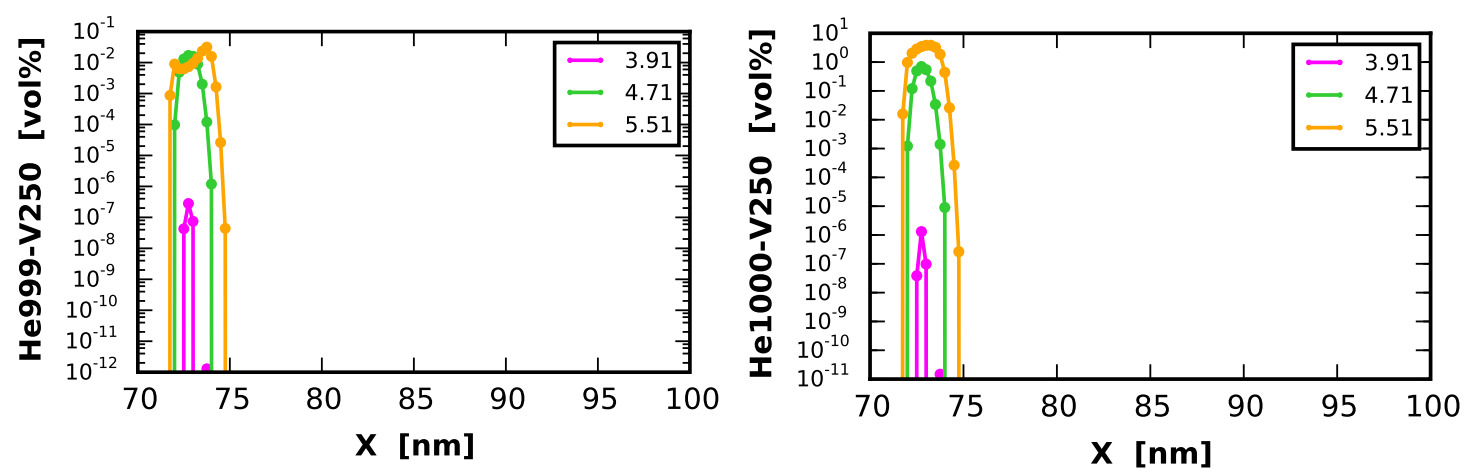

Fig. 3.6: Penultimate (left) and last (right) species volume fractions, $\phi_{a}(\boldsymbol{x}, t)$, for the $\mathrm{He}_{1000}-V_{250}$ case at different times in units of $\mathrm{us}$ (log-linear plots).

at longer times (figs. 3.7, 3.8) but this is a reflection of the non-dilute artifact of the terminal species as we have already mentioned. However, it is a relevant fact that the quantitative and qualitative results for irradiation times in the dilute regime are consistent between the two cases, that is, up to about time of $1 \mu \mathrm{s}$. While the individual peak values of volume fraction are low, around $10^{-2} \mathrm{vol} \%$, the sum of overlapping concentration profiles for all species lead to a void fraction representative of non-dilute regime (figs. 3.1 and 3.2) in about $1 \mu \mathrm{s}$ for both cases. This prompts the idea of introducing a physical void in the tungsten matrix for explicit representation of a bubble defect. Once proper boundary conditions of fluxes of all species are introduced on the bubble boundary, the simulation can proceed with this modified model assuming that the conditions of diffusion are dilute outside the bubble. This way the non-dilute condition is circumvented with the placement of the immersed bubble and associated boundary conditions (topic of the next section). The interesting aspect of this approach is that the simulation can be done with either one of the cases as long as the the introduction of the bubble is made as soon as non-dilute conditions emerge. Hence using the case with a lower number of species leads to a much lower-cost simulation.

In summary, these results have shown that the effect of the number of species on the evolution of the volume fractions is not significant up until the non-dilute effects emerge. After this point, the results are affected by artifacts and the results are nonphysical. In view of the localized evolution of the species volume fractions the peak value of the void volume fraction can be used to insert a bubble. This effectively generates a modified model that while using the dilute model outside the bubble domain is able to capture non-dilute behavior. This is further elaborated on the next section.

Oak Ridge National Laboratory Report ORNL/TM-2017/322 pp. 1-217 

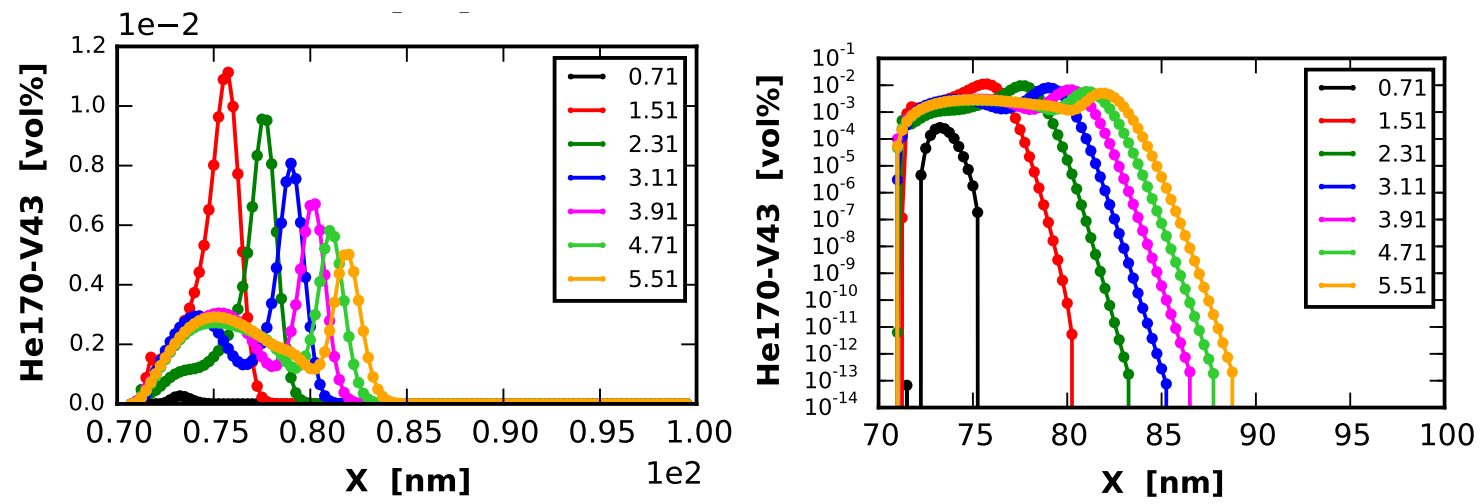

Fig. 3.7: Example of species helium-vacancy volume fraction, $\phi_{a}(\boldsymbol{x}, t)$, for the $\mathrm{He}_{240^{-}}$ $V_{60}$ case at different times in units of $\mathrm{us}$.
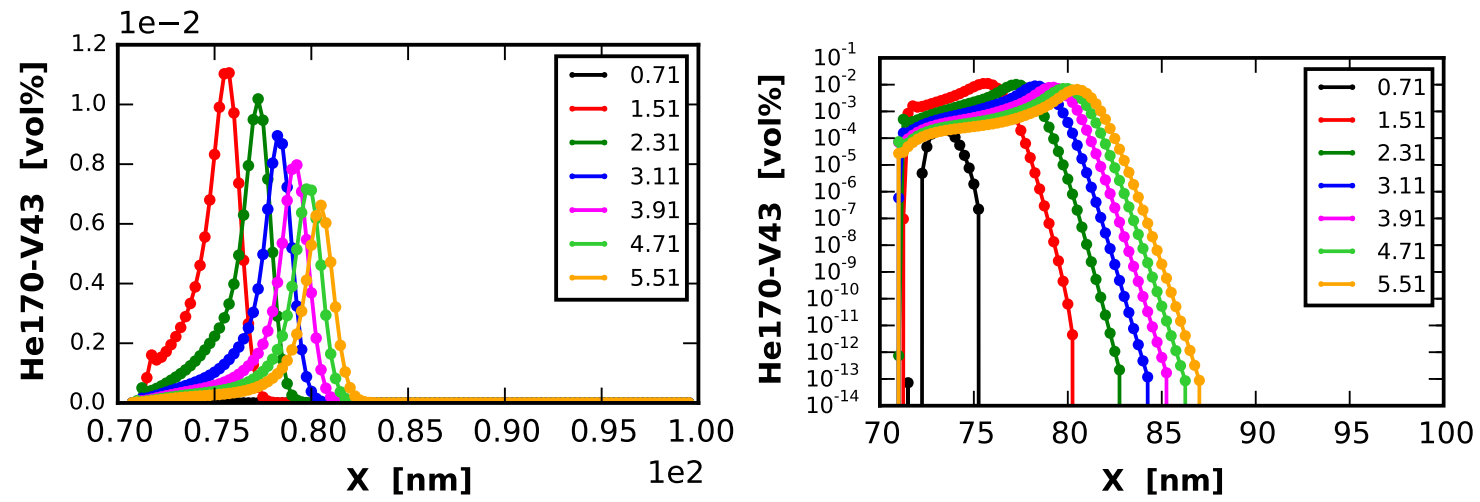

Fig. 3.8: Example of species helium-vacancy volume fraction, $\phi_{a}(\boldsymbol{x}, t)$, for the $\mathrm{He}_{1000^{-}}$ $V_{250}$ case at different times in units of $\mu \mathrm{s}$. 


\section{Modeling the non-dilute bubble regime}

From computational observations of the results of cluster dynamics simulations in the dilute limit presented previously (and in the Appendices), we demonstrated that the void volume fraction in tungsten develops a peak of about $1 \mathrm{vol} \%$ at around $1 \mu \mathrm{s}$ for both cases (different sizes of species list, figs. 3.1 and 3.2). Beyond this point in time, the results become adversely affected by the number of species used, and by the helium segregation in the tungsten. Hence a modification of the model to account for non-dilute defect evolution is desirable if not necessary. While a greater number of species reduces the adverse effect of a finite number of species, the calculations show that the void volume fraction is relatively insensitive to the number of species when a sufficiently large number of species is used (say, case $\mathrm{He}_{240}-\mathrm{V}_{60}$ ).

This prompts our proposed model for non-dilute cluster dynamics with bubble nucleation and growth. The void volume fraction is a simple field to monitor (fig. 3.1) and the approach here is extensible to 3-D. Denoting $t_{0}$ the time when a peak void volume fraction exceeds $1 \mathrm{vol} \%$, a domain denoted by $\Omega_{b}\left(t_{0}\right)$, and a corresponding barycenter point denoted as $\boldsymbol{x}_{b}$ can be found as the support of the void fraction function so that $\Phi_{\mathrm{W}}\left(\boldsymbol{x}, t_{0}\right) \gtrsim 1$, thus, $\Omega_{b}\left(t_{0}\right):=\left\{\boldsymbol{x} \mid \Phi_{\mathrm{W}}\left(\boldsymbol{x}, t_{0}\right) \gtrsim 1\right\}$ (fig. 4.1). The domain $\Omega_{b}\left(t_{0}\right)$ is created as an internal void by cutting it out from the original

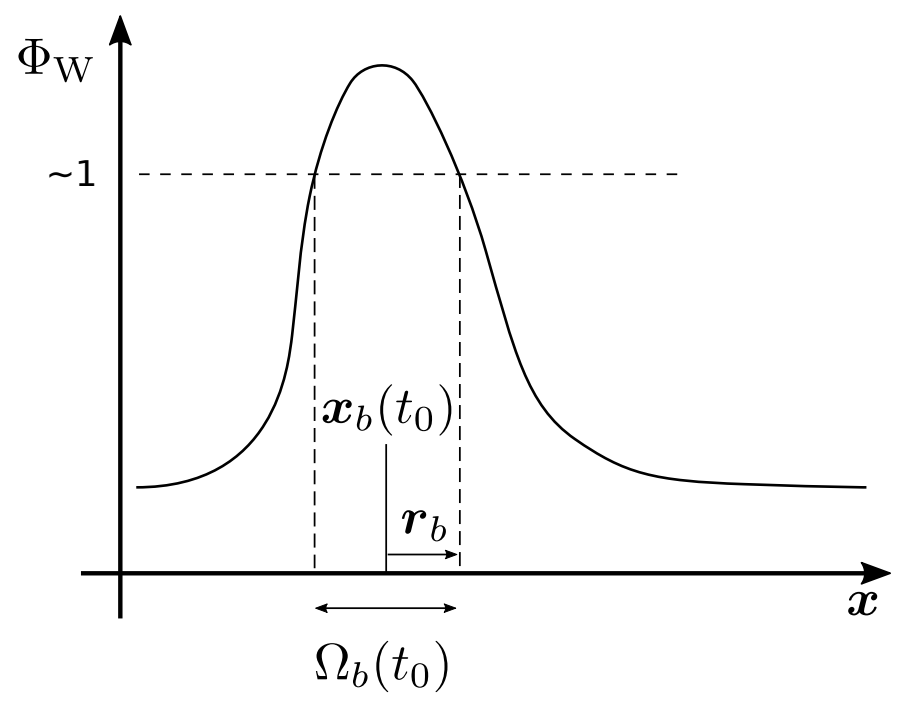

Fig. 4.1: Example of bubble domain at time $t_{0}$, with radius $\boldsymbol{r}\left(t_{0}\right)$, and center $\boldsymbol{x}_{b}\left(t_{0}\right)$.

continuous domain used in the cluster dynamics simulation; we refer to this domain as a bubble. The corresponding amount of He atoms at time $t_{0}$ in the bubble is then 
given by

$$
n_{\mathrm{He}}^{(b)}\left(t_{0}\right):=\sum_{1}^{M} \int_{\Omega_{b}\left(t_{0}\right)} n_{k, \mathrm{He}} c_{k, \mathrm{He}}\left(\boldsymbol{x}, t_{0}\right) d v,
$$

where $c_{k, \mathrm{He}}$ is the concentration of a He-containing species, $n_{k, H e}$ is the number of He atoms in the species, and $M$ is the total number of helium-containing species. A similar integration can be defined for the number of vacancies and interstitials in the bubble $\Omega_{b}\left(t_{0}\right)$. The bubble can be given a radius $\boldsymbol{r}_{b}\left(t_{0}\right)$ (fig. 4.1) and one of the purposes of the model is to define conditions under which this radius grows.

To this end, we exclude the bubble domain from the domain of all species concentration fields and introduce boundary conditions on the bubble boundary assuming the bubble center $\boldsymbol{x}_{b}$ to be stationary that is $\boldsymbol{x}_{b}\left(t_{0}\right)=$ const (fig. 4.2). Although this condition can be relaxed in a more realistic model, this is a useful simplifying assumption in the absence of deformation. It is important to comment on the fact

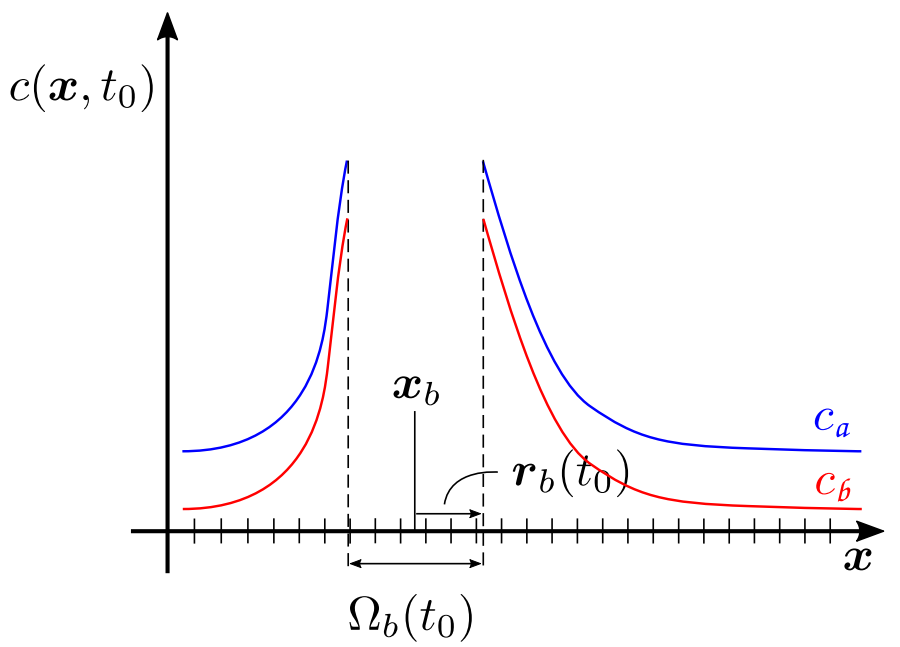

Fig. 4.2: Bubble domain insertion on all concentration fields at time $t_{0}$, with radius $\boldsymbol{r}\left(t_{0}\right)$, and center $\boldsymbol{x}_{b}\left(t_{0}\right)$.

that the concentration profiles over the bubble domain may depart significantly from a Gaussian shape, in particular for the smaller size species (e.g. fig. 4.3). The sections of all concentration profiles intercepted by the bubble contribute to the total amount of He accumulated into the bubble by computing $n_{\mathrm{He}}^{(b)}\left(t_{0}\right)$ at the initial time of formation of the bubble. Once the bubble is inserted, the growth of the bubble is a more intricate issue that depends on the bubble boundary flux of species.

The growth of $\Omega_{b}(t)$ for $t>t_{0}$ depends on the flux of species into and out of the bubble. Hence the normal component of the flux at the boundary of the bubble

$$
\boldsymbol{j}_{a}\left(\boldsymbol{x}_{s}, t\right) \cdot \boldsymbol{n}\left(\boldsymbol{x}_{s}, t\right) \quad \boldsymbol{x}_{s} \in \partial \Omega_{b}(t)
$$




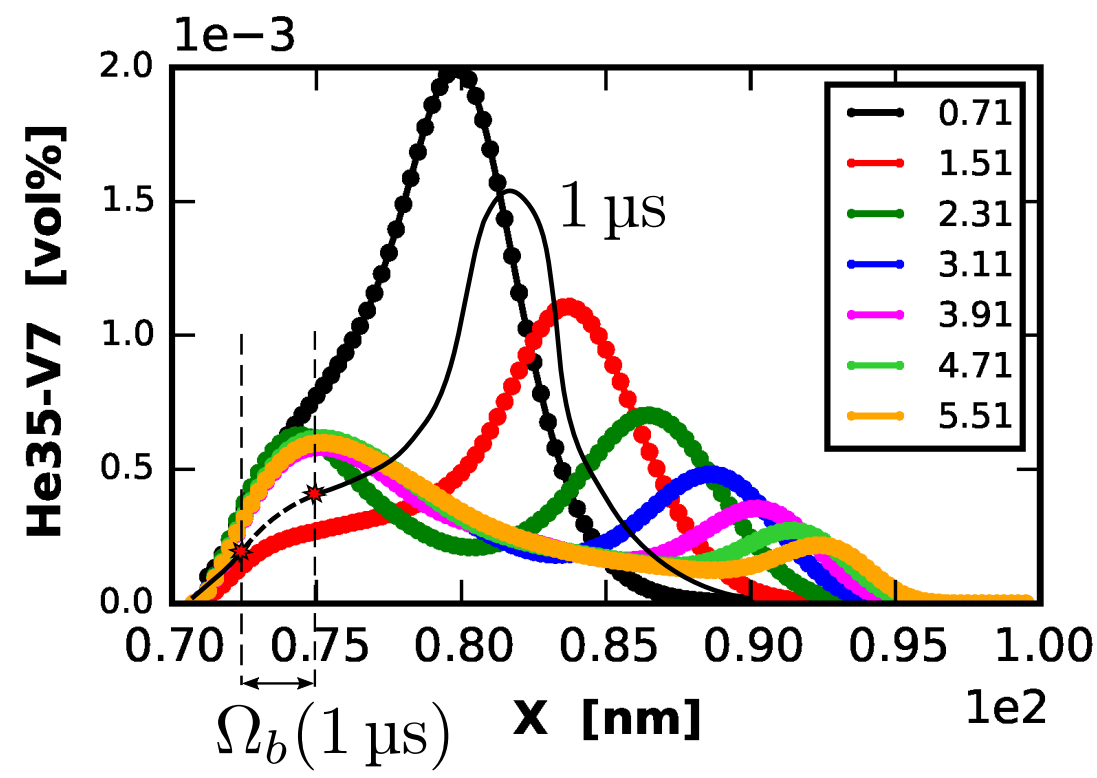

Fig. 4.3: Sketch of a bubble cut on a relatively small-size cluster at $t_{0}=1$ s showing the expected removed section of the concentration curve between red star points as a dotted curve. Case $\mathrm{He}_{240}-V_{60}$, Appendix A.

needs to be specified where $\boldsymbol{n}$ is the outward unit normal vector of the bubble surface and $\boldsymbol{x}_{s}$ denotes a point on the bubble surface. The bubble growth source term due to the flow of He alone is given by

$$
\dot{S}_{\mathrm{He}}^{(b)}(t):=\sum_{k=1}^{M} n_{k, \mathrm{He}} \int_{\partial \Omega_{b}(t)} \boldsymbol{j}_{k, \mathrm{He}} \cdot(-\boldsymbol{n}) d a ;
$$

note the negative sign is a reflection of the orientation of the normal vector pointing into the surrounding solid matrix. A similar expression is expected for the contributions of vacancies and interstitials to the growth of the bubble. The total number of He atoms at any time is given by adding (4.1) to 4.2

$$
n_{\mathrm{He}}^{(b)}(t)=n_{\mathrm{He}}^{(b)}\left(t_{0}\right)+\dot{S}_{\mathrm{He}}^{(b)}(t) .
$$

A simple argument for bubble volume expansion or contraction based on $\dot{S}_{\mathrm{He}}^{(b)}$ leads to

$$
\mathrm{d}_{t} r_{b}=\frac{\Gamma_{\mathrm{He}} \dot{S}_{\mathrm{He}}^{(b)}(t)}{4 \pi r_{b}^{2}}
$$

which provides the rate of change of the radius of the bubble as a function of a coefficient of bubble expansion, $\Gamma_{\text {He }}$, to be determined from either other level of theory 
or experimental data if possible. The complete bubble growth source term would include sources of vacancies and interstitials, hence, $\dot{S}_{\mathrm{V}}^{(b)}$ and $\dot{S}_{\mathrm{I}}^{(b)}$. The kinematics of the bubble for a fixed barycenter $\boldsymbol{x}_{b}$ allows for the normal motion of the bubble boundary as

$$
\boldsymbol{x}_{s}(t)-\boldsymbol{x}_{b}=r_{b}(t) \boldsymbol{n}
$$

and from time differentiation

$$
\mathrm{d}_{t} \boldsymbol{x}_{s}(t)=\mathrm{d}_{t} r_{b} \boldsymbol{n}
$$

giving

$$
\mathrm{d}_{t} \boldsymbol{x}_{s}(t)=\frac{\Gamma_{\mathrm{He}} \dot{S}_{\mathrm{He}}^{(b)}(t)}{4 \pi} \frac{1}{r_{b}^{2}} \boldsymbol{n},
$$

as the kinematic equation of the stationary (spherical) bubble surface. Note that the $r_{b}^{-2}$ term is the Gaussian curvature of a sphere and the equation can be expressed in a more general form

$$
\mathrm{d}_{t} \boldsymbol{x}_{s}(t)=\frac{\Gamma_{\mathrm{He}} \dot{S}_{\mathrm{He}}^{(b)}(t)}{4 \pi} \mathscr{K}\left(\boldsymbol{x}_{s}, t\right) \boldsymbol{n},
$$

which could be postulated for other generic shapes with a surface dependent curvature $\mathscr{K}\left(\boldsymbol{x}_{s}, t\right)$; this is particularly useful in the case of bubble coalescence described later. Therefore, integration in time of the kinematic equation when coupled to the reaction/diffusion equations of cluster dynamics in the dilute limit allows for growth of the bubble and further evolution of the governing equations which should apply to regions of the domain that are expected to satisfy the dilute regime.

We turn attention now to the calculation of the bubble growth source $\dot{S}_{\mathrm{He}}^{(b)}$ which requires an expression for the normal fluxes $\boldsymbol{j}_{k, \mathrm{He}} \cdot(-\boldsymbol{n})$. Here we postulate that all species not containing He atoms do not contribute to the growth of the bubble, hence

$$
\begin{aligned}
& \boldsymbol{j}_{V_{j}}\left(\boldsymbol{x}_{s}, t\right) \cdot \boldsymbol{n}\left(\boldsymbol{x}_{s}, t\right)=0 \\
& \boldsymbol{j}_{I_{k}}\left(\boldsymbol{x}_{s}, t\right) \cdot \boldsymbol{n}\left(\boldsymbol{x}_{s}, t\right)=0
\end{aligned}
$$

$\forall \quad t \geq t_{0}$ and $\boldsymbol{x}_{s} \in \partial \Omega_{b}(t)$. For the He-containing species we define an interfacial equilibrium concentration as a function of the number of He atoms and vacancies in the bubble

$$
c_{k, \mathrm{He}}^{*}\left(n_{\mathrm{He}}^{(b)}(t), n_{\mathrm{V}}^{(b)}(t)\right) .
$$

Next we define the diffusive normal flux on the bubble boundary using the departure of the concentration at the interface from the equilibrium value

$$
\left.\boldsymbol{j}_{k, \mathrm{He}}\left(\boldsymbol{x}_{s}, t\right) \cdot\left(-\boldsymbol{n}\left(\boldsymbol{x}_{s}, t\right)\right)=h_{k, \mathrm{He}}\left(c_{k, \mathrm{He}}\left(\boldsymbol{x}_{s}, t\right)-c_{k, \mathrm{He}}^{*}\right)\right)
$$


where $h_{k, \mathrm{He}}$ is the species interfacial transfer coefficient. At the moment of bubble nucleation, the right side of (4.7) will be positive, hence this will sustain the growth of the bubble until the interfacial concentration reaches the equilibrium level. As a matter of numerical testing of this boundary condition, the equilibrium value can be taken as a fraction of the average interfacial concentration at the moment of nucleation, say

$$
c_{k, \mathrm{He}}^{*}=\alpha^{-1} \int_{\partial \Omega_{b}\left(t_{0}\right)} c_{k, \mathrm{He}}\left(\boldsymbol{x}_{s}, t_{0}\right) d a,
$$

and held constant for the remaining of the simulation. This allows for the bubble to grow initially and either come to an equilibrium or allow for re-solvation later in time. Both the equilibrium interfacial concentration and interfacial transfer coefficients are parameters needed from either experimental work or atomistic simulations.

This brings us to a comment on bubble coalescence. In the event of two bubbles nucleate and begin to grow, they could eventually coalesce. In this case, the generic kinematics proposed, (4.4), can be used as a preliminary model for evolving the merged surface of two bubbles since the curvature is now a function of the shape of the surface and time as well (fig. 5.2).

\section{$5 \quad$ Bubble font-tracking}

The front-tracking method ( $\mathrm{Hu}$ et al., 2015) can be effectively used to evolve the shape of bubbles as described earlier (sec. 4) as a well-defined kinematic problem. Here follows a brief outline of the implementation needed to modify the Xolotl code to perform simulations with bubble nucleation, growth and drift. The front-tracking method can be used with the finite-difference discretization in Xolotl without modifying the existing mesh. The approach amounts to overlaying a surface mesh (or curve mesh in 2-D, or interval end points in 1-D) on the underlying Xolotl mesh and performing the discretization on a cut-cell mesh version of the original mesh (fig. 5.1). The fluxes on the boundary of the bubble need to be computed in the Xolotl code and either set to zero or to the interfacial normal diffusive flux (sec. 4). The evolution of the bubble using the front-tracking method uses directly the kinematic evolution equation (4.3) or (4.4) on marker points on the bubble surface (fig. 5.1). The interfacial diffusive conditions (4.5) and 4.7) must be implemented on the Xolotl code side when solving for the concentration fields.

Coalescence of bubbles (fig. 5.2) can be accommodated by employing the kinematic condition based on a variable local curvature (4.4) in conjunction with heuristic methods for geometry-based front untangling (Hu et al., 2015).

In summary, the front-tracking of He bubbles is a tractable computational problem 


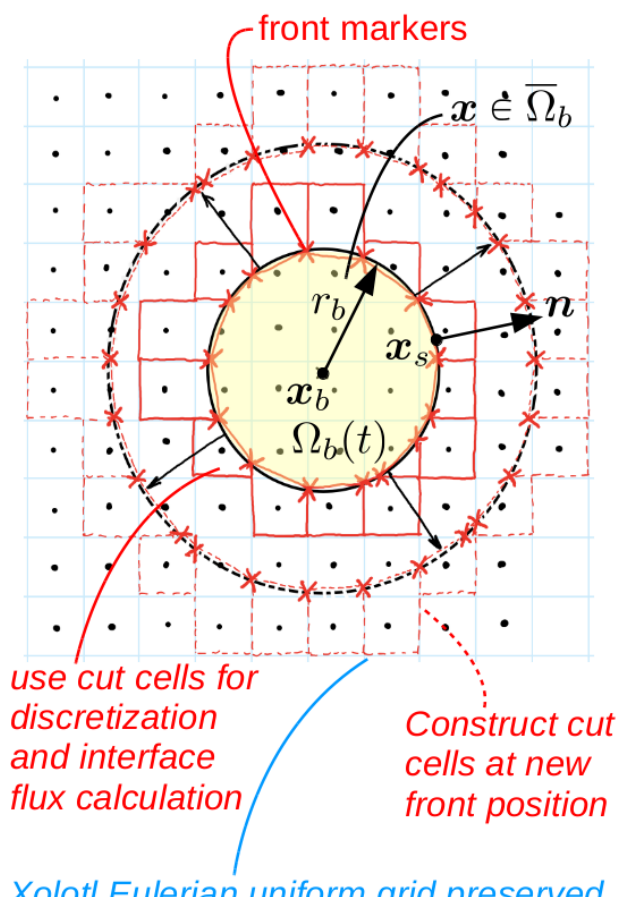

Xolotl Eulerian uniform grid preserved

Fig. 5.1: 2-D sketch of front-tracking evolution of bubble after nucleation.

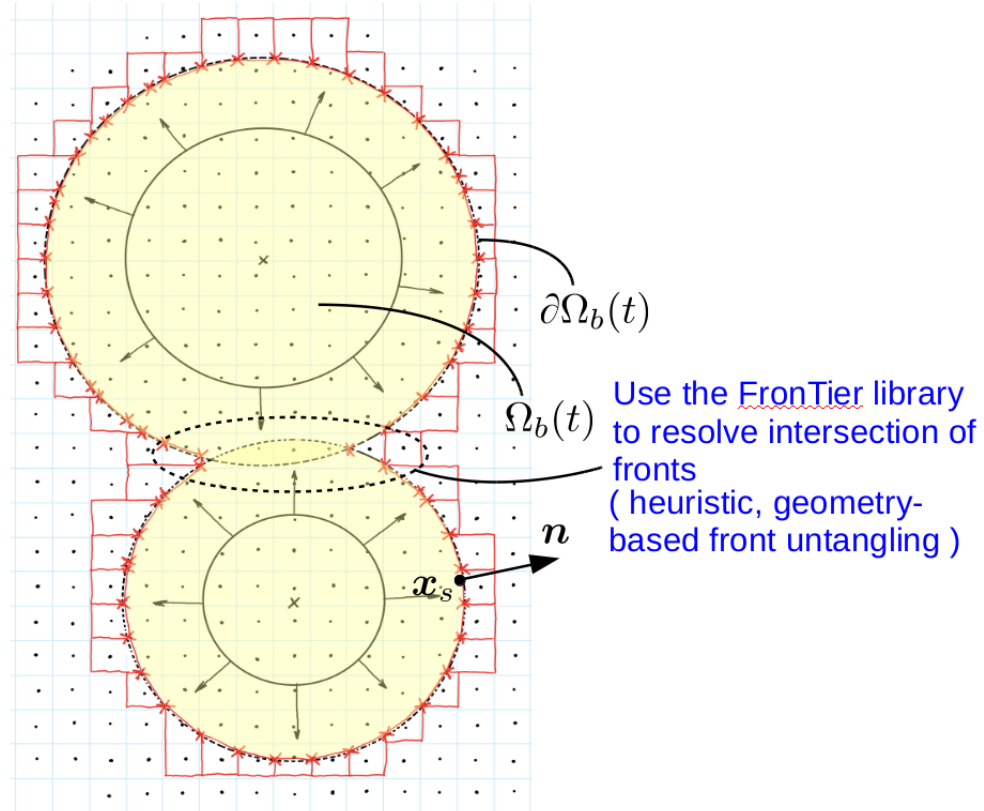

Fig. 5.2: 2-D sketch of front-tracking coalescence of bubbles.

Oak Ridge National Laboratory Report ORNL/TM-2017/322 pp. 1-217 
for cases where a void fraction peak can be identified. Nevertheless, as with any moving boundary problem, (4.4)-(4.7), there are challenges in practical implementations in Xolotl. Given the numerical experiments performed in this work to define the kinematics of bubble nucleation growth and coalescence, the approach presented attempts to bridge the modeling gap and complexity of solution method implementation. 
A Appendix: Case $\mathbf{H e}_{240}-\mathrm{V}_{60}$

A.1 Volume fraction plots (linear-linear) 
Transient: [0.71, 1.51, 2.31, 3.11, 3.91, 4.71, 5.51] [us]
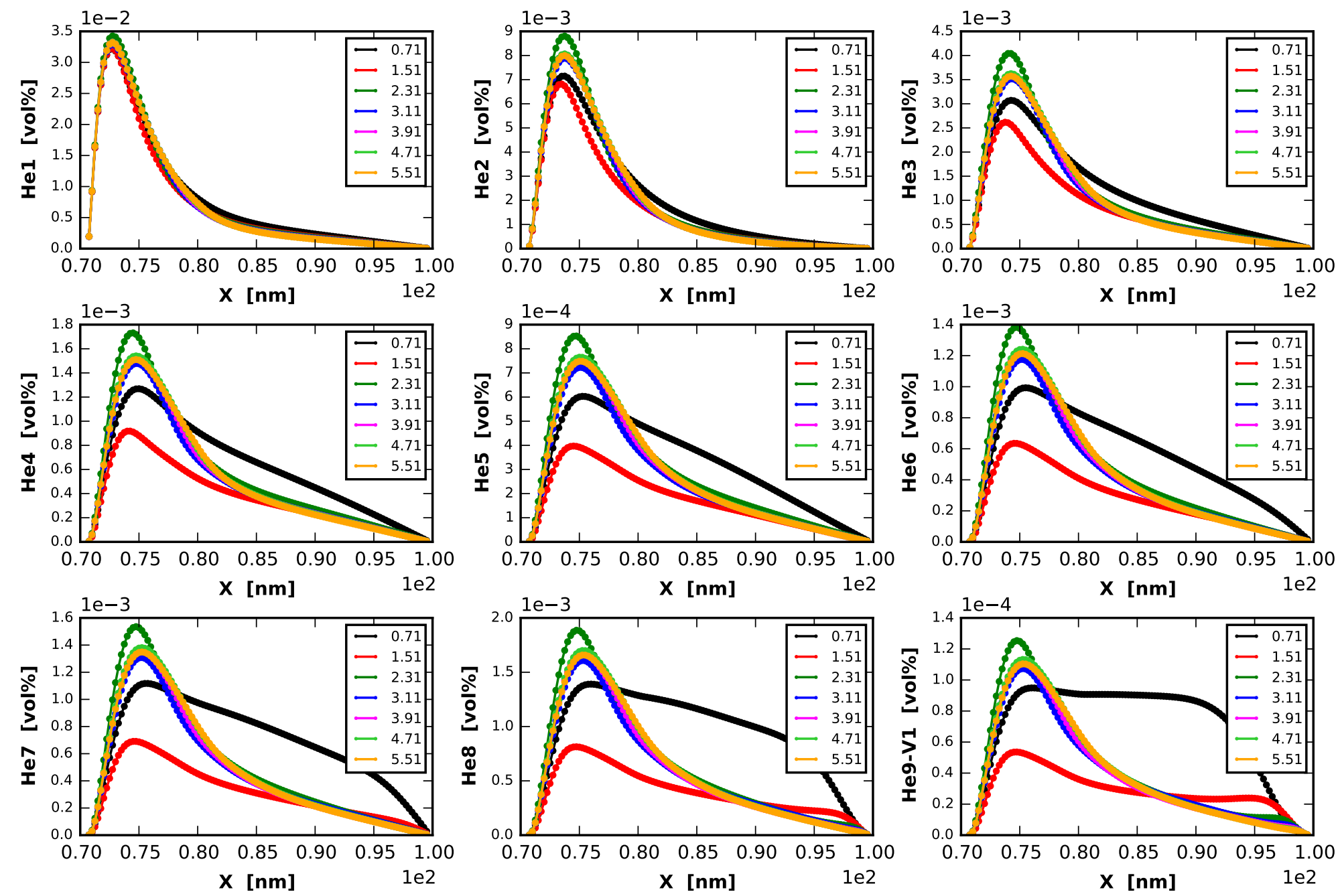
Transient: $[0.71,1.51,2.31,3.11,3.91,4.71,5.51]$ [us]
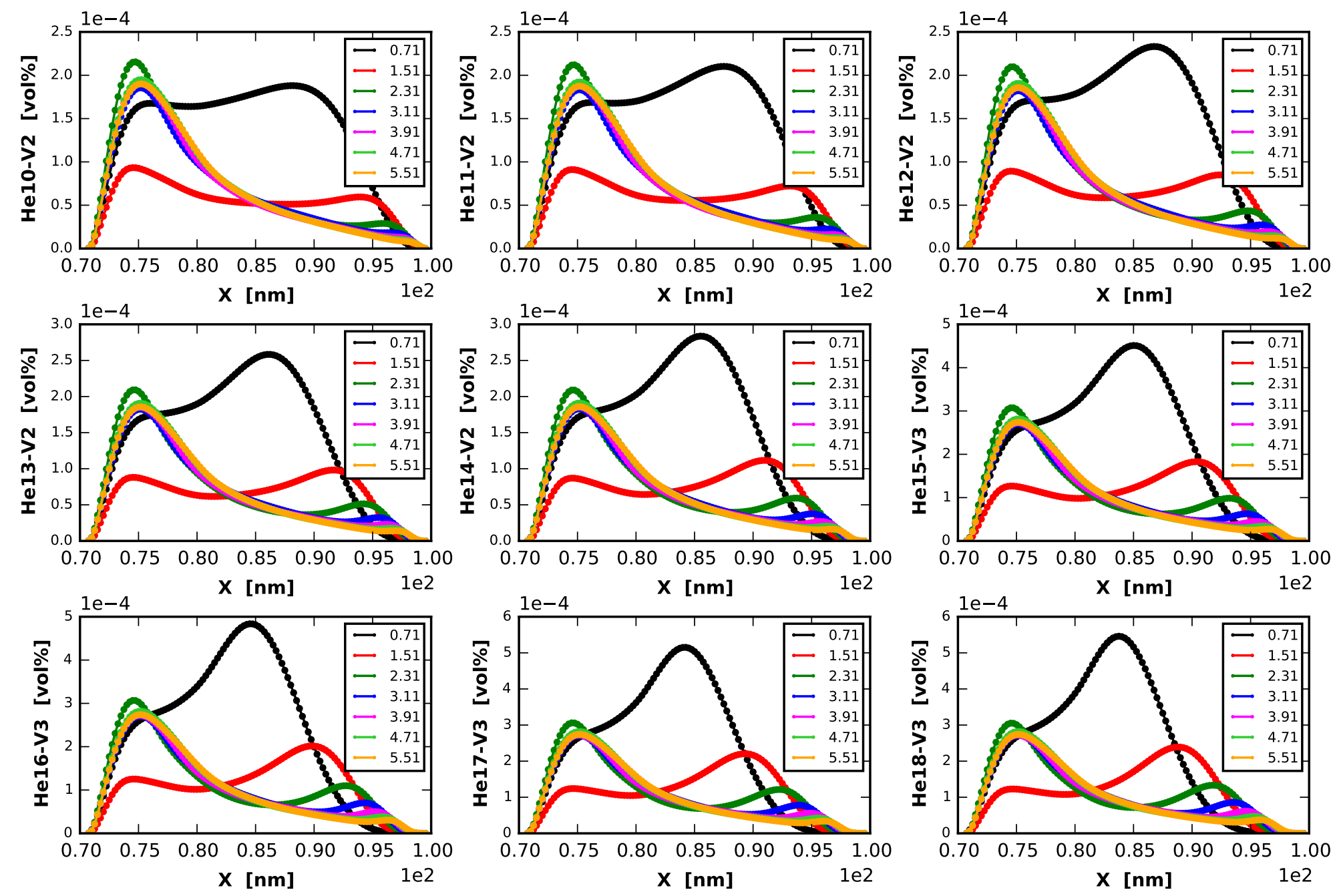
Transient: [0.71, 1.51, 2.31, 3.11, 3.91, 4.71, 5.51] [us]
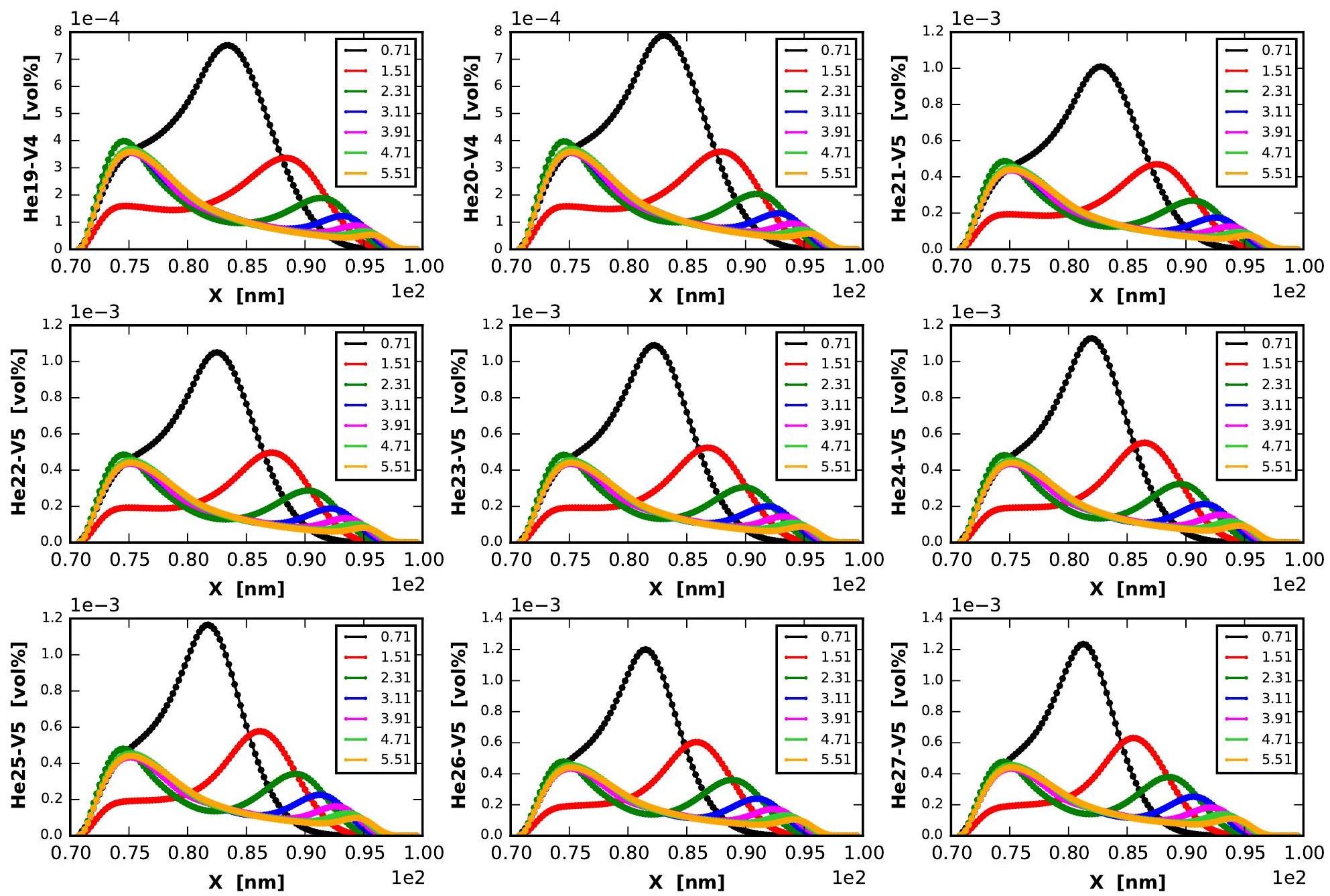
Transient: $[0.71,1.51,2.31,3.11,3.91,4.71,5.51]$ [us]
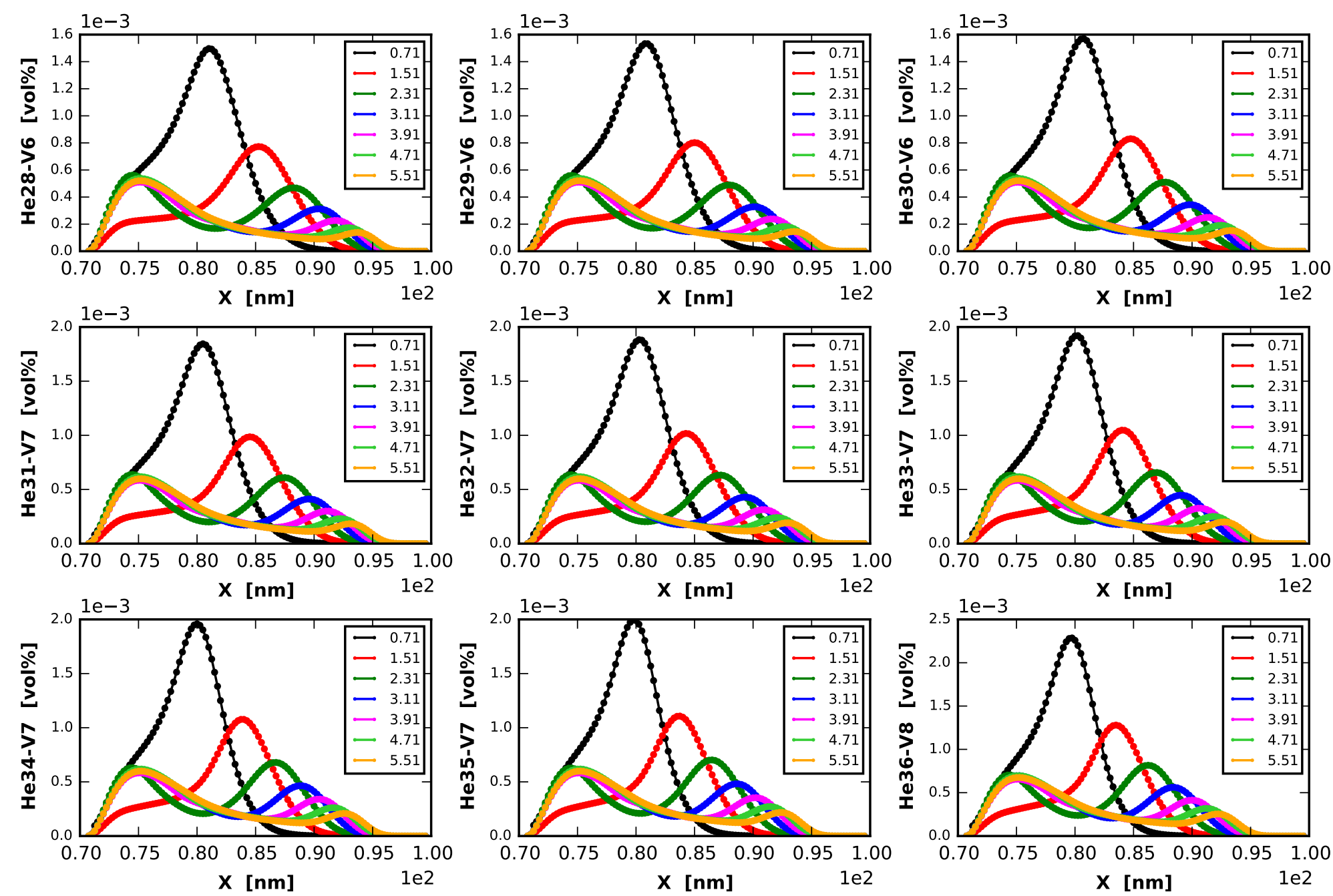
Transient: $[0.71,1.51,2.31,3.11,3.91,4.71,5.51]$ [us]
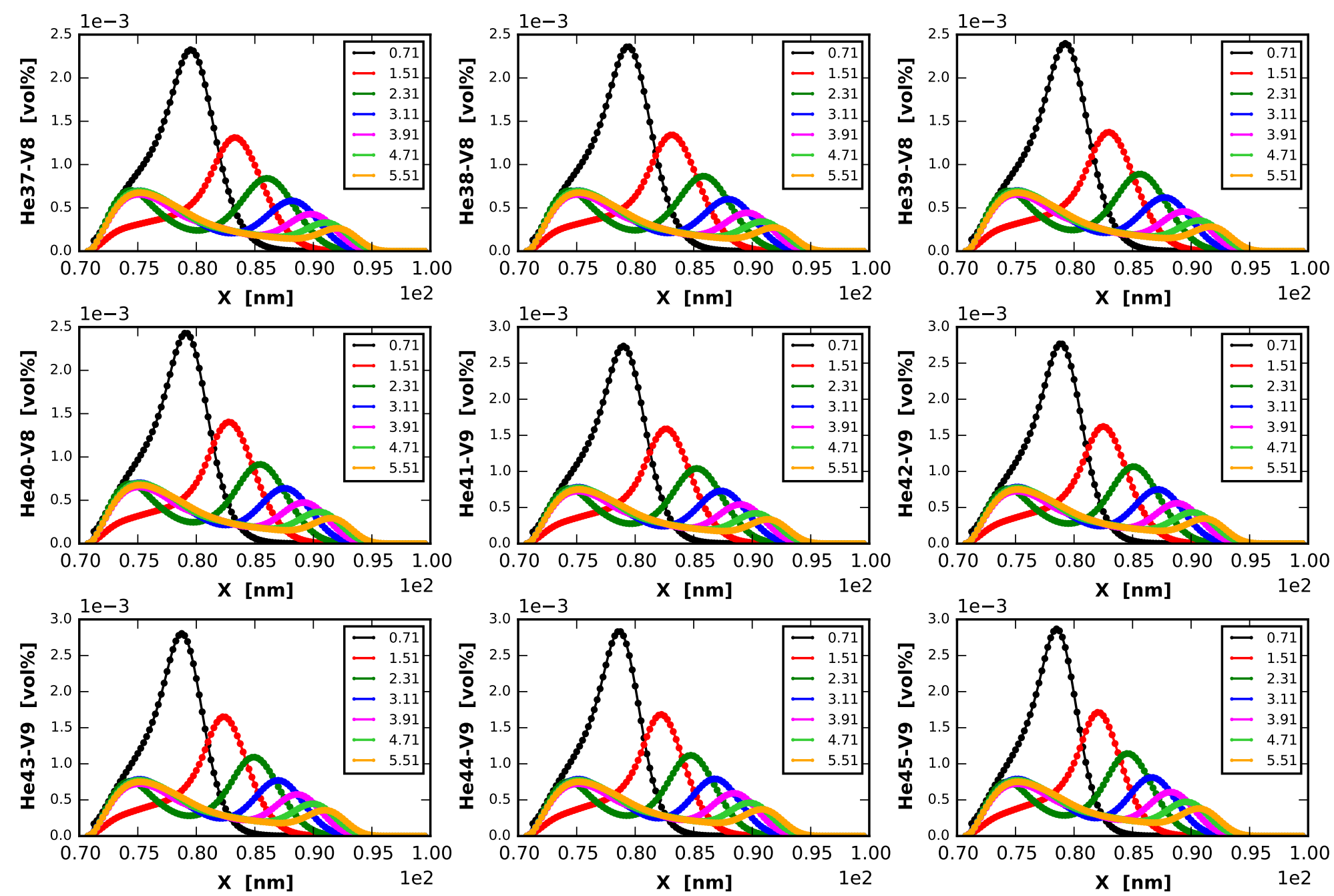


\section{Transient: [0.71, 1.51, 2.31, 3.11, 3.91, 4.71, 5.51] [us]}
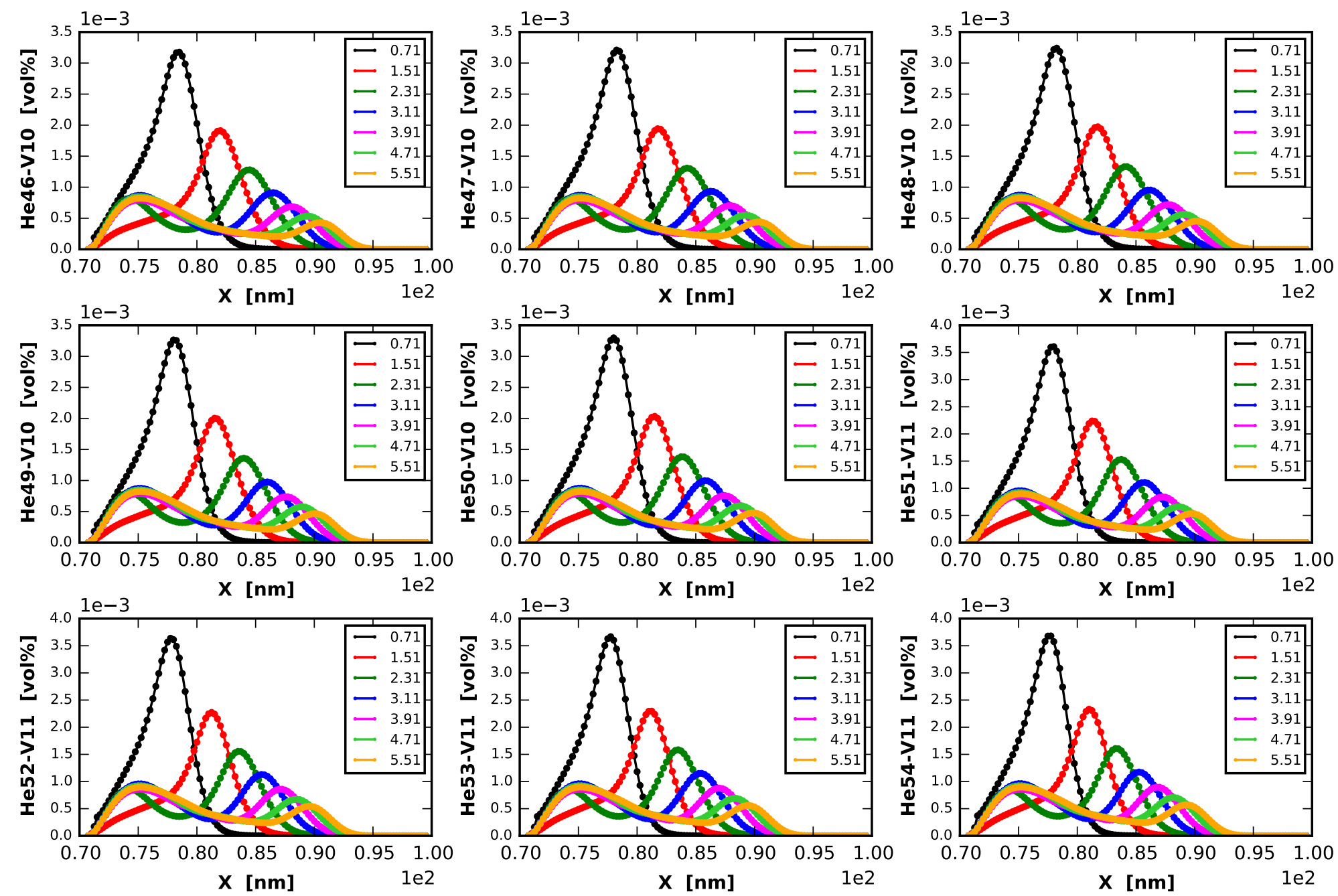

Transient: $[0.71,1.51,2.31,3.11,3.91,4.71,5.51]$ [us]
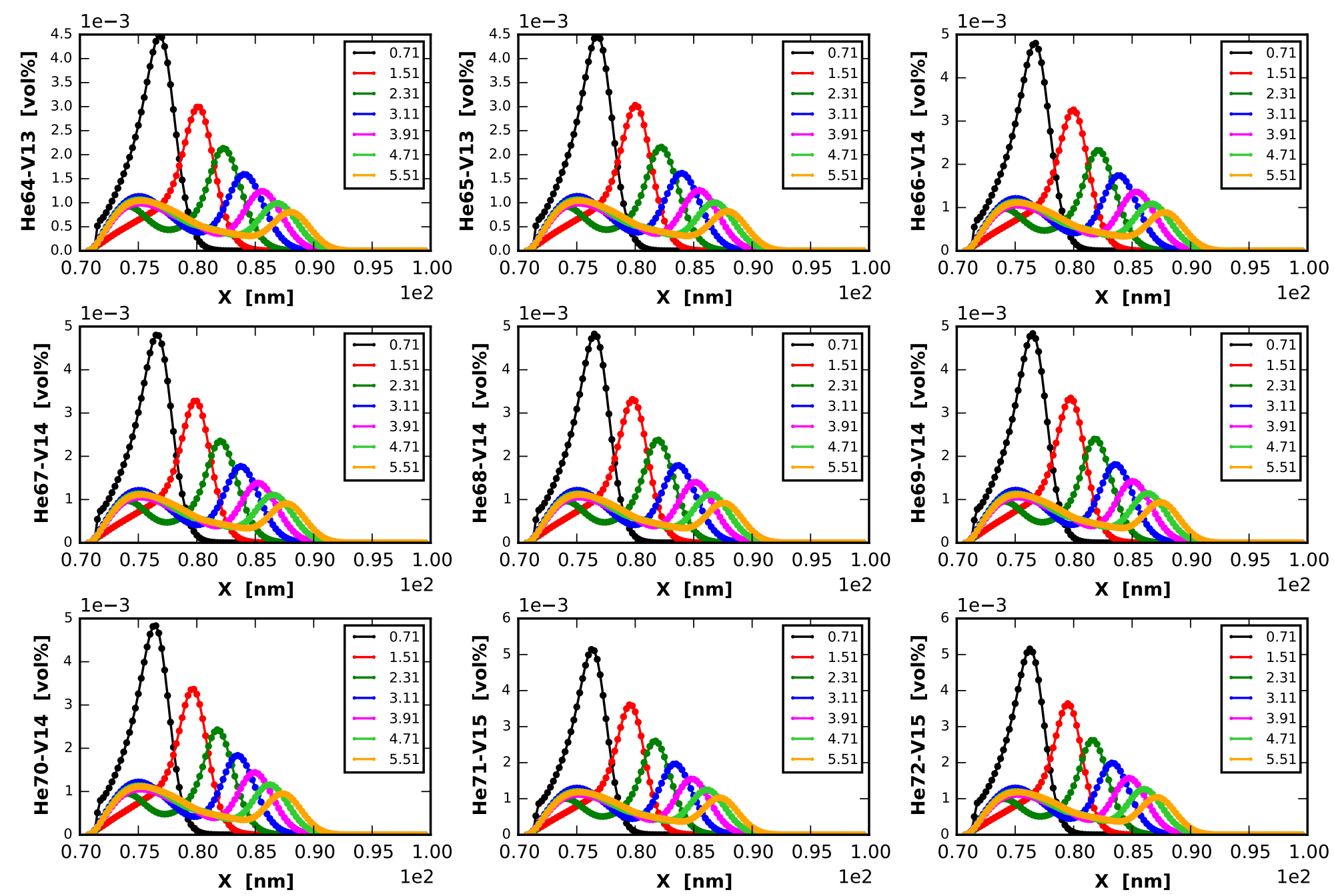
Transient: $[0.71,1.51,2.31,3.11,3.91,4.71,5.51]$ [us]
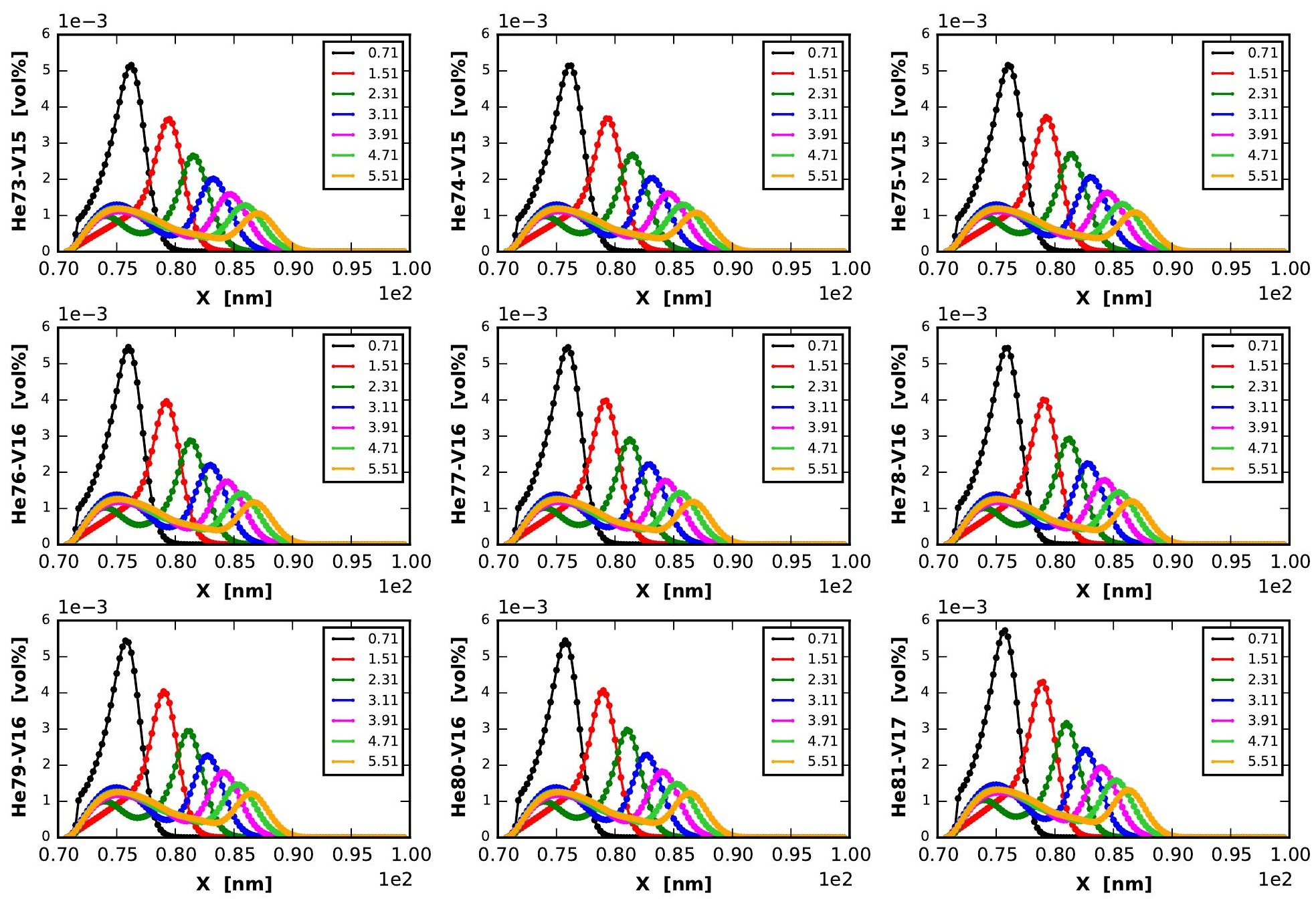
Transient: $[0.71,1.51,2.31,3.11,3.91,4.71,5.51]$ [us]
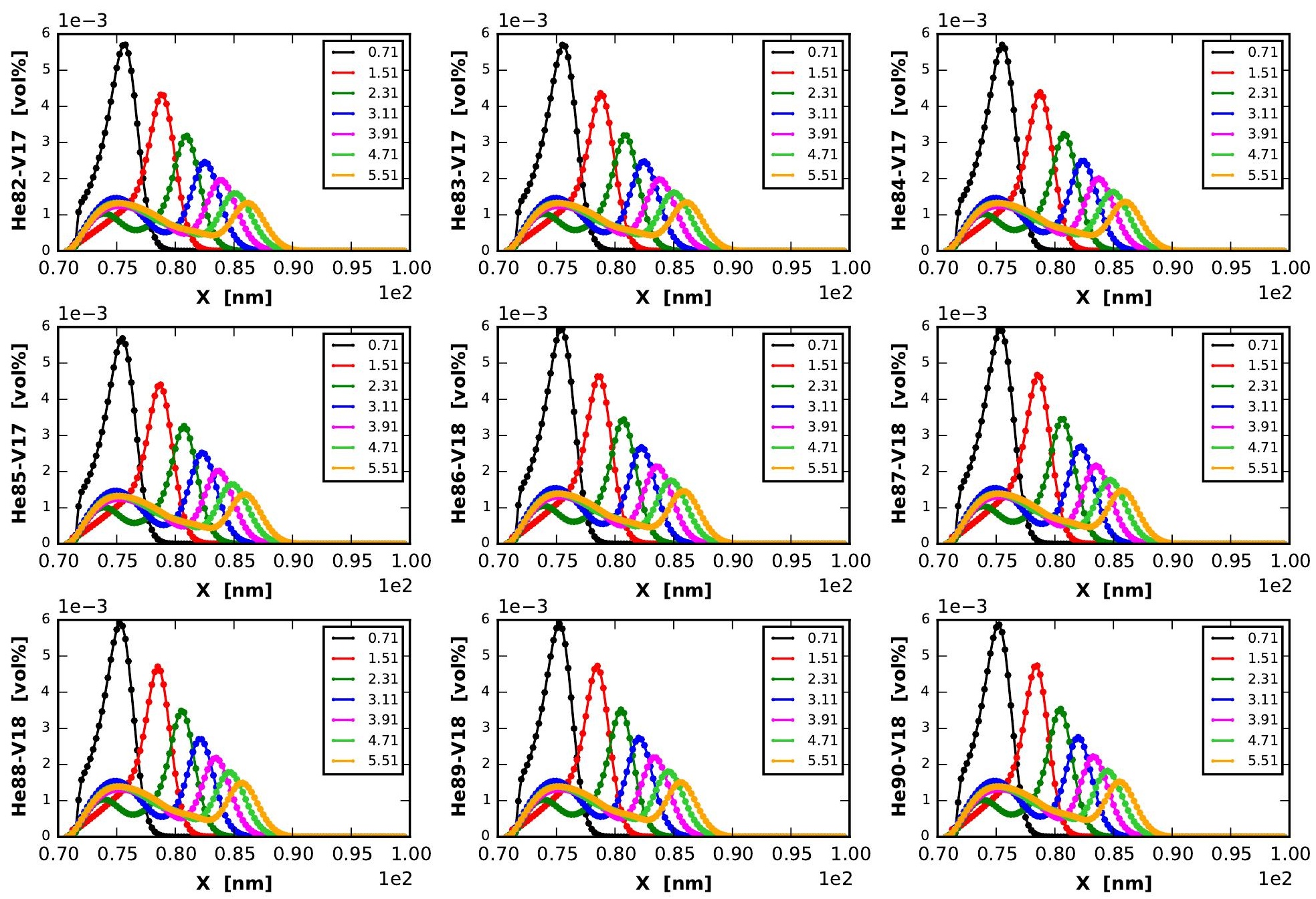
Transient: $[0.71,1.51,2.31,3.11,3.91,4.71,5.51]$ [us]
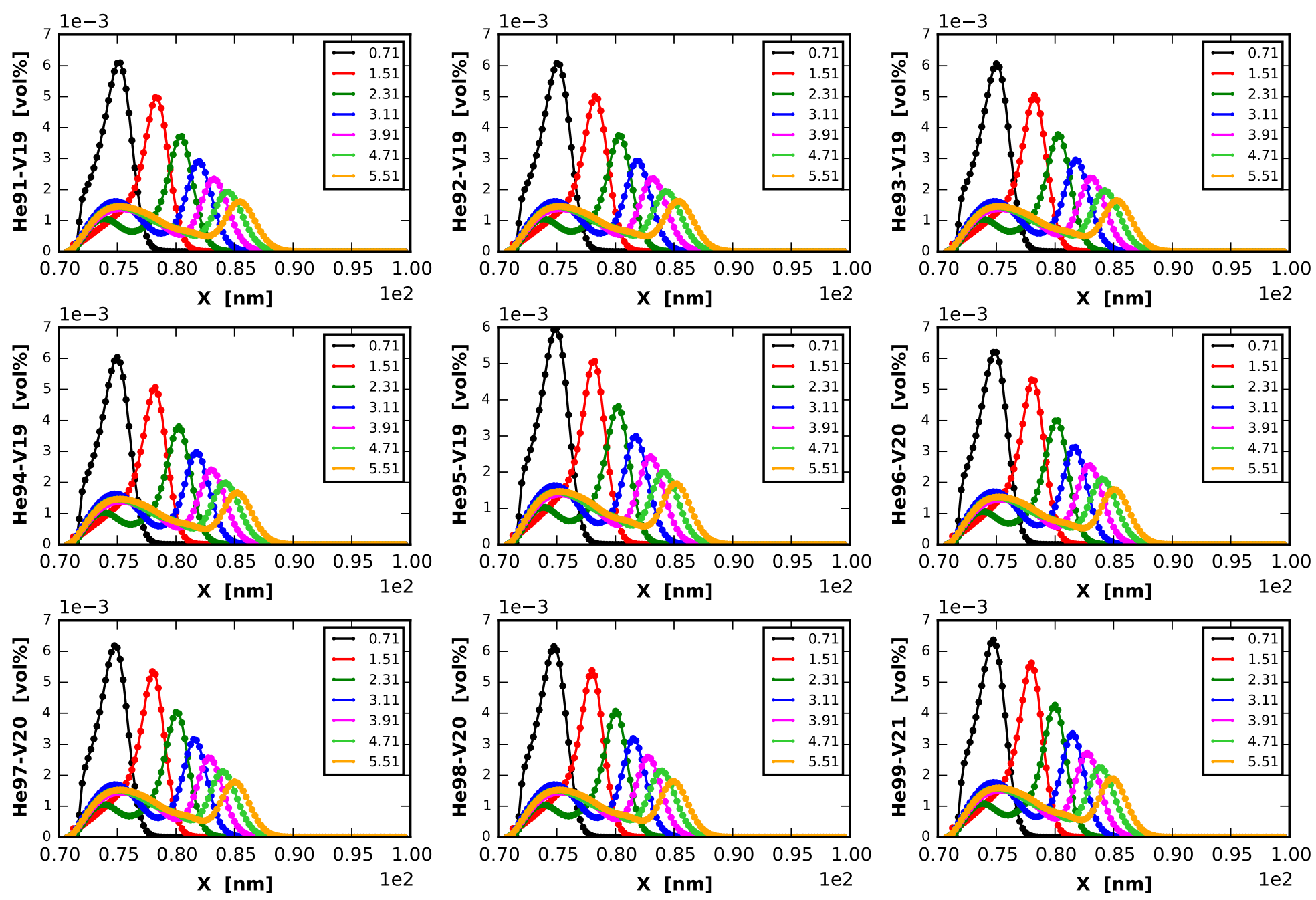
Transient: $[0.71,1.51,2.31,3.11,3.91,4.71,5.51]$ [us]
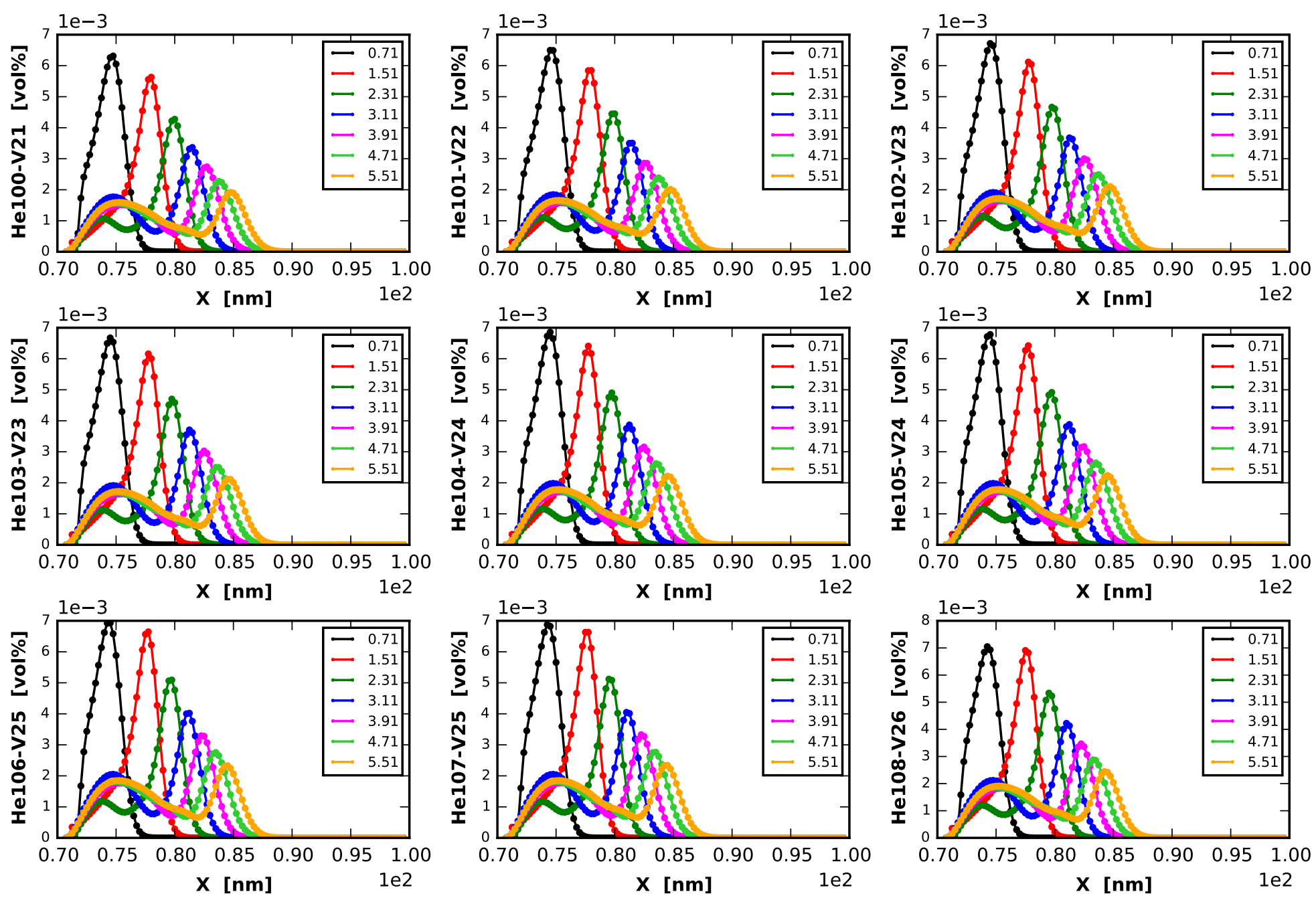
Transient: $[0.71,1.51,2.31,3.11,3.91,4.71,5.51]$ [us]
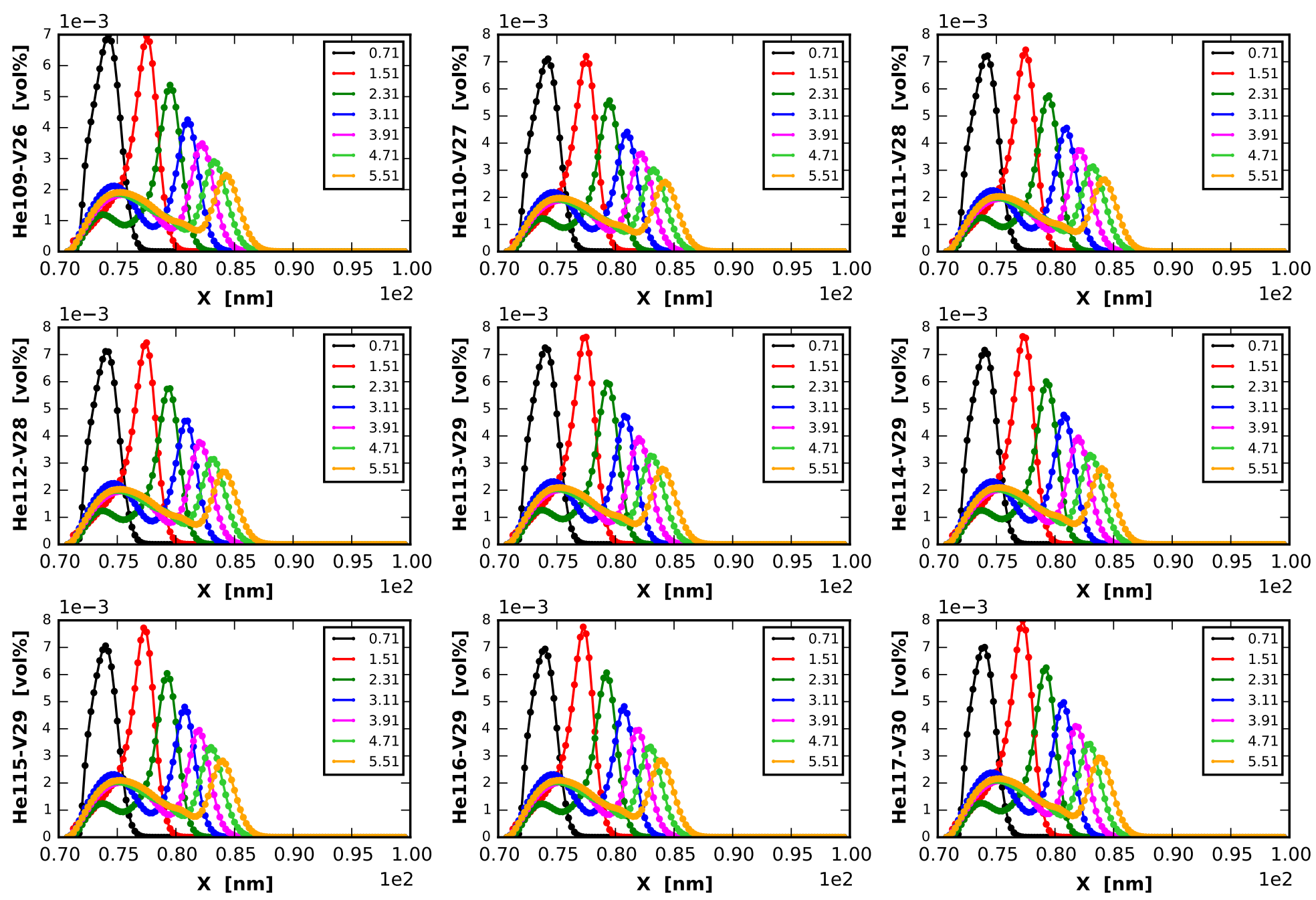
Transient: $[0.71,1.51,2.31,3.11,3.91,4.71,5.51]$ [us]
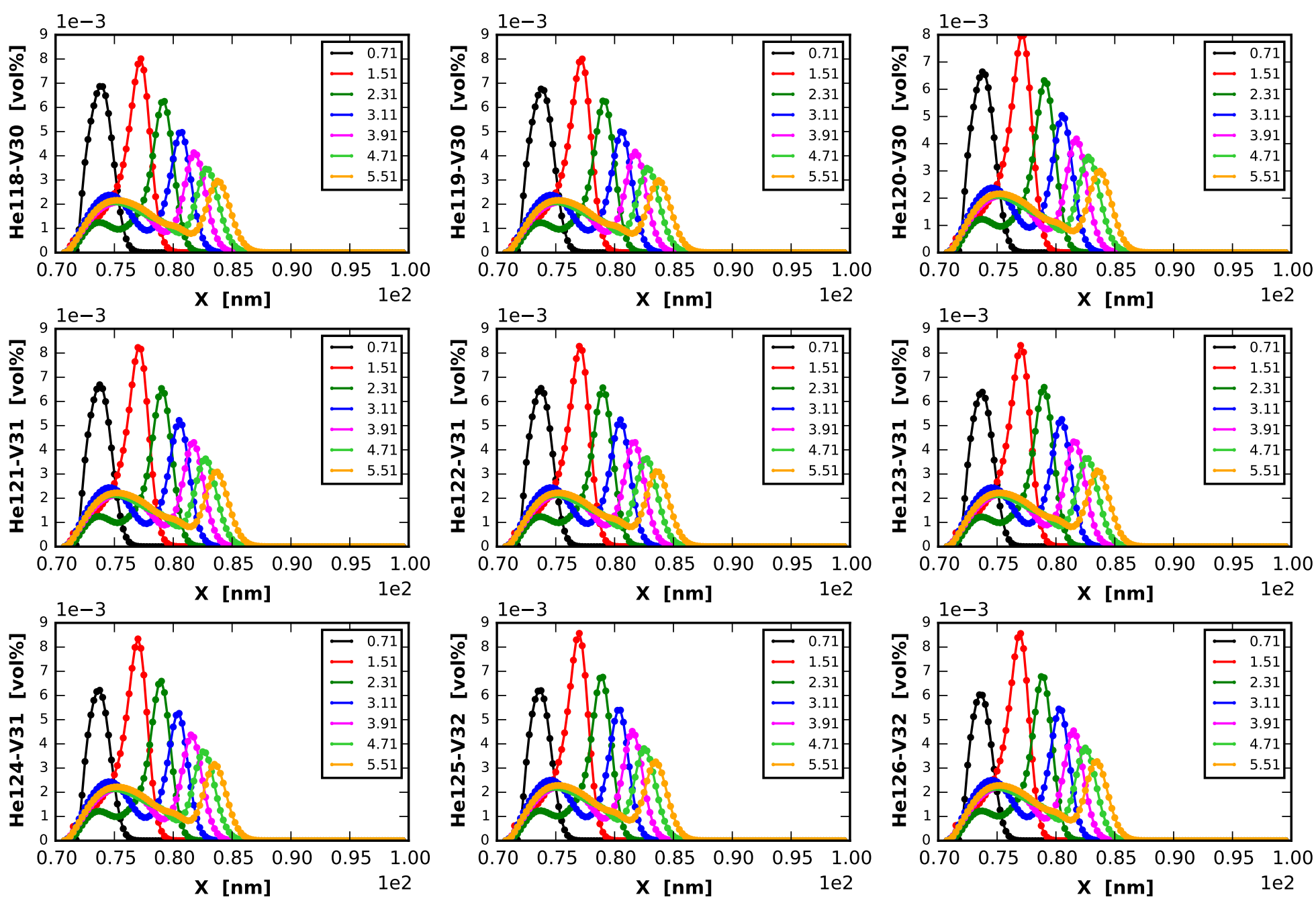
Transient: $[0.71,1.51,2.31,3.11,3.91,4.71,5.51]$ [us]
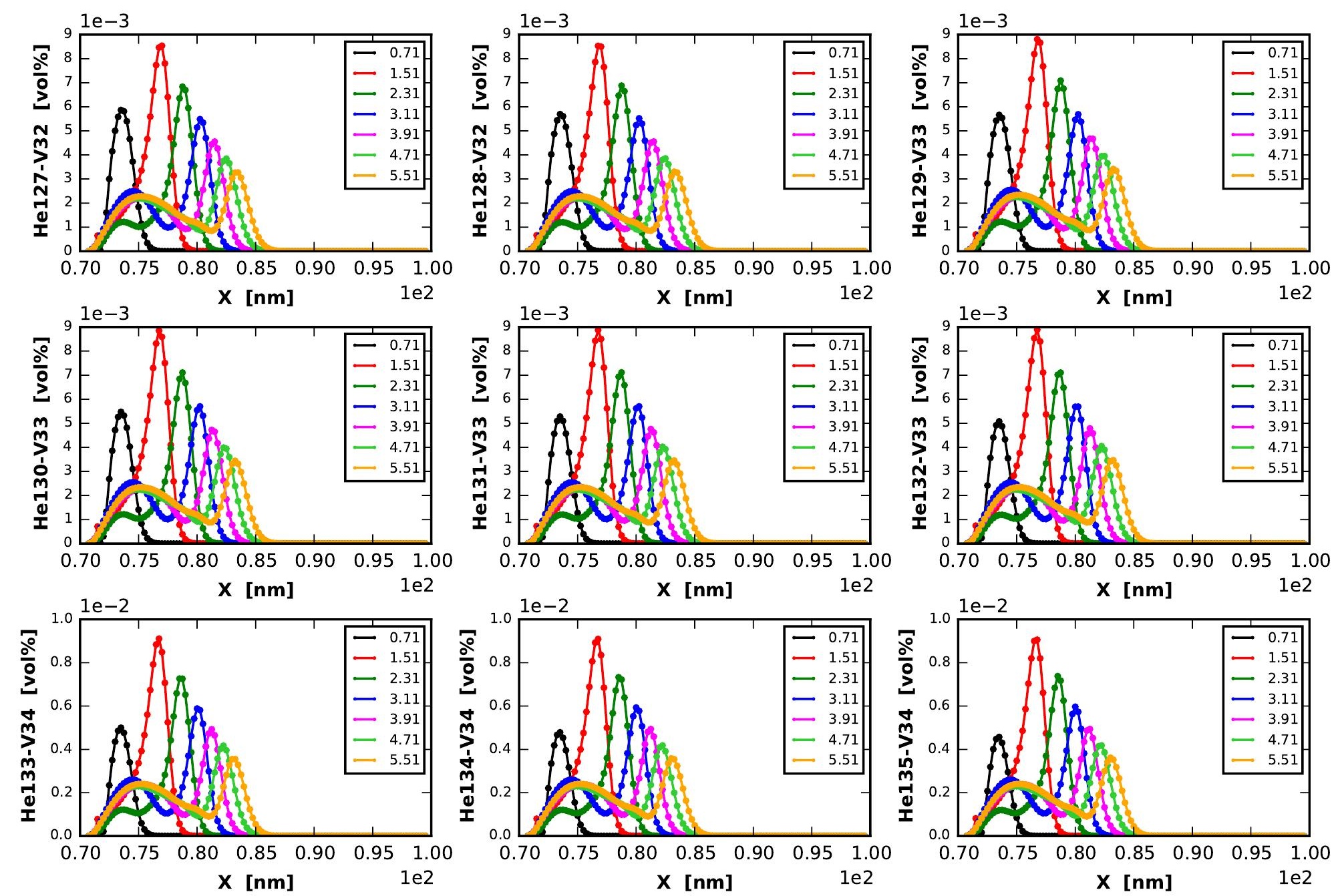


\section{Transient: [0.71, 1.51, 2.31, 3.11, 3.91, 4.71, 5.51] [us]}
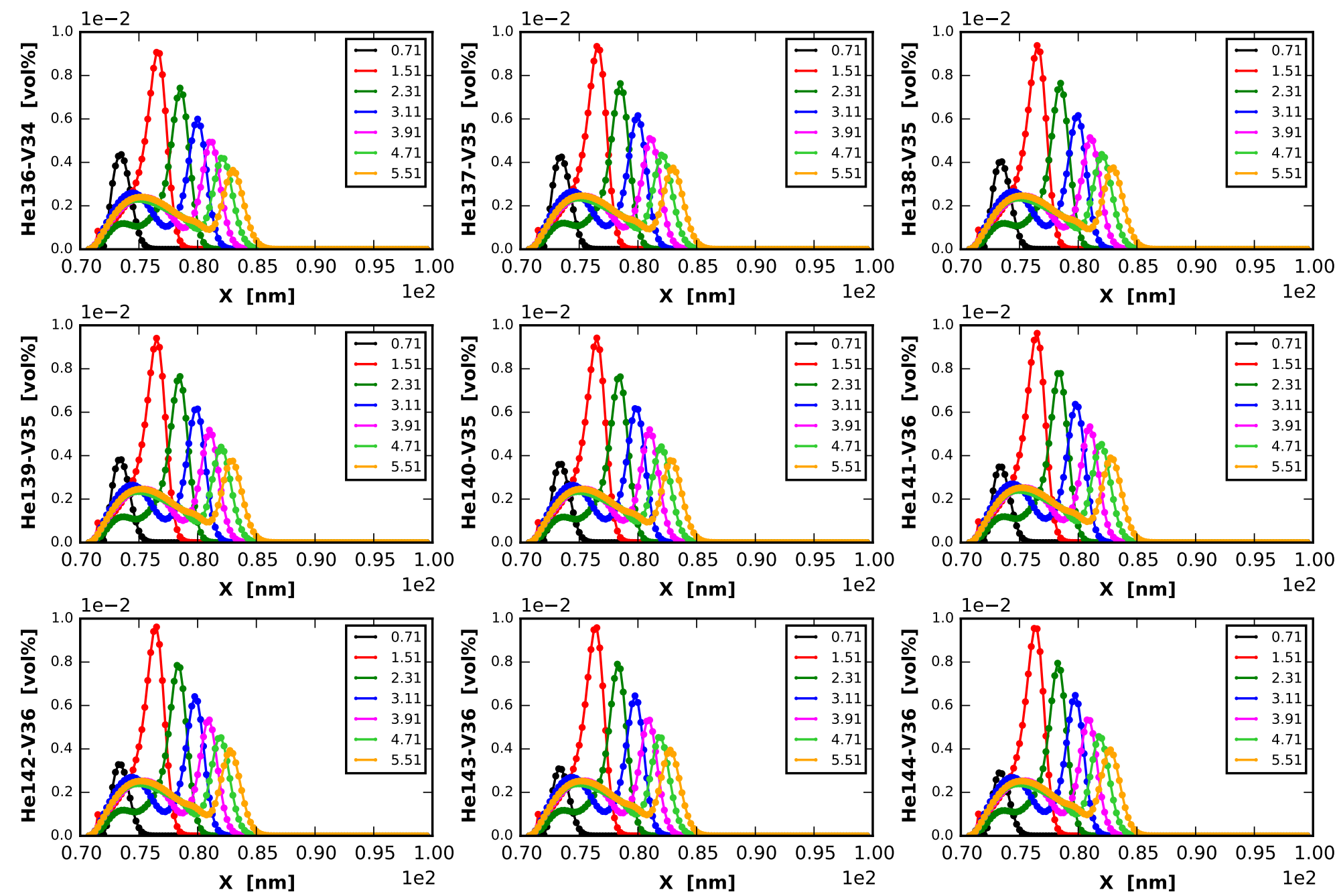
Transient: $[0.71,1.51,2.31,3.11,3.91,4.71,5.51]$ [us]
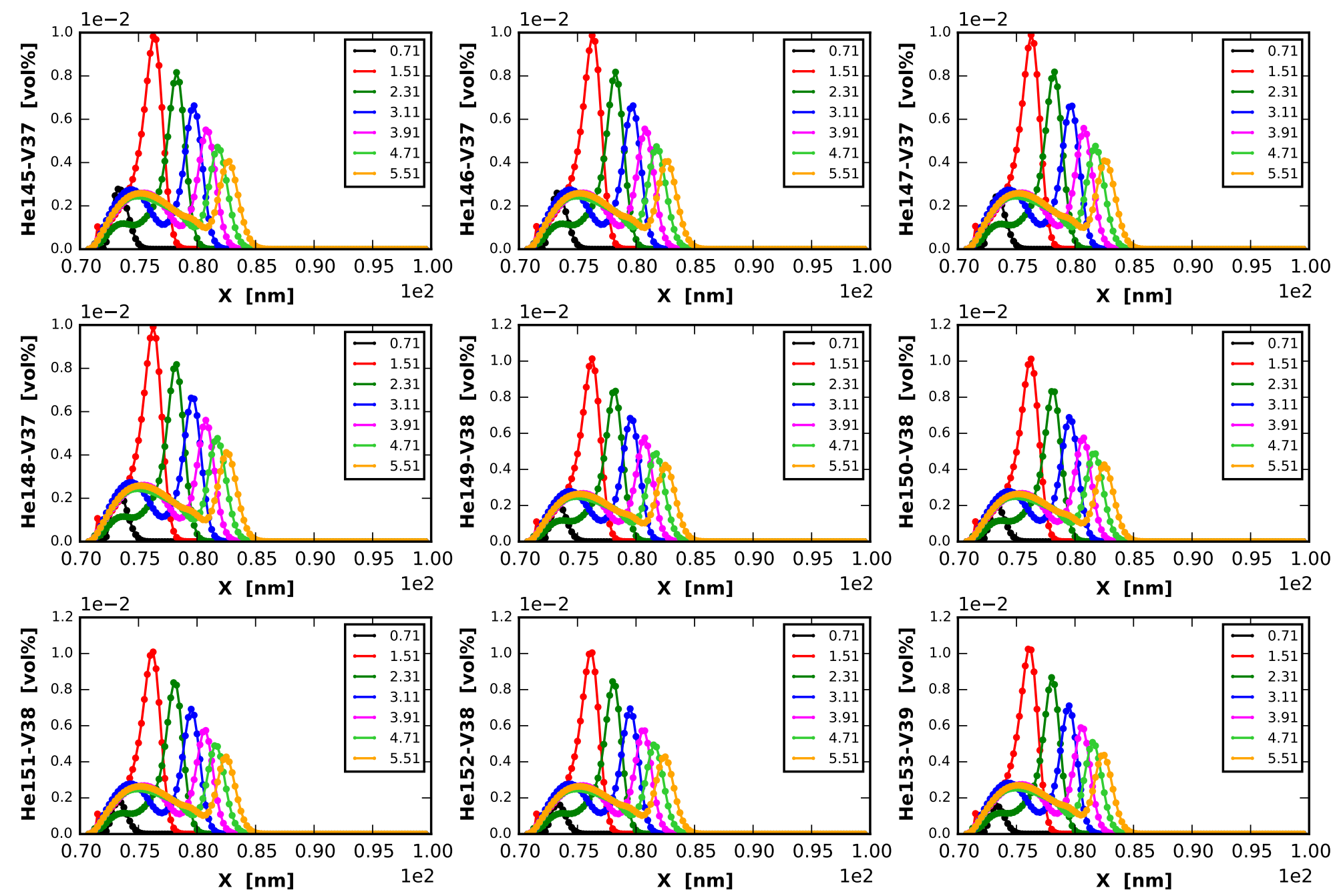
Transient: $[0.71,1.51,2.31,3.11,3.91,4.71,5.51]$ [us]
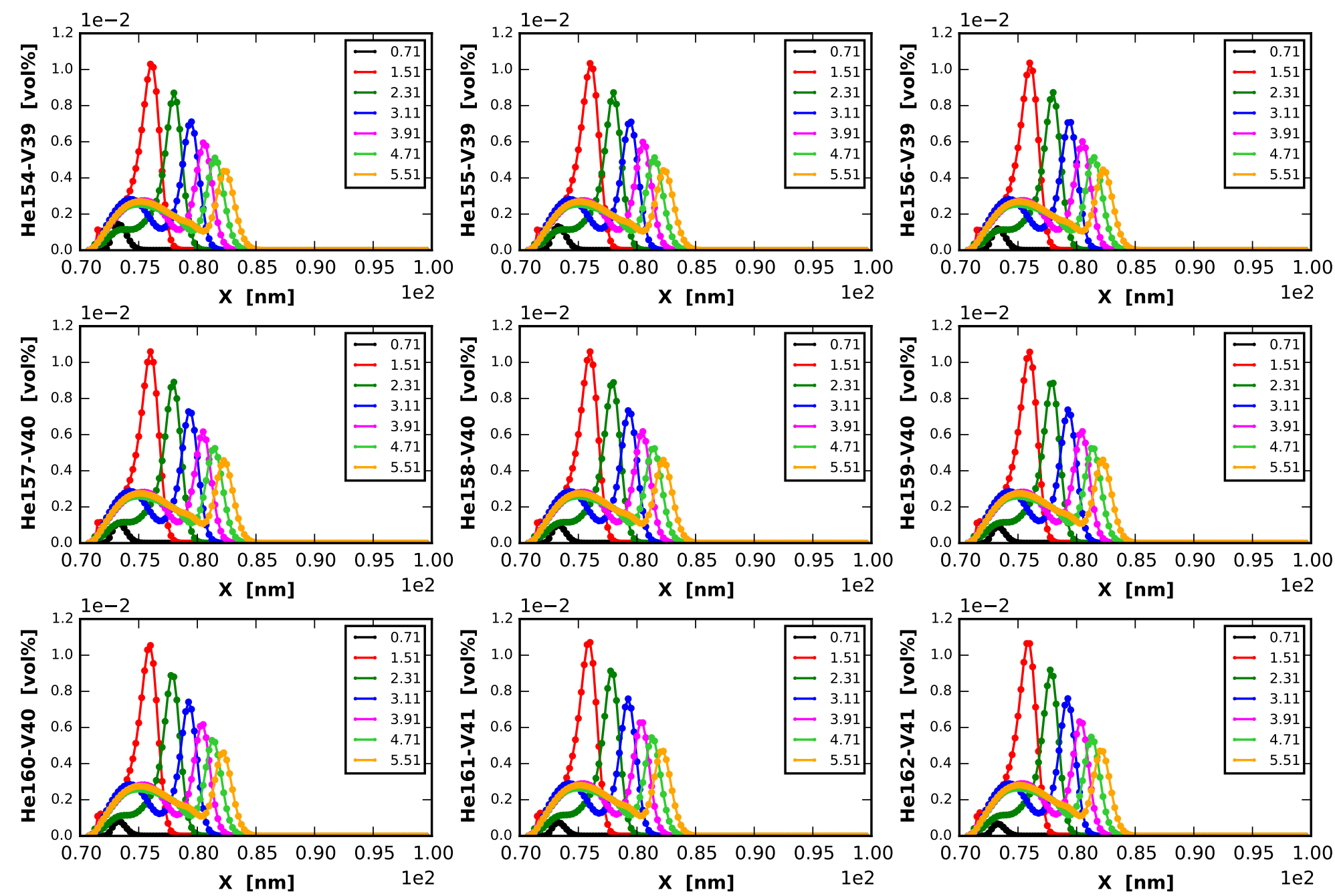
Transient: [0.71, 1.51, 2.31, 3.11, 3.91, 4.71, 5.51] [us]
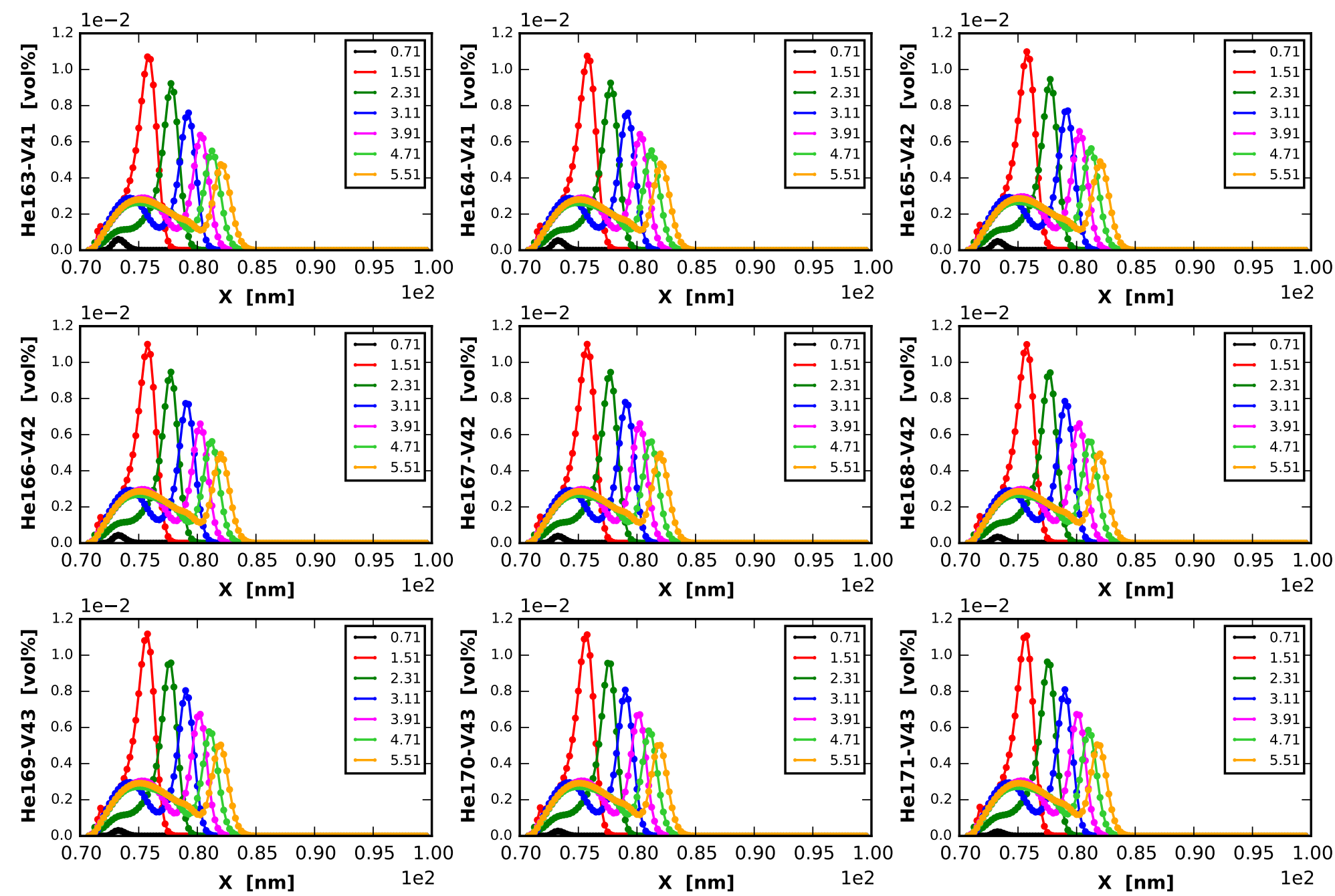
Transient: [0.71, 1.51, 2.31, 3.11, 3.91, 4.71, 5.51] [us]
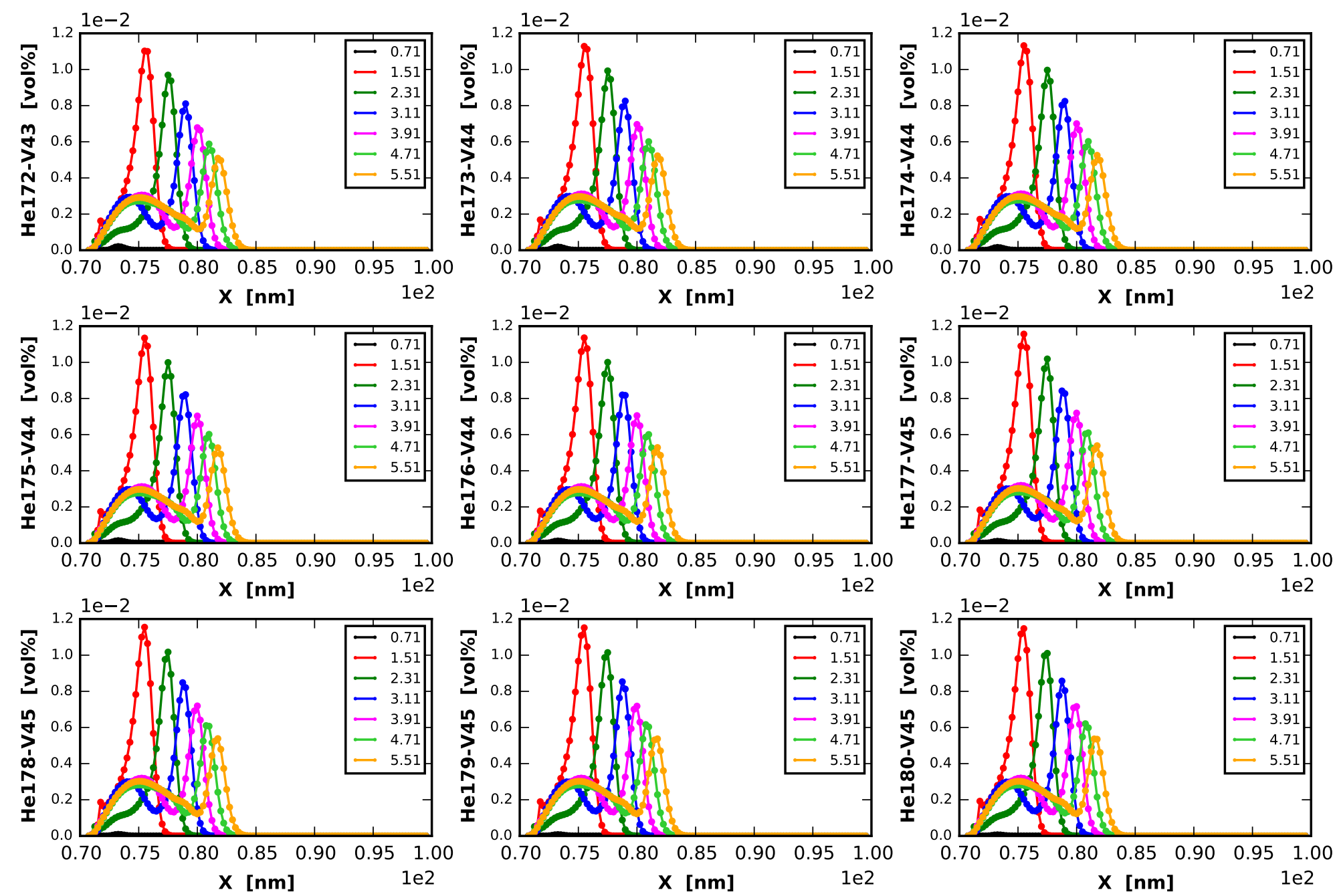
Transient: [0.71, 1.51, 2.31, 3.11, 3.91, 4.71, 5.51] [us]
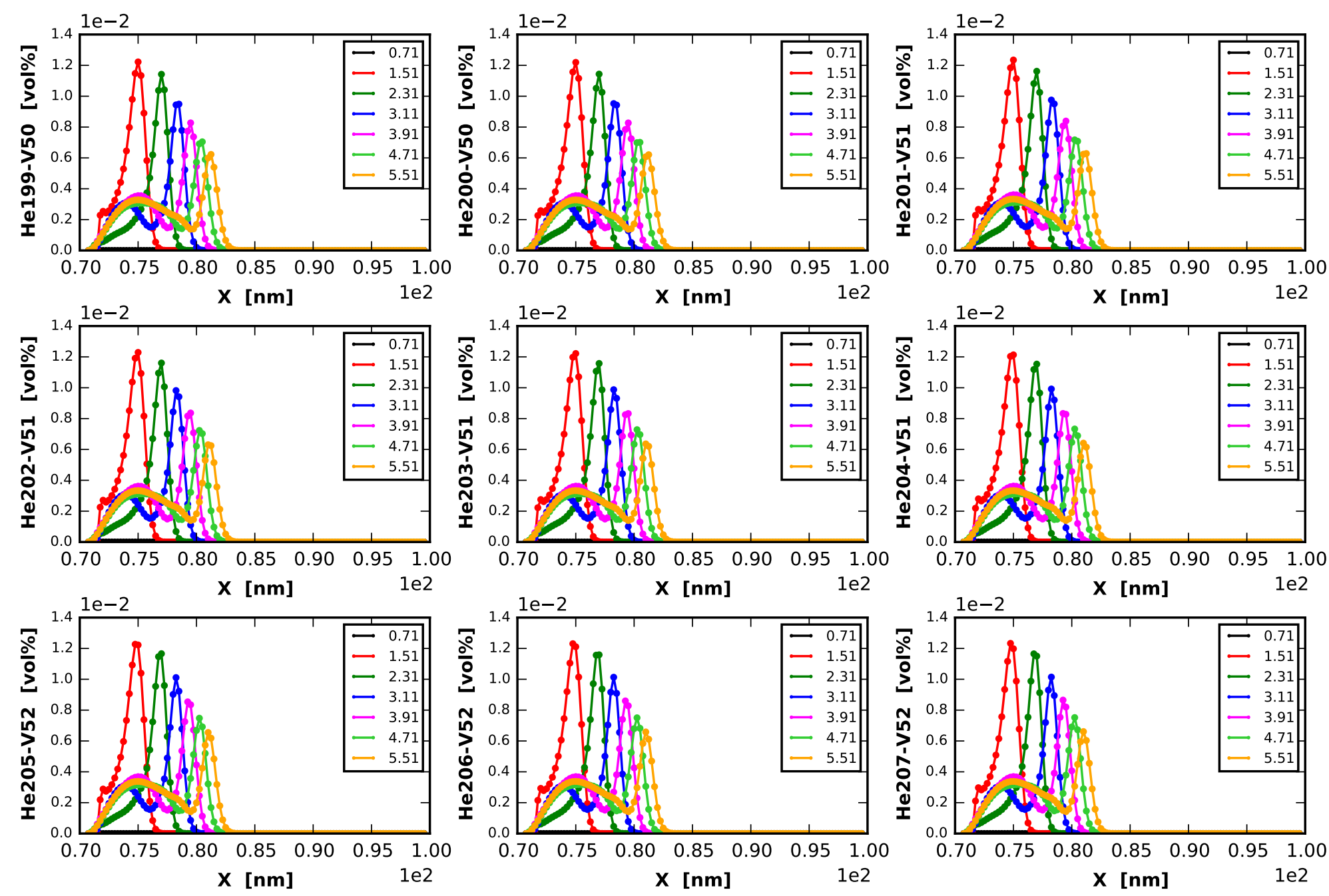
Transient: $[0.71,1.51,2.31,3.11,3.91,4.71,5.51]$ [us]
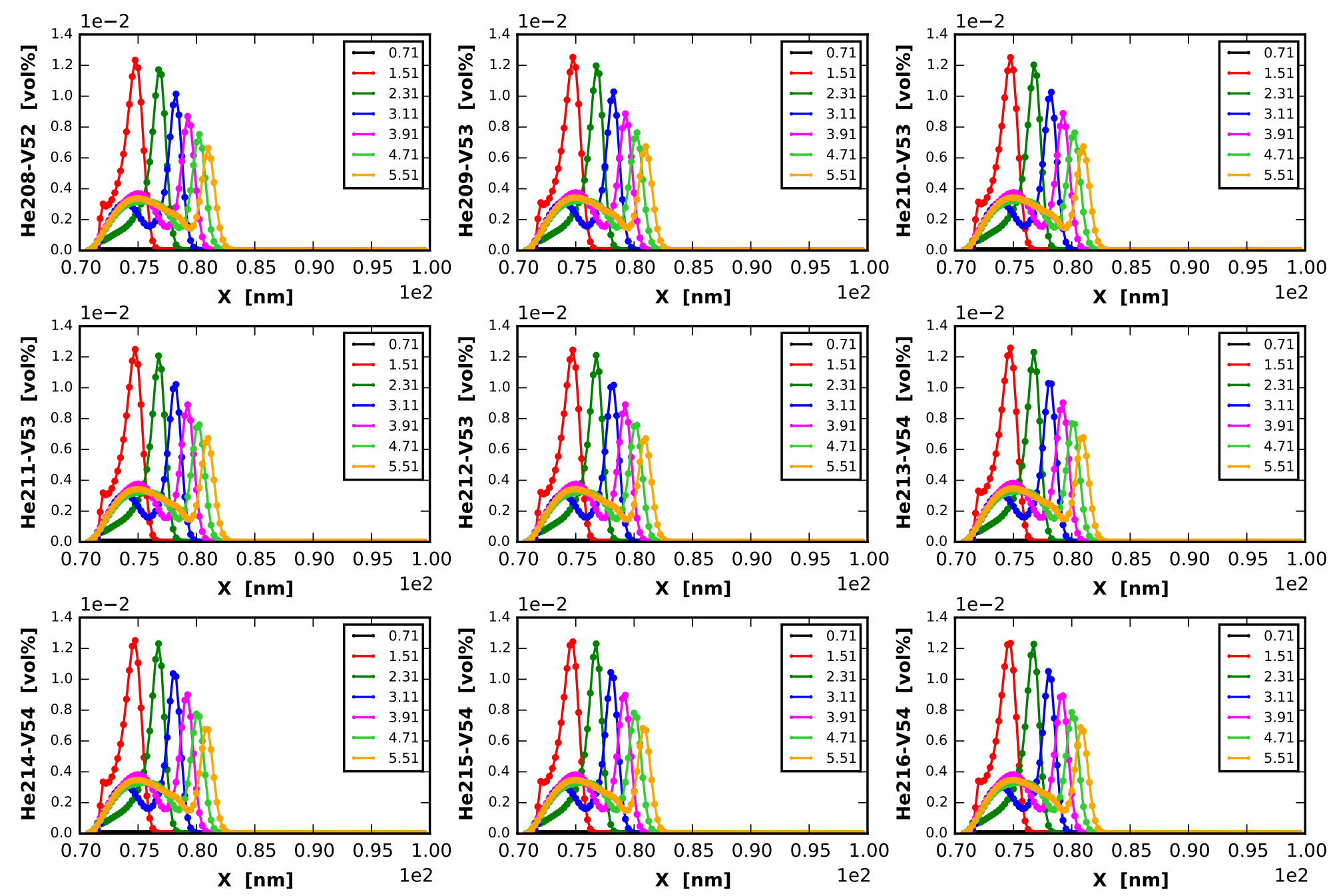
Transient: [0.71, 1.51, 2.31, 3.11, 3.91, 4.71, 5.51] [us]
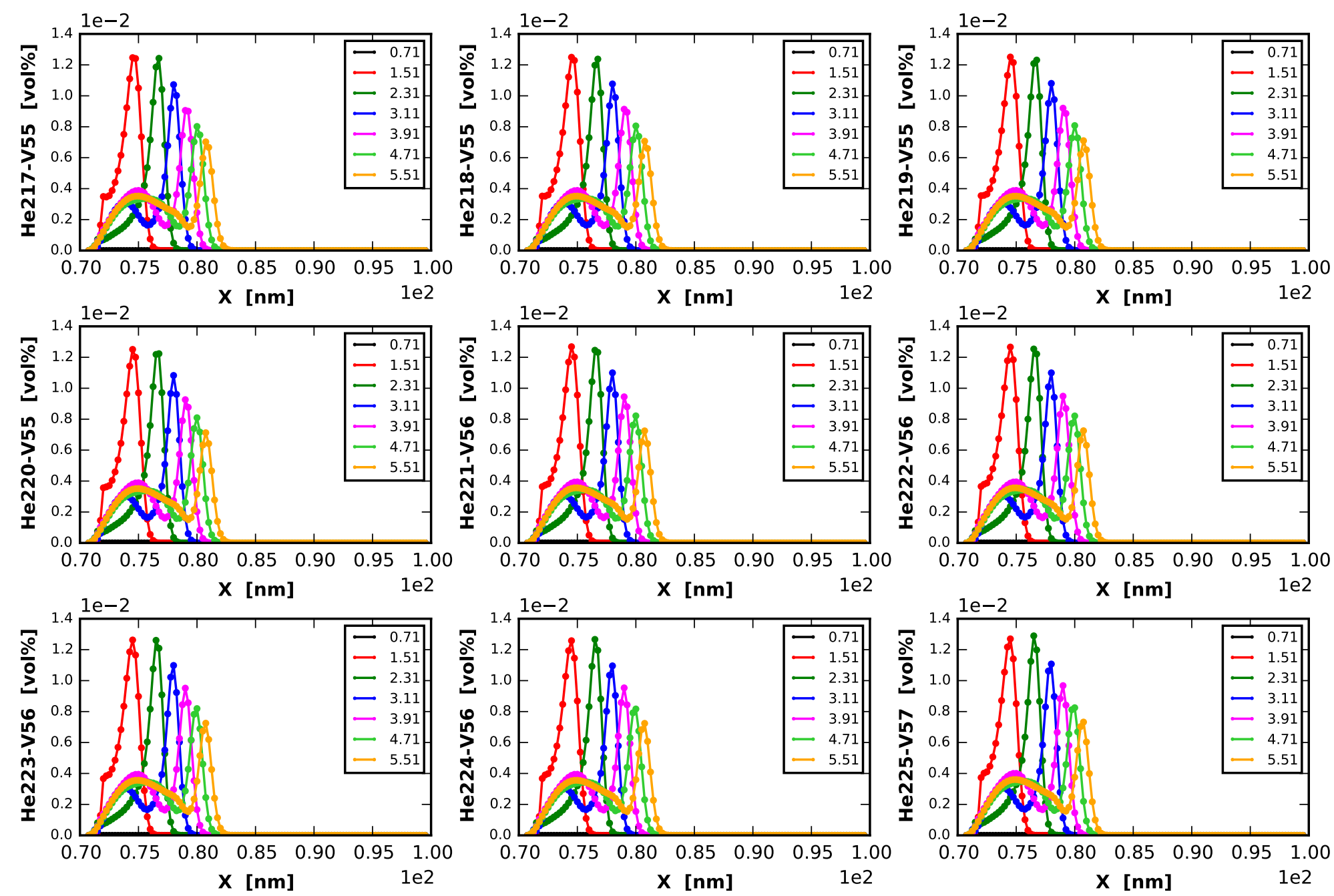
Transient: [0.71, 1.51, 2.31, 3.11, 3.91, 4.71, 5.51] [us]
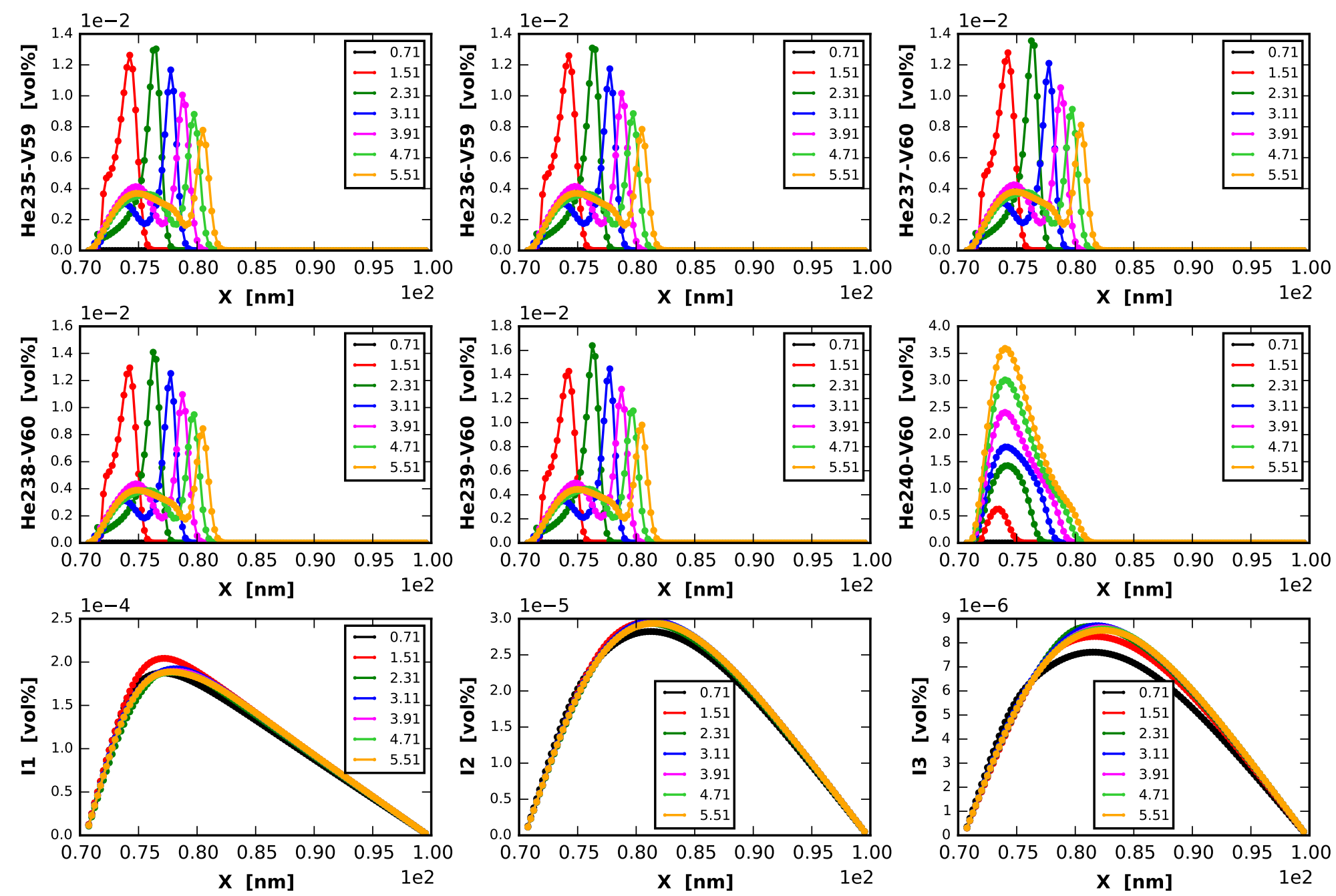
Transient: [0.71, 1.51, 2.31, 3.11, 3.91, 4.71, 5.51] [us]
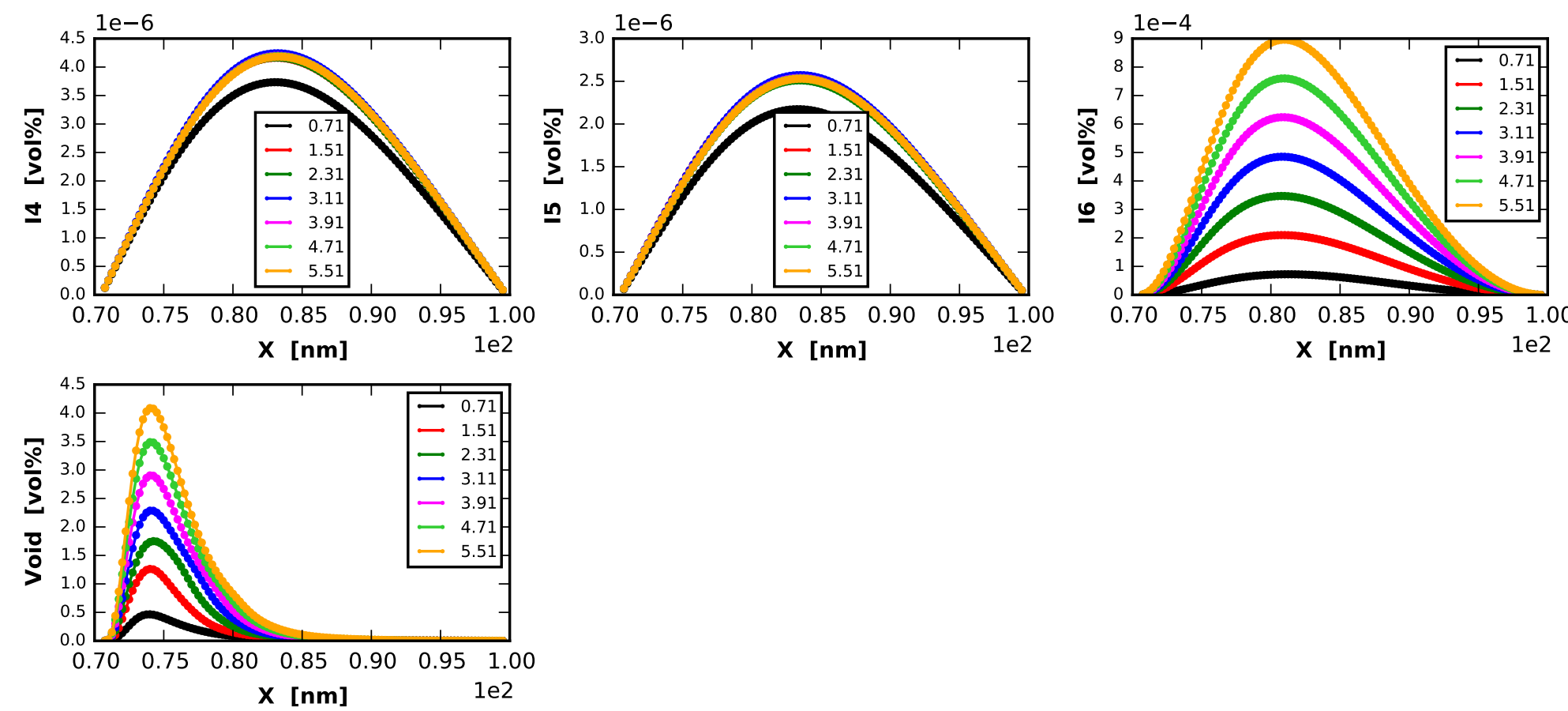
Bubble Nucleation, Growth and Coalescence ...

\section{A.2 Volume fraction plots (linear-log)}


Transient: [0.71, 1.51, 2.31, 3.11, 3.91, 4.71, 5.51] [us]
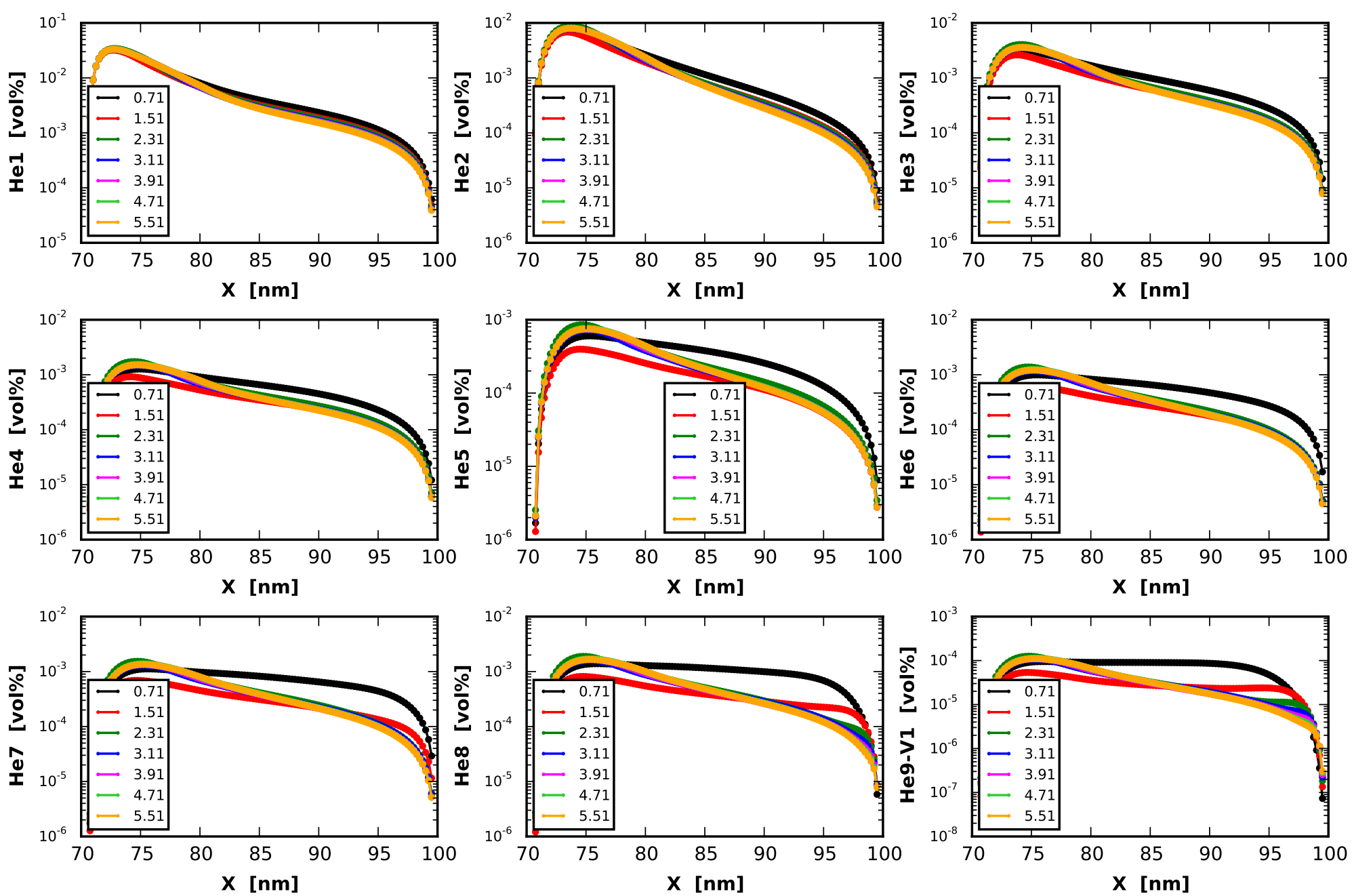
Transient: $[0.71,1.51,2.31,3.11,3.91,4.71,5.51]$ [us]
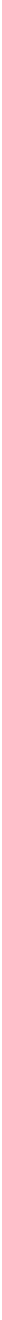
Transient: $[0.71,1.51,2.31,3.11,3.91,4.71,5.51]$ [us]
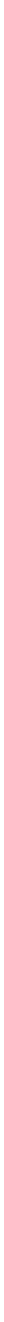
Transient: $[0.71,1.51,2.31,3.11,3.91,4.71,5.51]$ [us]
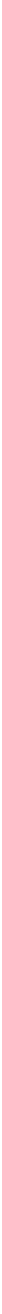
Transient: $[0.71,1.51,2.31,3.11,3.91,4.71,5.51]$ [us]
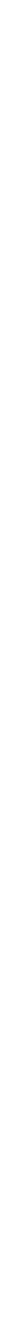


\section{A.3 Cumulative volume fraction plots}


Transient: $[0.71,1.51,2.31,3.11,3.91,4.71,5.51]$ [us]
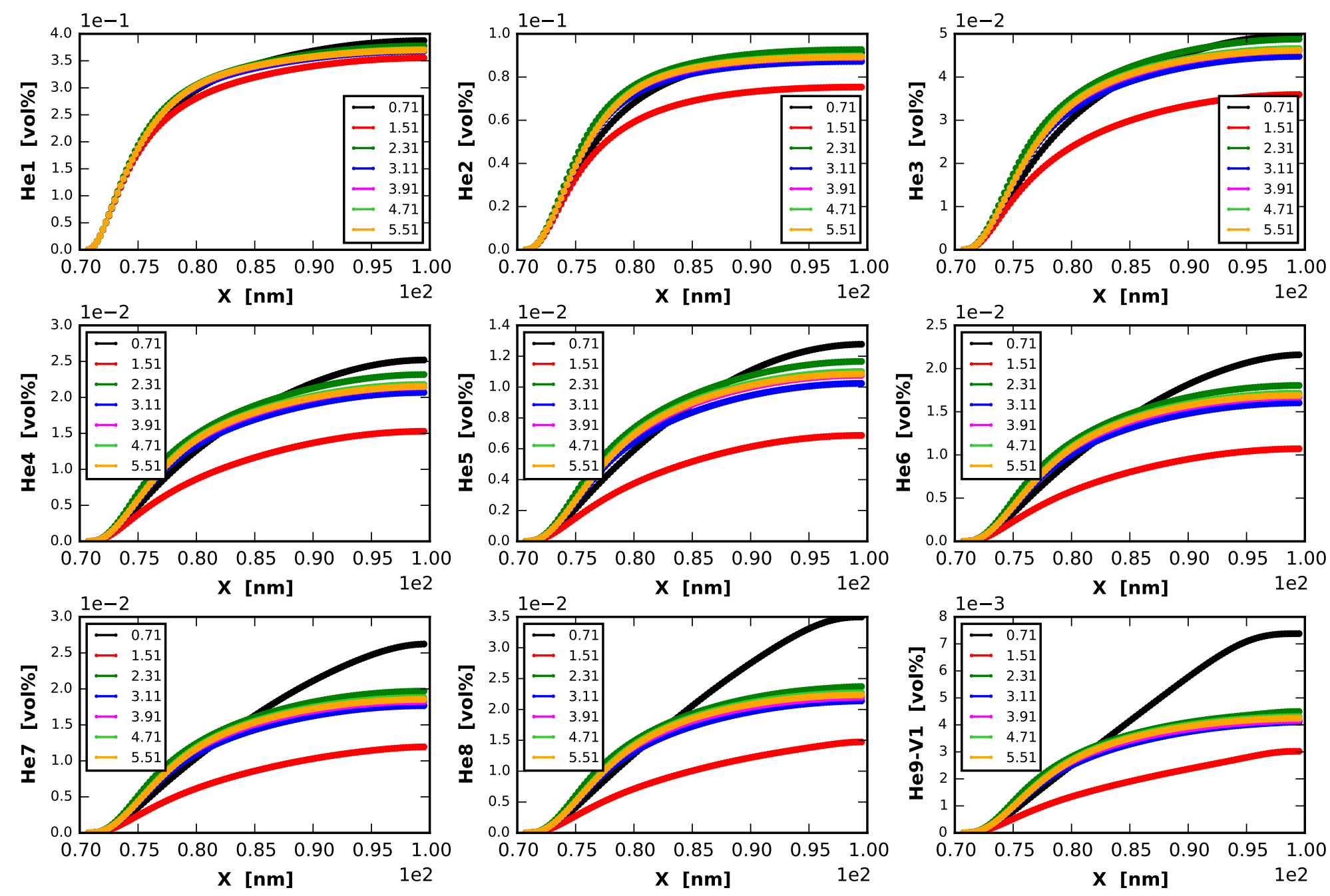
Transient: $[0.71,1.51,2.31,3.11,3.91,4.71,5.51]$ [us]
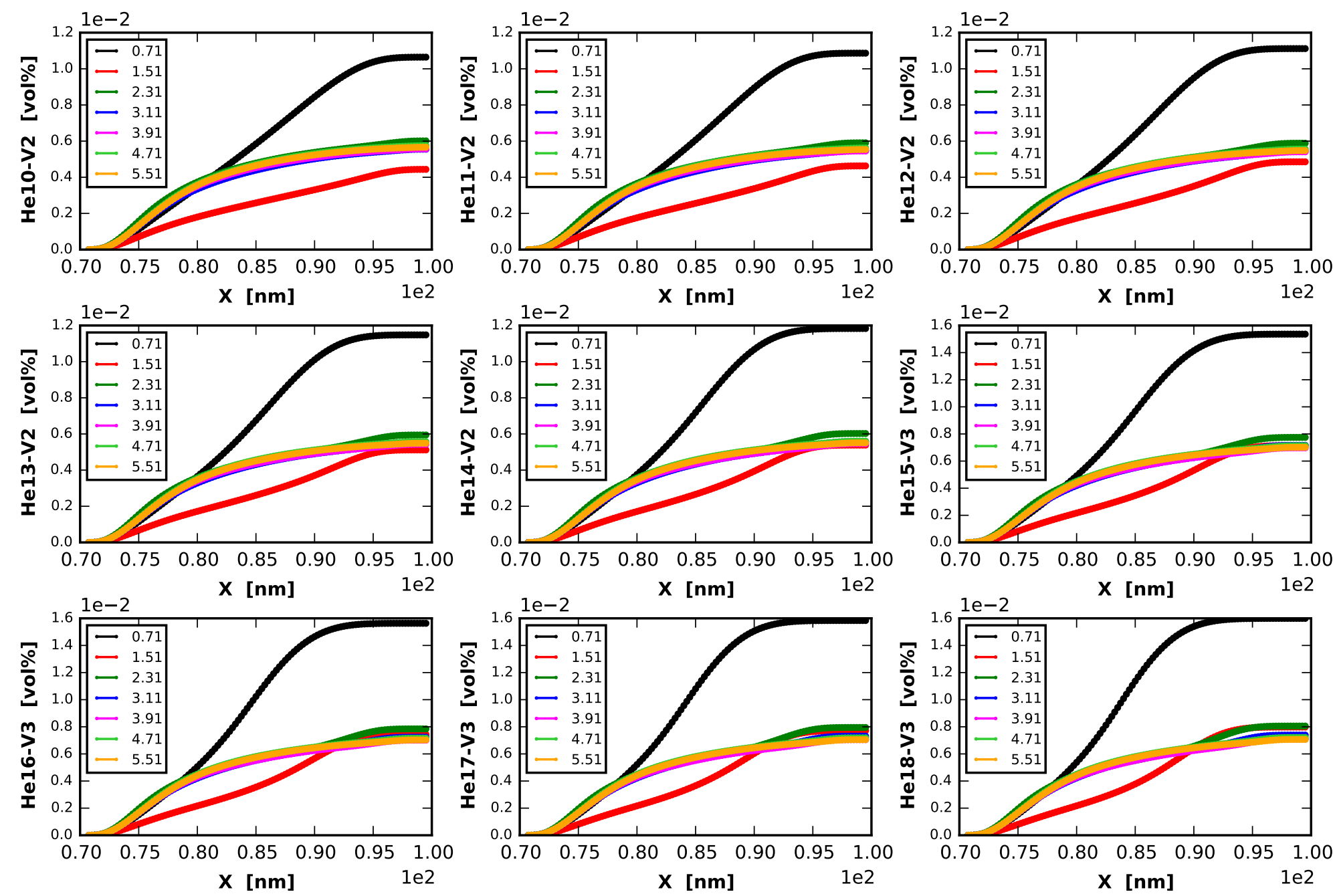
Transient: $[0.71,1.51,2.31,3.11,3.91,4.71,5.51]$ [us]
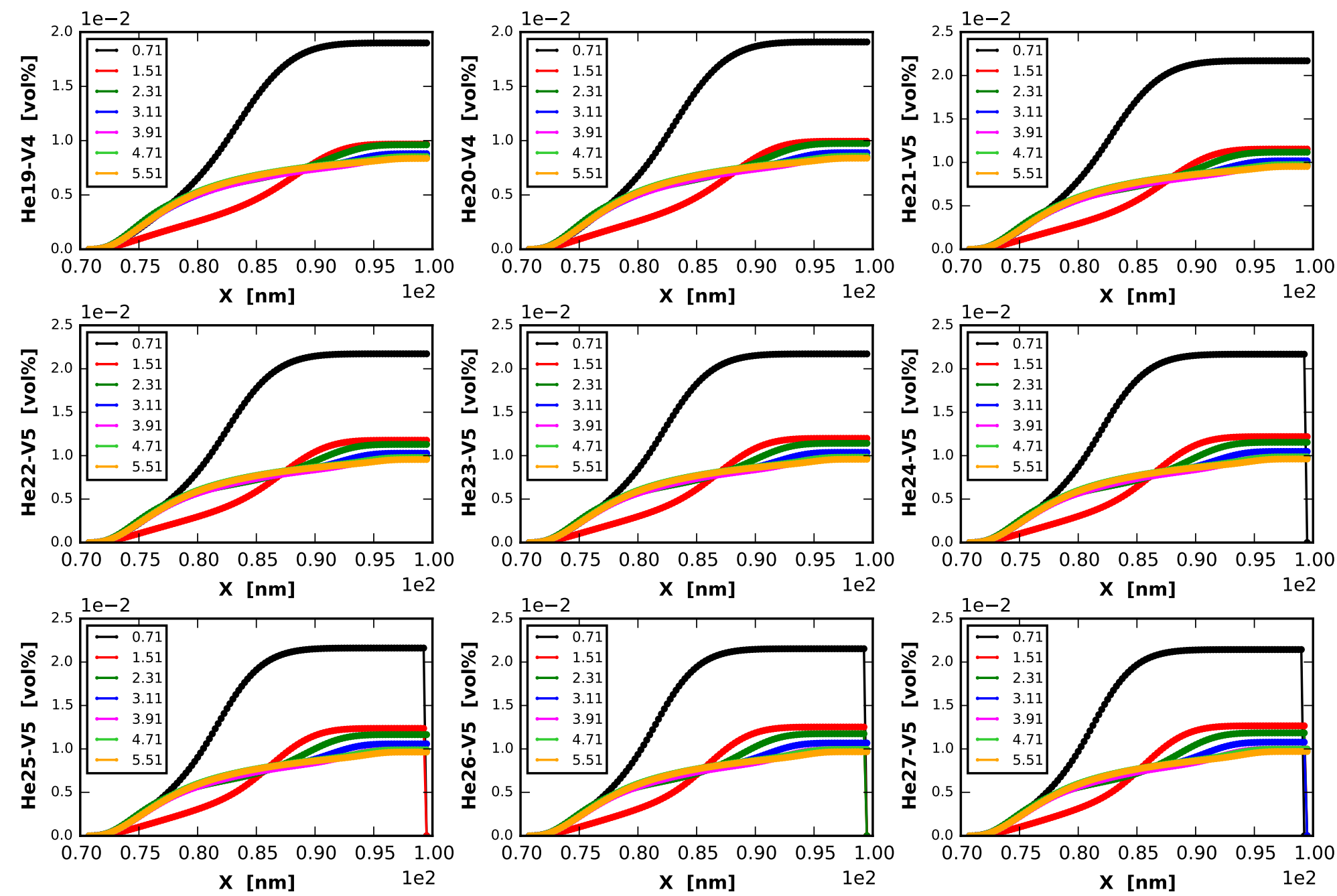
Transient: [0.71, 1.51, 2.31, 3.11, 3.91, 4.71, 5.51] [us]
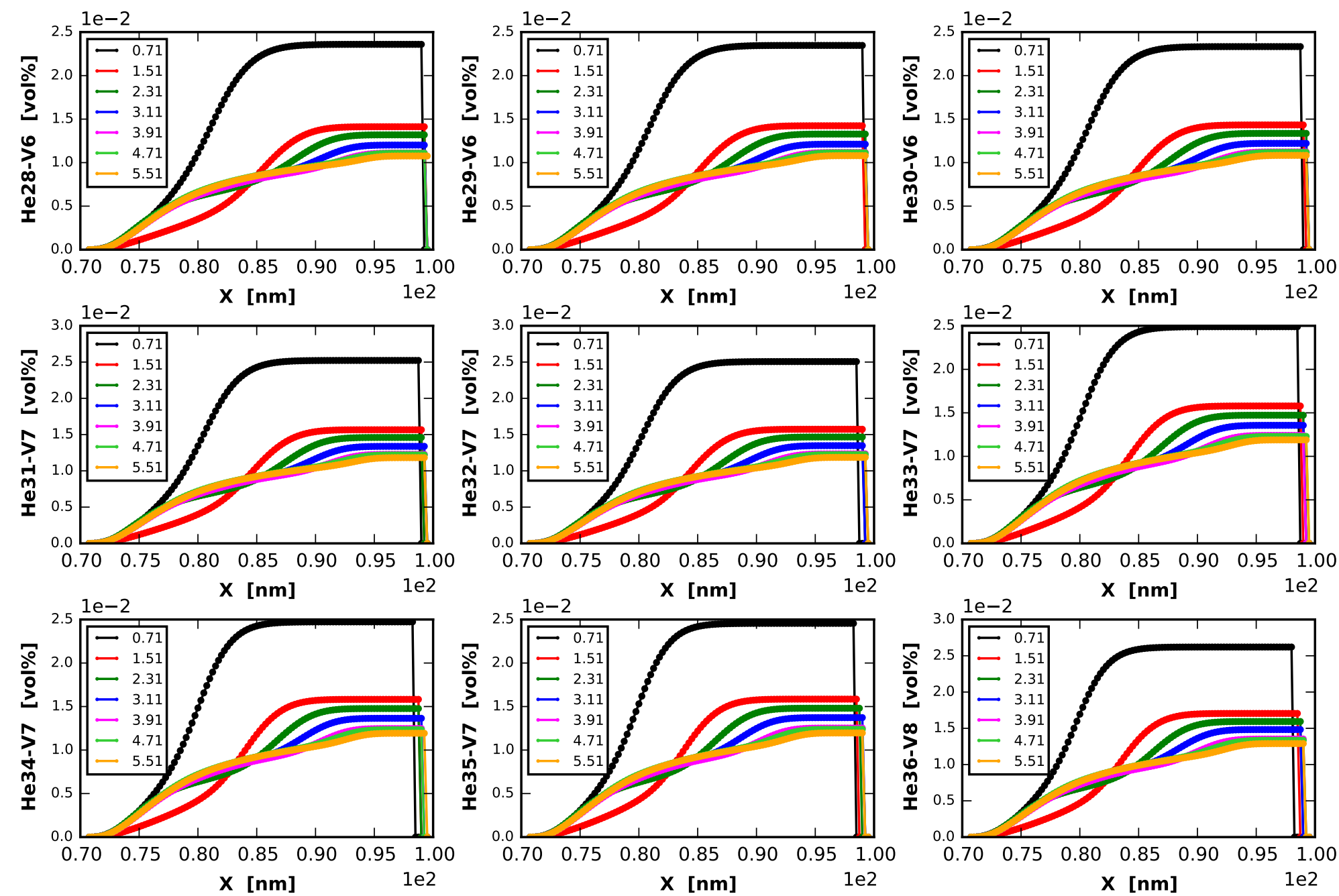
Transient: $[0.71,1.51,2.31,3.11,3.91,4.71,5.51]$ [us]
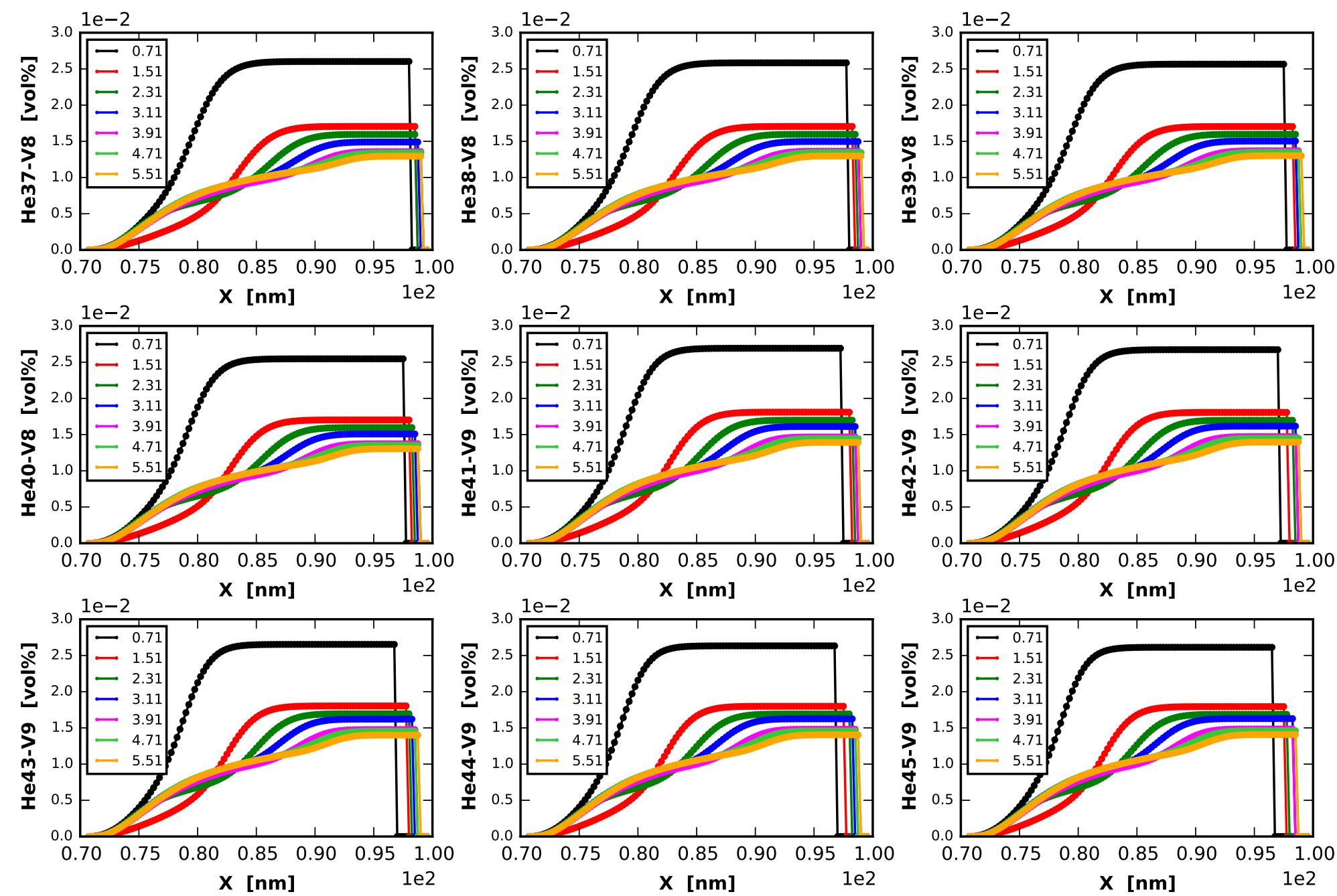
Transient: $[0.71,1.51,2.31,3.11,3.91,4.71,5.51]$ [us]
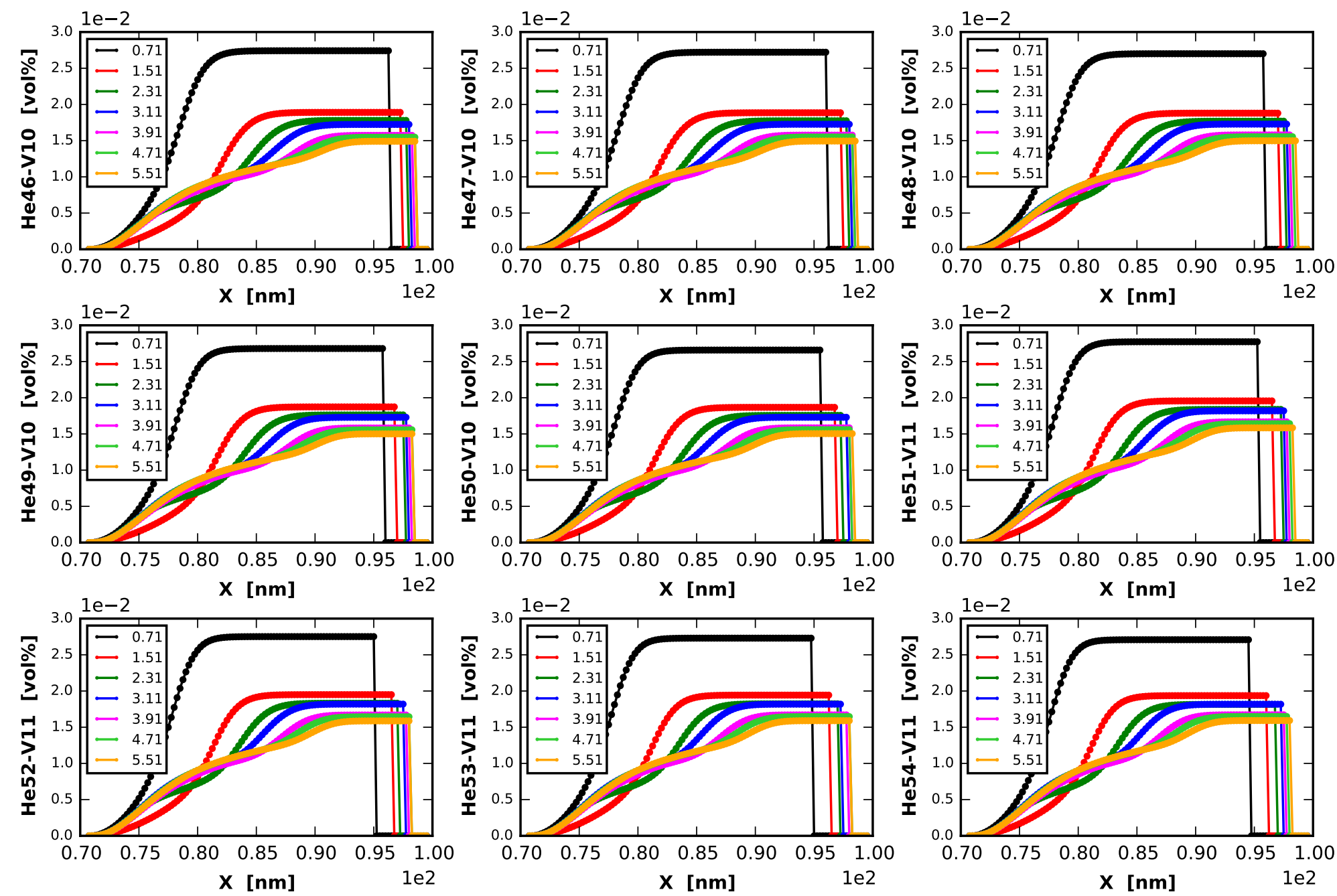
Transient: $[0.71,1.51,2.31,3.11,3.91,4.71,5.51]$ [us]
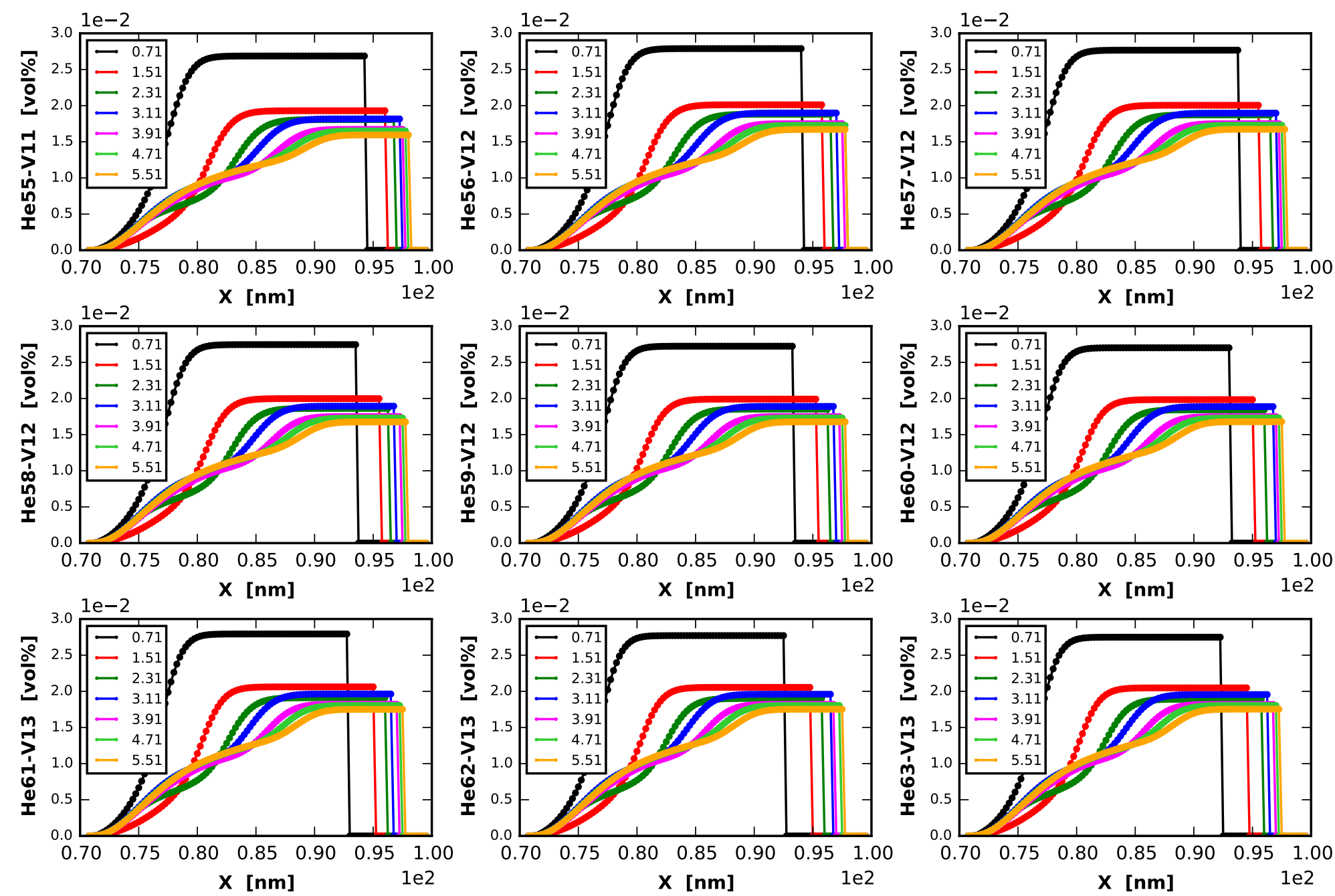
Transient: $[0.71,1.51,2.31,3.11,3.91,4.71,5.51]$ [us]
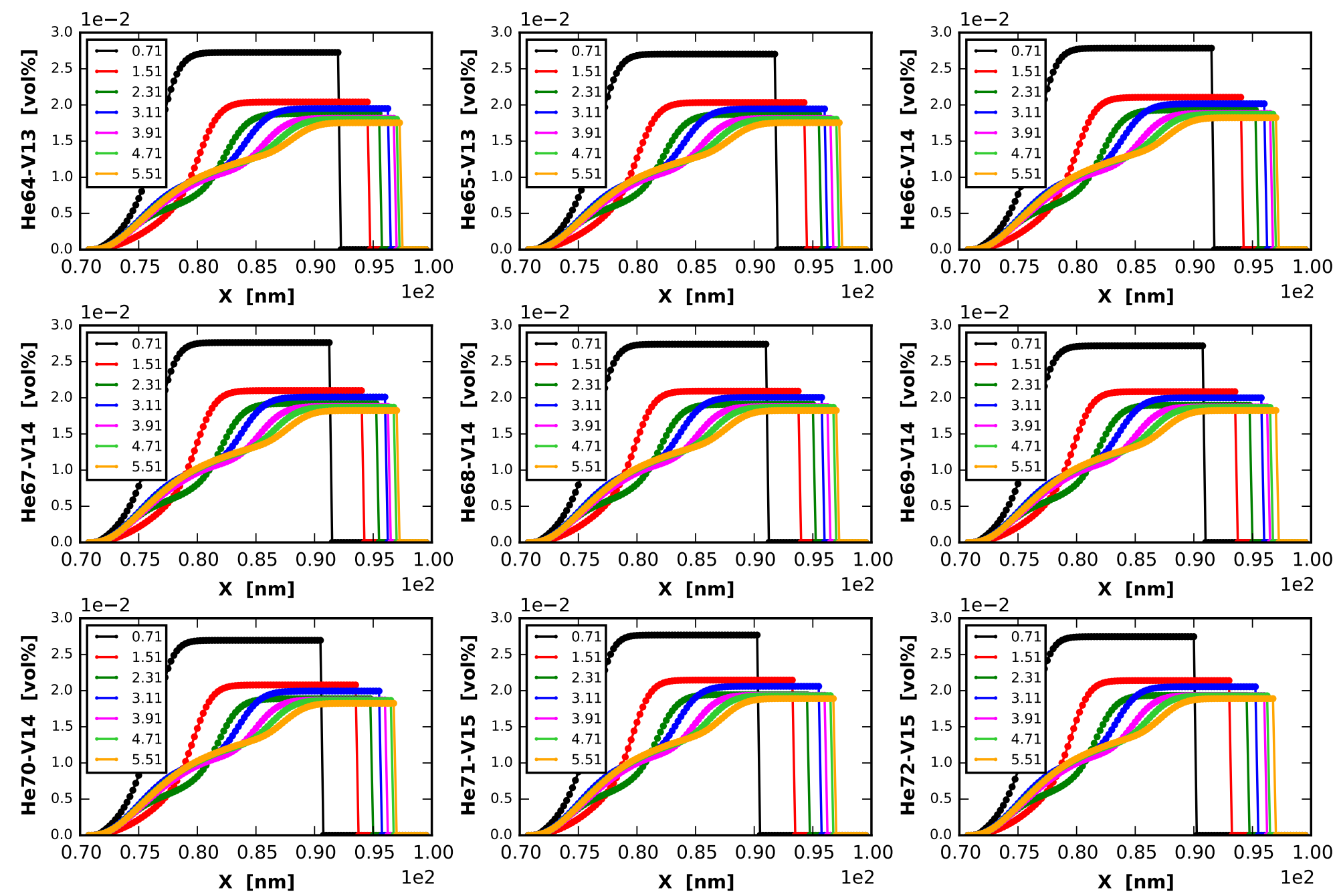
Transient: $[0.71,1.51,2.31,3.11,3.91,4.71,5.51]$ [us]
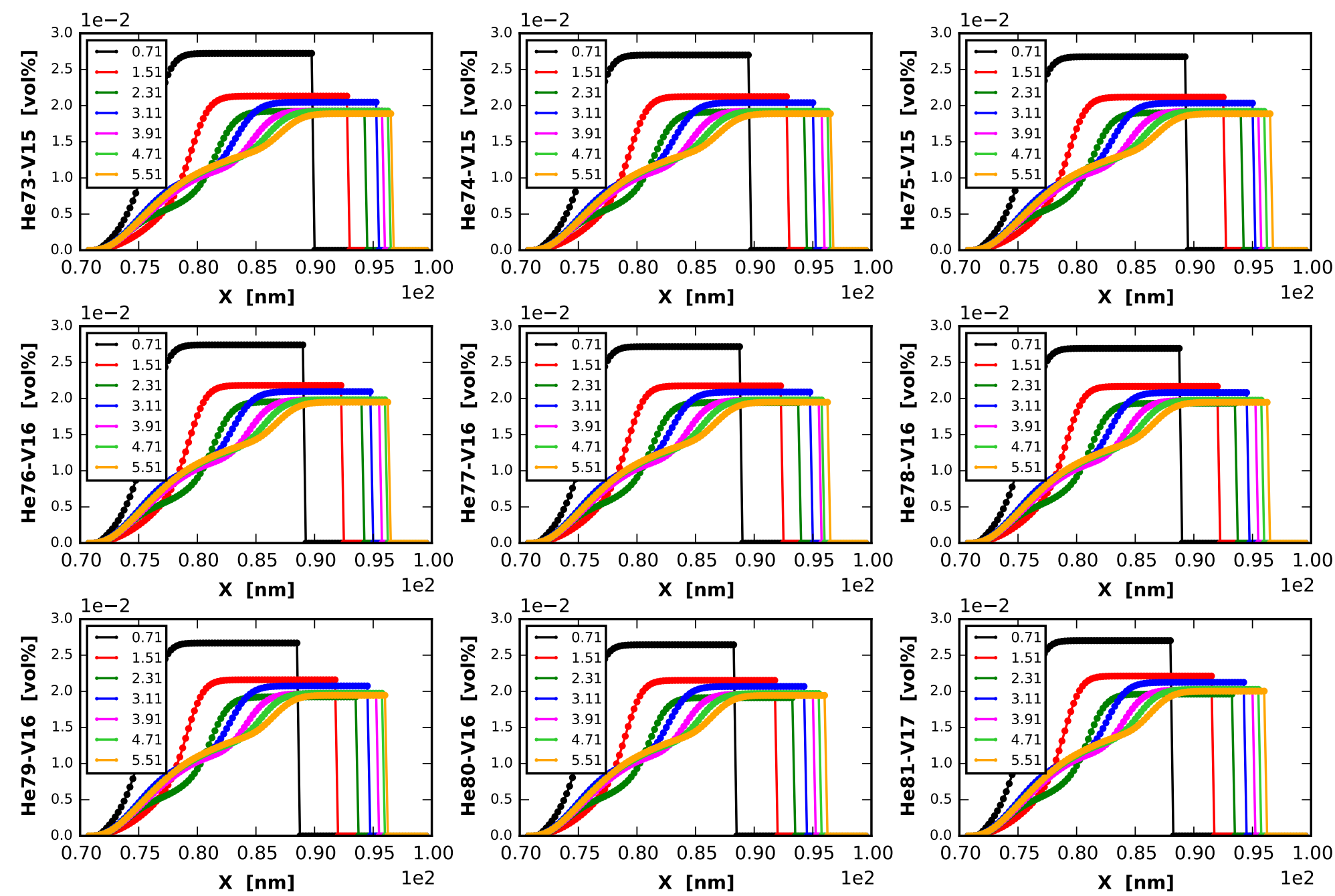


\section{Transient: [0.71, 1.51, 2.31, 3.11, 3.91, 4.71, 5.51] [us]}
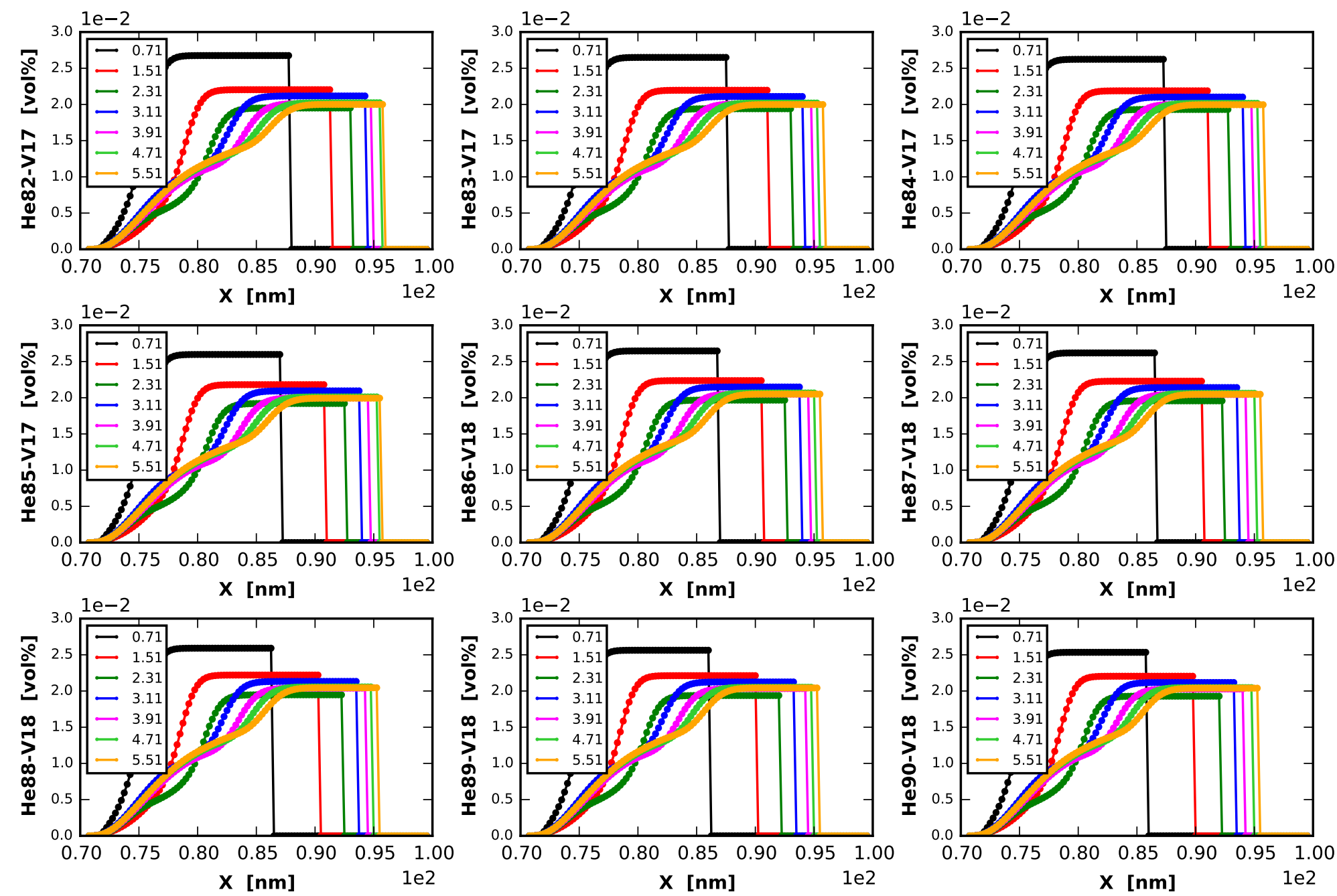
Transient: $[0.71,1.51,2.31,3.11,3.91,4.71,5.51]$ [us]
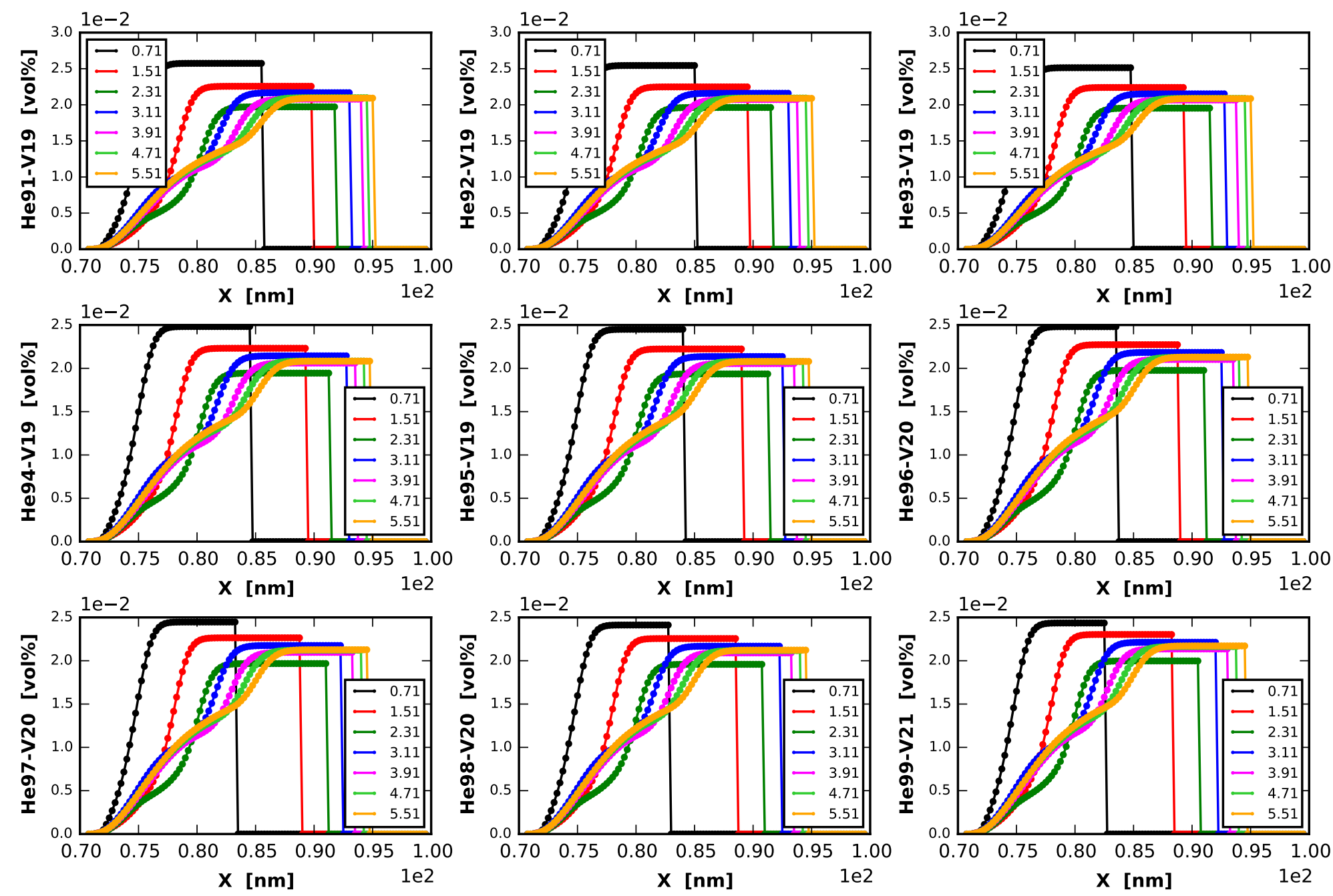
Transient: $[0.71,1.51,2.31,3.11,3.91,4.71,5.51]$ [us]
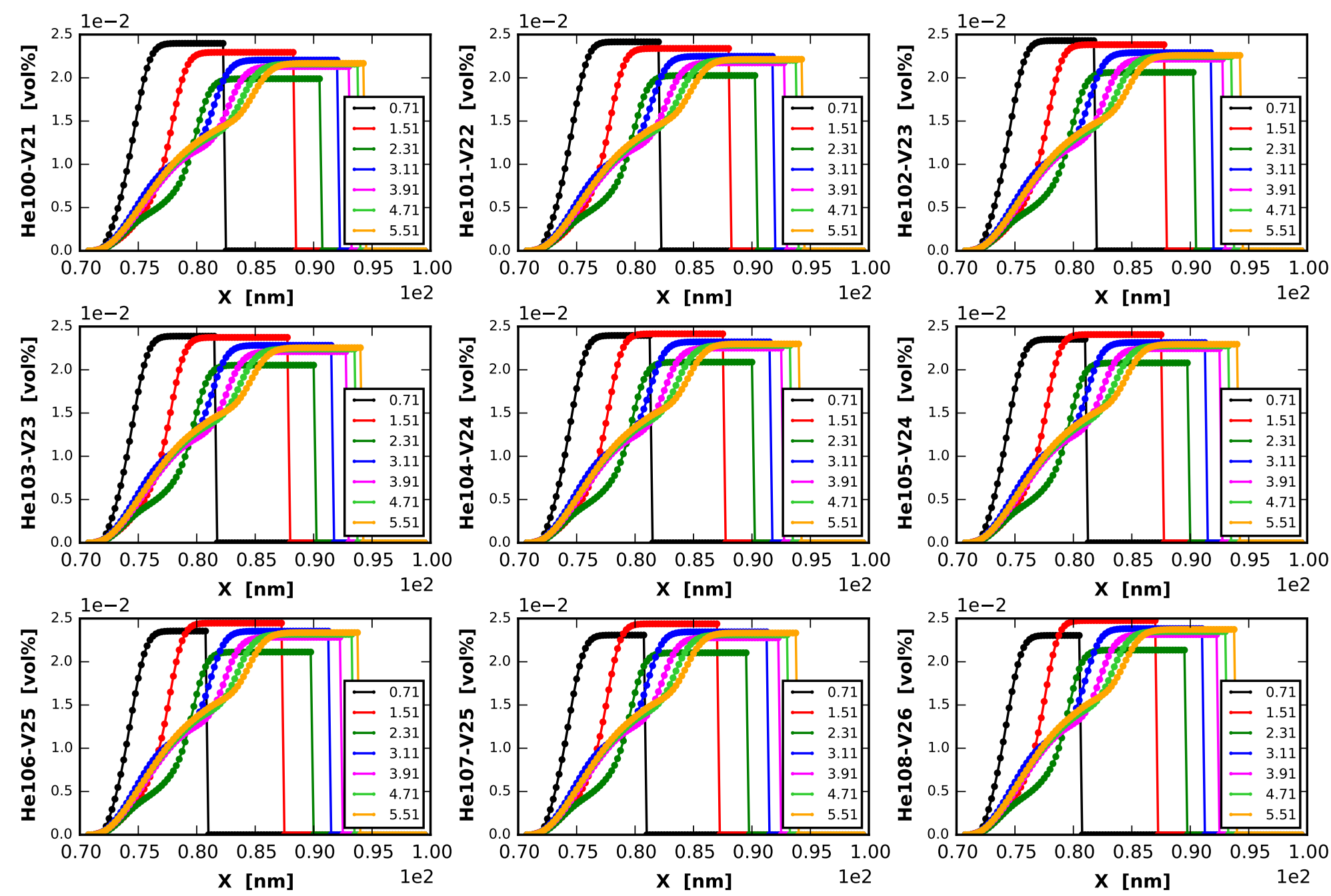
Transient: $[0.71,1.51,2.31,3.11,3.91,4.71,5.51]$ [us]
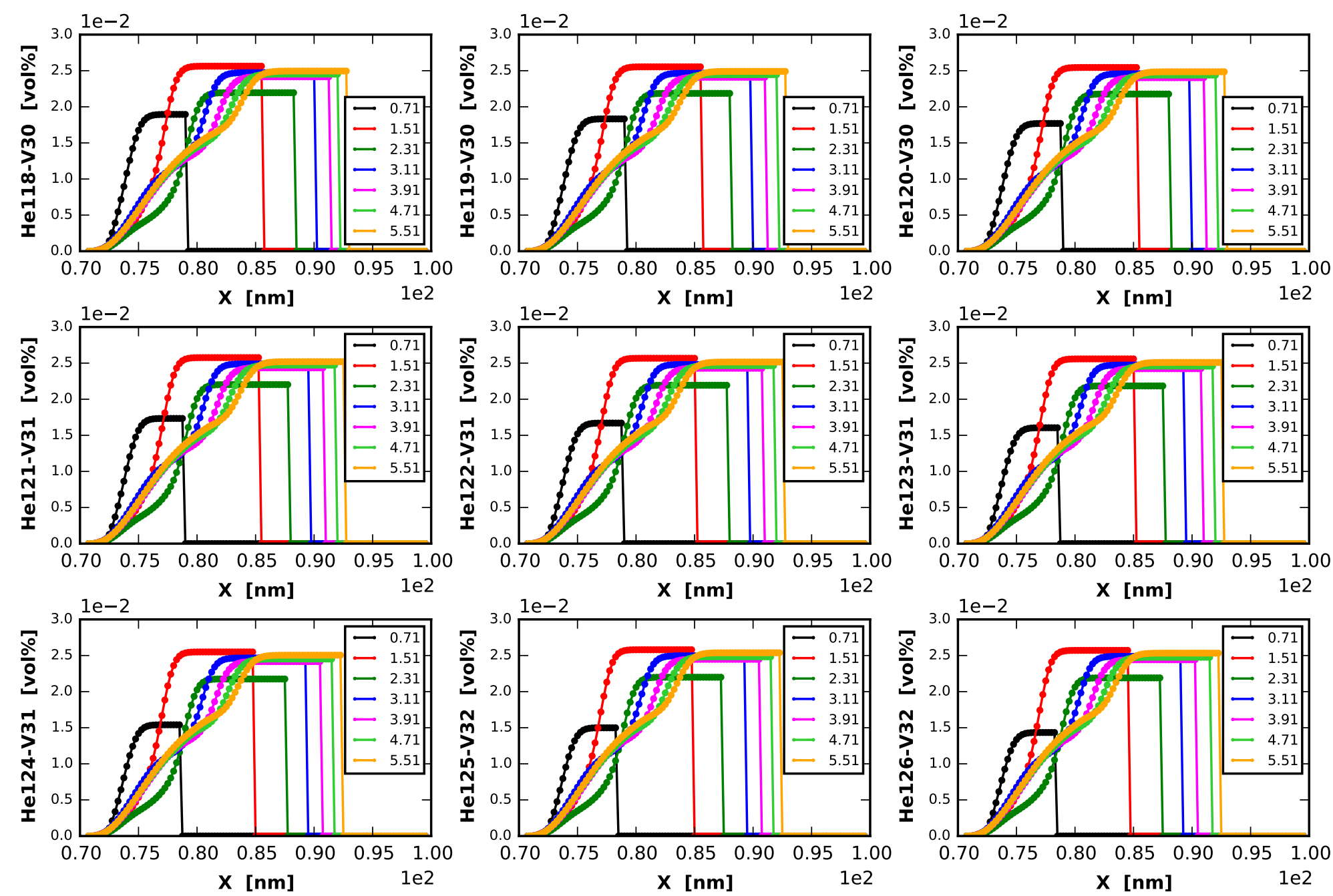
Transient: $[0.71,1.51,2.31,3.11,3.91,4.71,5.51]$ [us]
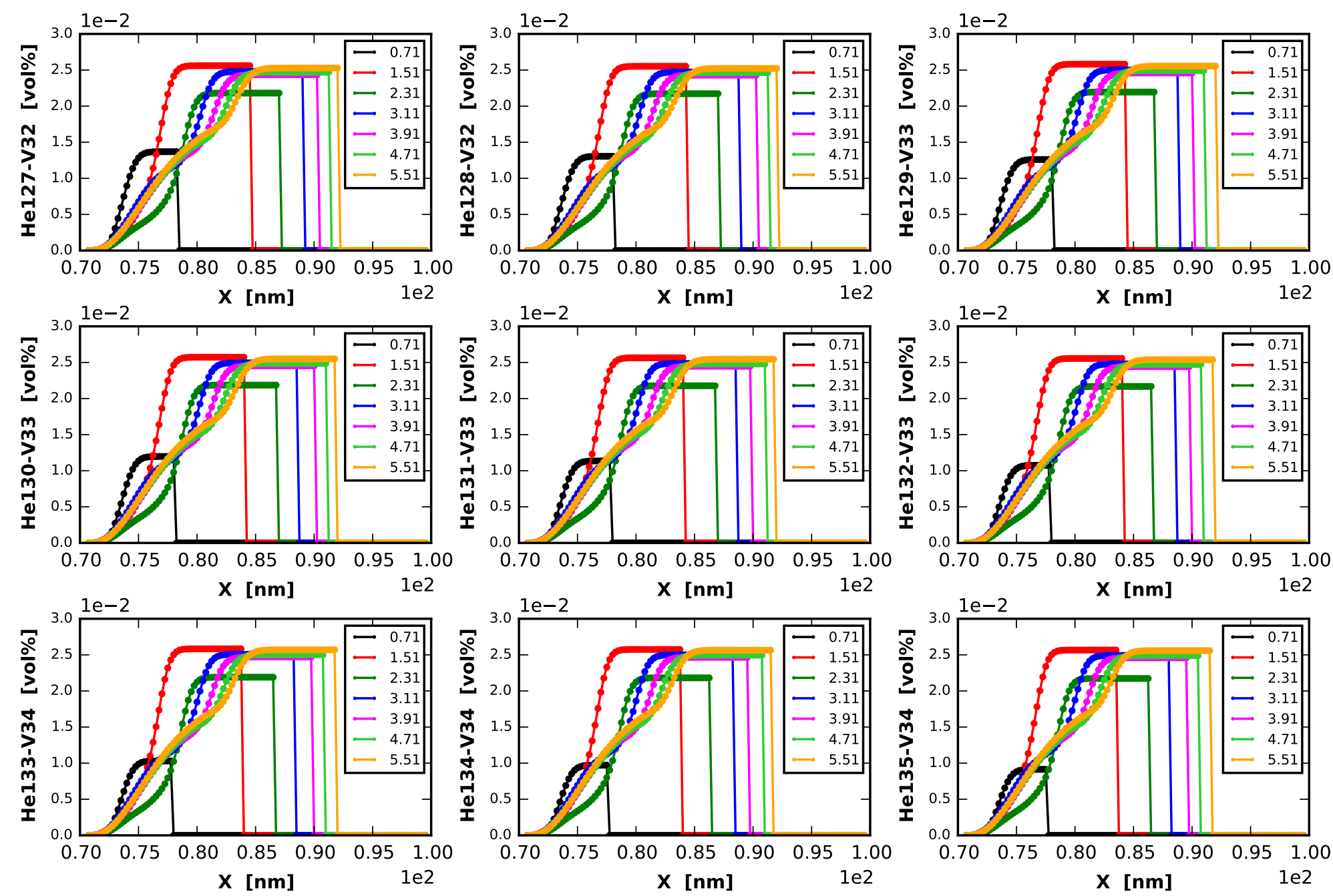


\section{Transient: $[0.71,1.51,2.31,3.11,3.91,4.71,5.51]$ [us]}
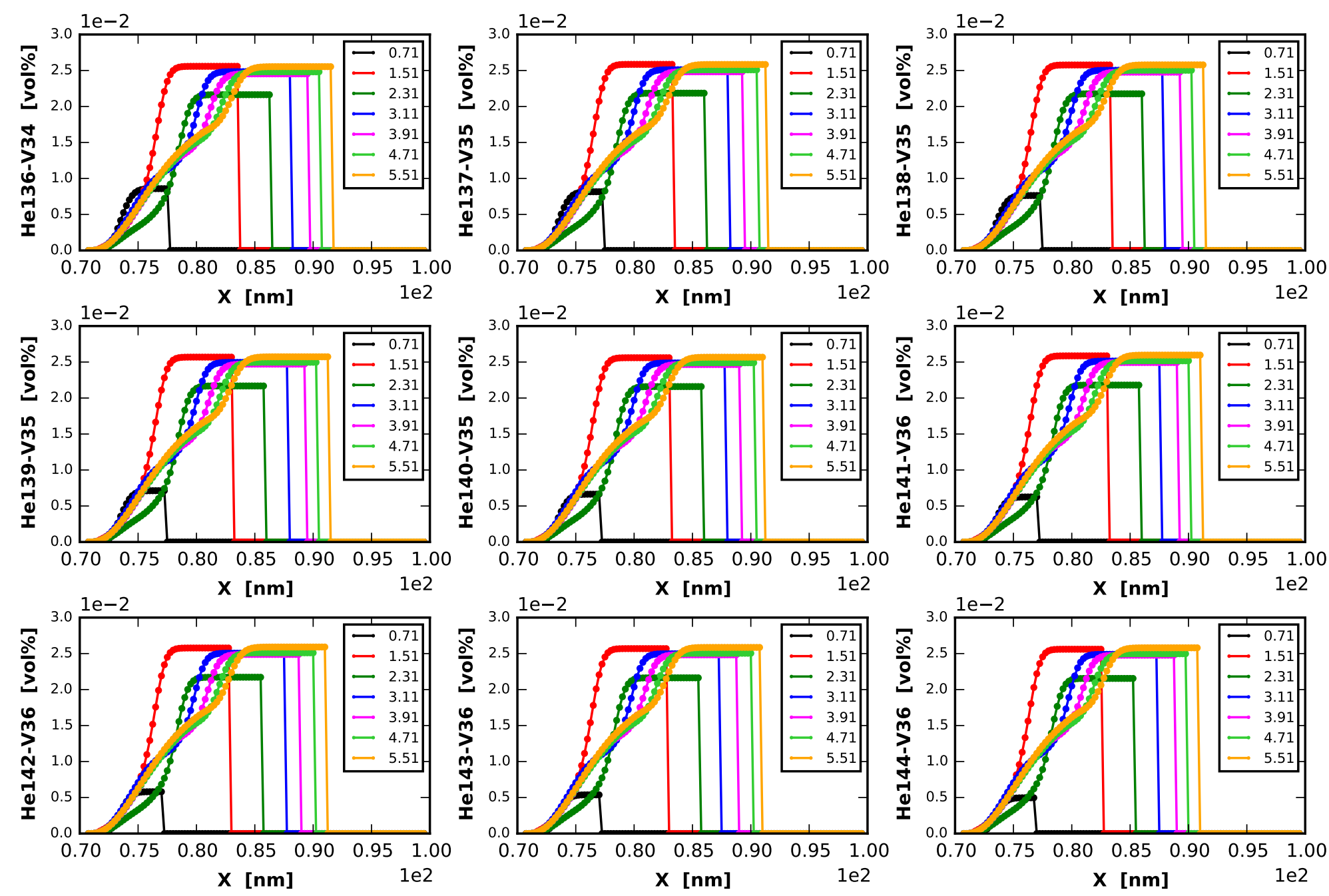


\section{Transient: [0.71, 1.51, 2.31, 3.11, 3.91, 4.71, 5.51] [us]}
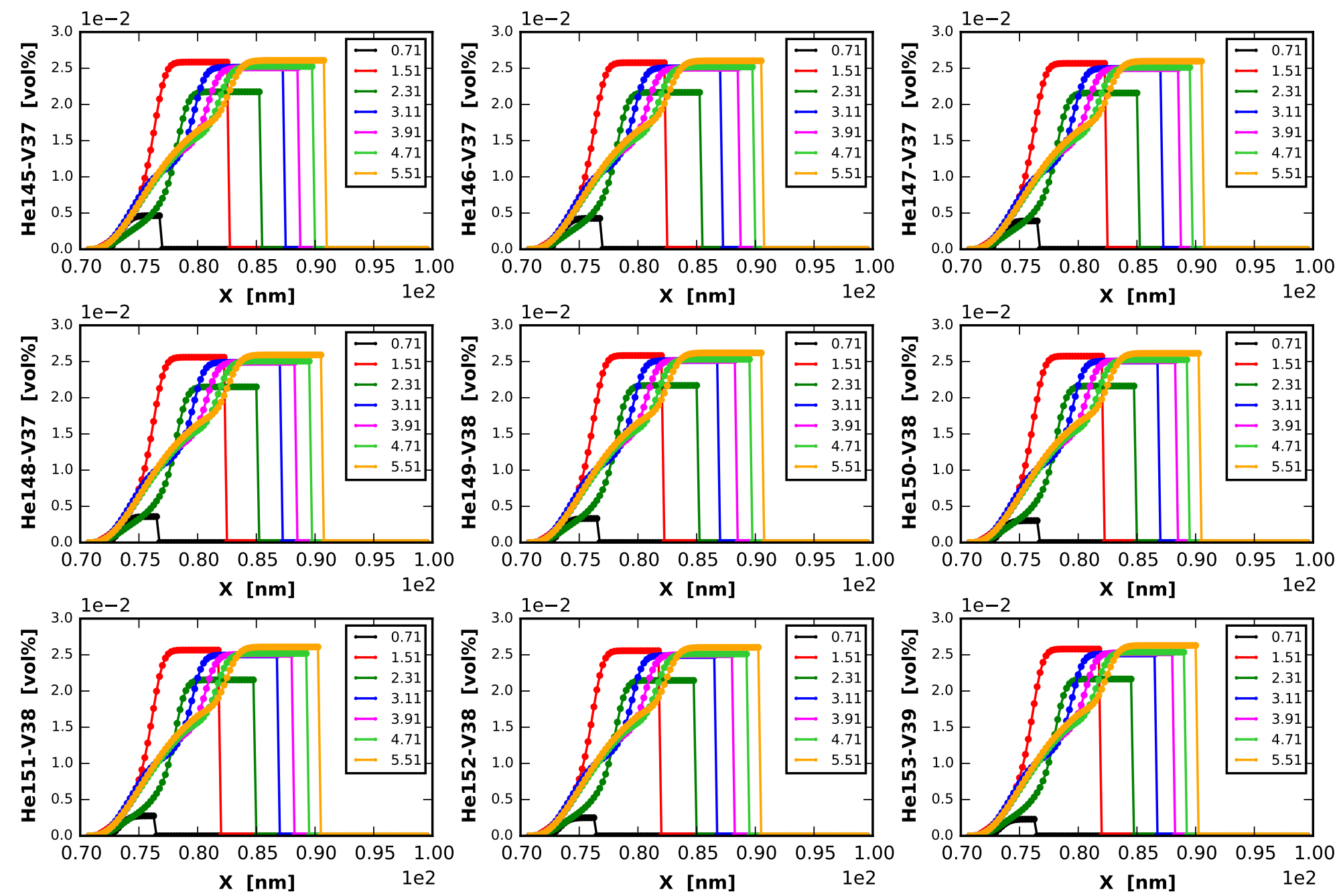


\section{Transient: [0.71, 1.51, 2.31, 3.11, 3.91, 4.71, 5.51] [us]}
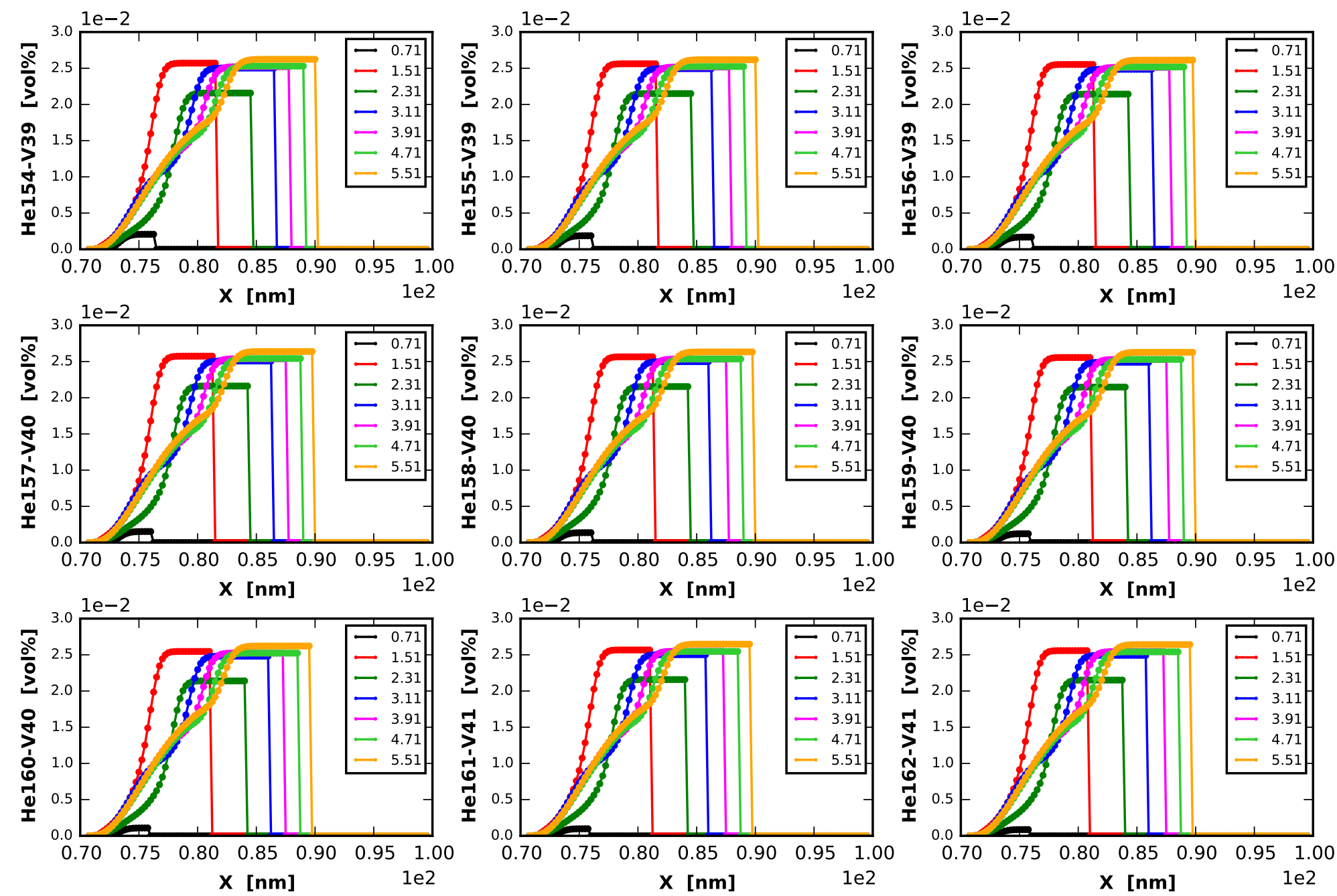


\section{Transient: [0.71, 1.51, 2.31, 3.11, 3.91, 4.71, 5.51] [us]}
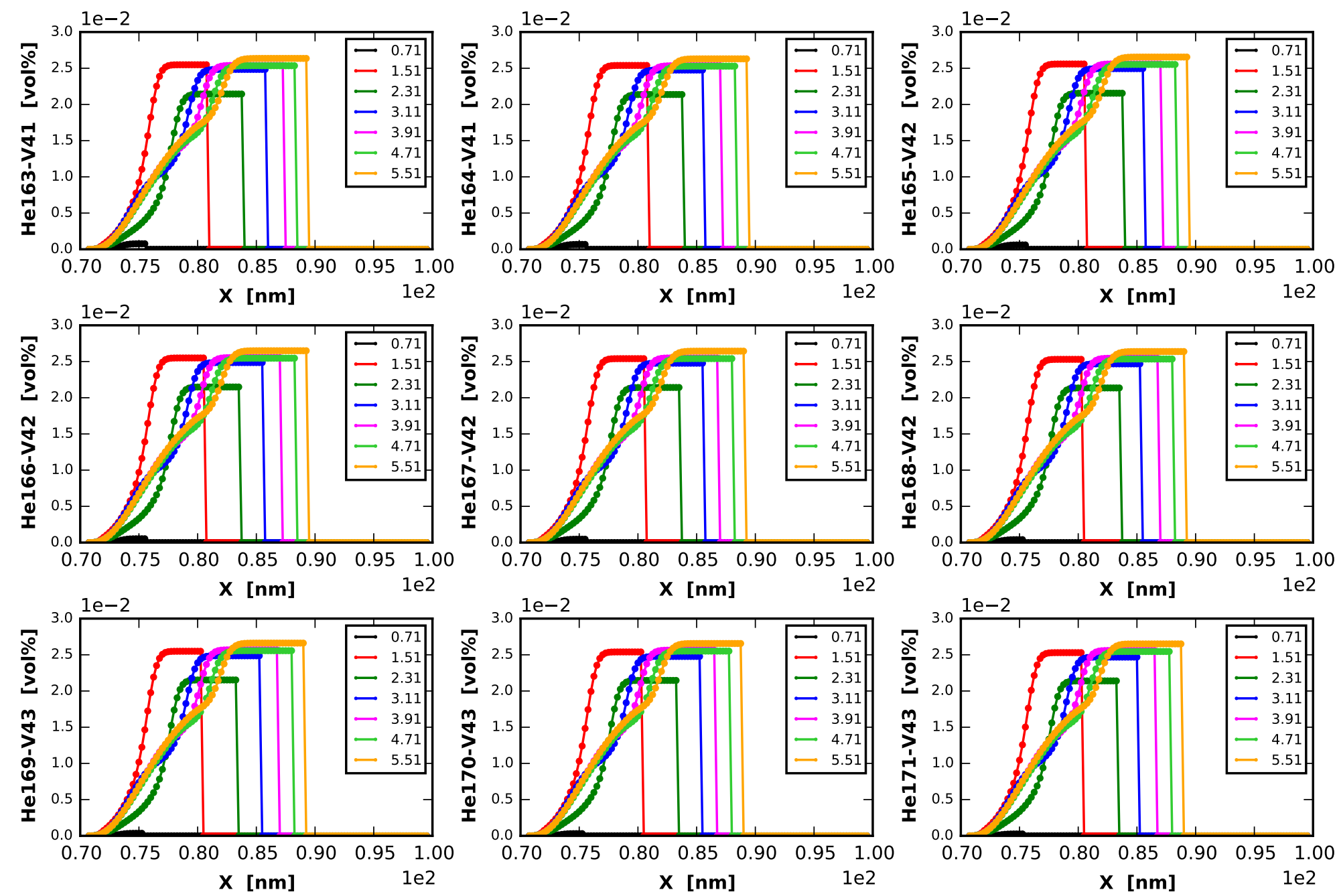


\section{Transient: [0.71, 1.51, 2.31, 3.11, 3.91, 4.71, 5.51] [us]}
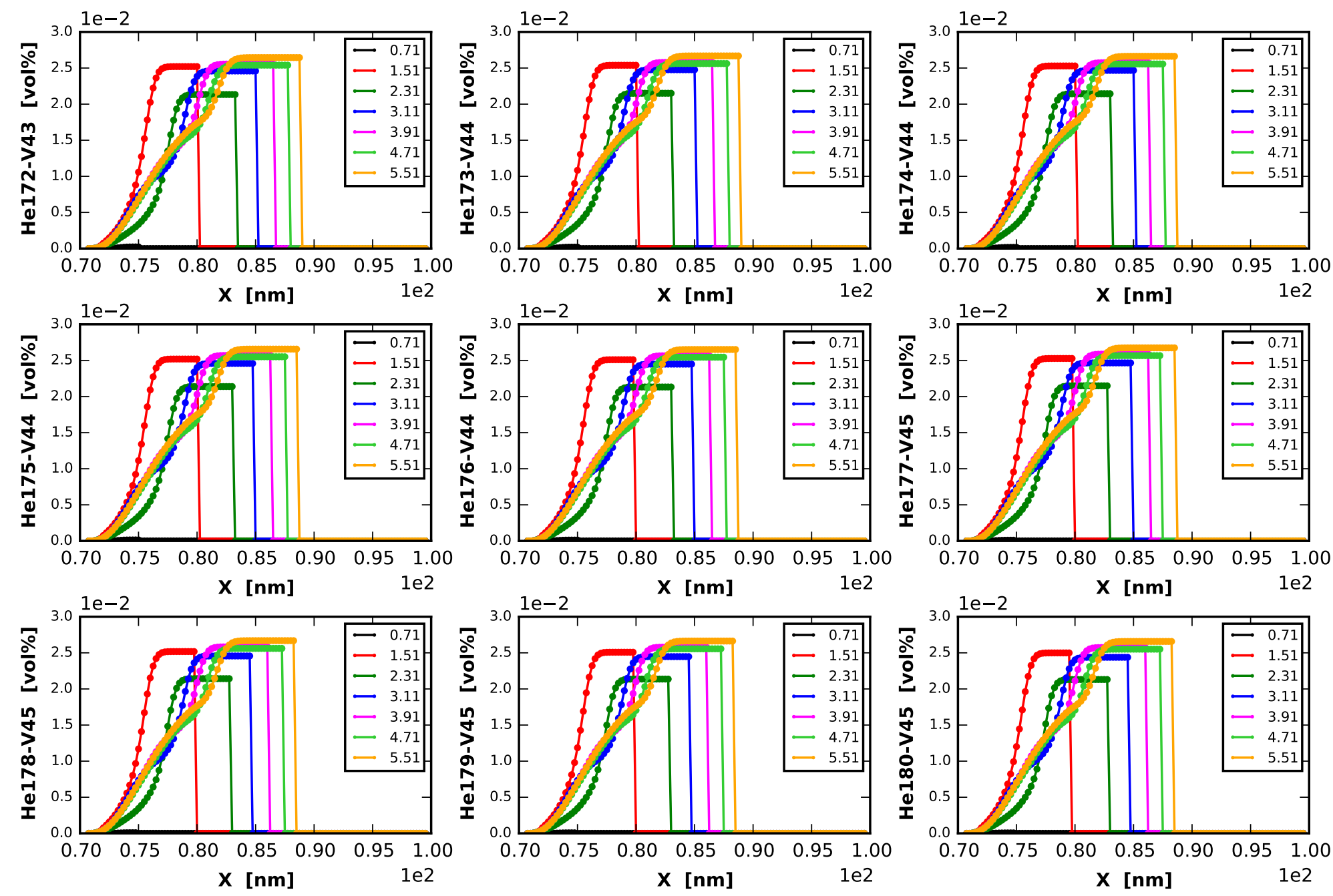


\section{Transient: [0.71, 1.51, 2.31, 3.11, 3.91, 4.71, 5.51] [us]}
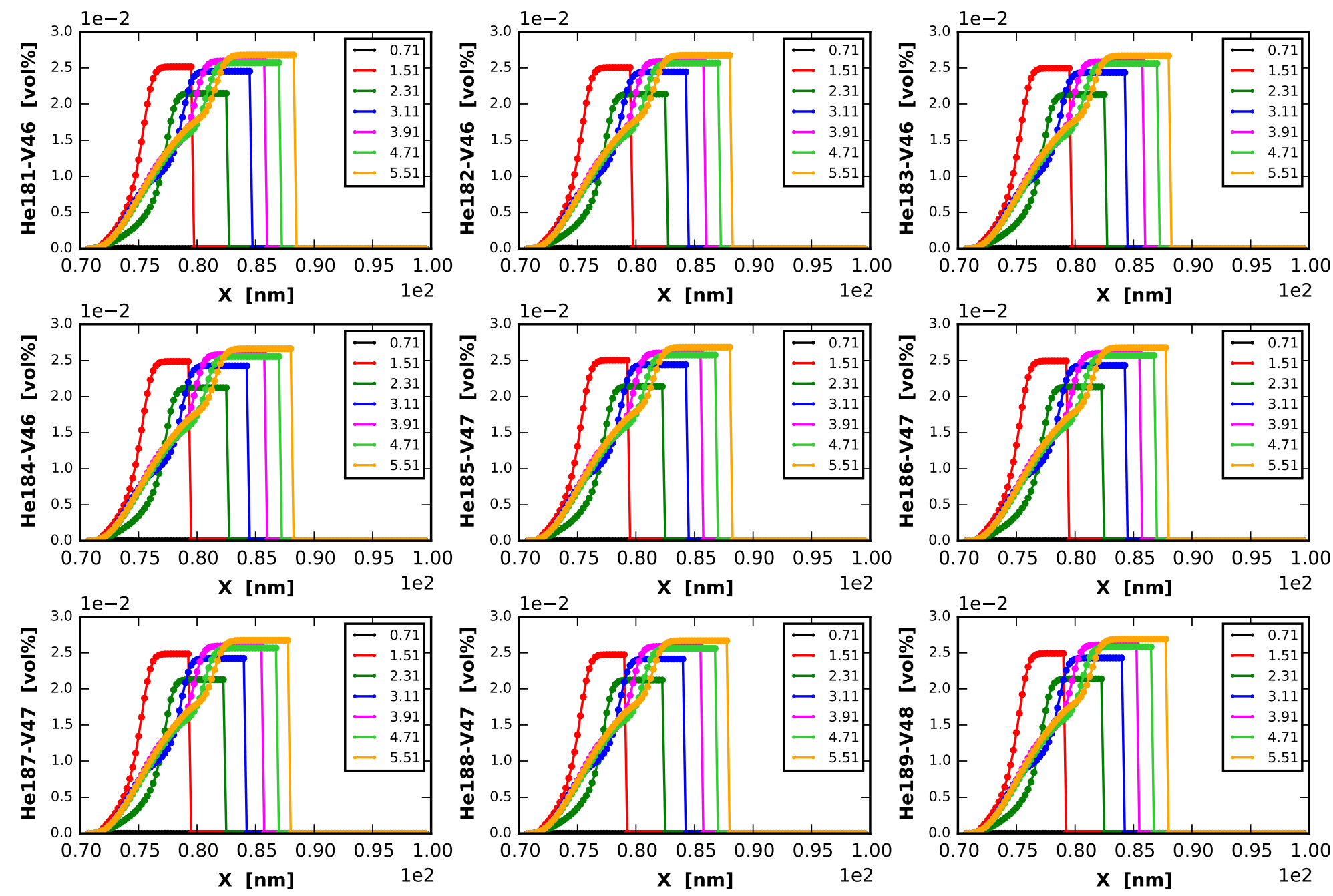
Transient: $[0.71,1.51,2.31,3.11,3.91,4.71,5.51]$ [us]
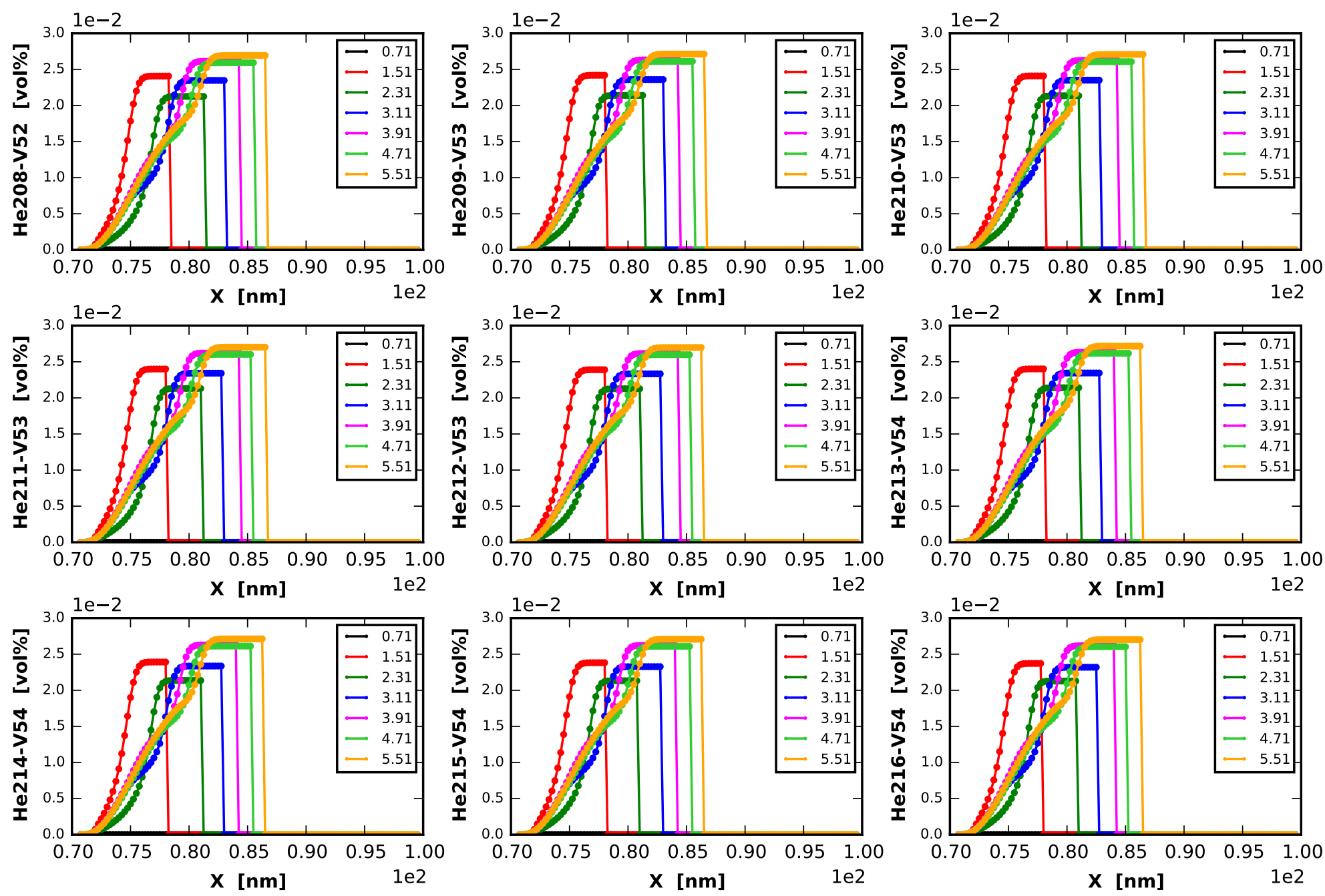
Transient: $[0.71,1.51,2.31,3.11,3.91,4.71,5.51]$ [us]
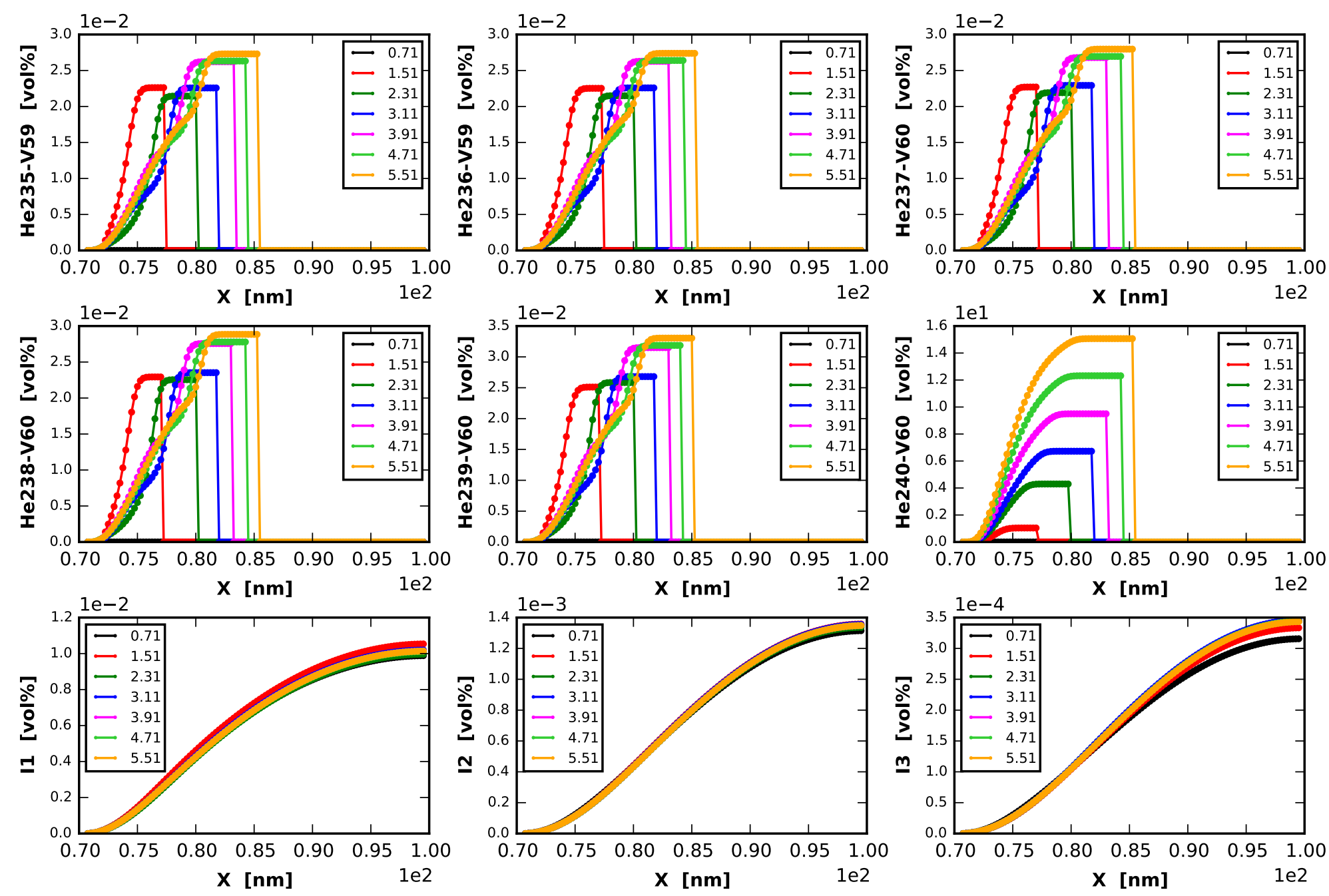


\section{Transient: [0.71, 1.51, 2.31, 3.11, 3.91, 4.71, 5.51] [us]}
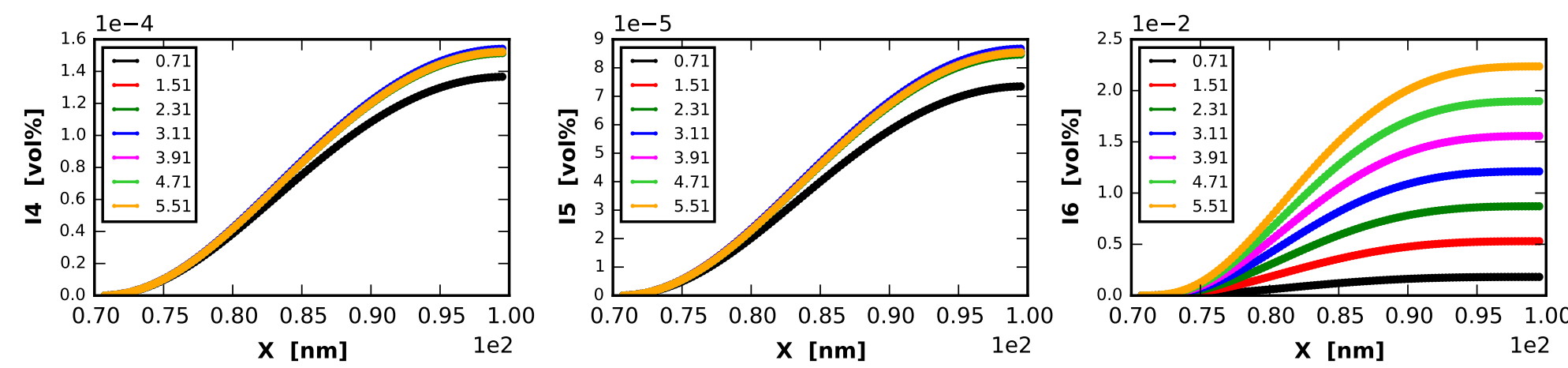
Bubble Nucleation, Growth and Coalescence ...

\section{B Appendix: Case $\mathbf{H e}_{1000}-\mathrm{V}_{250}$}

\section{B.1 Volume fraction plots}


Transient: [0.71, 1.51, 2.31, 3.11, 3.91, 4.71, 5.51] [us]
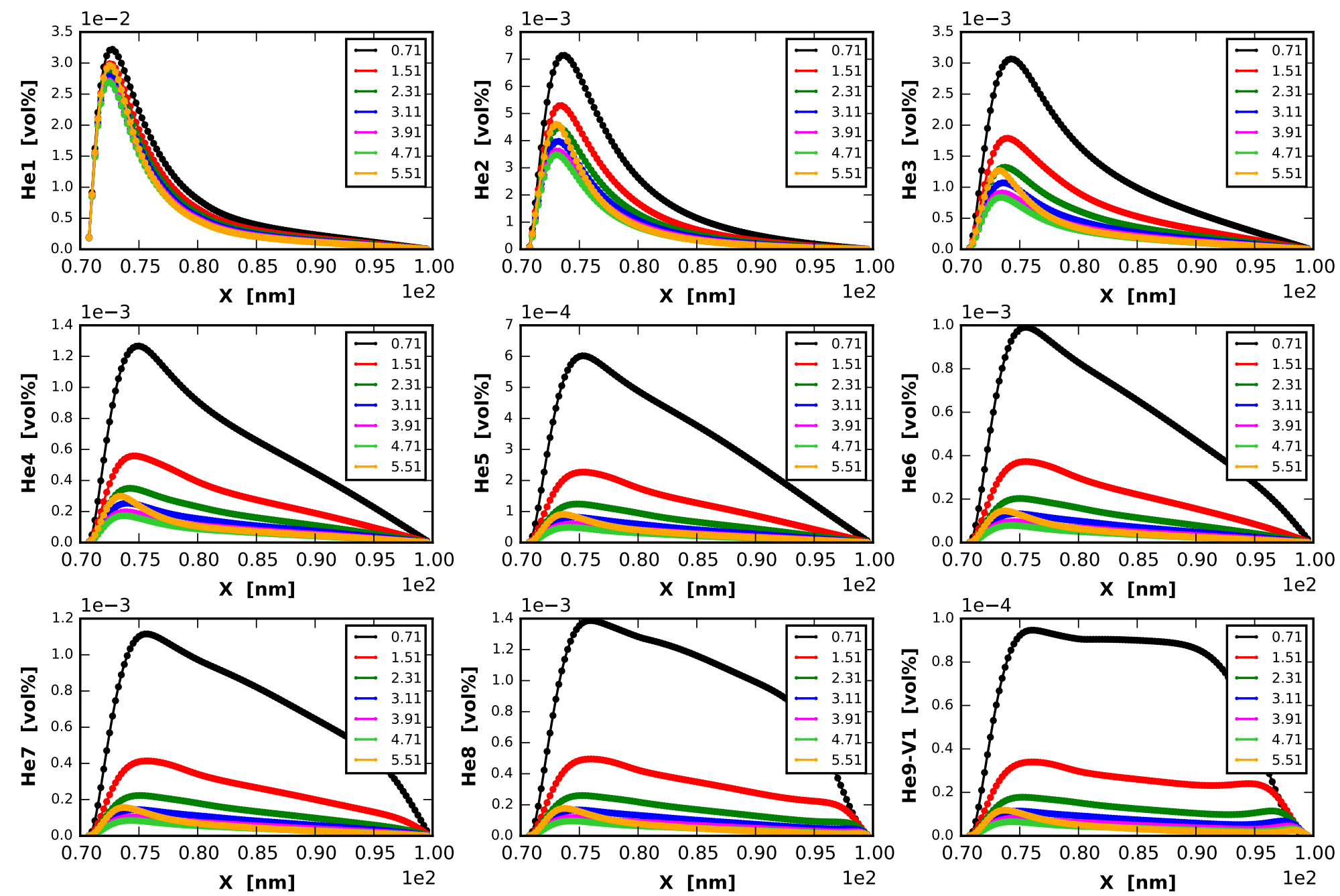
Transient: [0.71, 1.51, 2.31, 3.11, 3.91, 4.71, 5.51] [us]
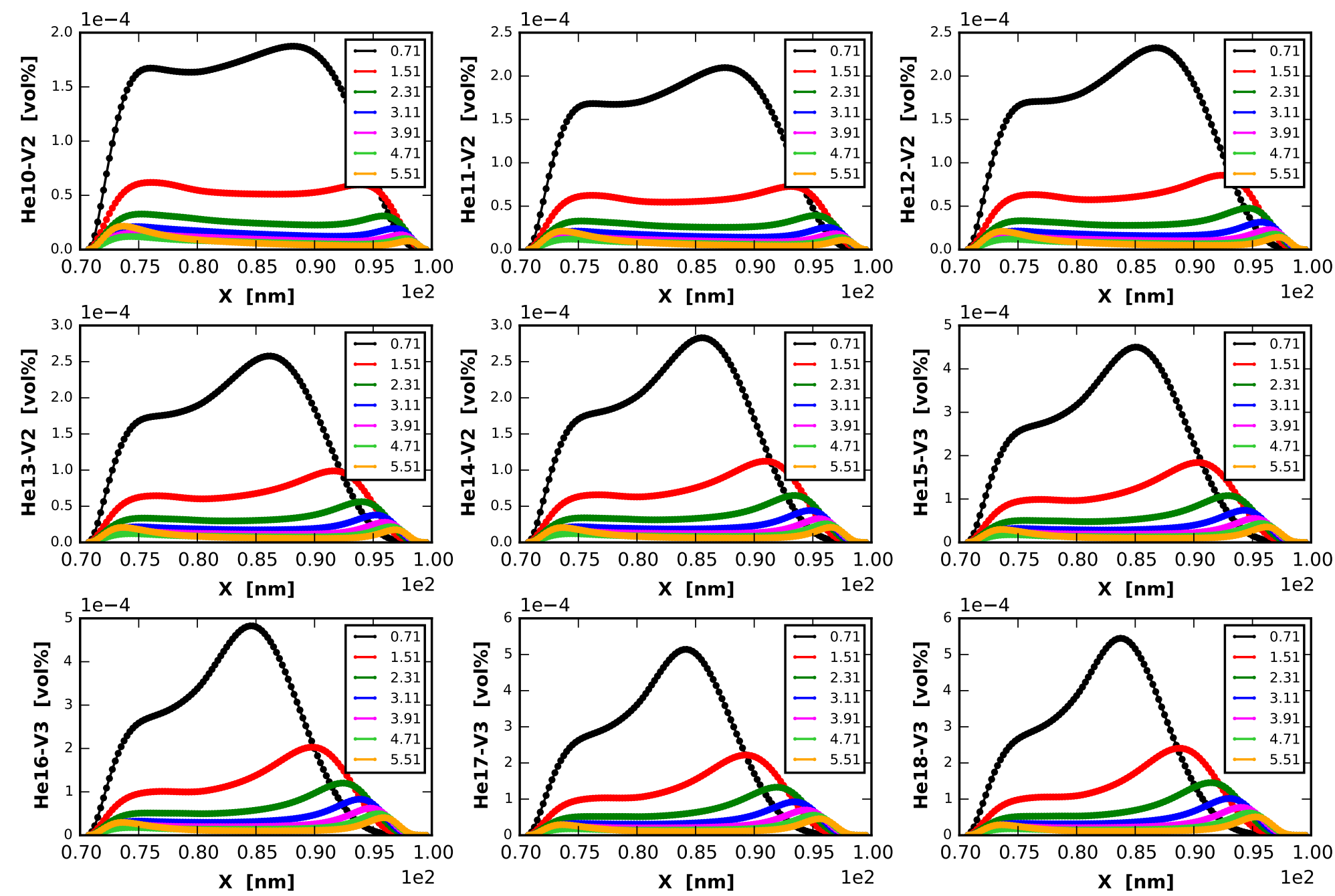
Transient: [0.71, 1.51, 2.31, 3.11, 3.91, 4.71, 5.51] [us]
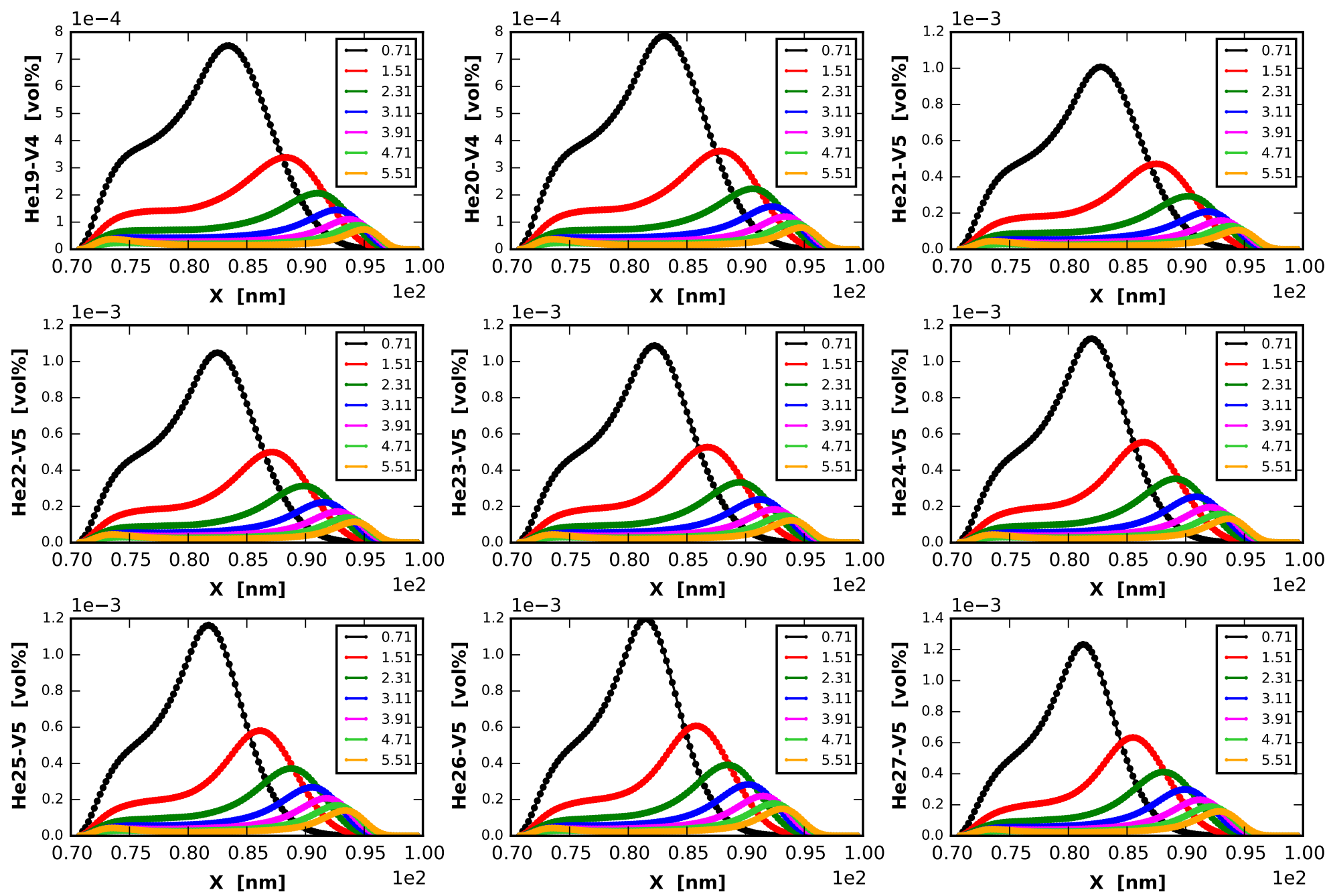
Transient: $[0.71,1.51,2.31,3.11,3.91,4.71,5.51]$ [us]
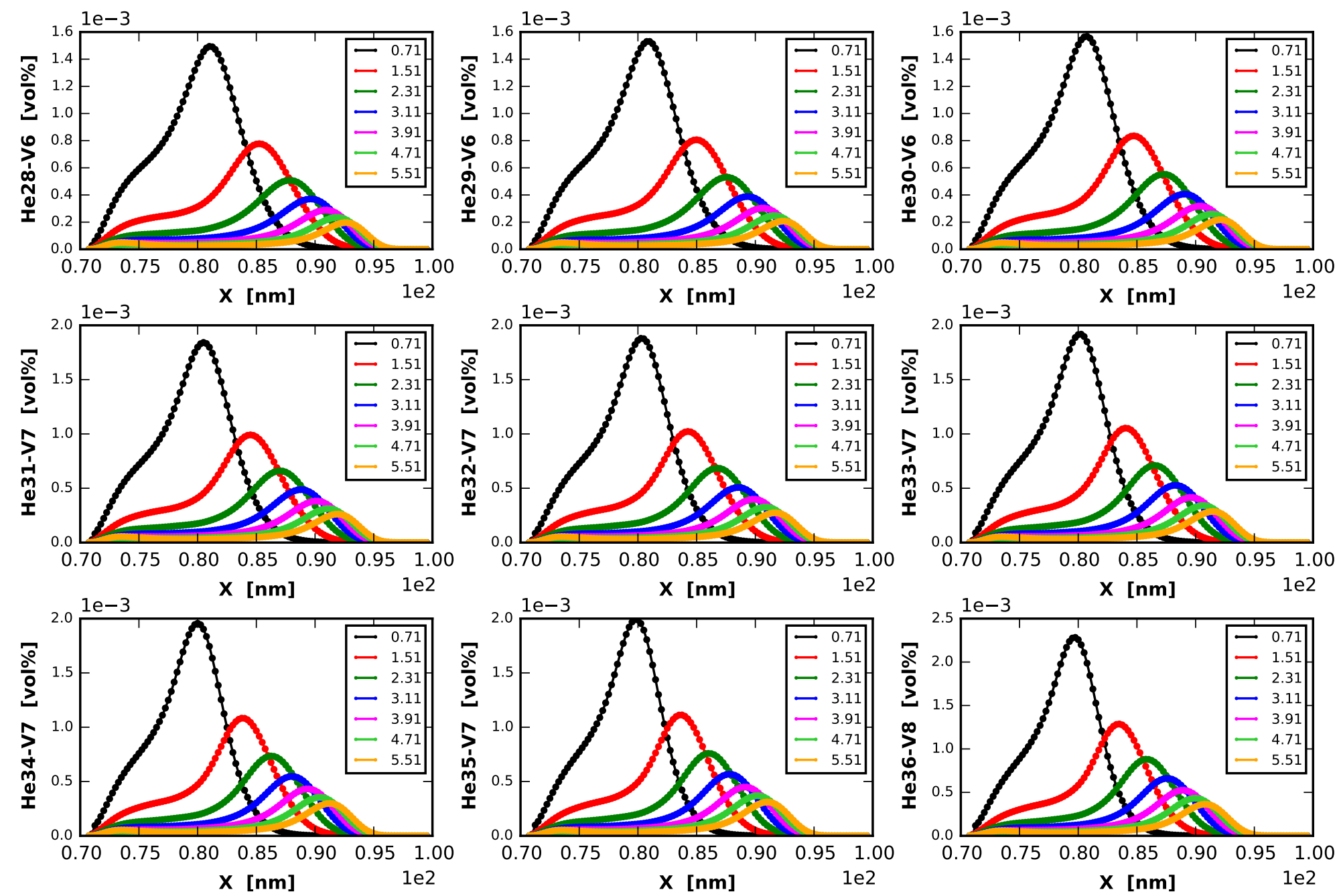
Transient: $[0.71,1.51,2.31,3.11,3.91,4.71,5.51]$ [us]
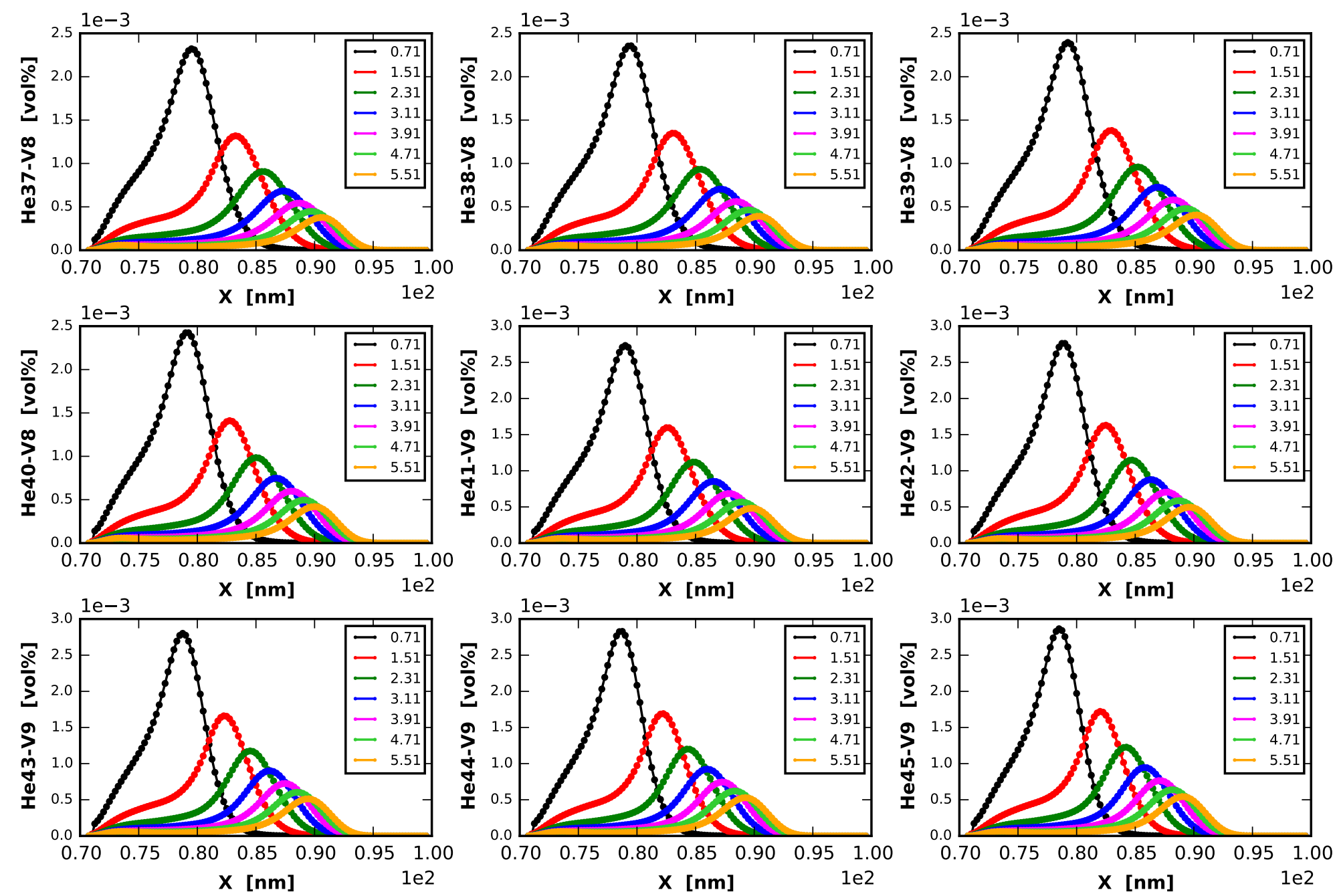



\section{Transient: [0.71, 1.51, 2.31, 3.11, 3.91, 4.71, 5.51] [us]}
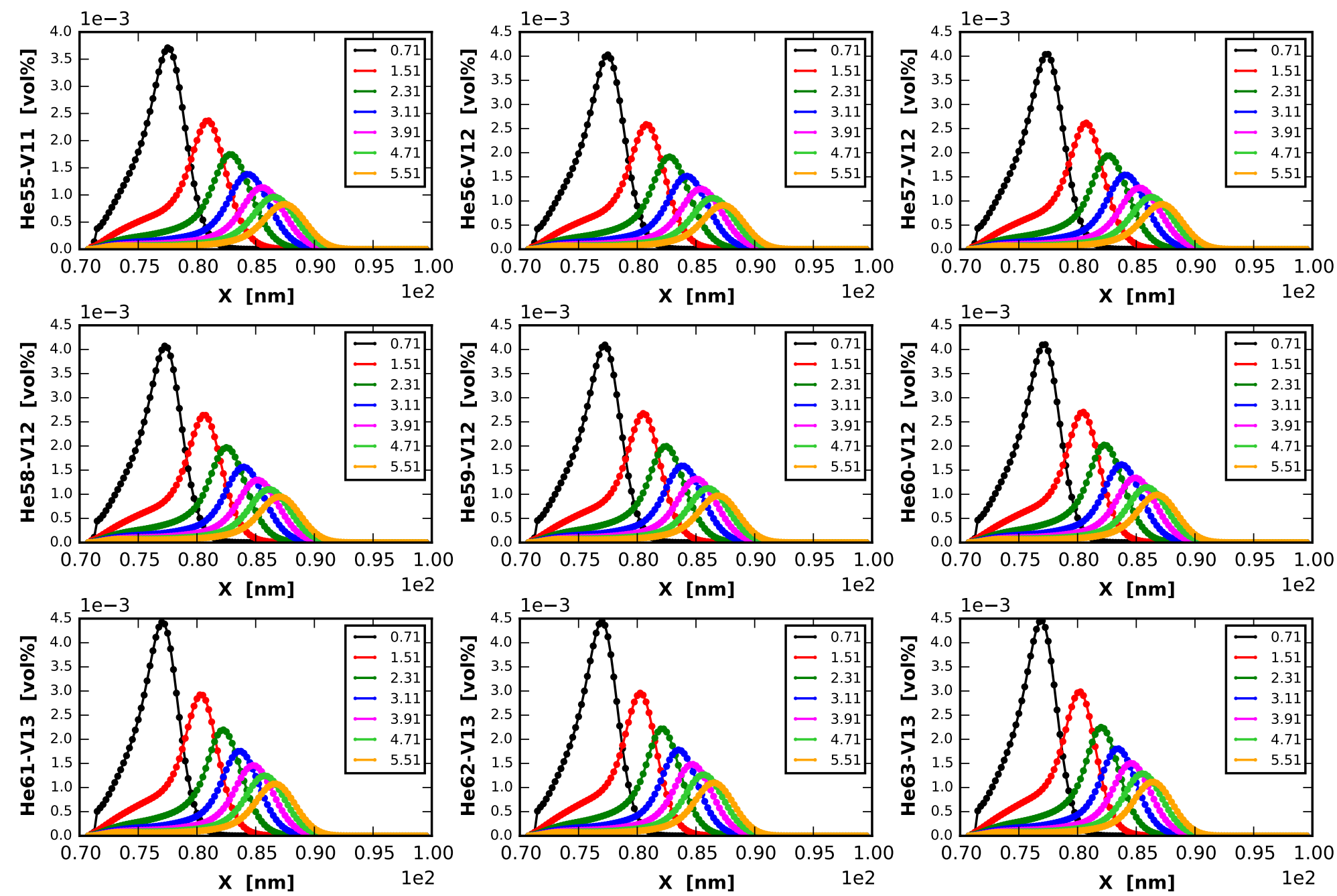
Transient: $[0.71,1.51,2.31,3.11,3.91,4.71,5.51]$ [us]
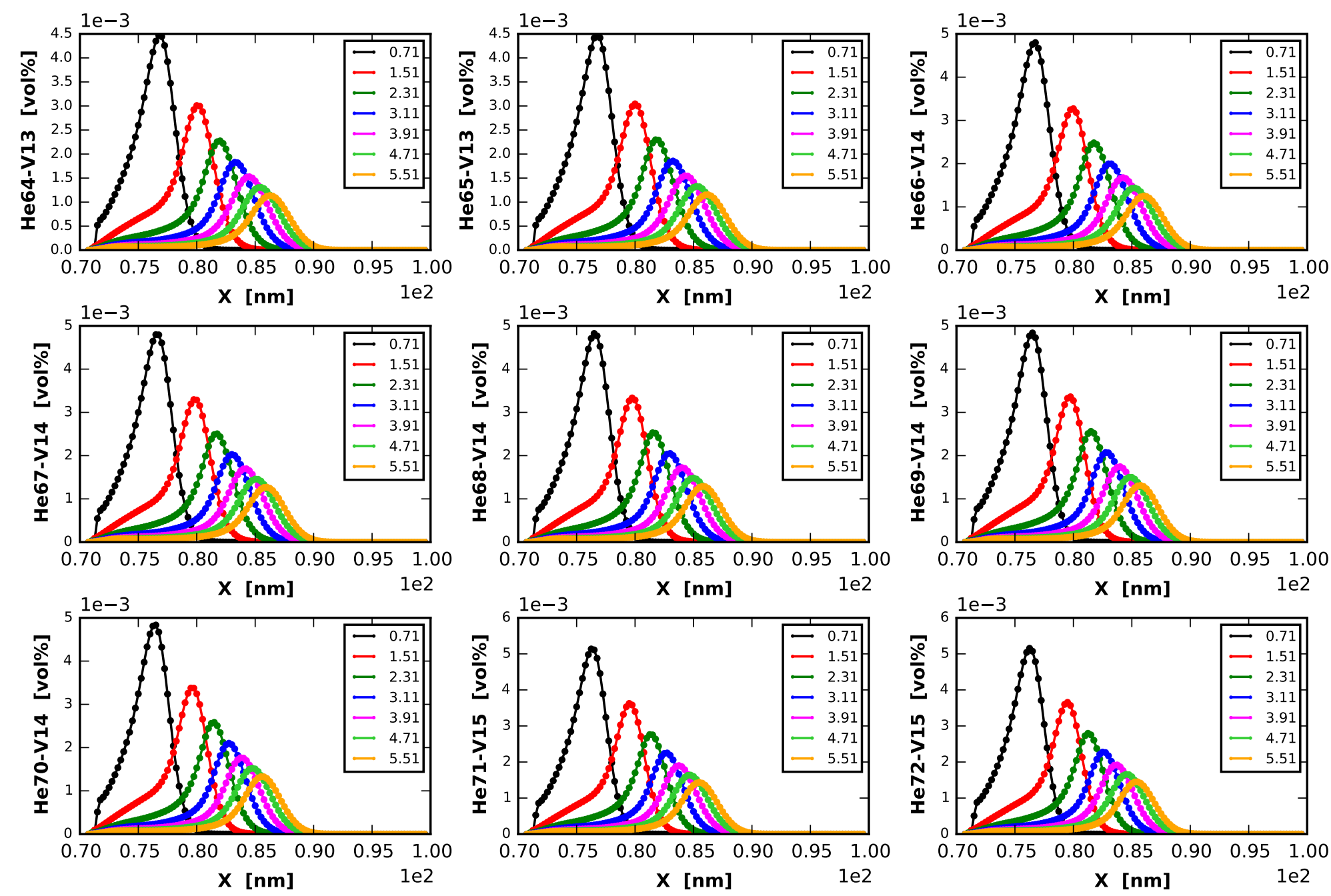
Transient: $[0.71,1.51,2.31,3.11,3.91,4.71,5.51]$ [us]
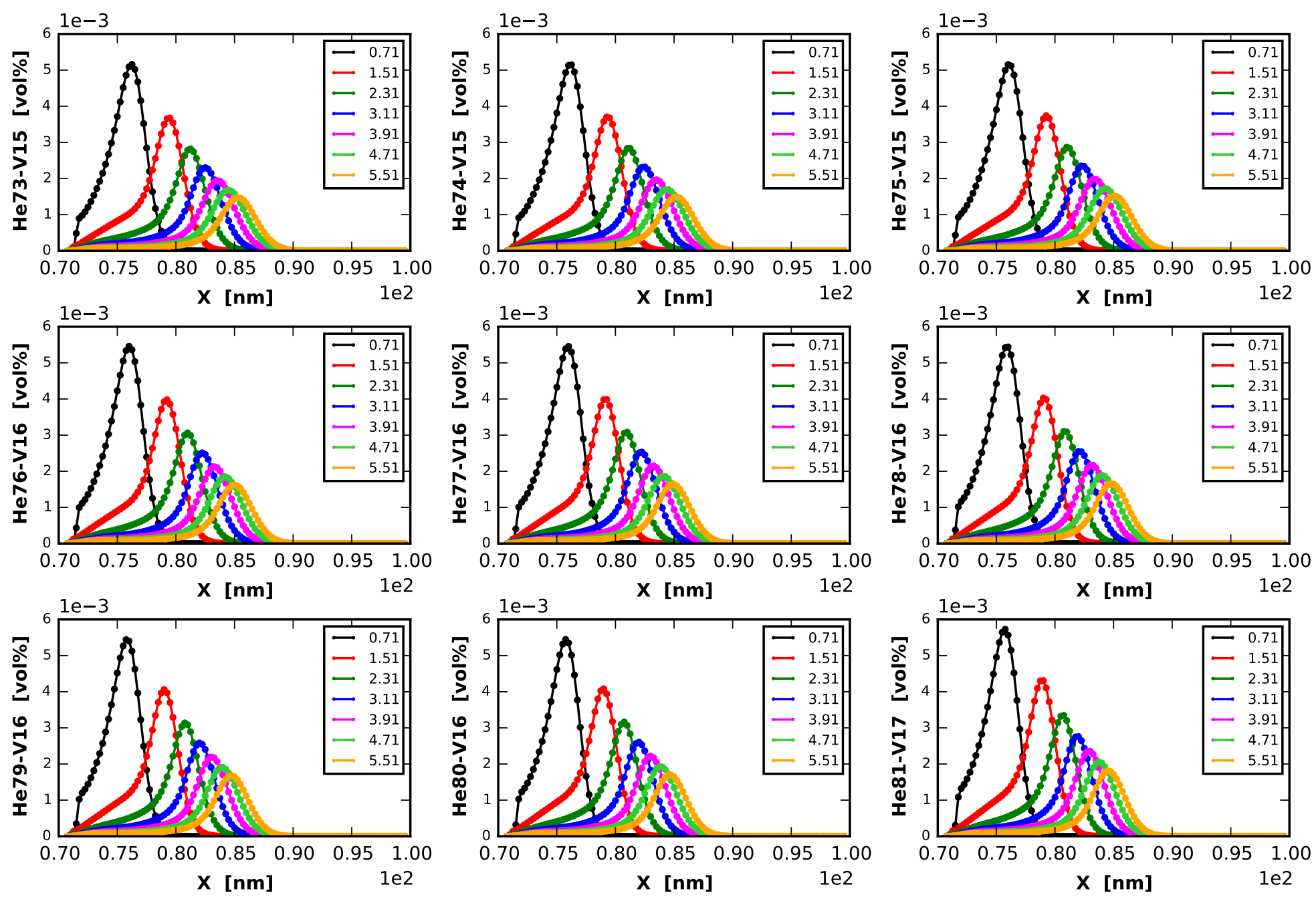
Transient: $[0.71,1.51,2.31,3.11,3.91,4.71,5.51]$ [us]
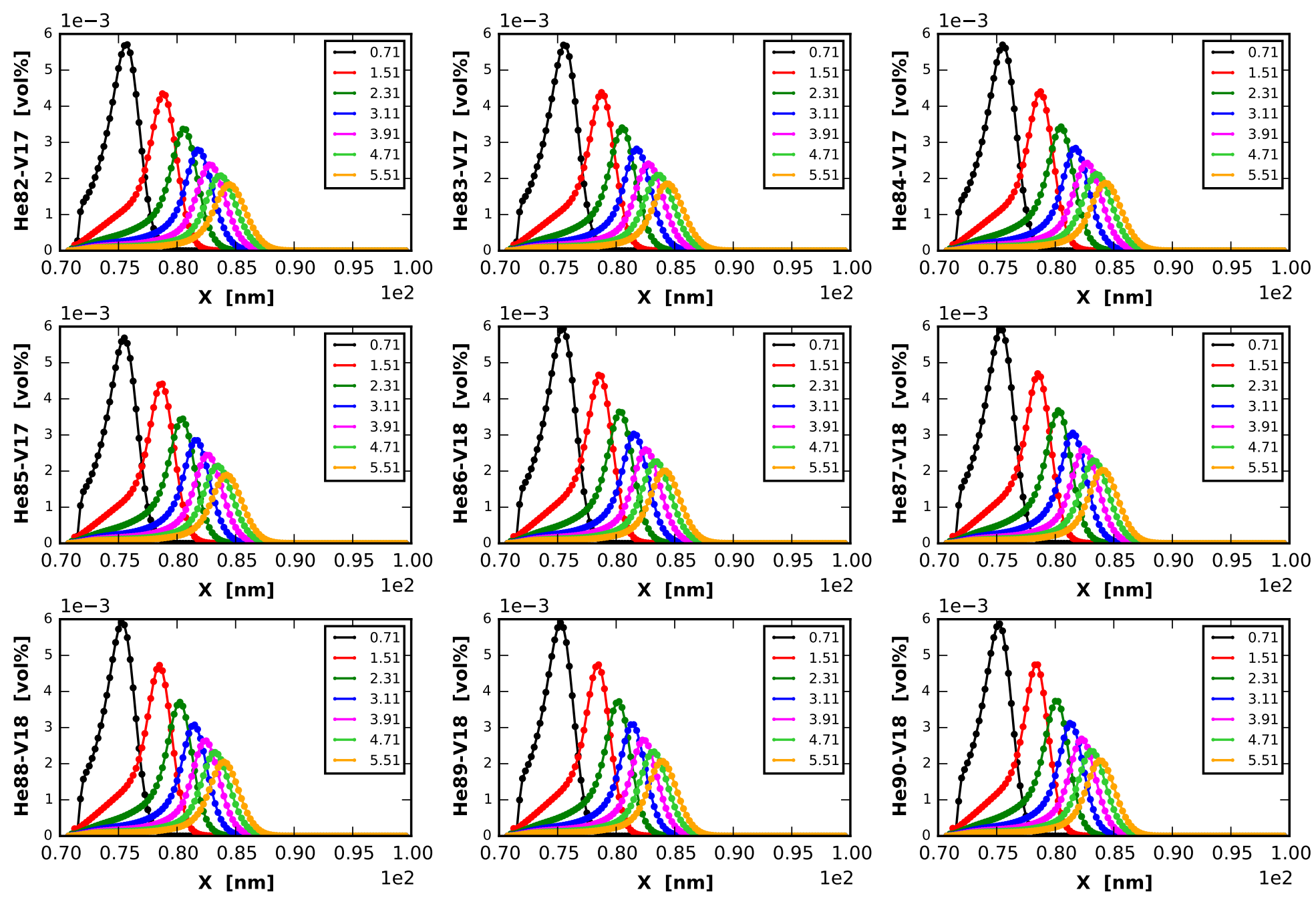
Transient: $[0.71,1.51,2.31,3.11,3.91,4.71,5.51]$ [us]
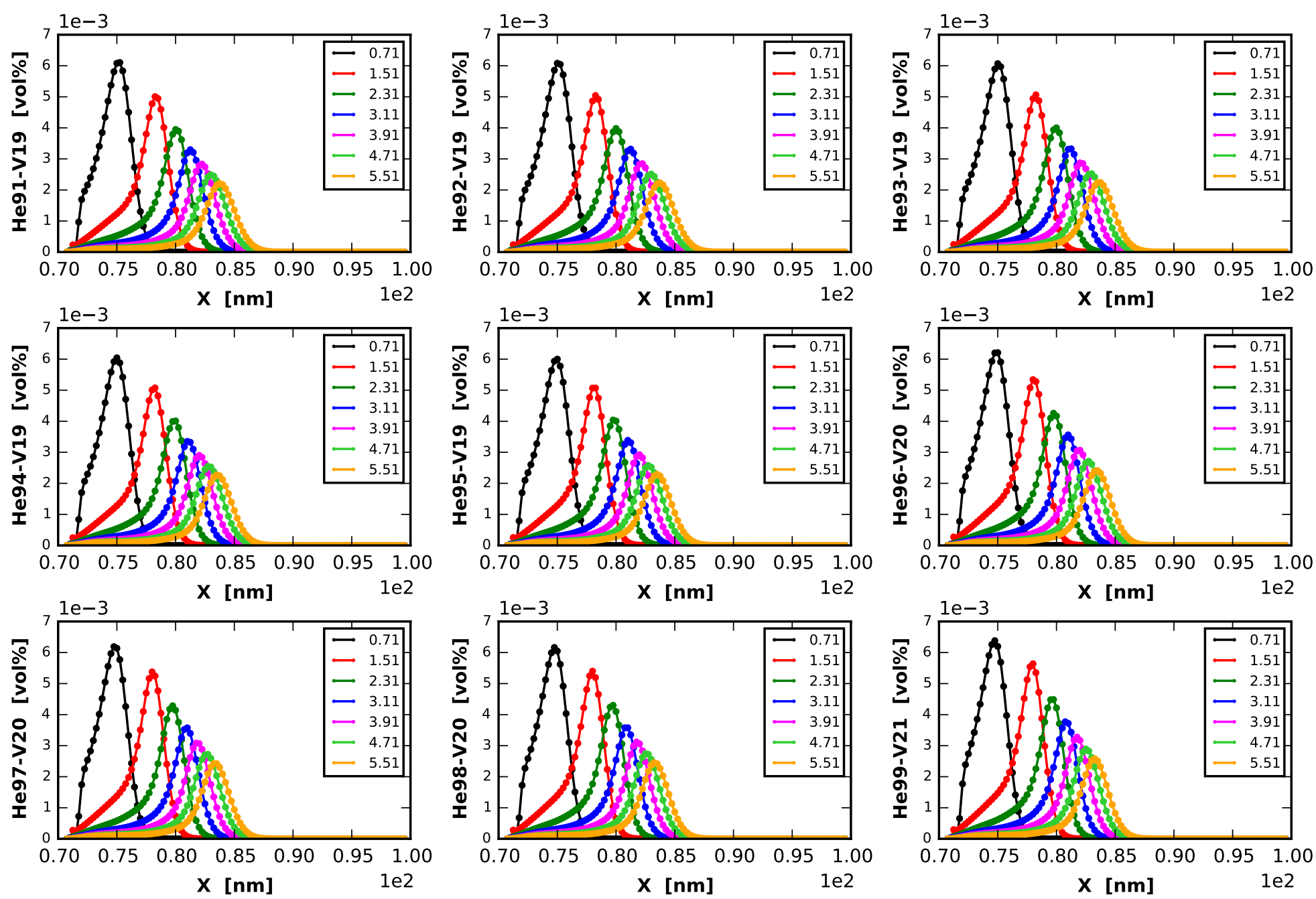
Transient: $[0.71,1.51,2.31,3.11,3.91,4.71,5.51]$ [us]
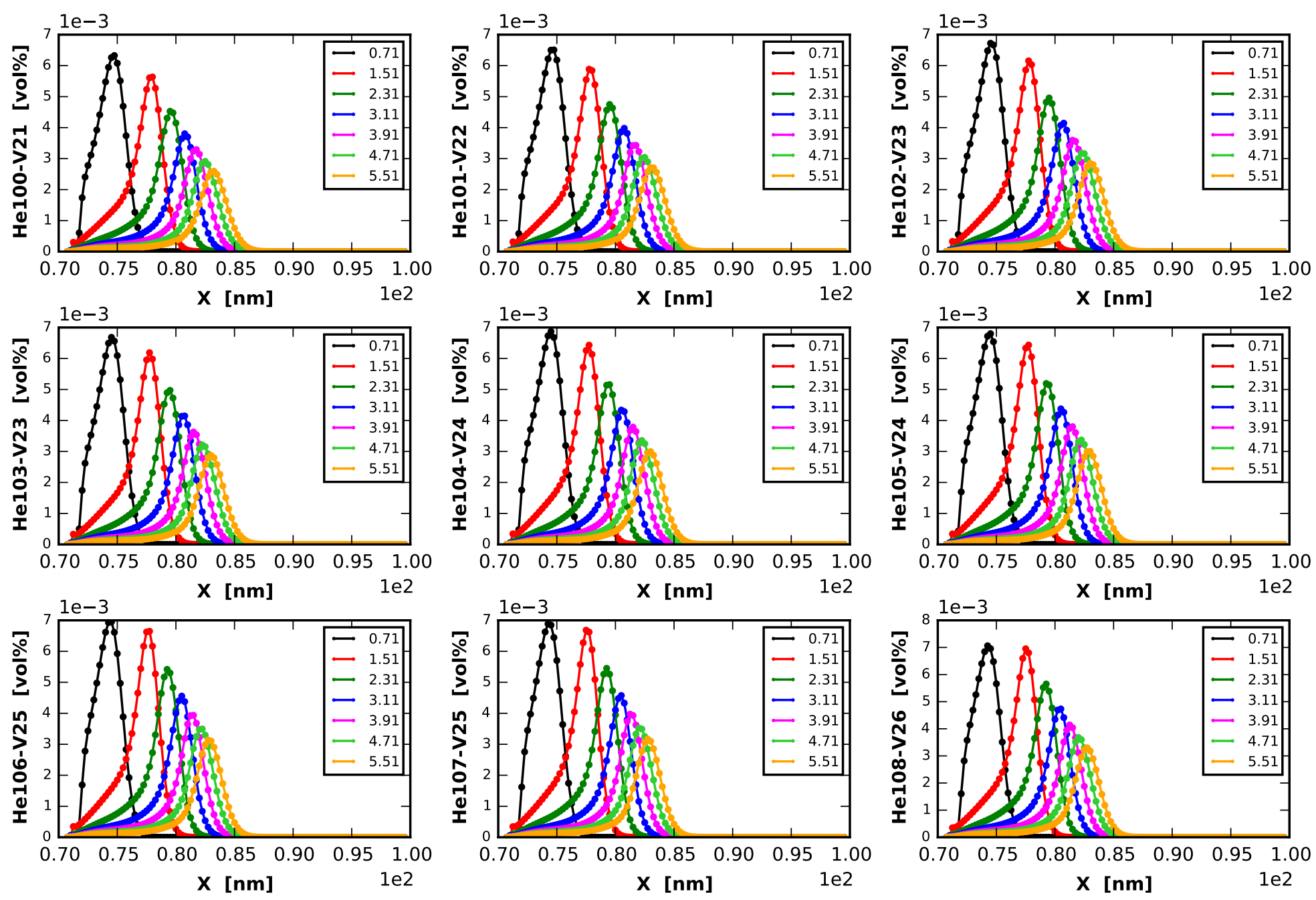
Transient: $[0.71,1.51,2.31,3.11,3.91,4.71,5.51]$ [us]
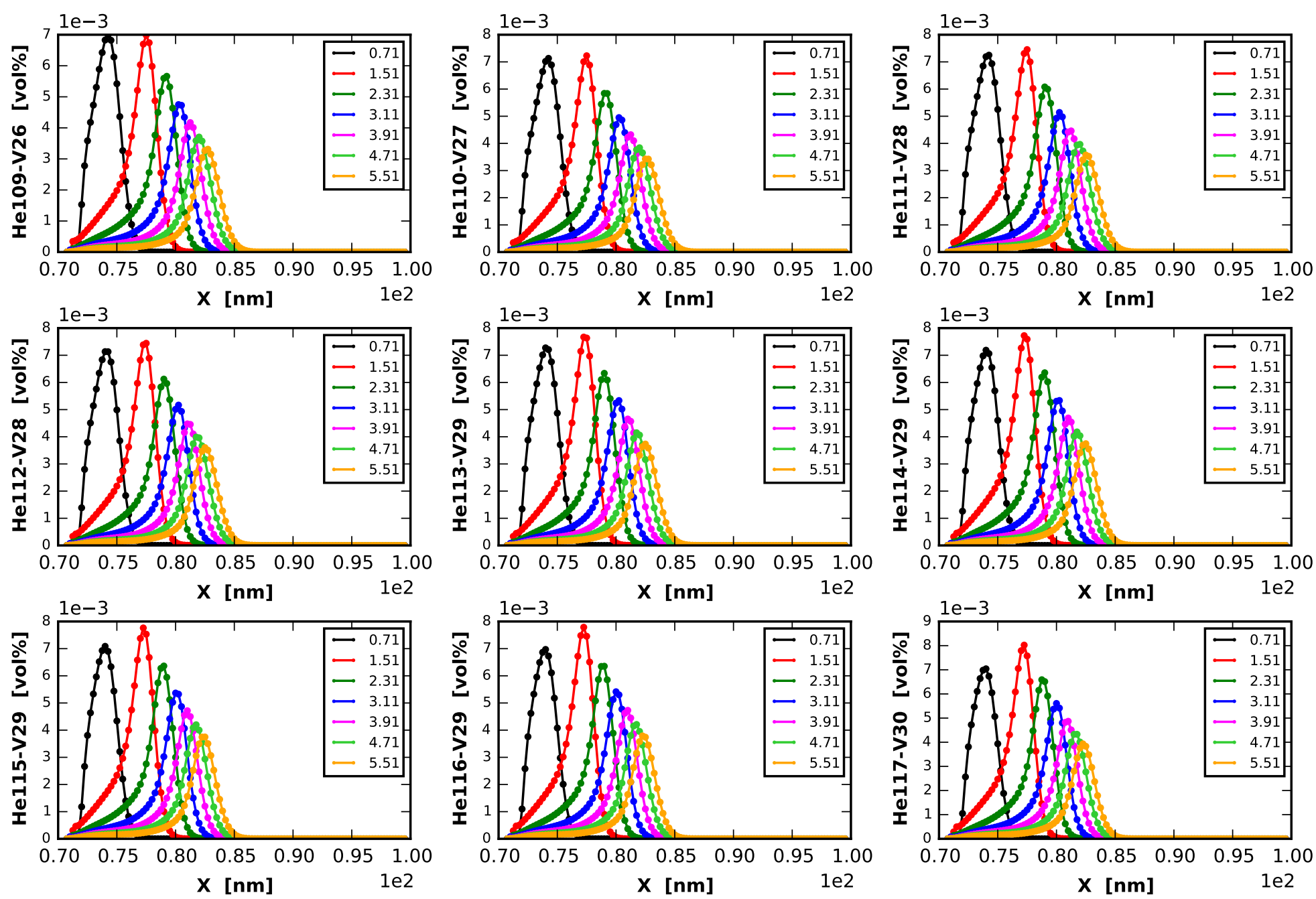
Transient: $[0.71,1.51,2.31,3.11,3.91,4.71,5.51]$ [us]
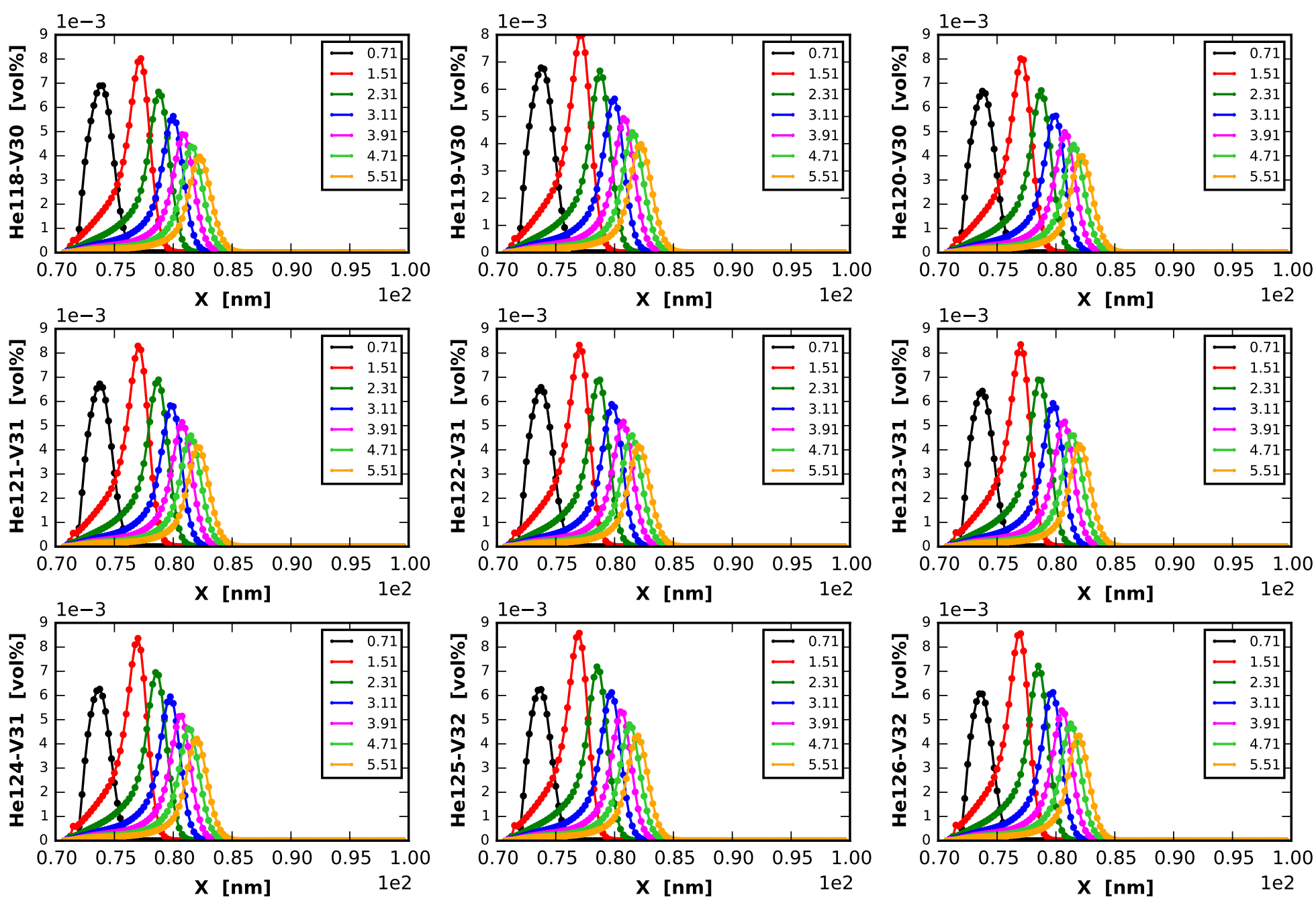
Transient: $[0.71,1.51,2.31,3.11,3.91,4.71,5.51]$ [us]
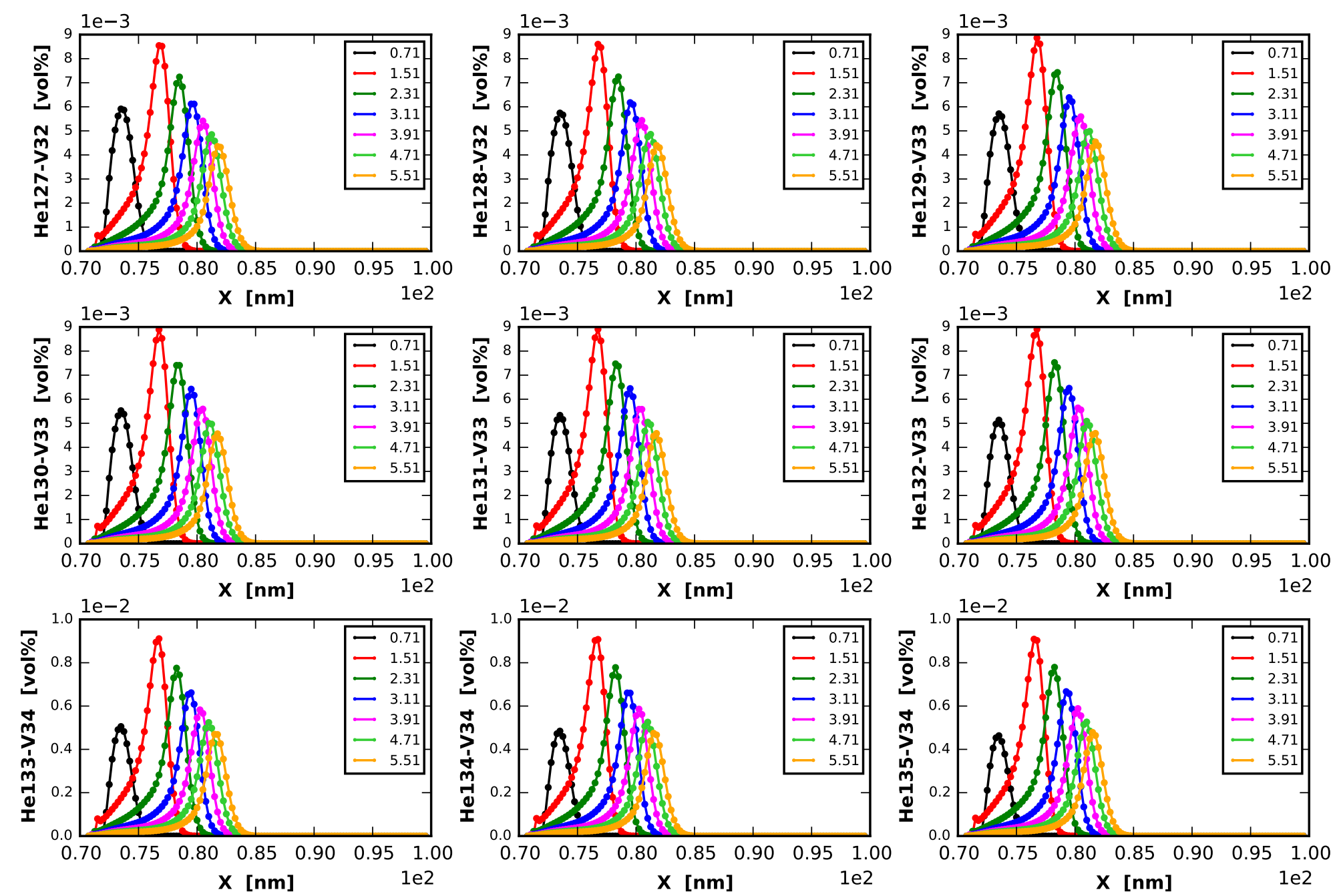
Transient: $[0.71,1.51,2.31,3.11,3.91,4.71,5.51]$ [us]
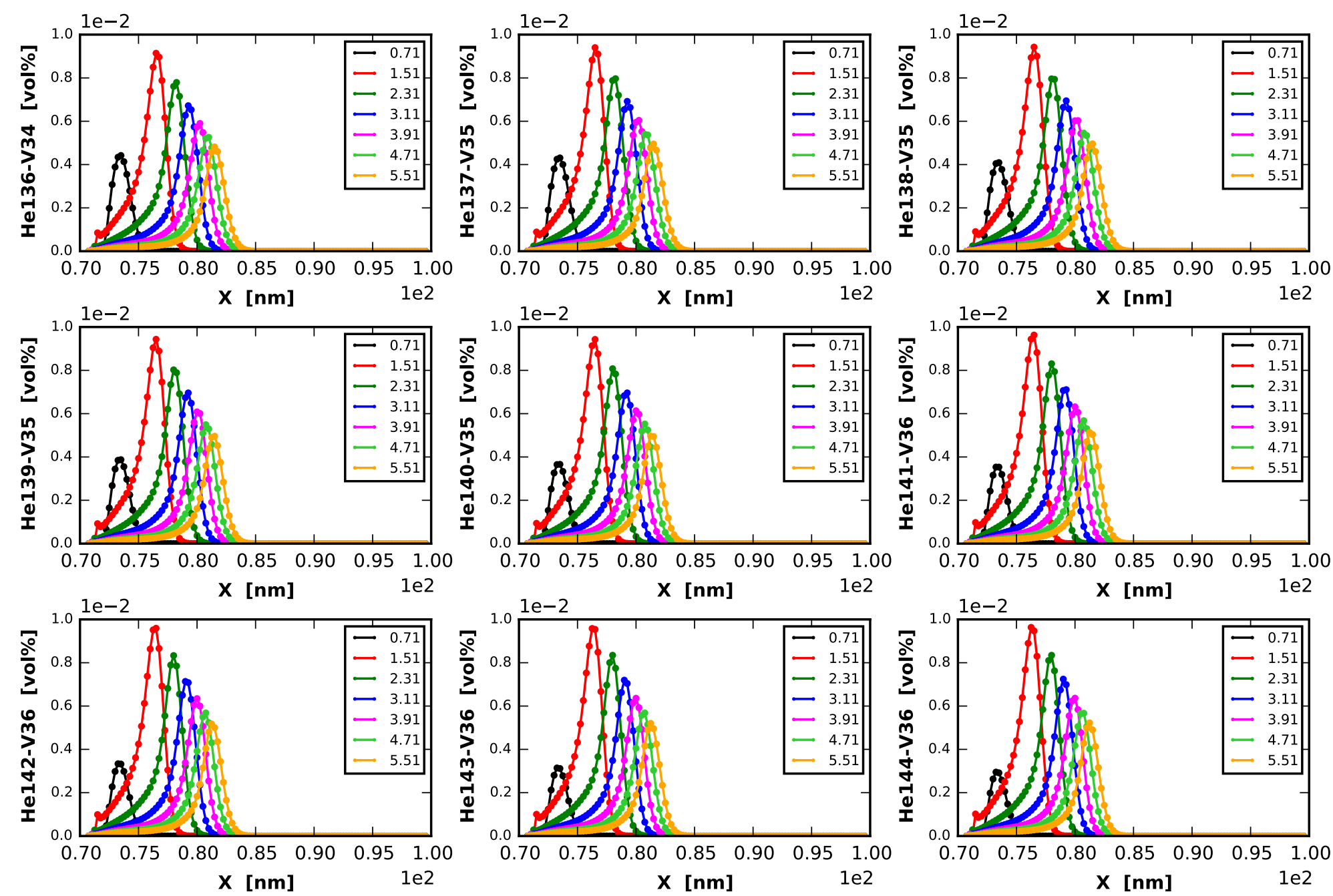
Transient: $[0.71,1.51,2.31,3.11,3.91,4.71,5.51]$ [us]
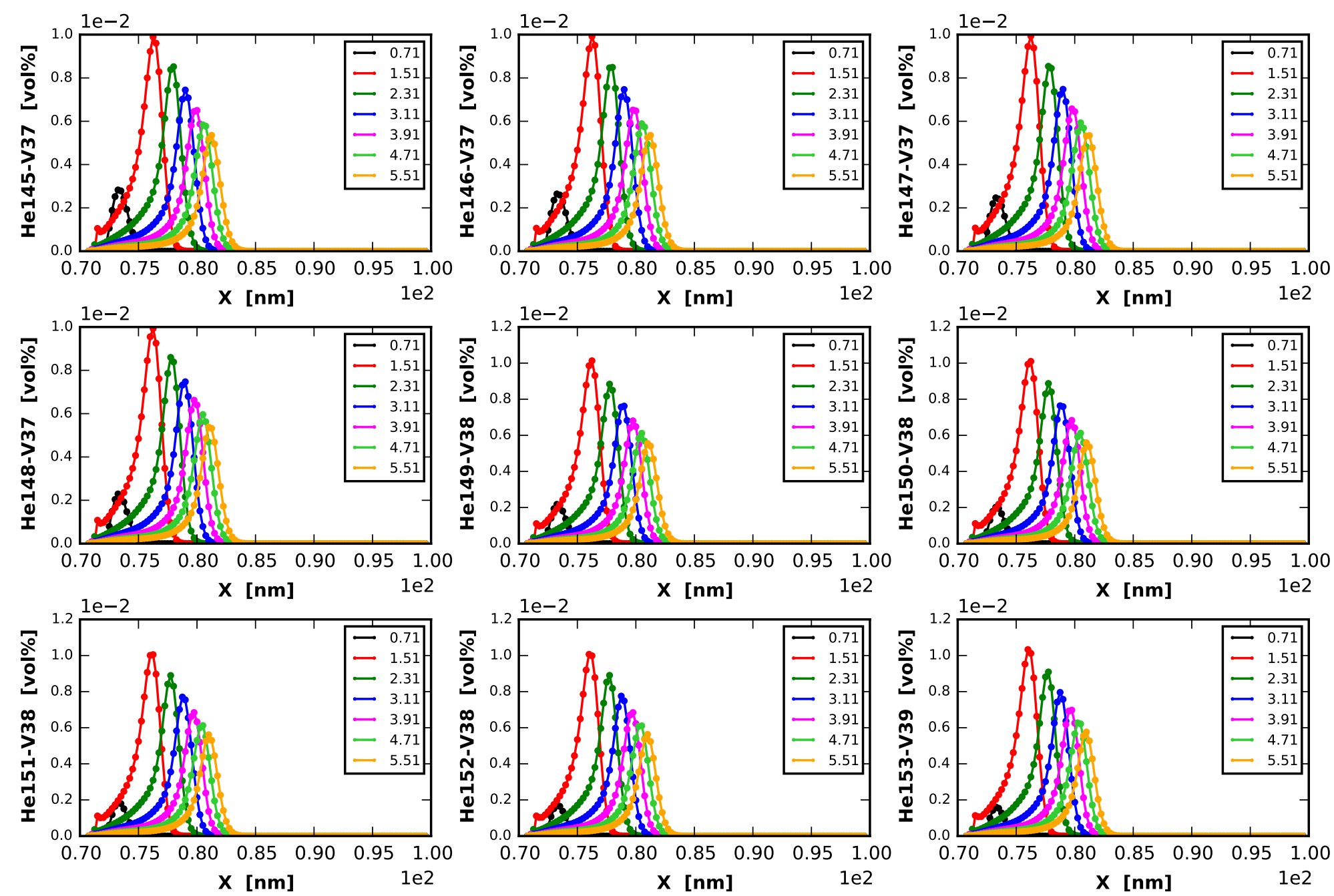
Transient: [0.71, 1.51, 2.31, 3.11, 3.91, 4.71, 5.51] [us]
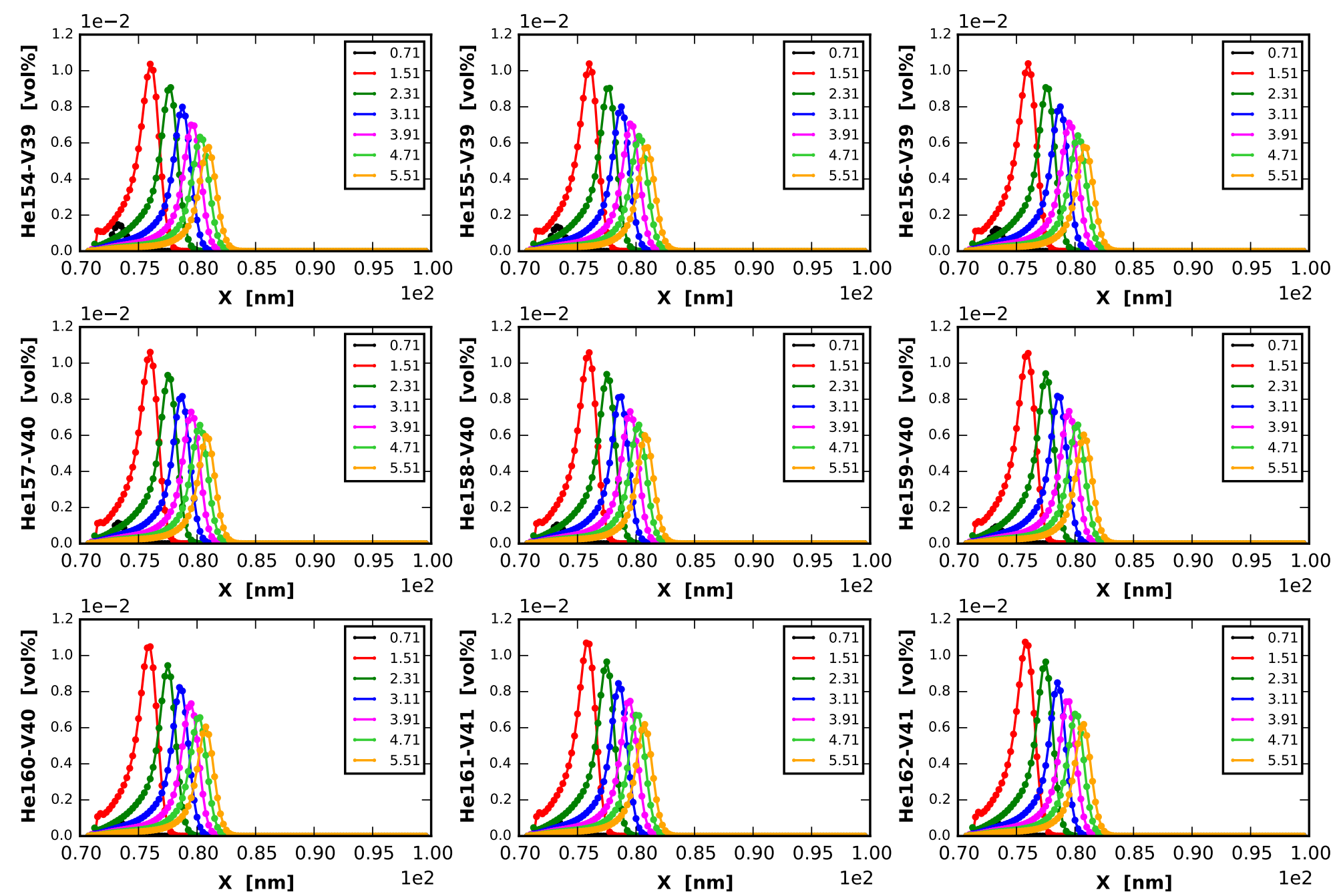
Transient: [0.71, 1.51, 2.31, 3.11, 3.91, 4.71, 5.51] [us]
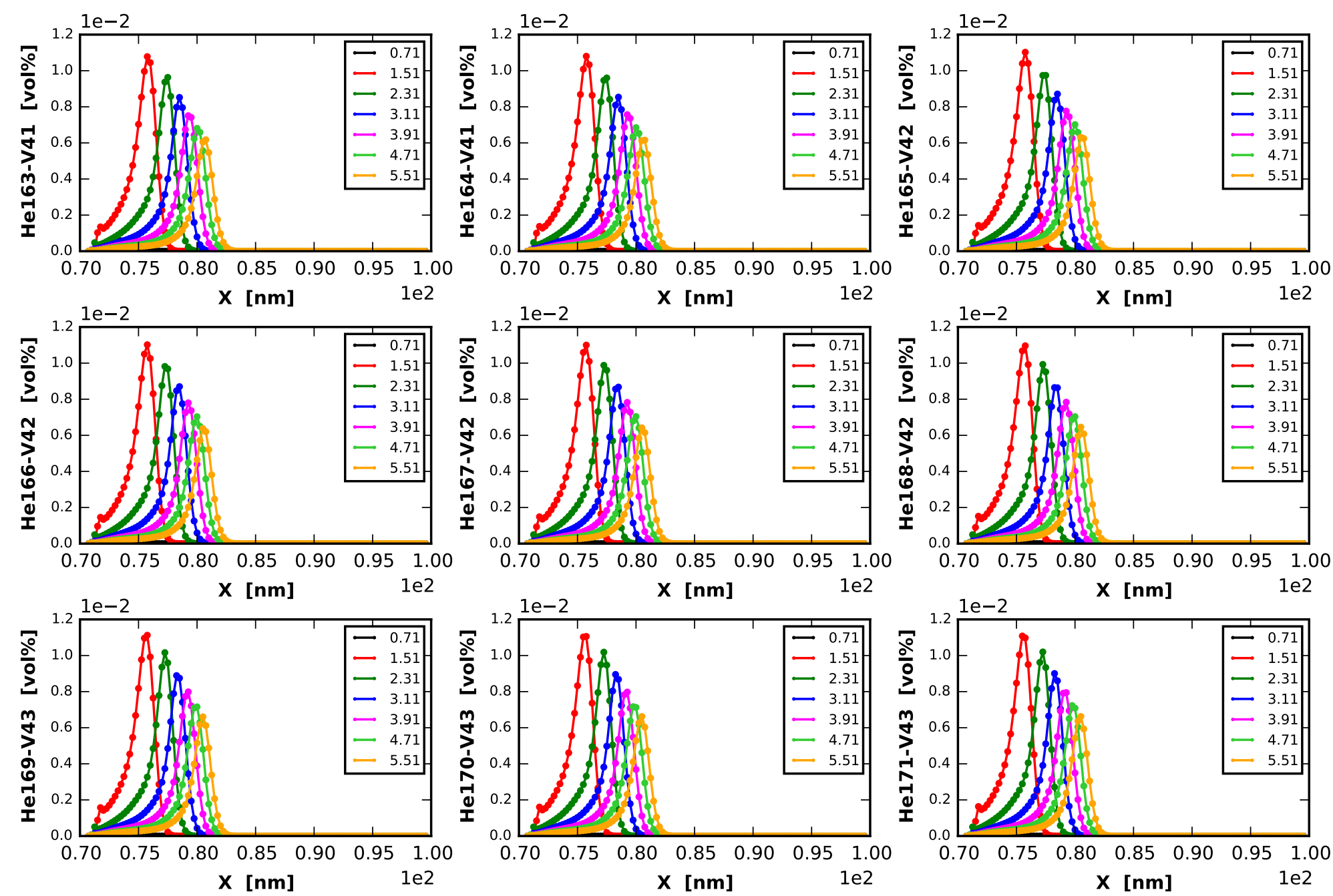
Transient: $[0.71,1.51,2.31,3.11,3.91,4.71,5.51]$ [us]
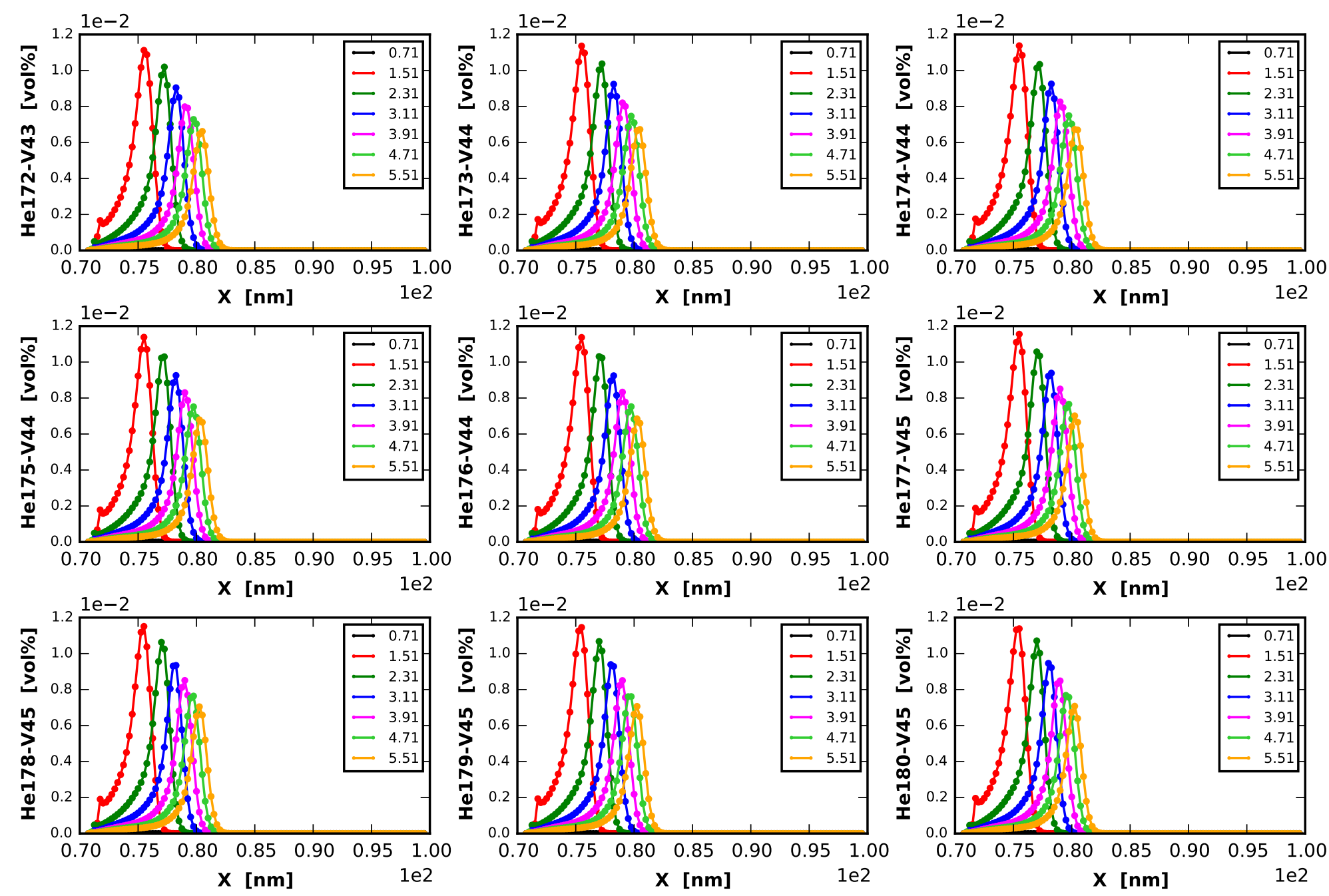
Transient: $[0.71,1.51,2.31,3.11,3.91,4.71,5.51]$ [us]
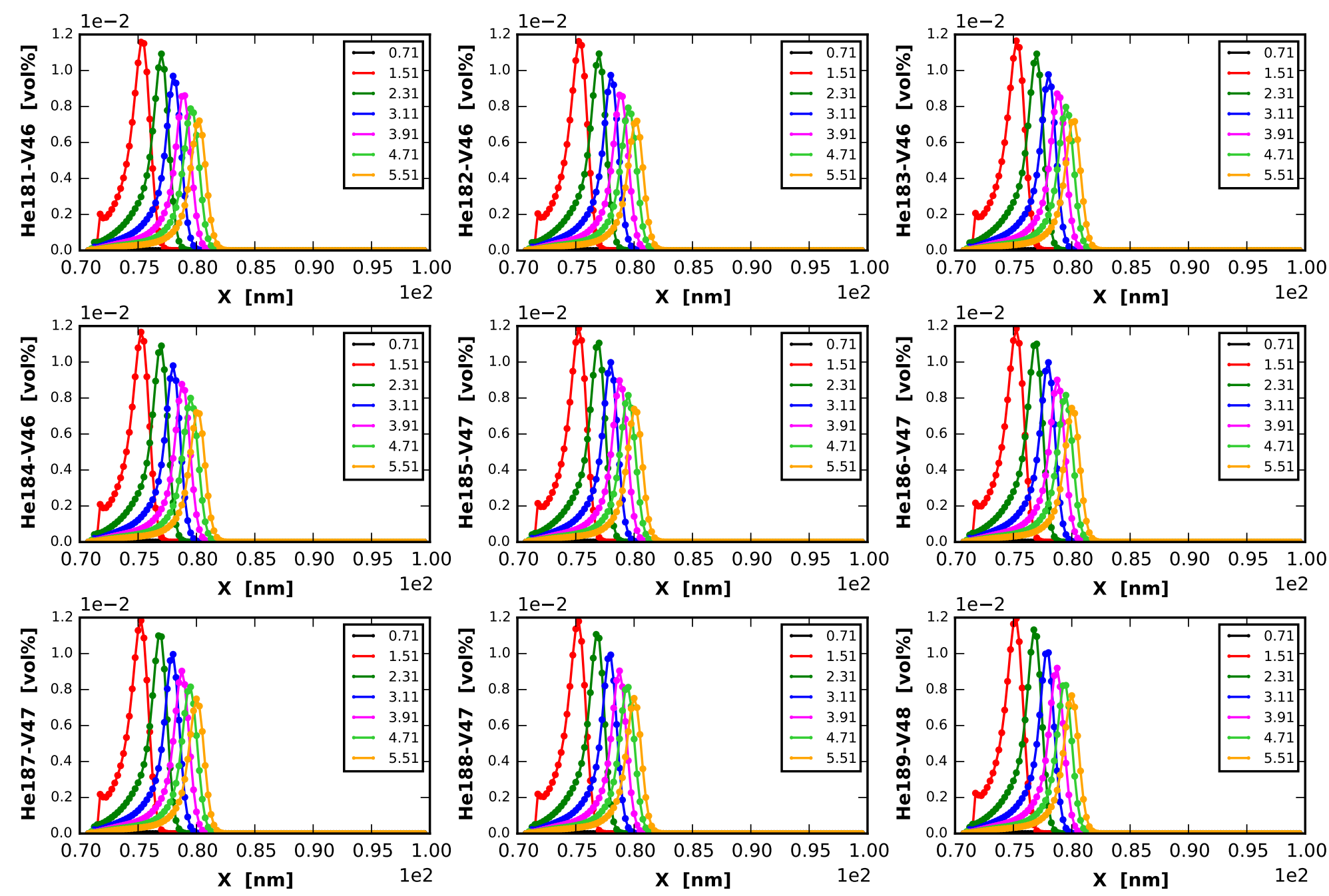
Transient: $[0.71,1.51,2.31,3.11,3.91,4.71,5.51]$ [us]
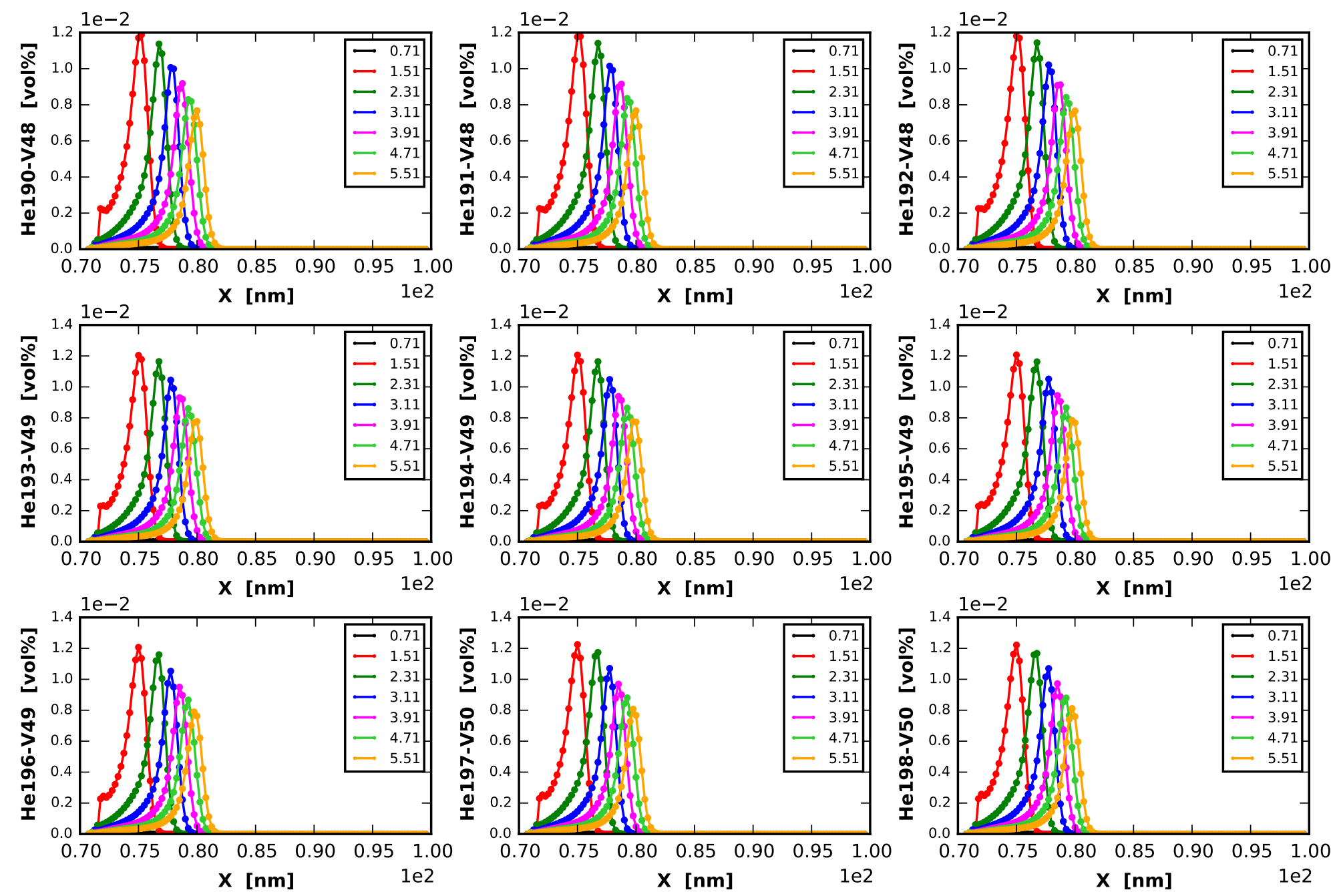
Transient: [0.71, 1.51, 2.31, 3.11, 3.91, 4.71, 5.51] [us]
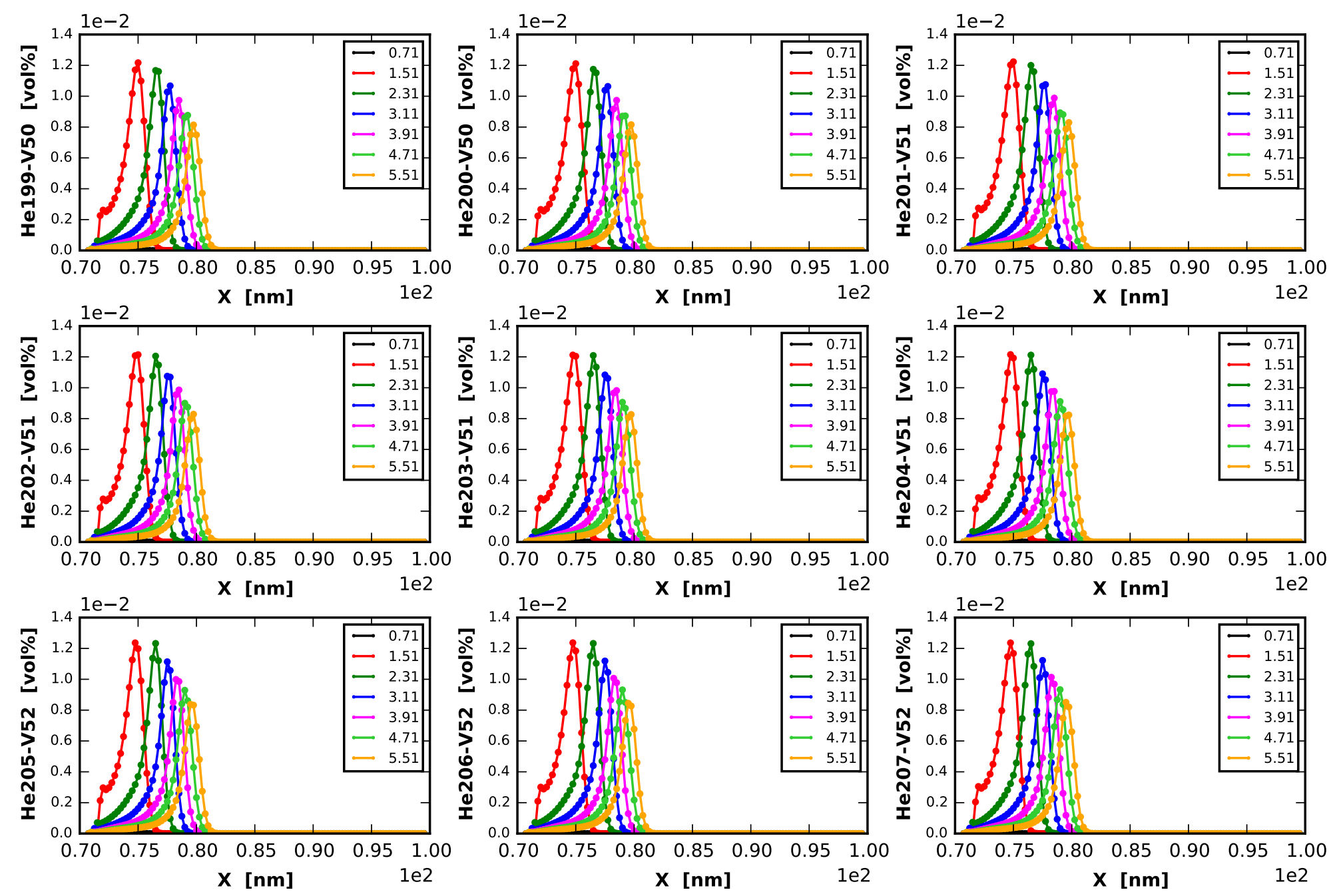
Transient: $[0.71,1.51,2.31,3.11,3.91,4.71,5.51]$ [us]
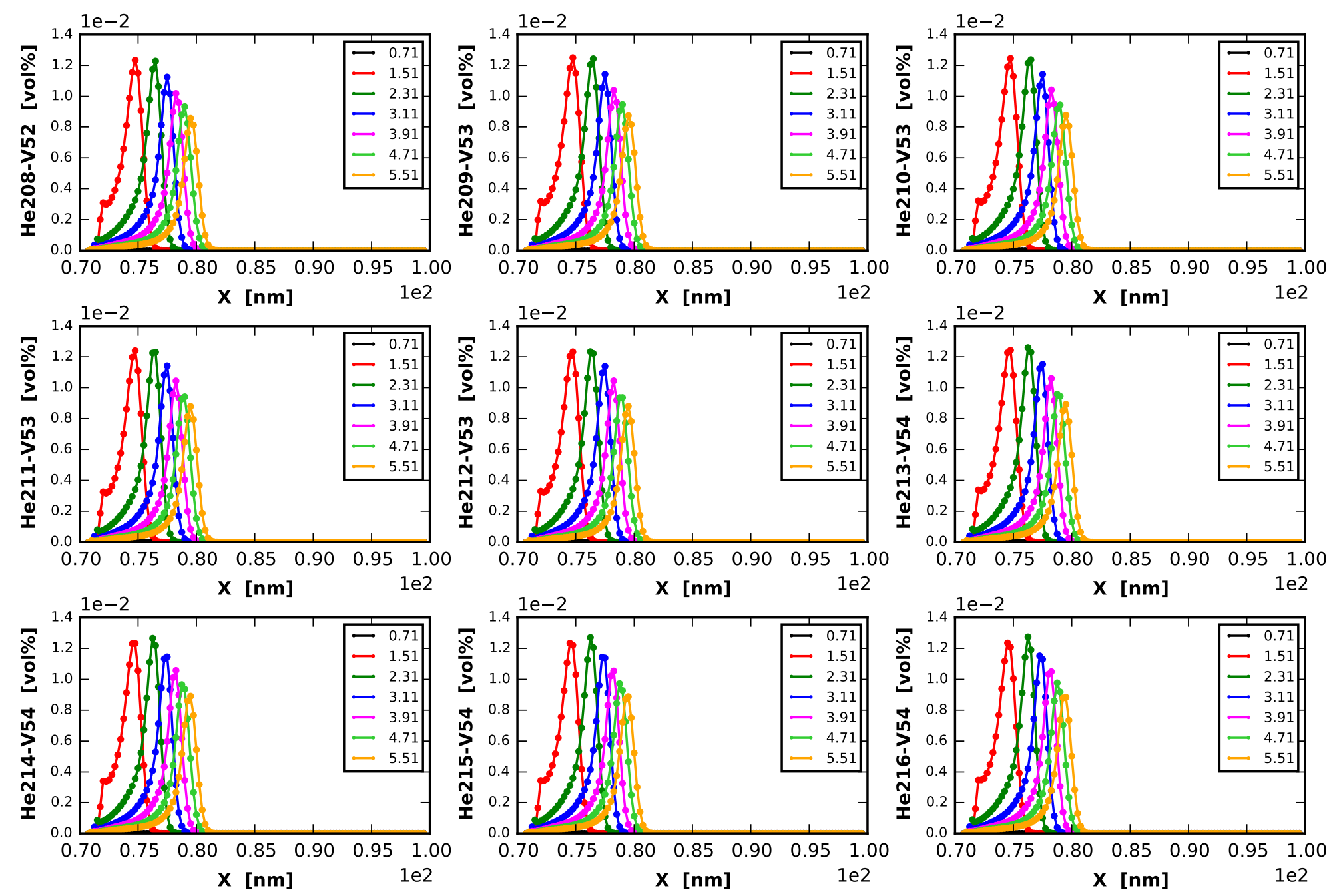
Transient: $[0.71,1.51,2.31,3.11,3.91,4.71,5.51]$ [us]
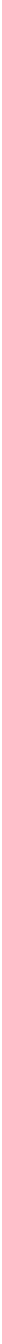
Transient: $[0.71,1.51,2.31,3.11,3.91,4.71,5.51]$ [us]
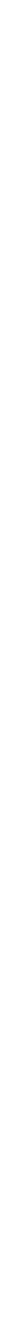
Transient: $[0.71,1.51,2.31,3.11,3.91,4.71,5.51]$ [us]
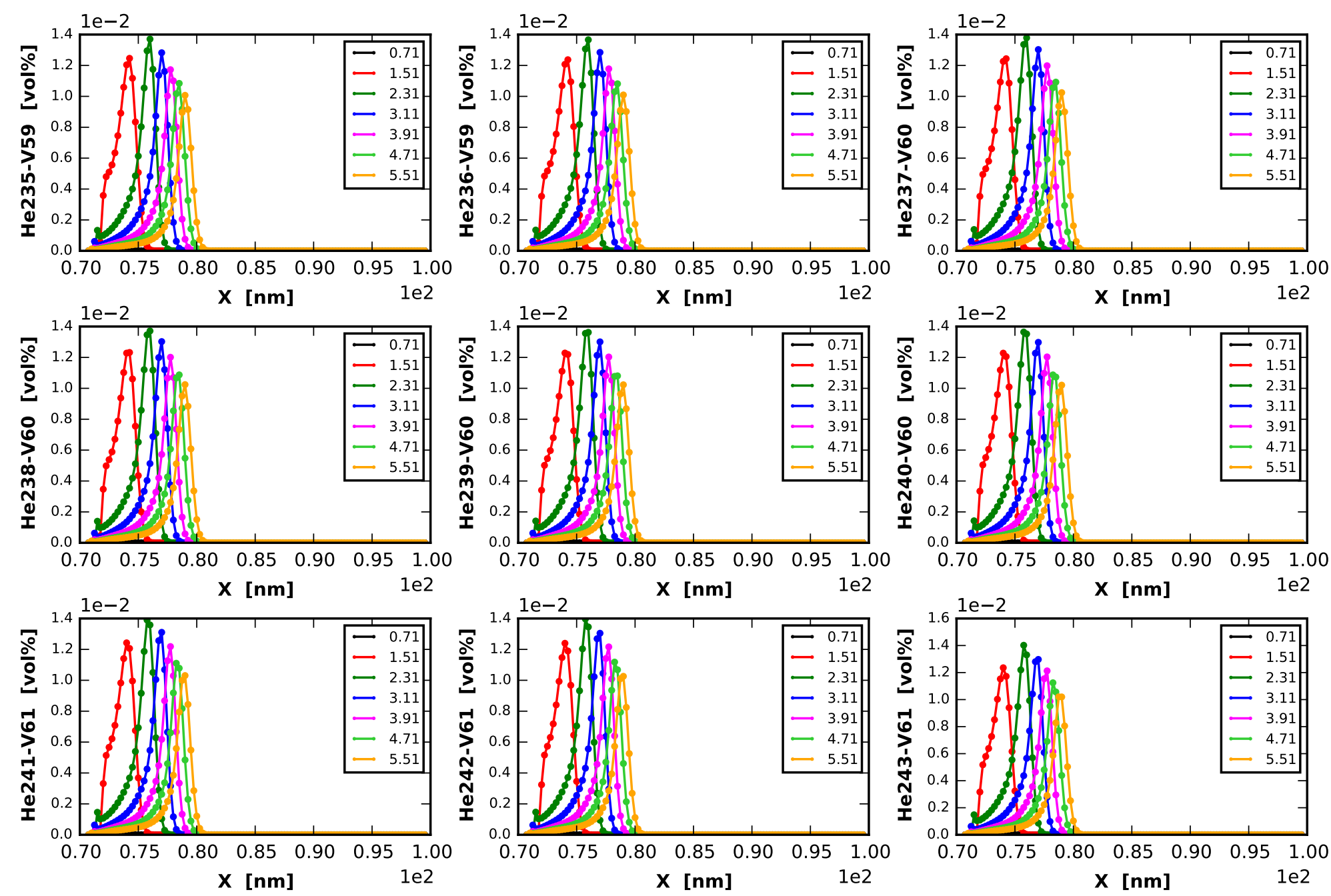
Transient: $[0.71,1.51,2.31,3.11,3.91,4.71,5.51]$ [us]
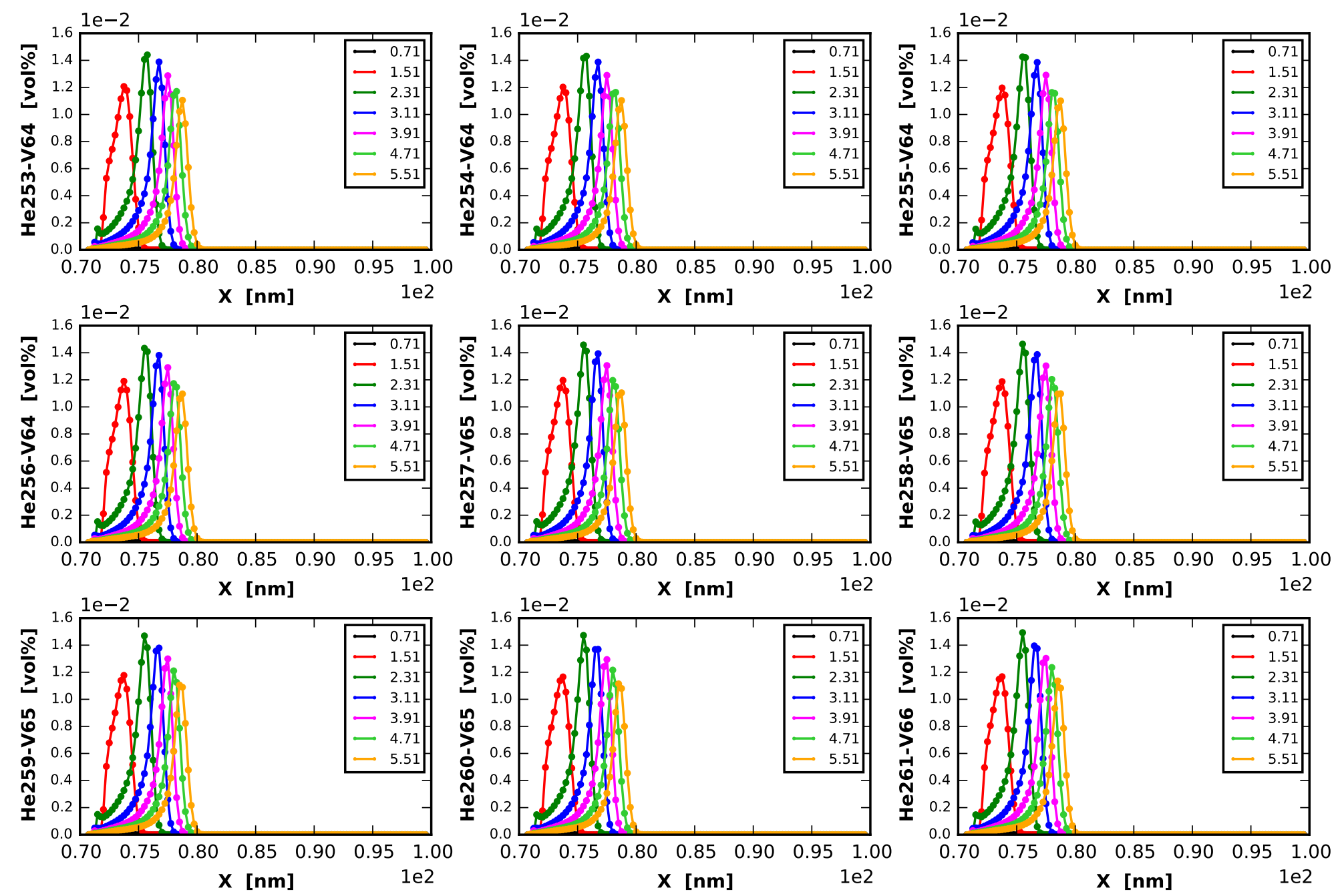
Transient: $[0.71,1.51,2.31,3.11,3.91,4.71,5.51]$ [us]
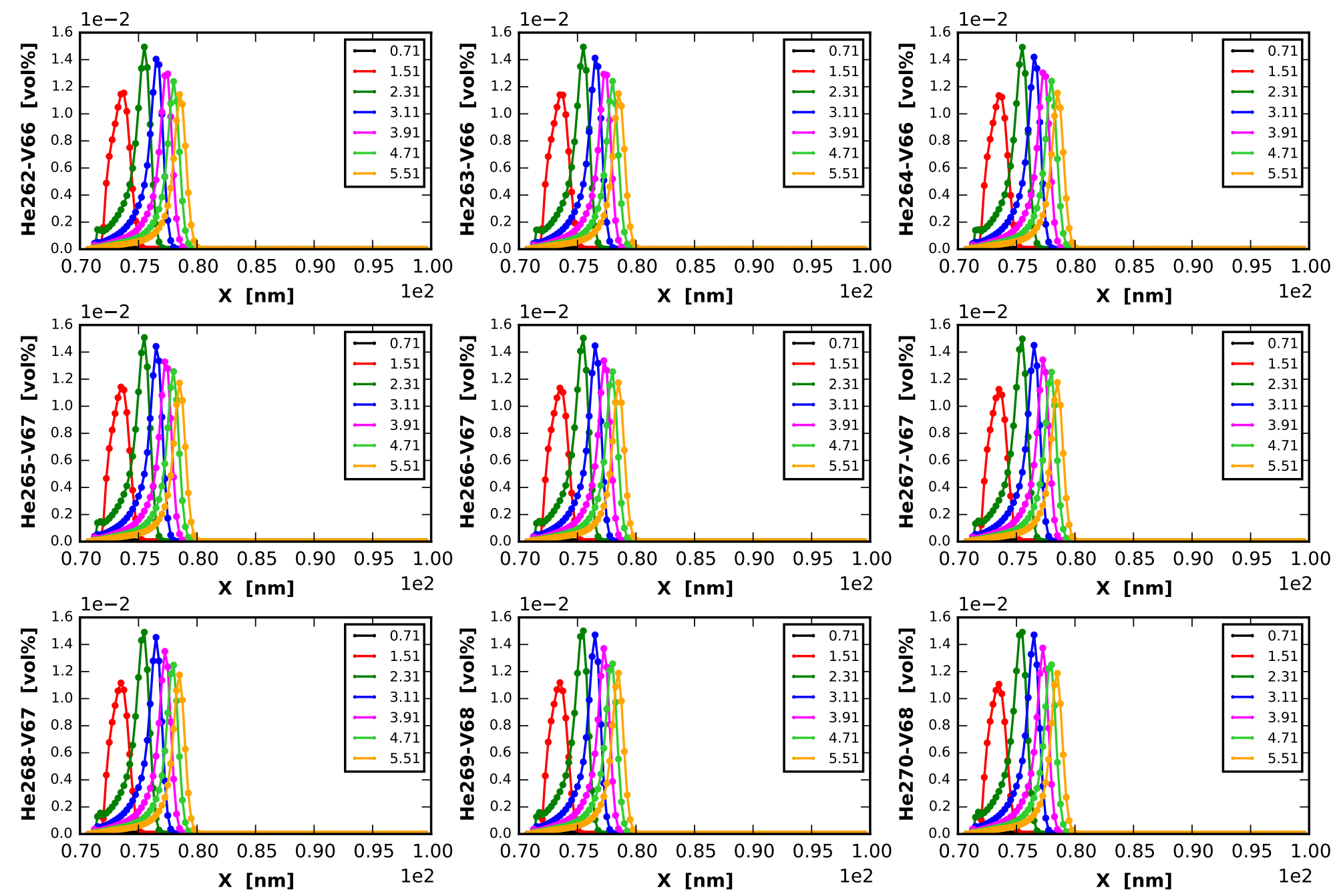
Transient: $[0.71,1.51,2.31,3.11,3.91,4.71,5.51]$ [us]
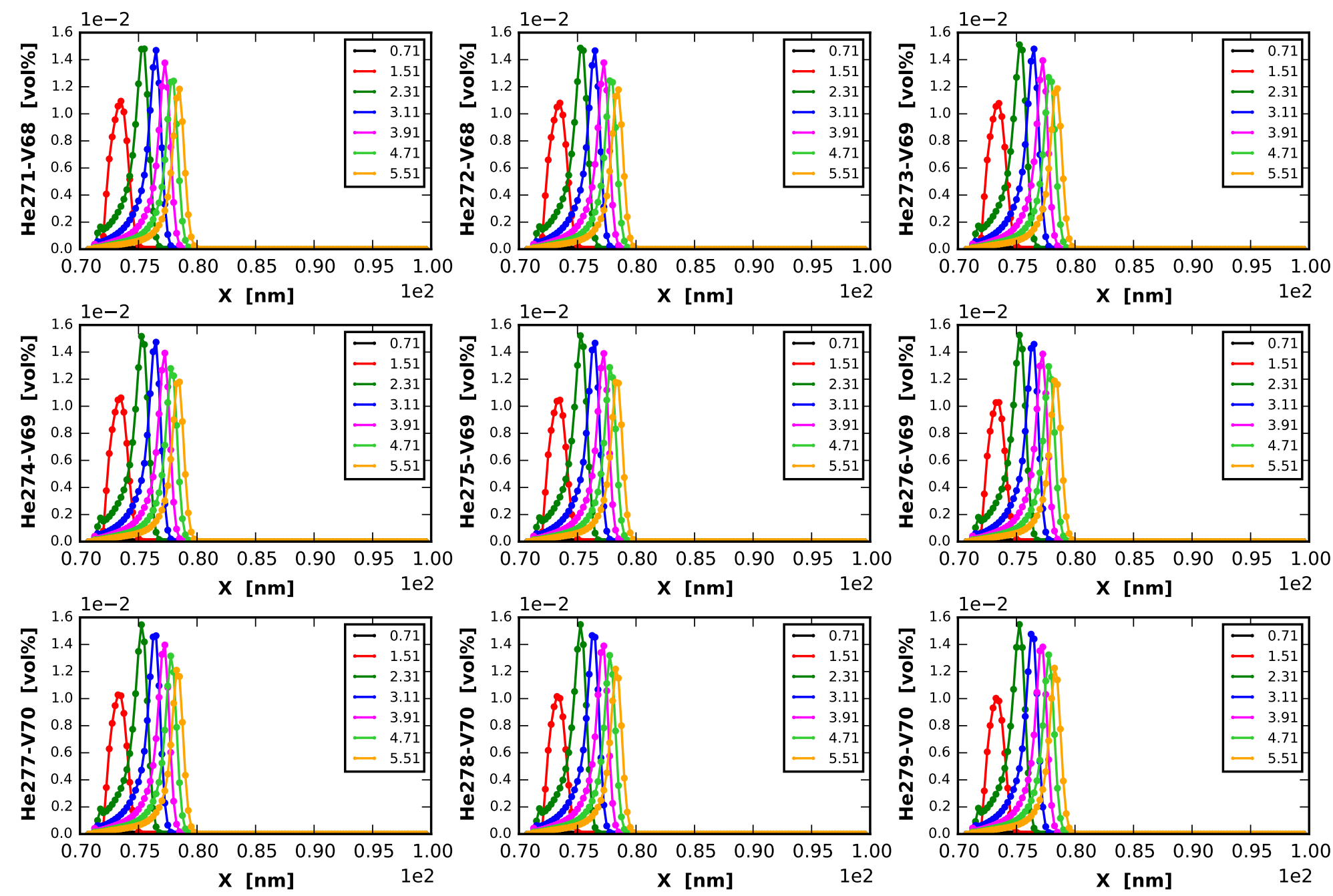
Transient: $[0.71,1.51,2.31,3.11,3.91,4.71,5.51]$ [us]
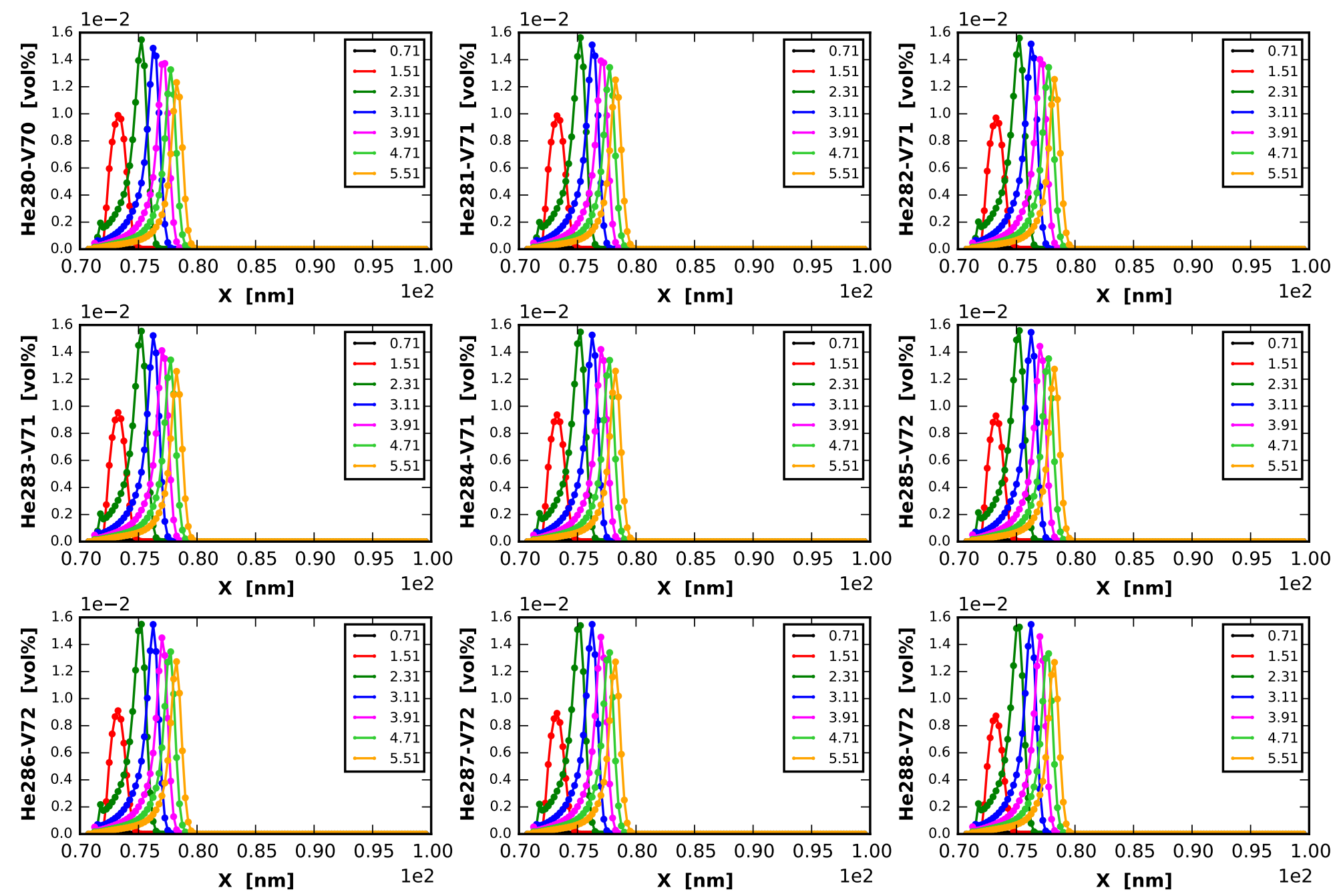
Transient: $[0.71,1.51,2.31,3.11,3.91,4.71,5.51]$ [us]
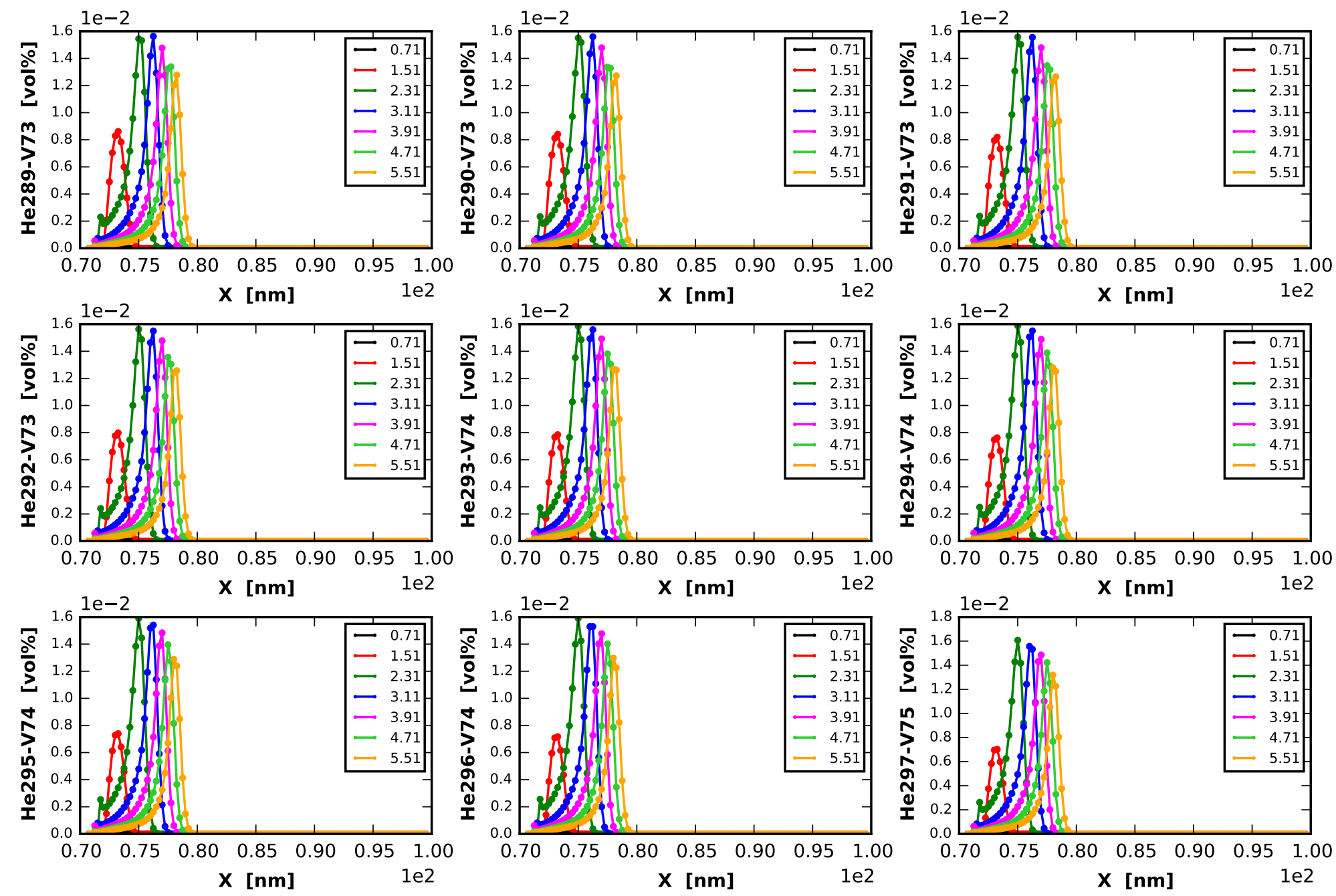
Transient: $[0.71,1.51,2.31,3.11,3.91,4.71,5.51]$ [us]
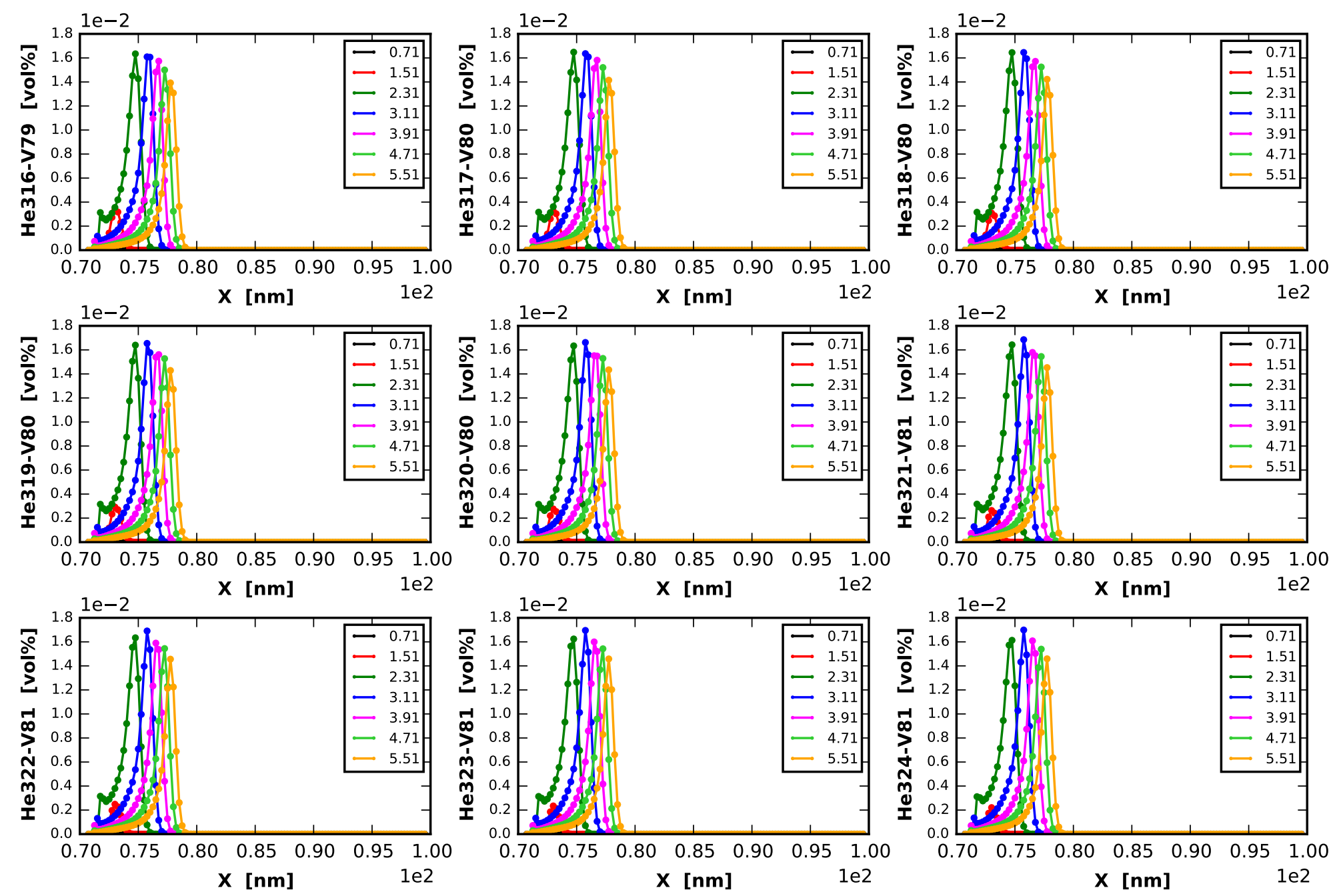
Transient: [0.71, 1.51, 2.31, 3.11, 3.91, 4.71, 5.51] [us]
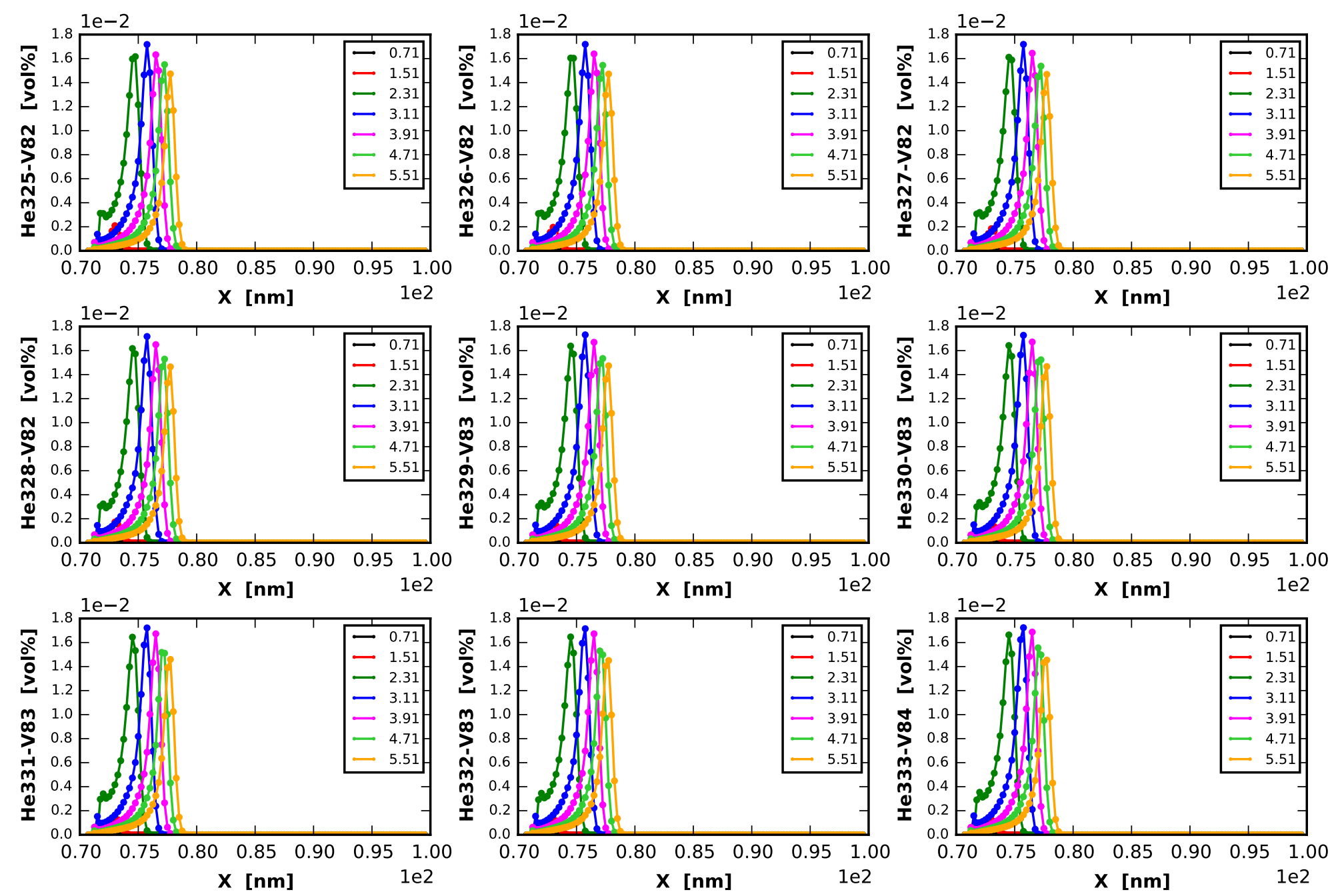
Transient: $[0.71,1.51,2.31,3.11,3.91,4.71,5.51]$ [us]
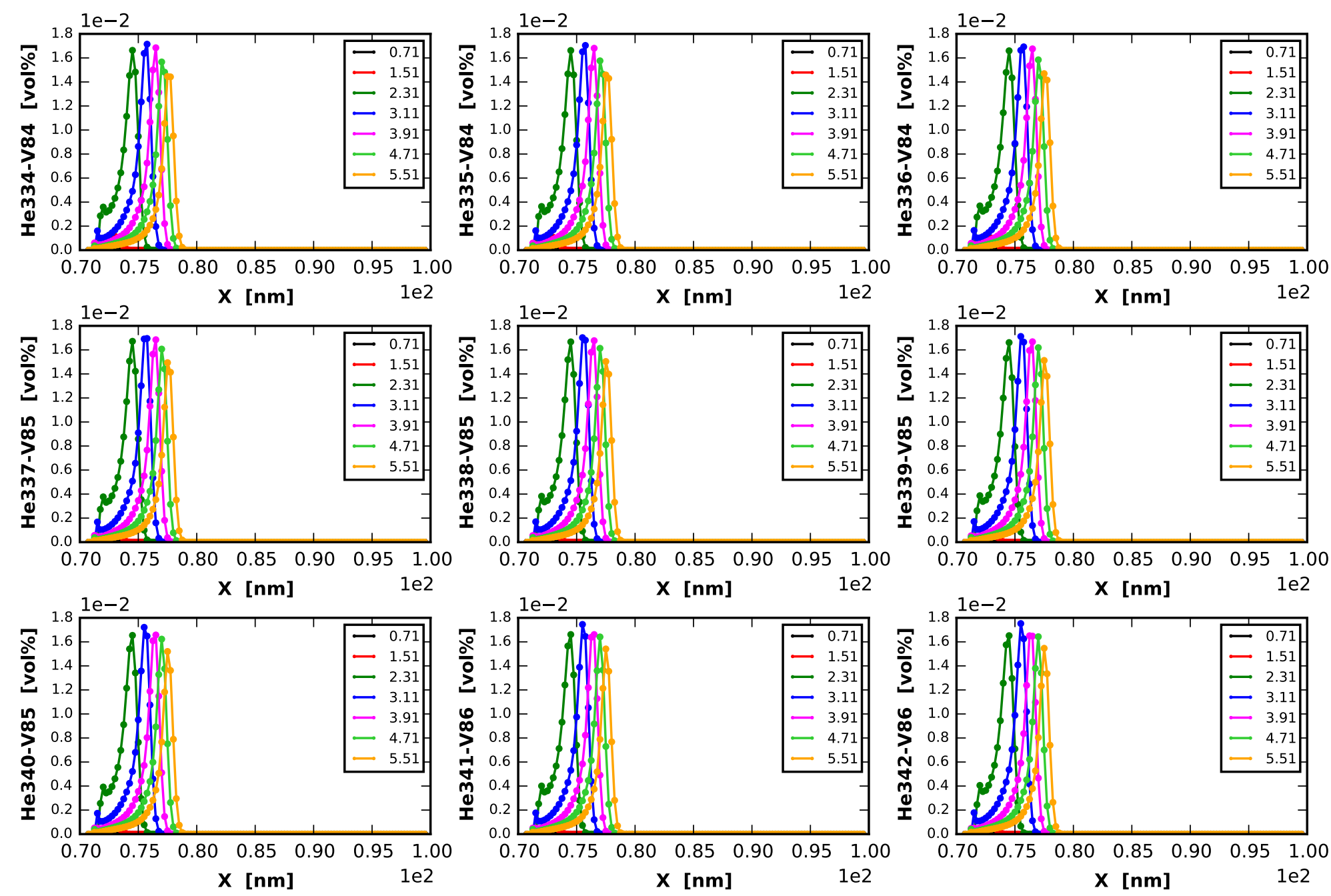
Transient: $[0.71,1.51,2.31,3.11,3.91,4.71,5.51]$ [us]
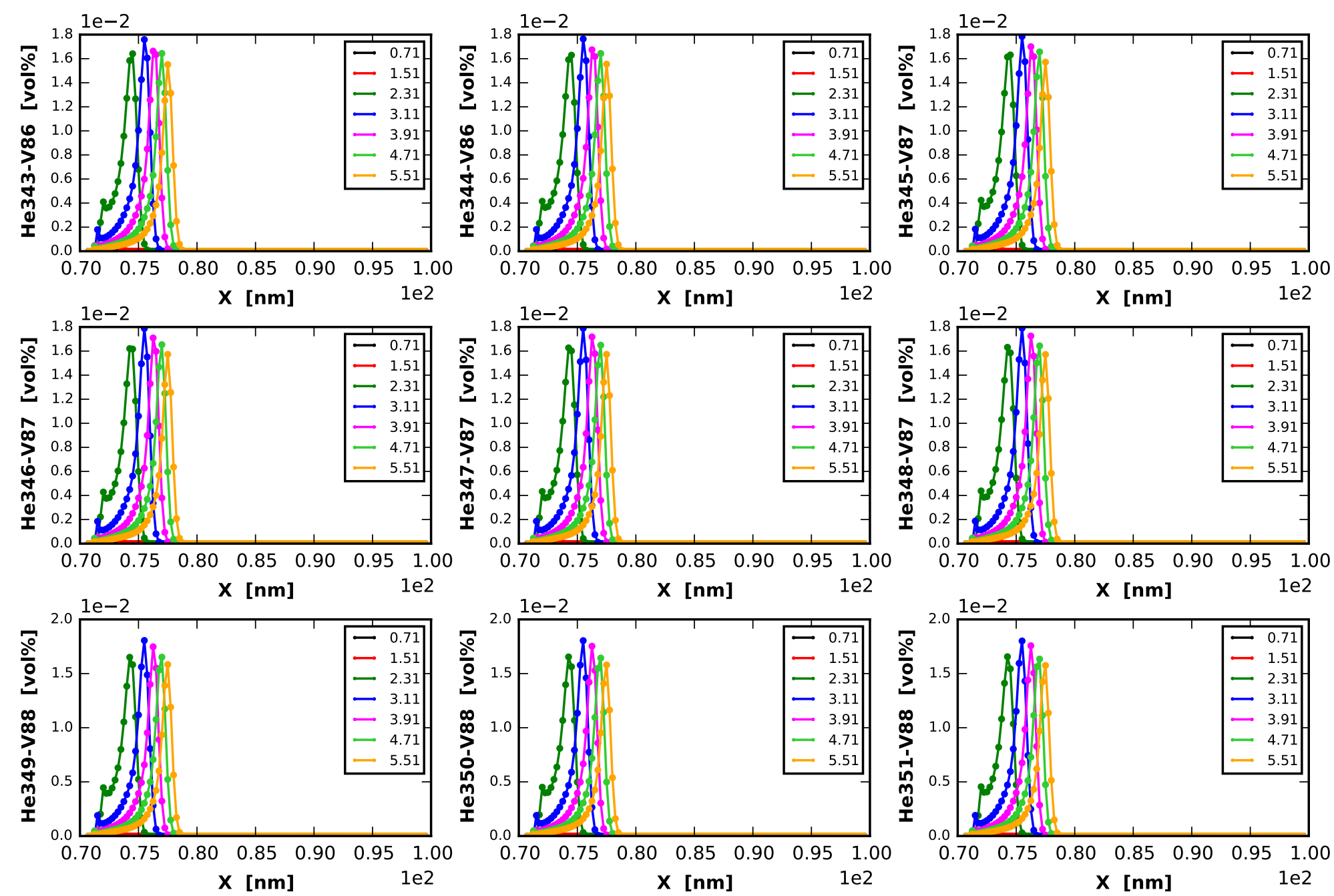
Transient: [0.71, 1.51, 2.31, 3.11, 3.91, 4.71, 5.51] [us]
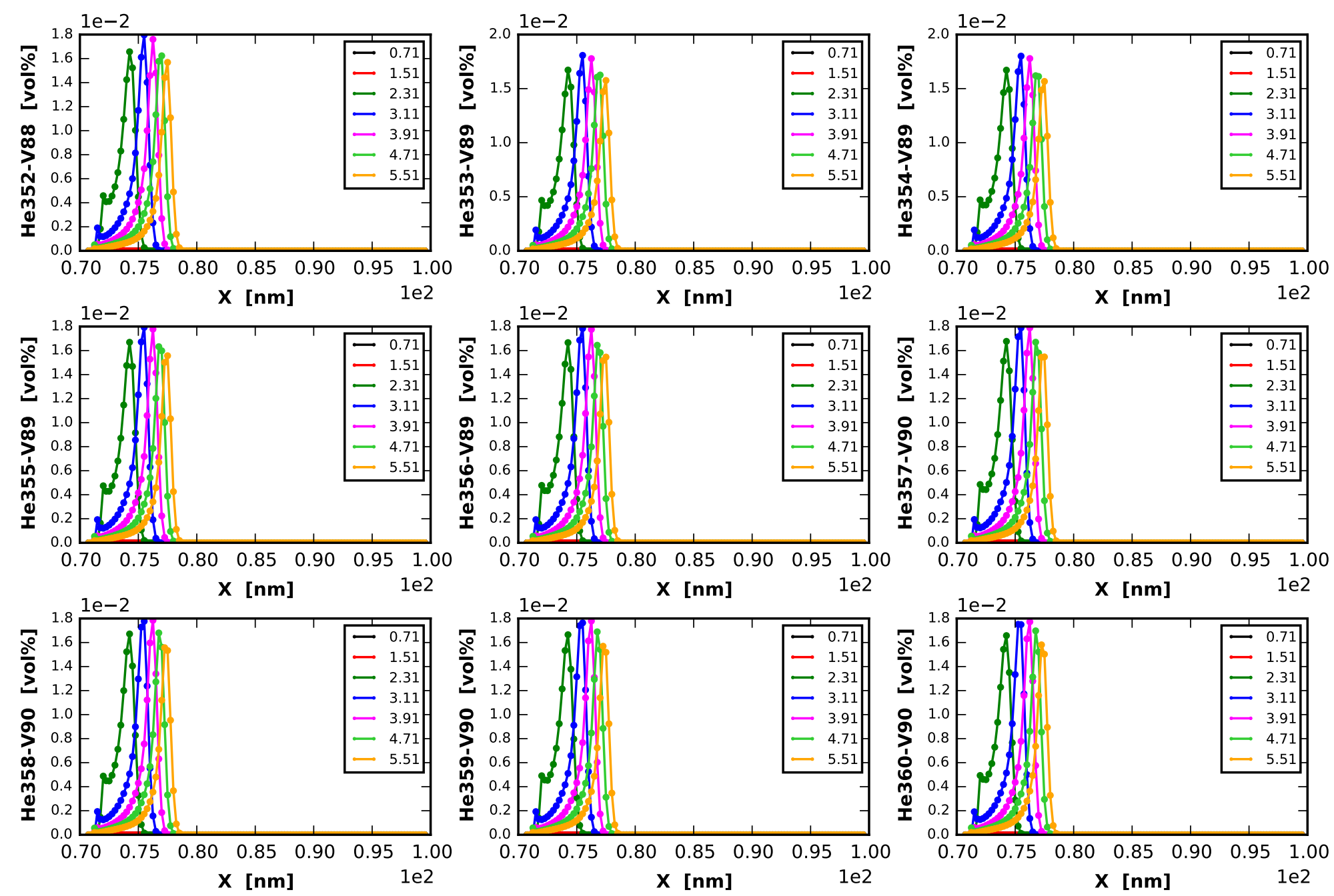
Transient: $[0.71,1.51,2.31,3.11,3.91,4.71,5.51]$ [us]
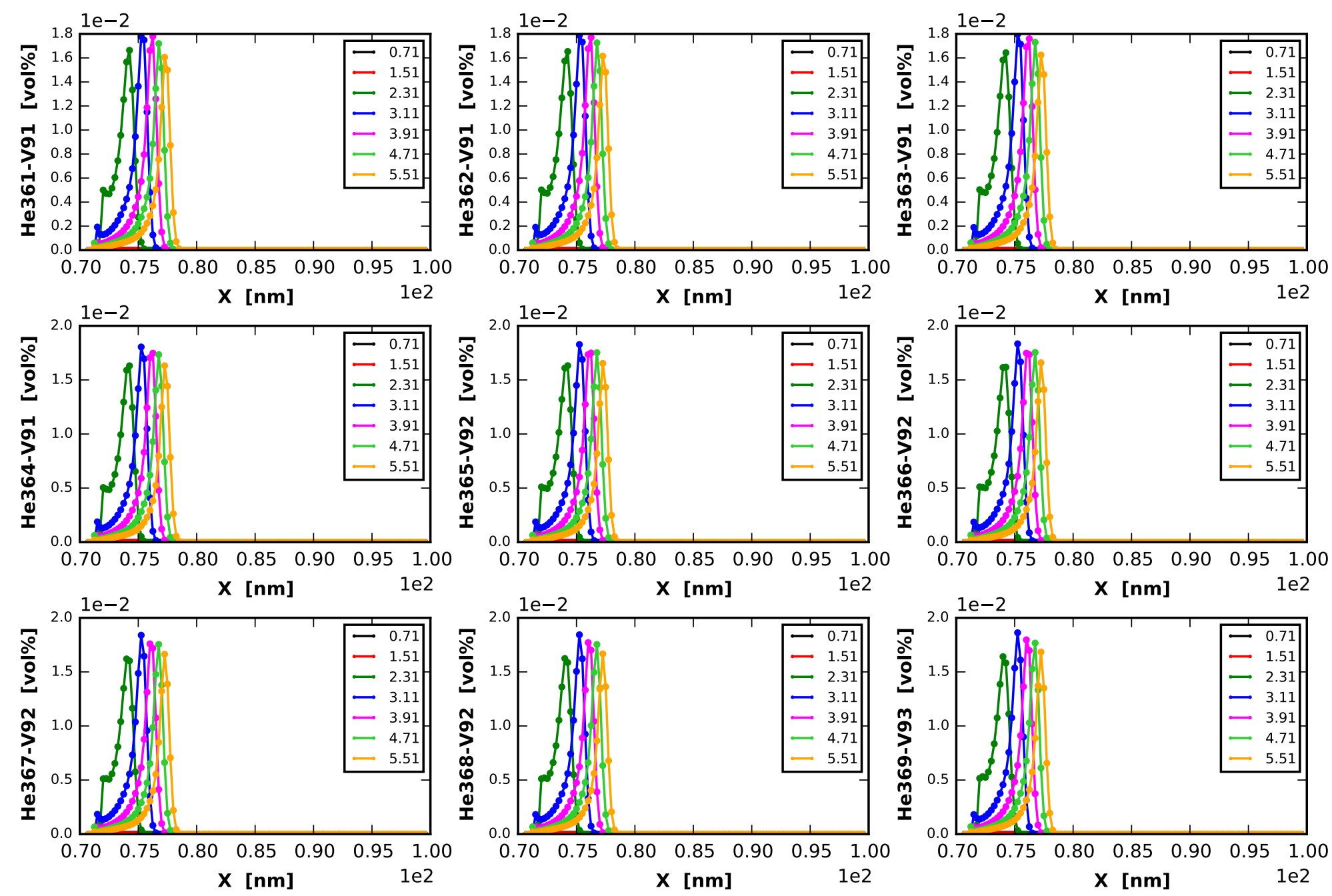
Transient: $[0.71,1.51,2.31,3.11,3.91,4.71,5.51]$ [us]
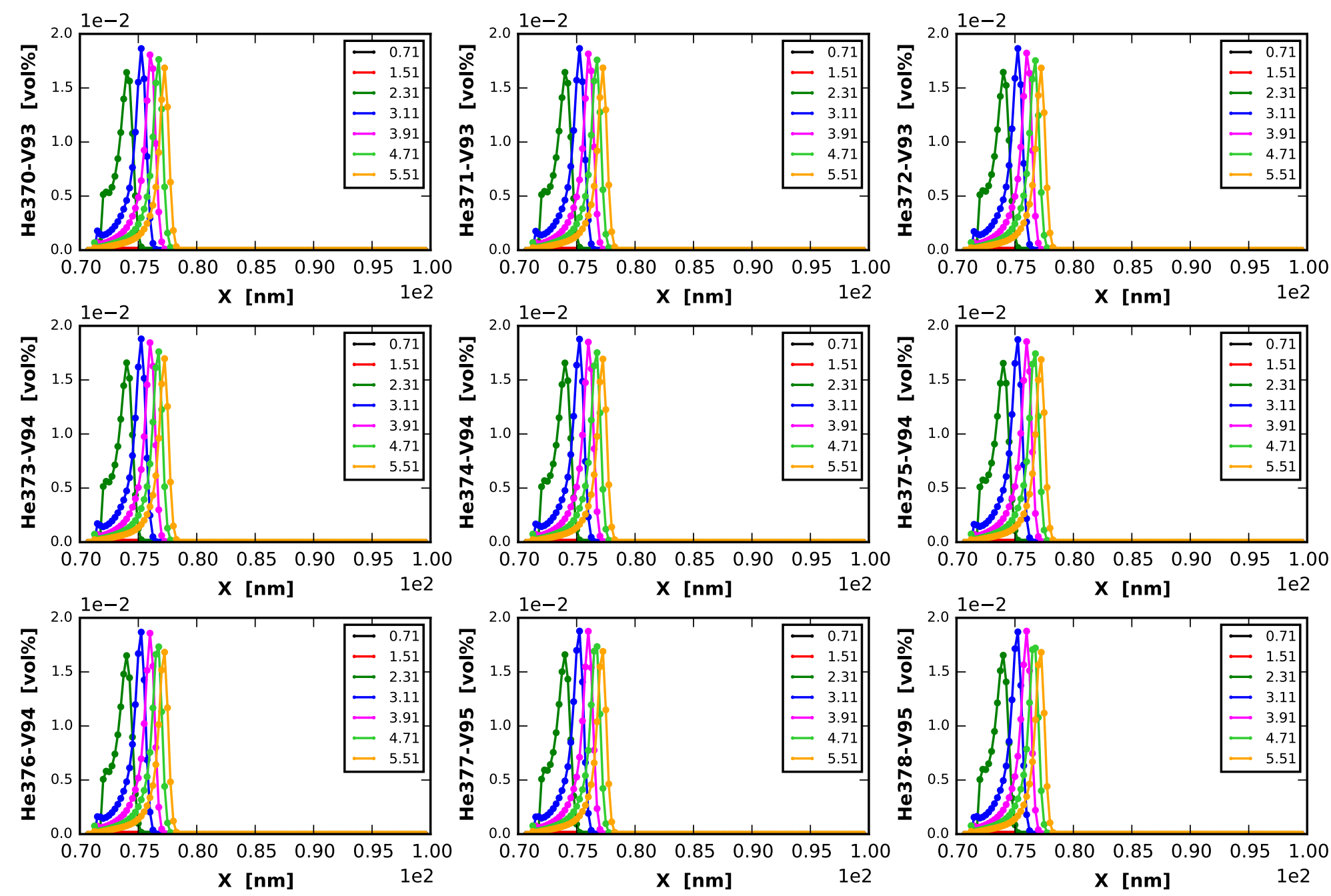
Transient: $[0.71,1.51,2.31,3.11,3.91,4.71,5.51]$ [us]
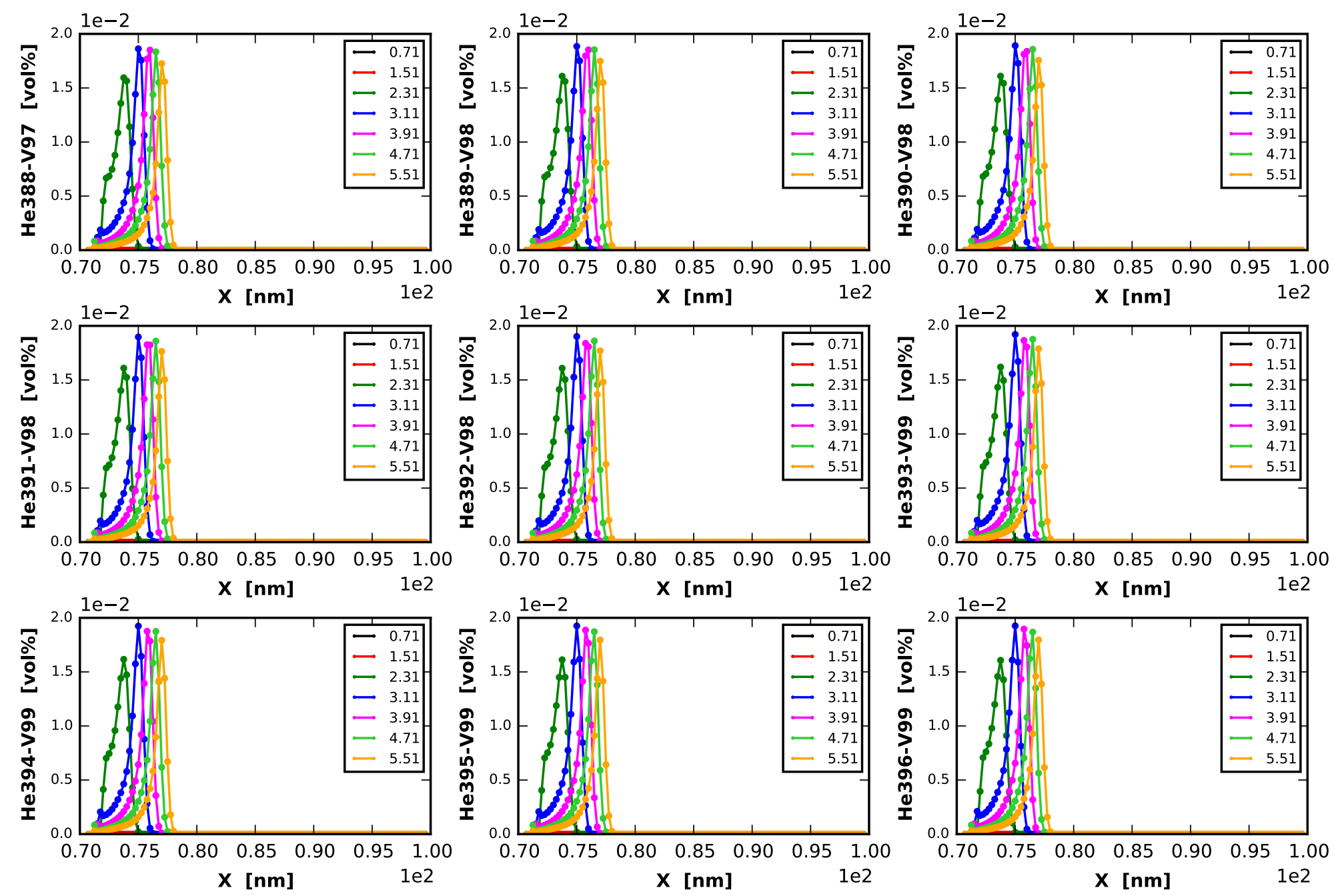
Transient: [0.71, 1.51, 2.31, 3.11, 3.91, 4.71, 5.51] [us]
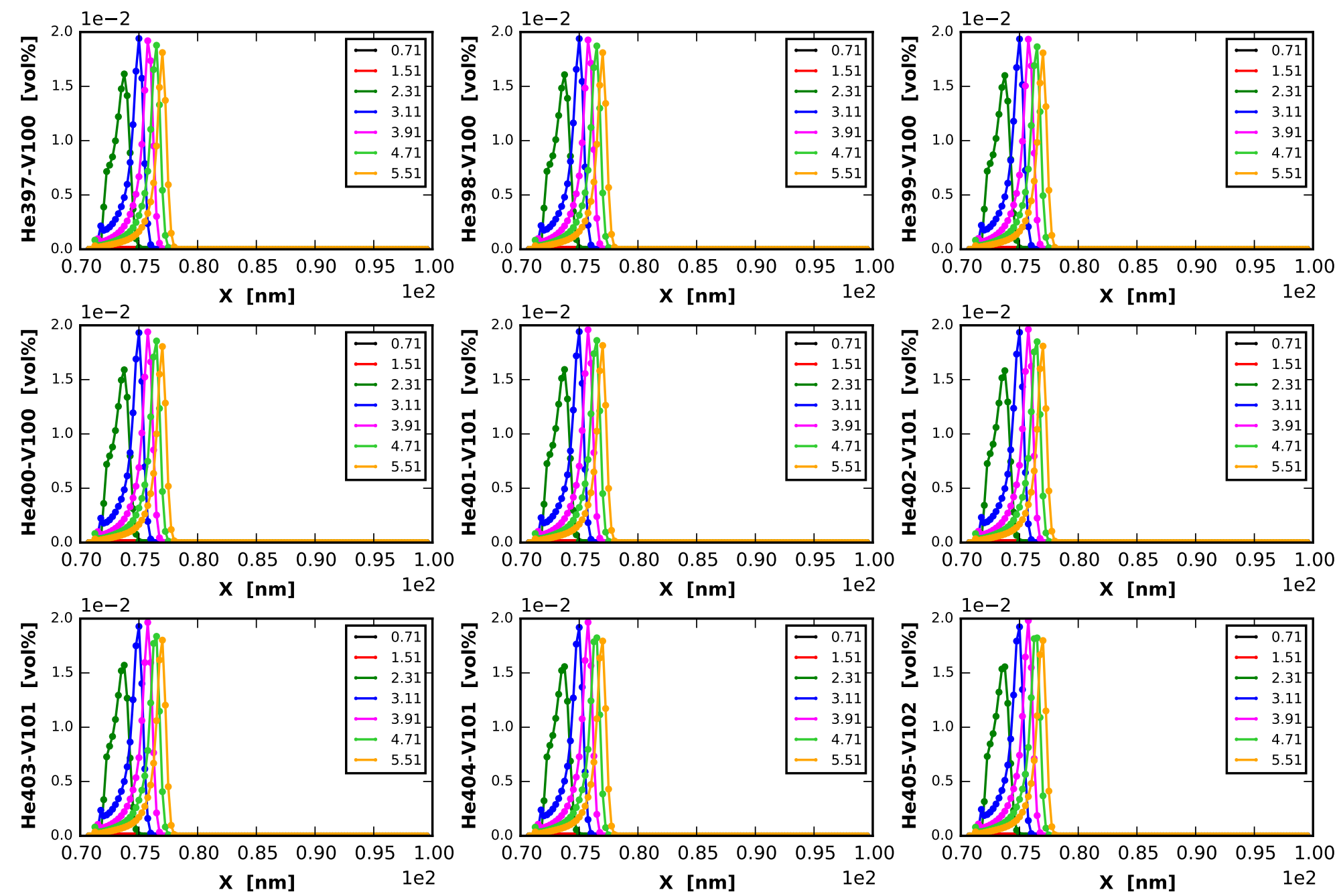
Transient: [0.71, 1.51, 2.31, 3.11, 3.91, 4.71, 5.51] [us]
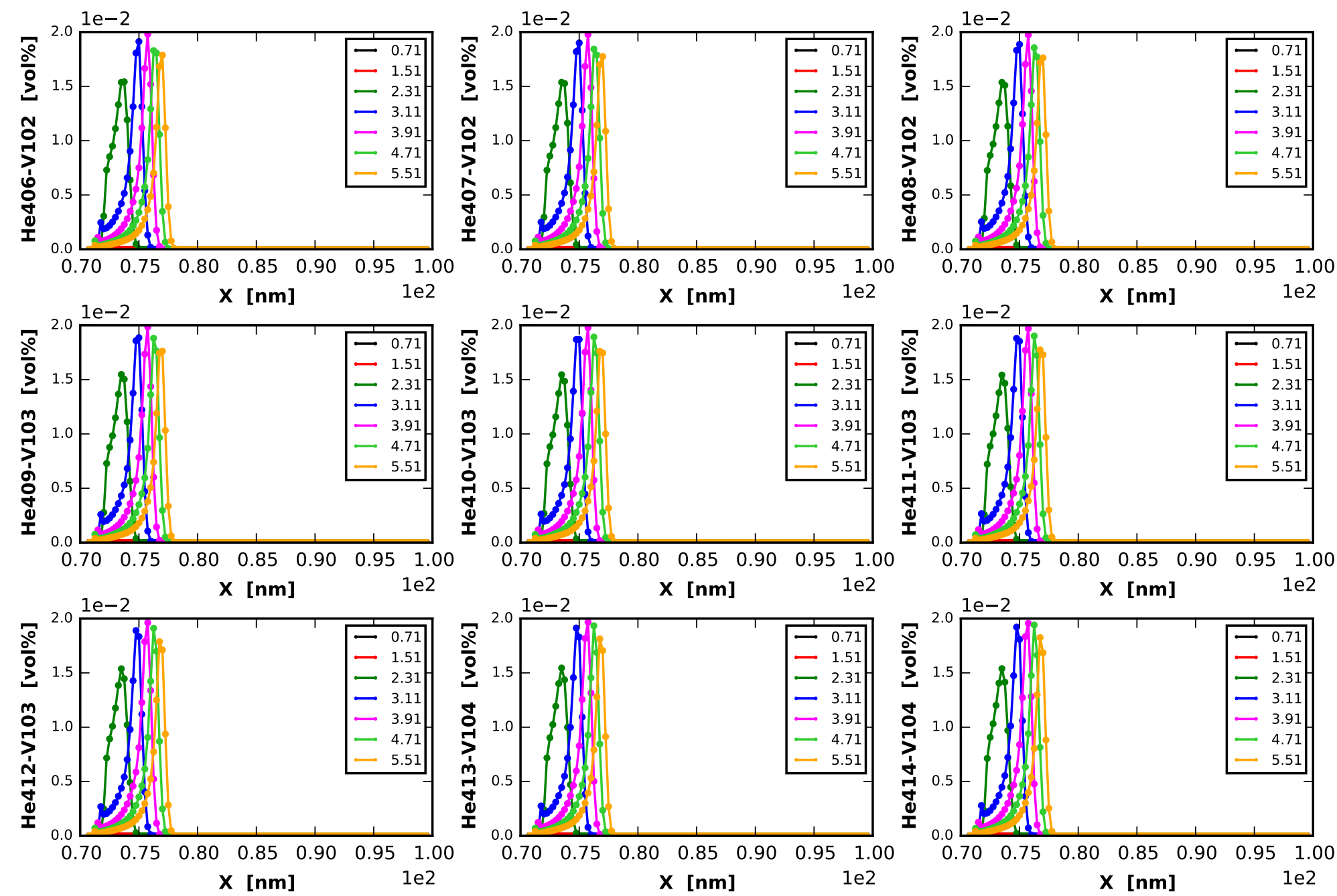
Transient: $[0.71,1.51,2.31,3.11,3.91,4.71,5.51]$ [us]
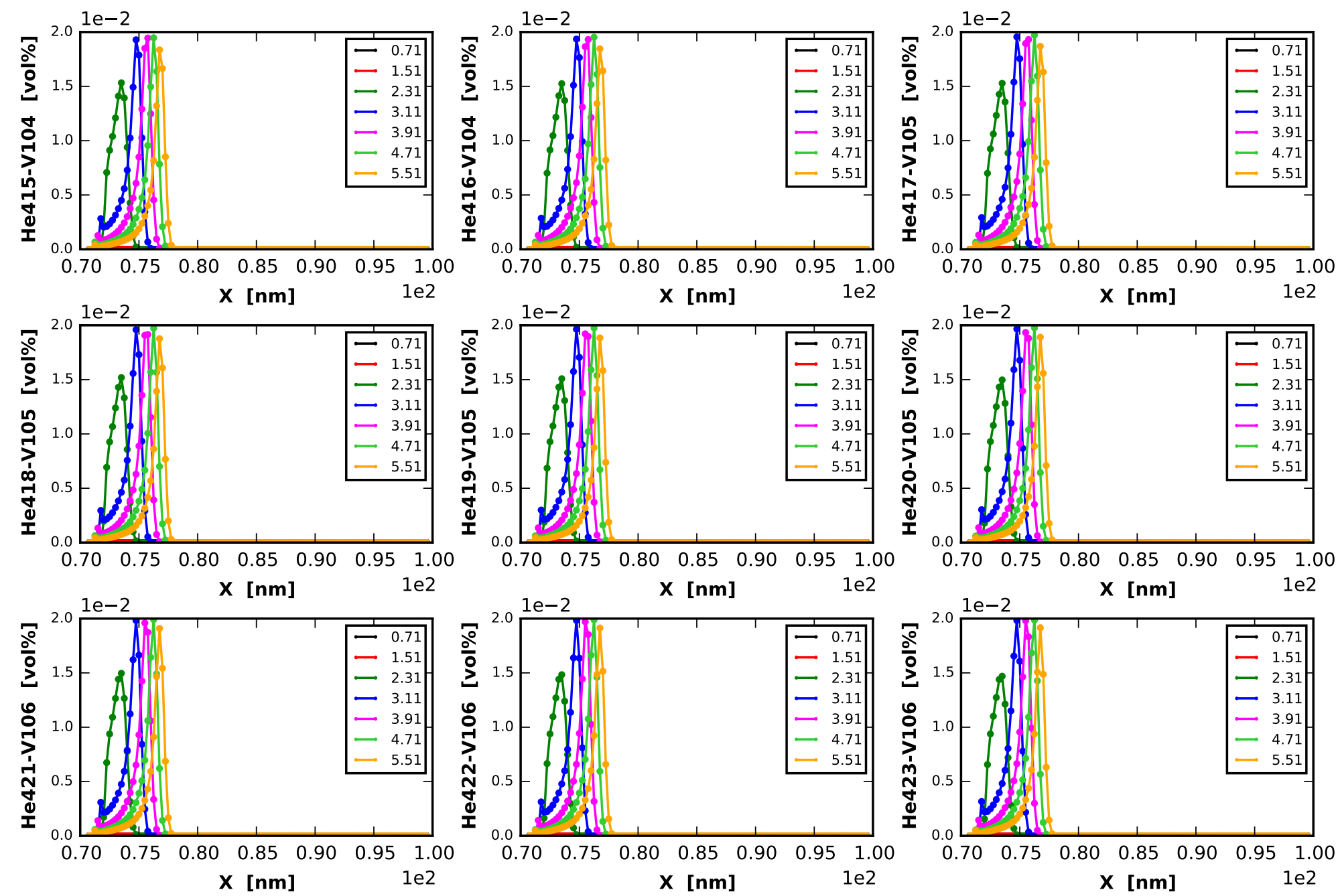
Transient: $[0.71,1.51,2.31,3.11,3.91,4.71,5.51]$ [us]
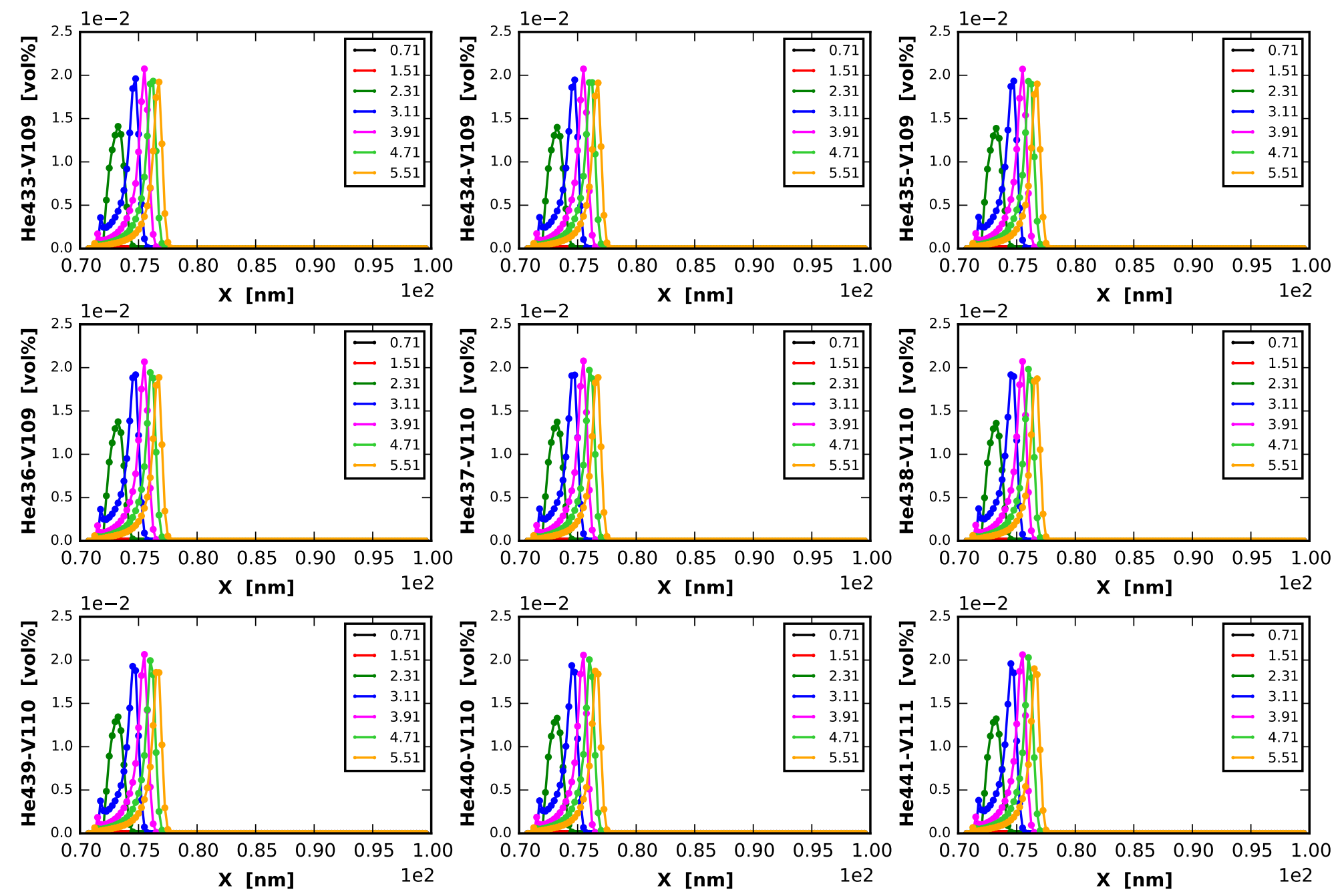
Transient: $[0.71,1.51,2.31,3.11,3.91,4.71,5.51]$ [us]
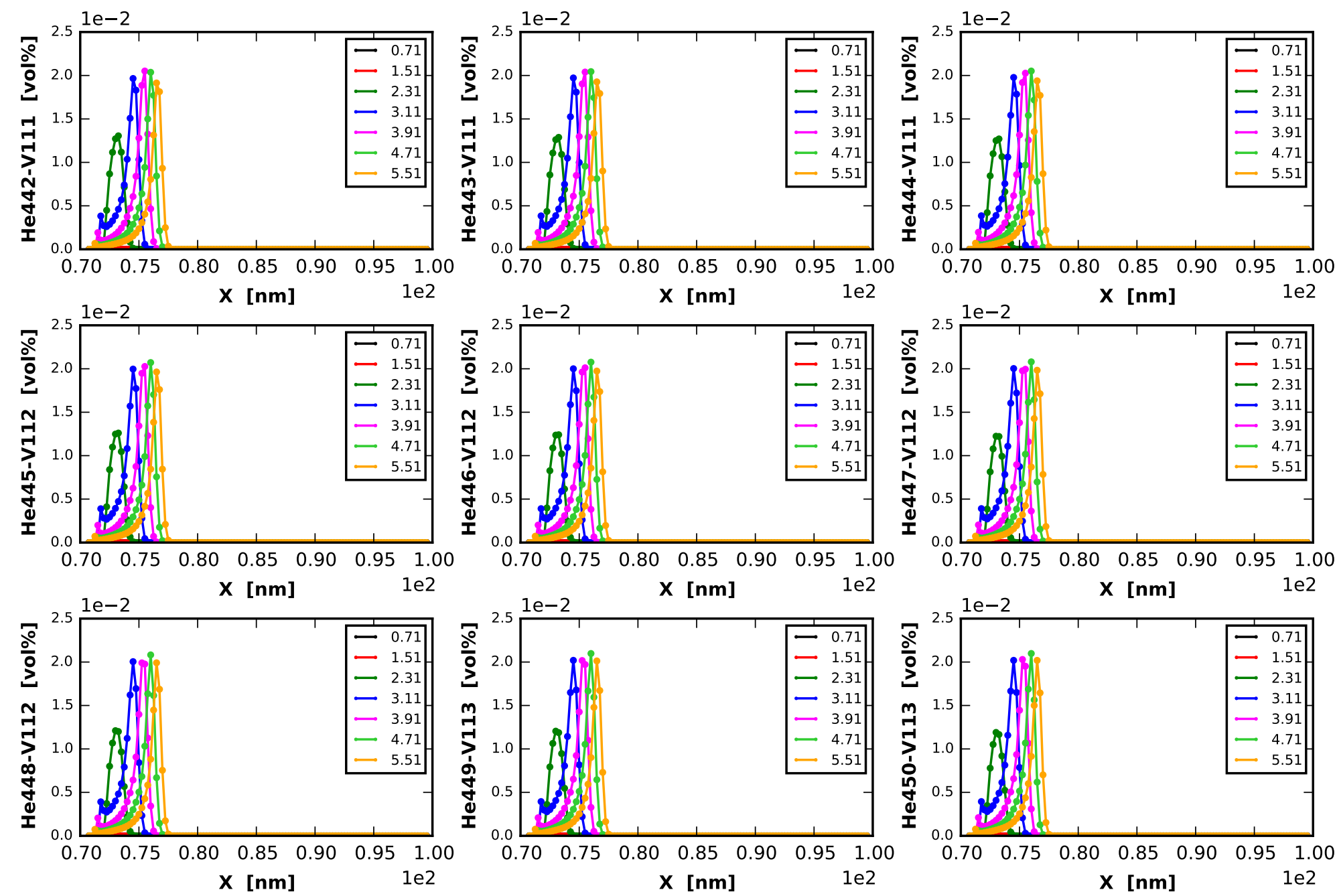
Transient: $[0.71,1.51,2.31,3.11,3.91,4.71,5.51]$ [us]
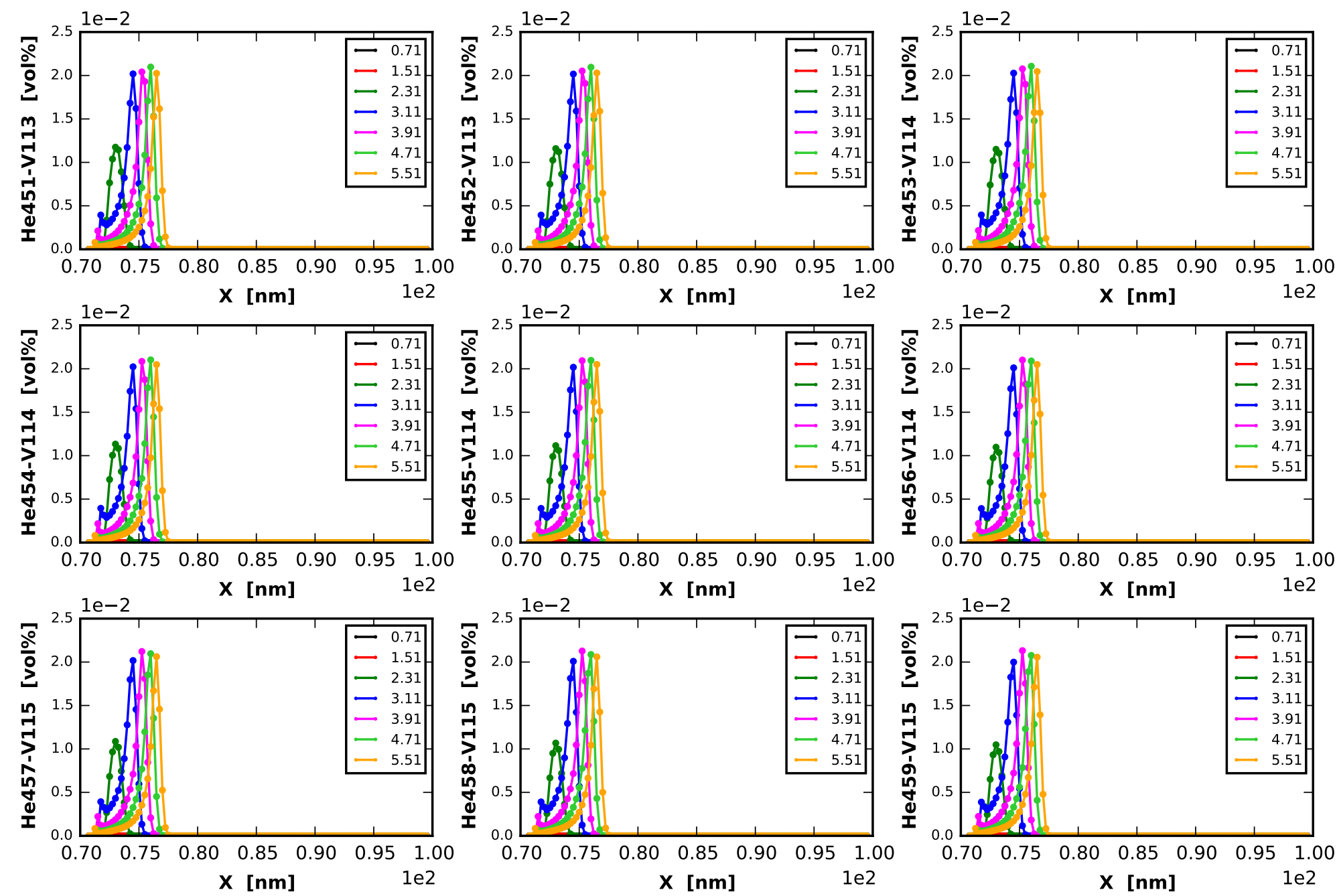
Transient: $[0.71,1.51,2.31,3.11,3.91,4.71,5.51]$ [us]
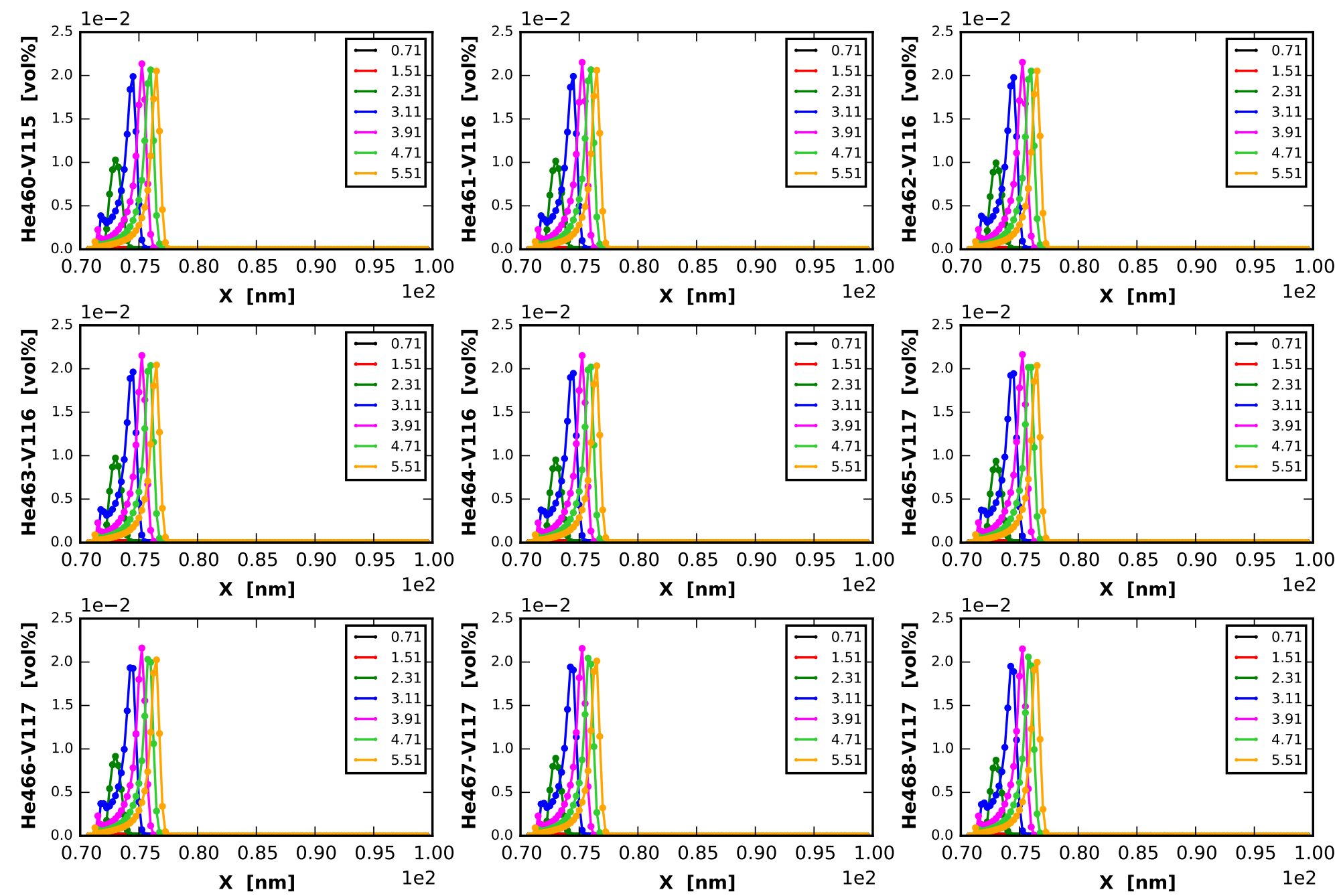
Transient: $[0.71,1.51,2.31,3.11,3.91,4.71,5.51]$ [us]
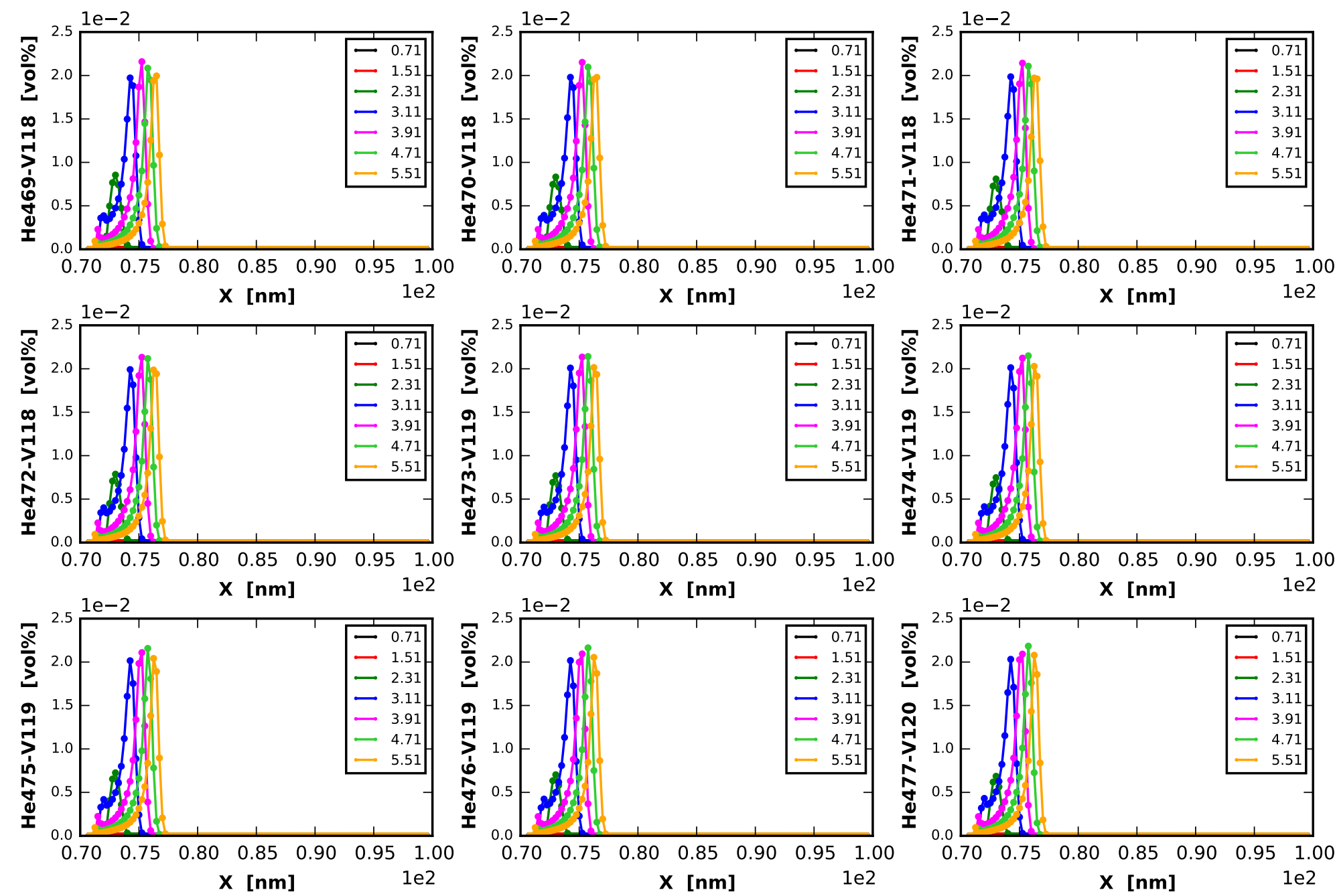
Transient: $[0.71,1.51,2.31,3.11,3.91,4.71,5.51]$ [us]
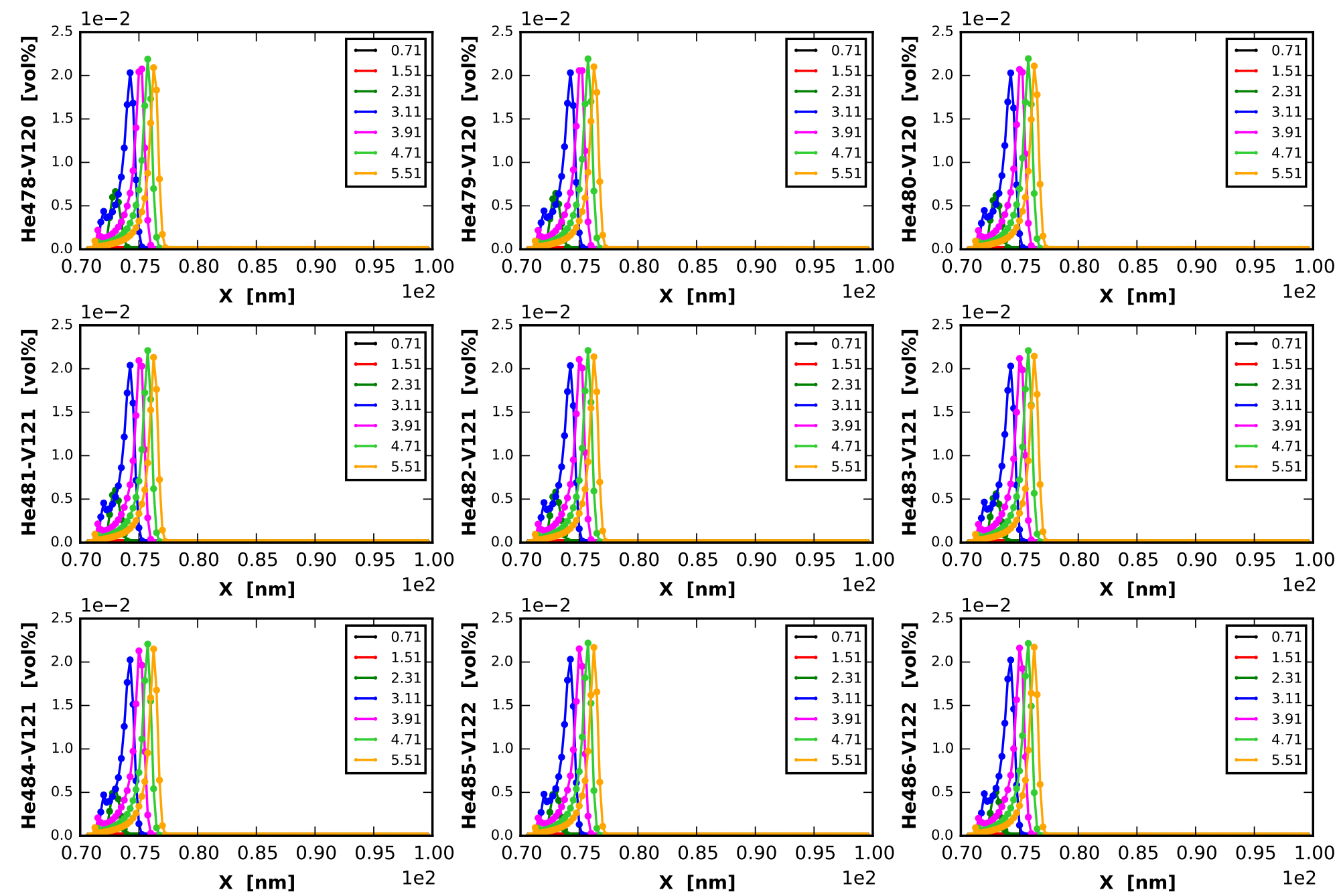
Transient: $[0.71,1.51,2.31,3.11,3.91,4.71,5.51]$ [us]
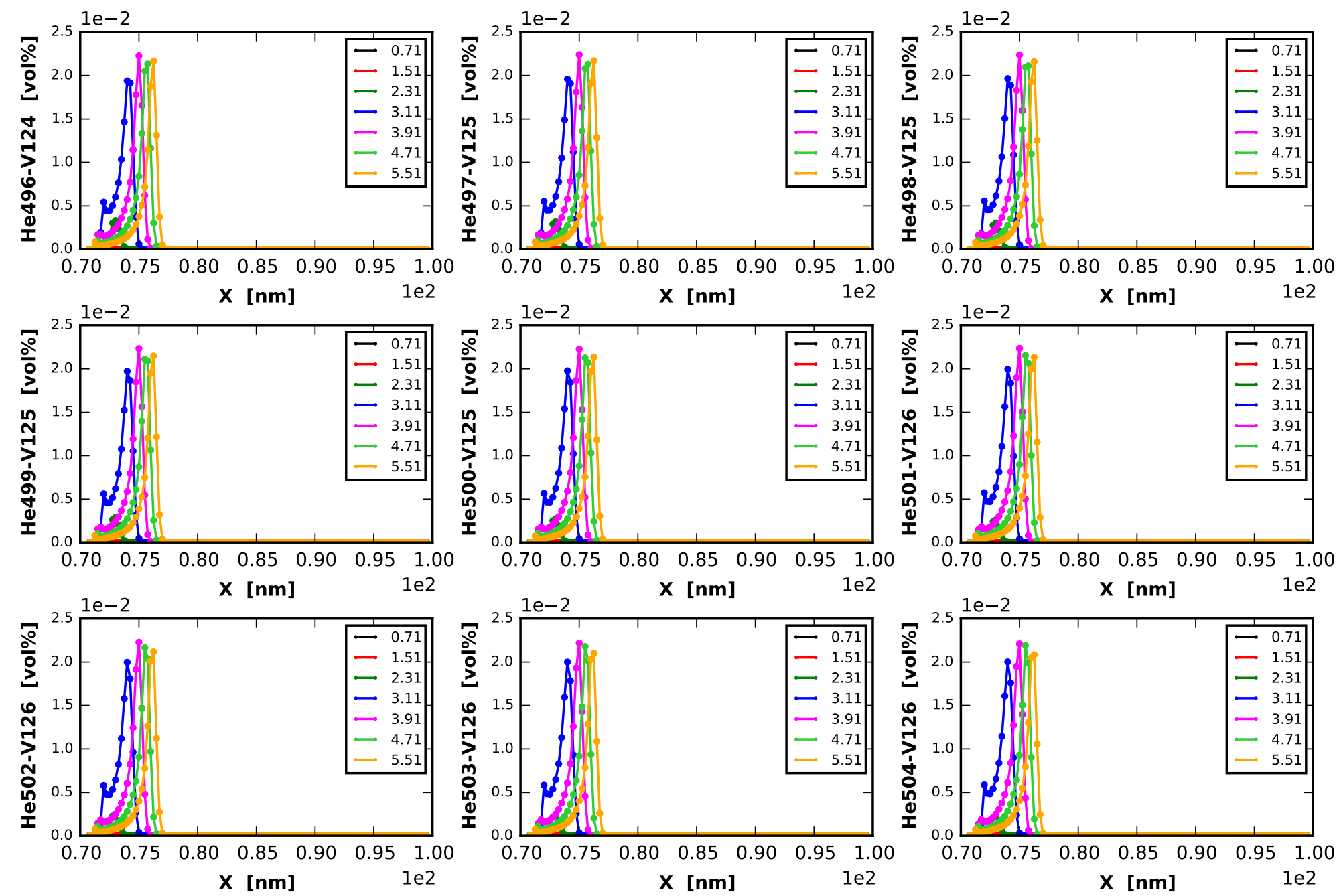
Transient: $[0.71,1.51,2.31,3.11,3.91,4.71,5.51]$ [us]
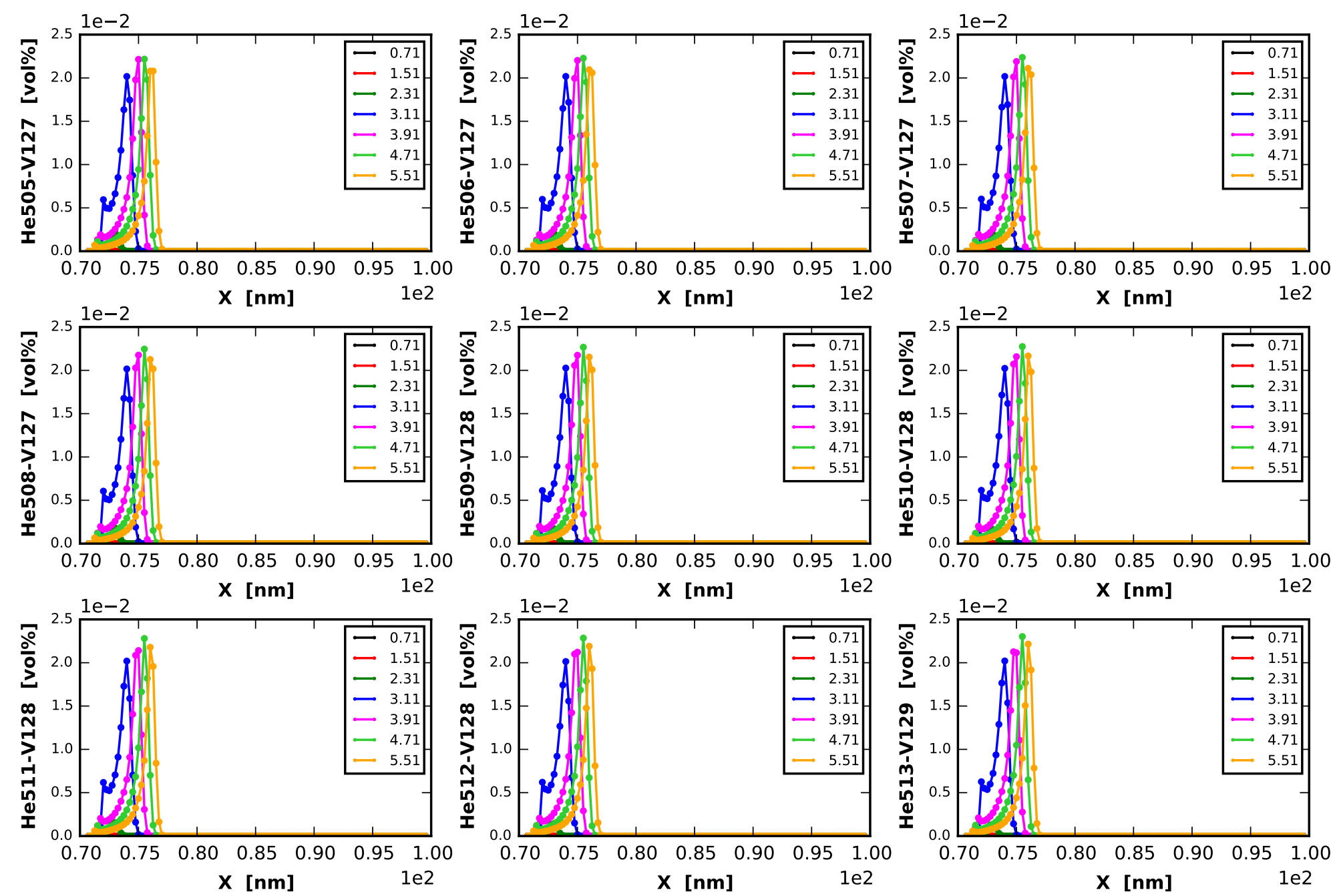
Transient: $[0.71,1.51,2.31,3.11,3.91,4.71,5.51]$ [us]
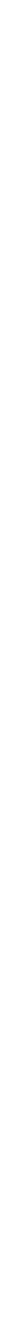
Transient: $[0.71,1.51,2.31,3.11,3.91,4.71,5.51]$ [us]
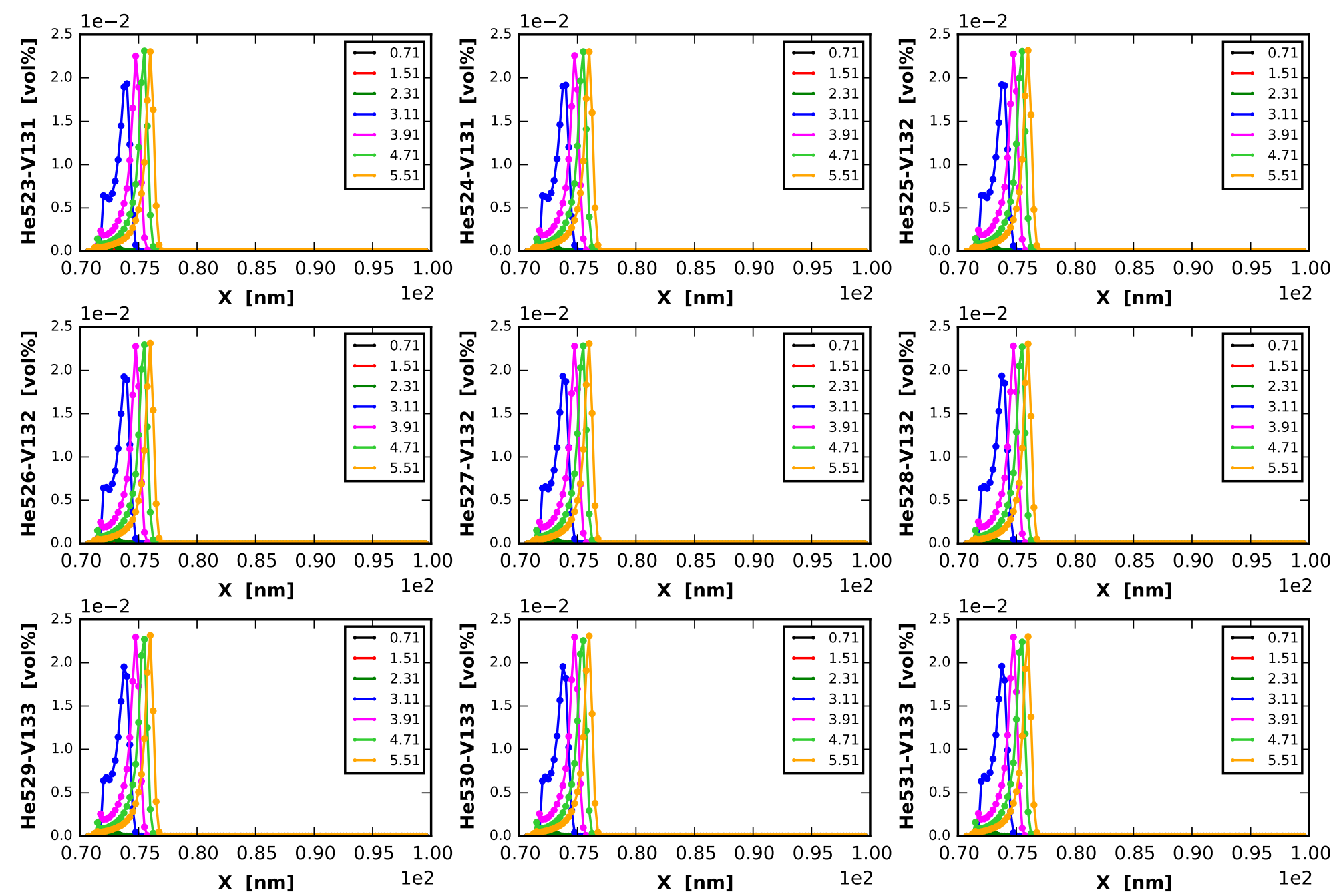
Transient: $[0.71,1.51,2.31,3.11,3.91,4.71,5.51]$ [us]
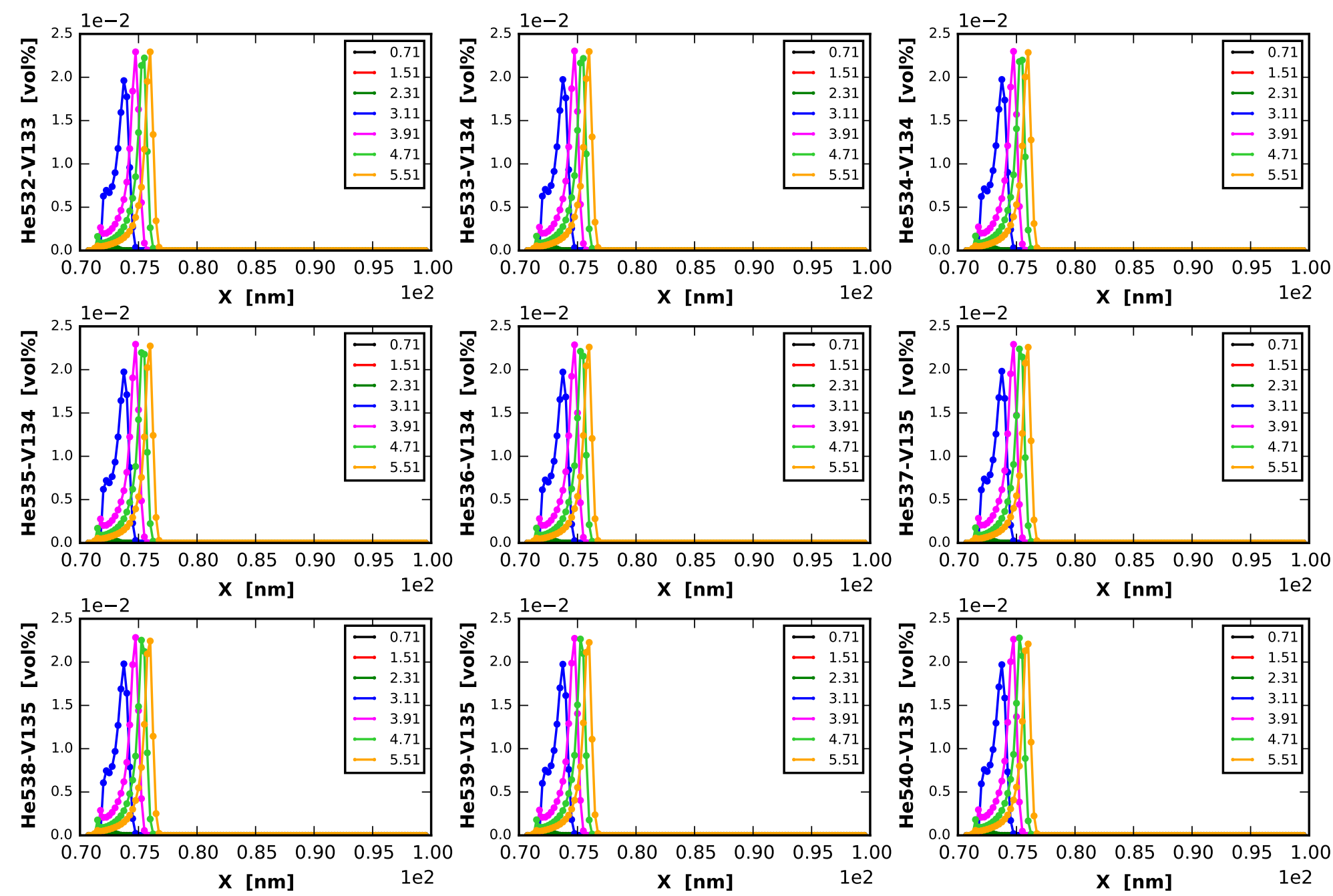
Transient: $[0.71,1.51,2.31,3.11,3.91,4.71,5.51]$ [us]
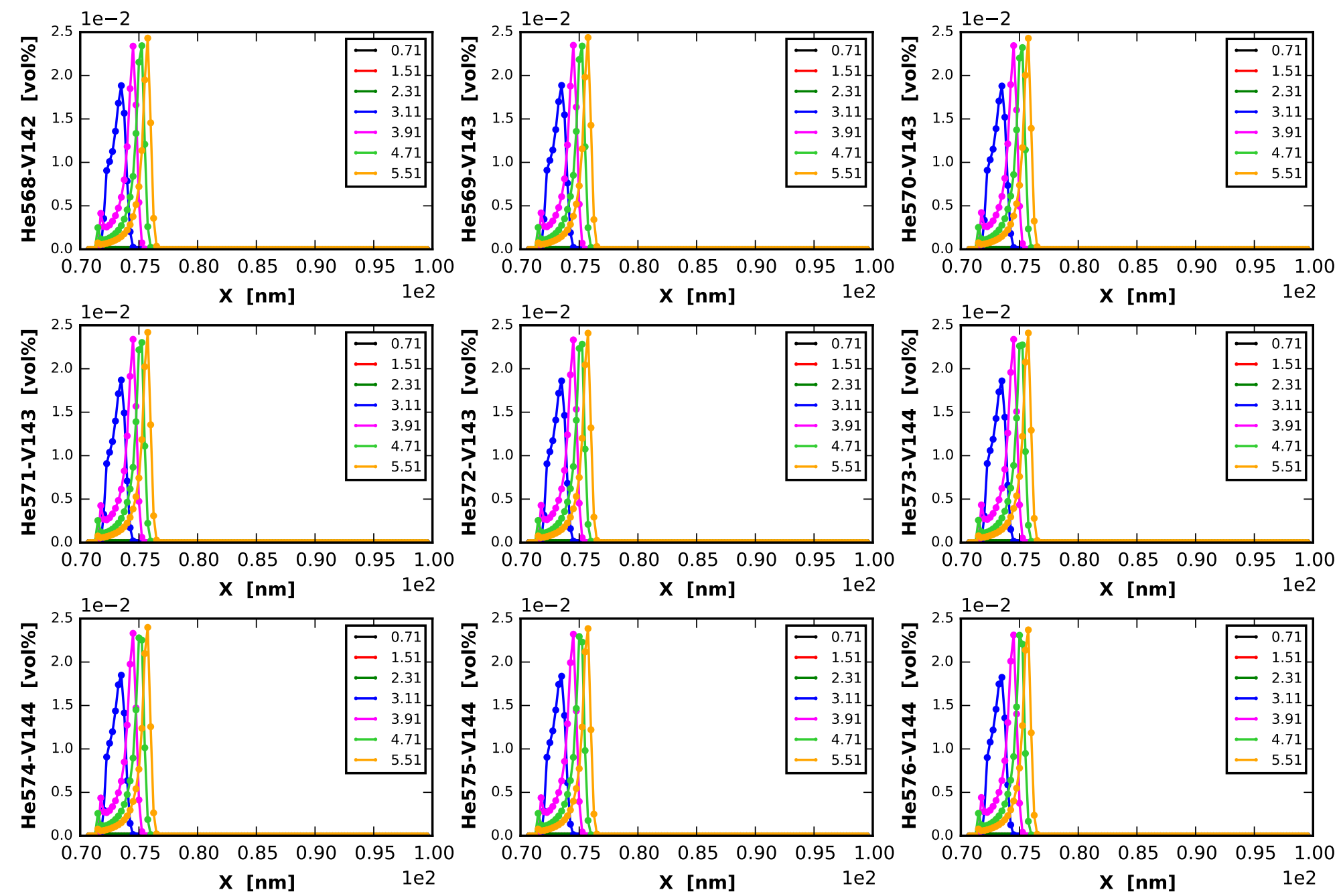
Transient: $[0.71,1.51,2.31,3.11,3.91,4.71,5.51]$ [us]
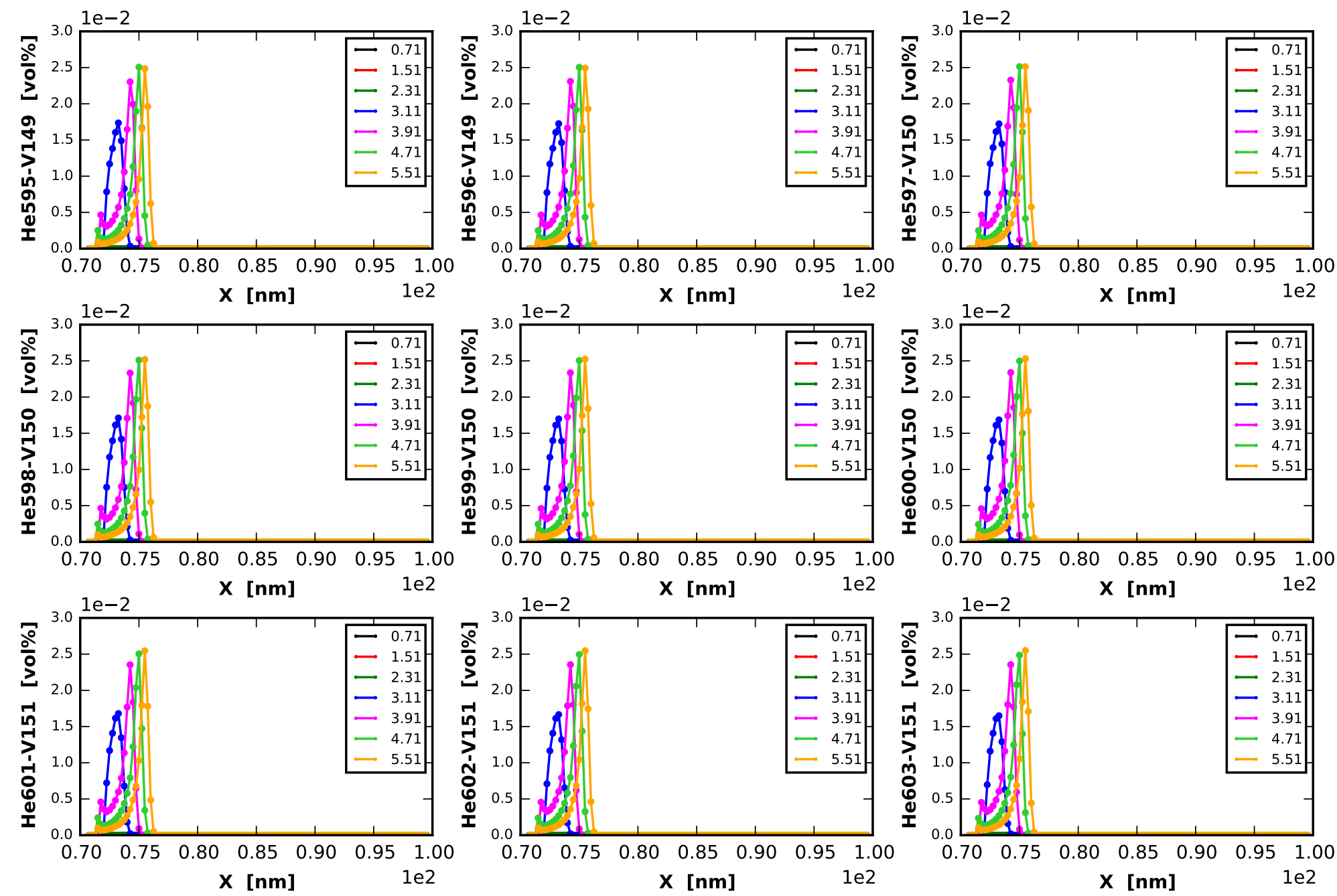
Transient: $[0.71,1.51,2.31,3.11,3.91,4.71,5.51]$ [us]
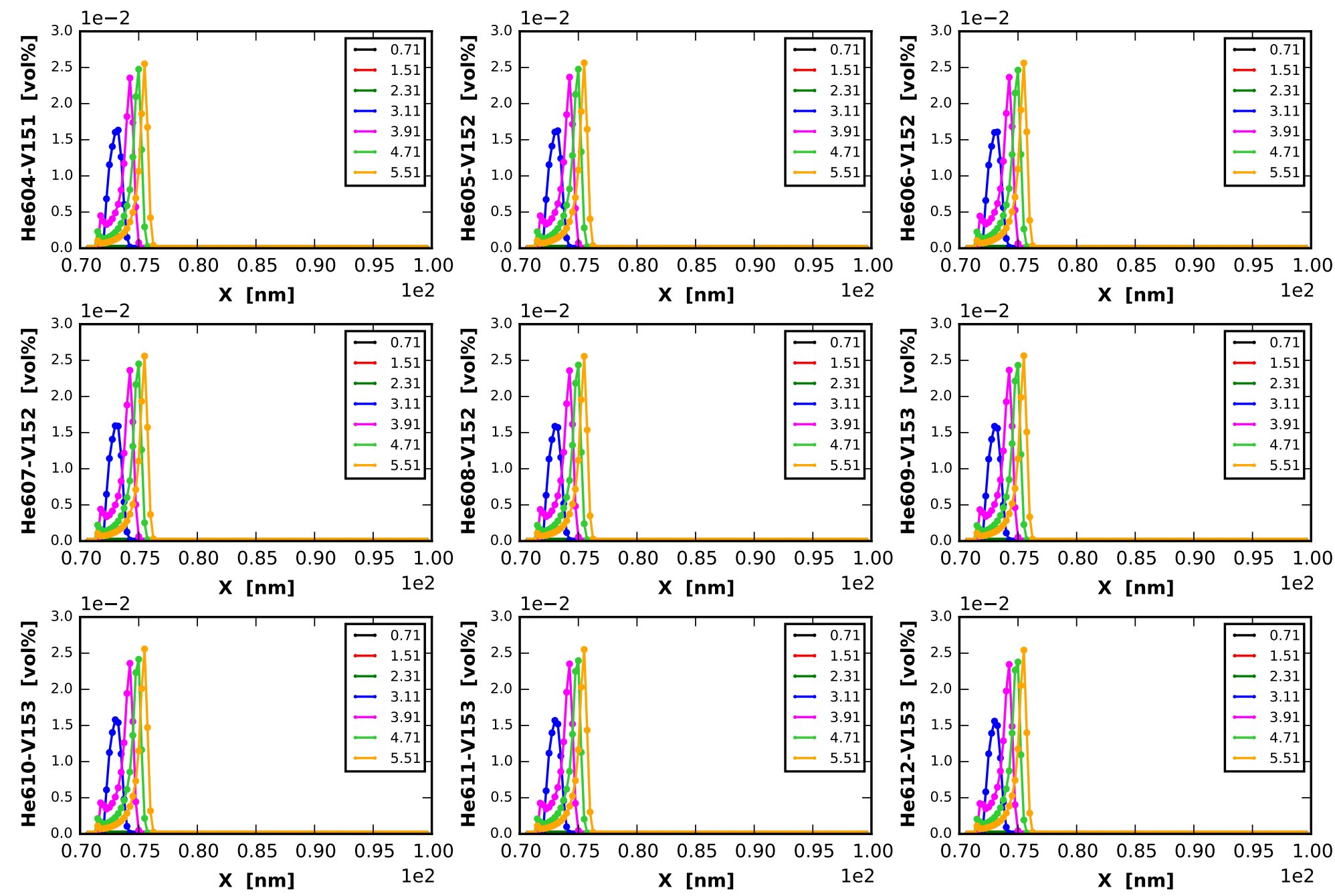
Transient: $[0.71,1.51,2.31,3.11,3.91,4.71,5.51]$ [us]
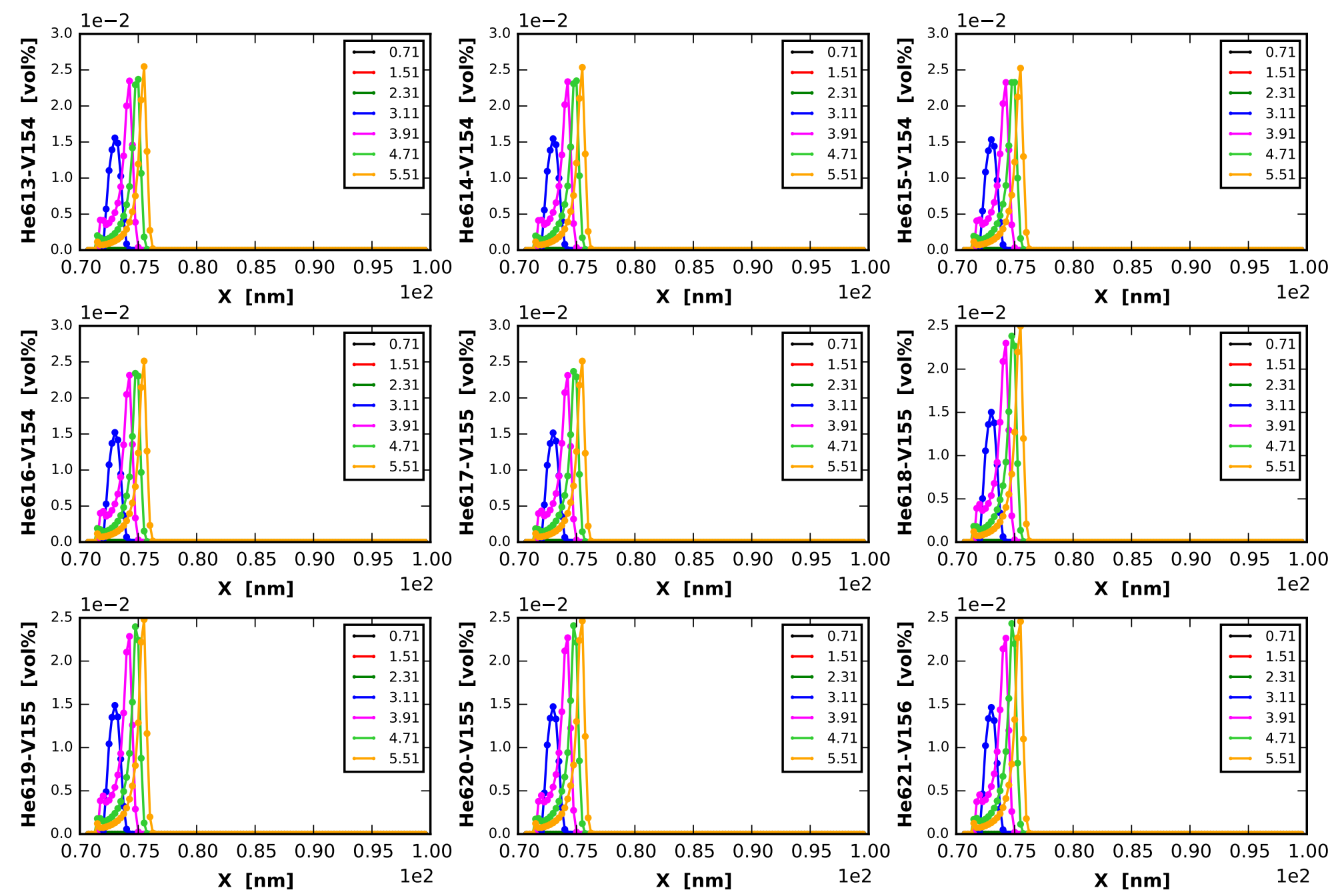
Transient: $[0.71,1.51,2.31,3.11,3.91,4.71,5.51]$ [us]
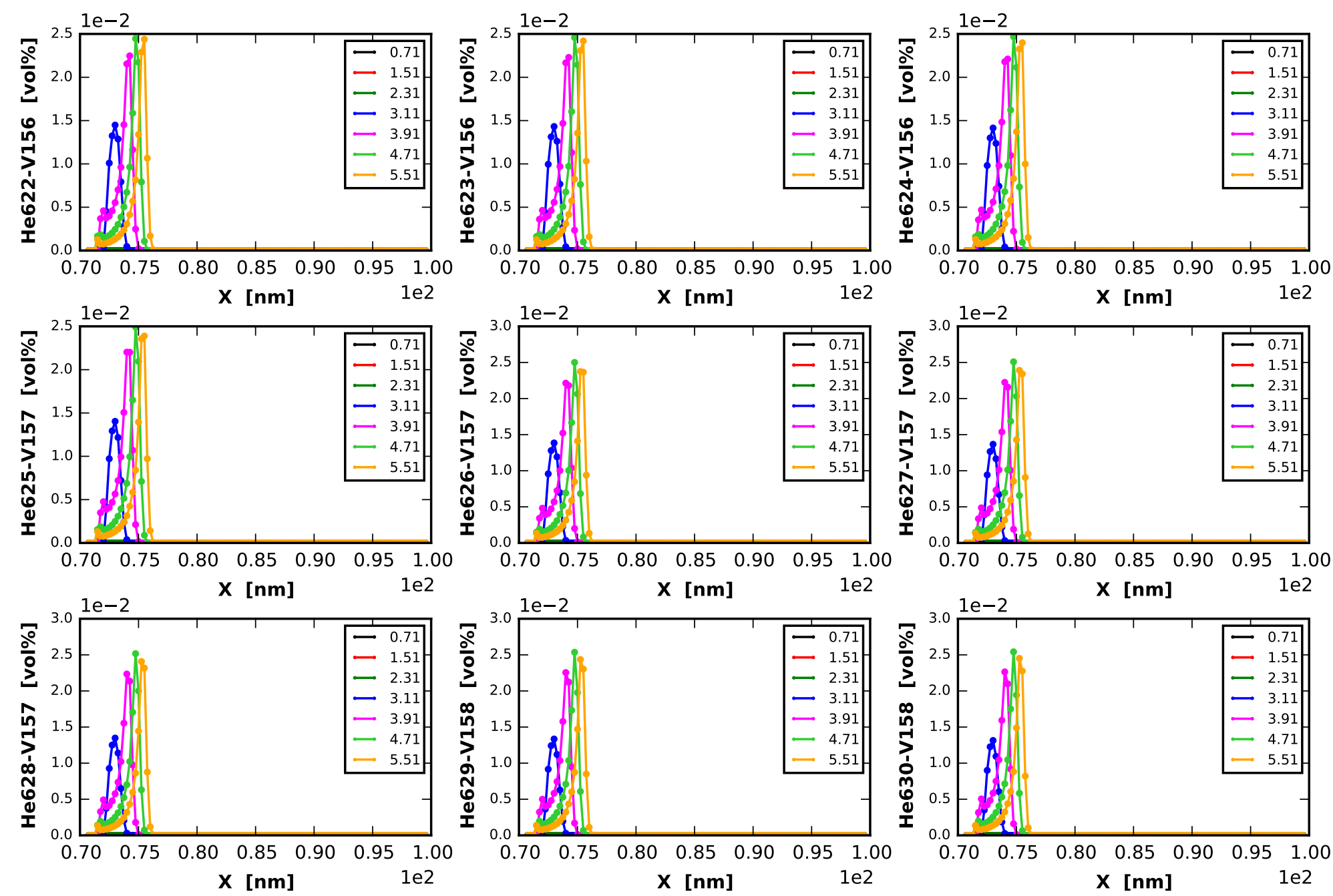
Transient: $[0.71,1.51,2.31,3.11,3.91,4.71,5.51]$ [us]
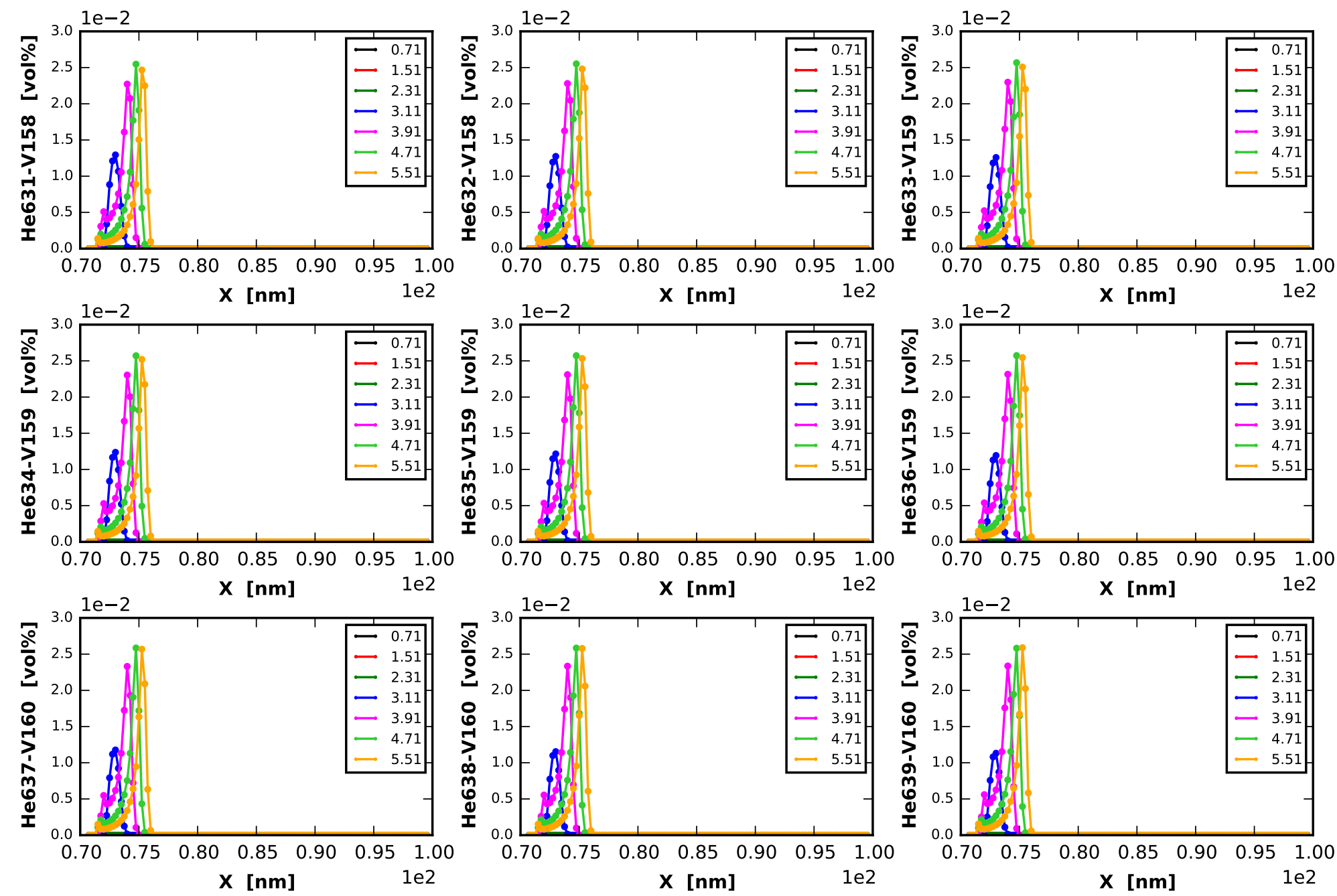
Transient: $[0.71,1.51,2.31,3.11,3.91,4.71,5.51]$ [us]
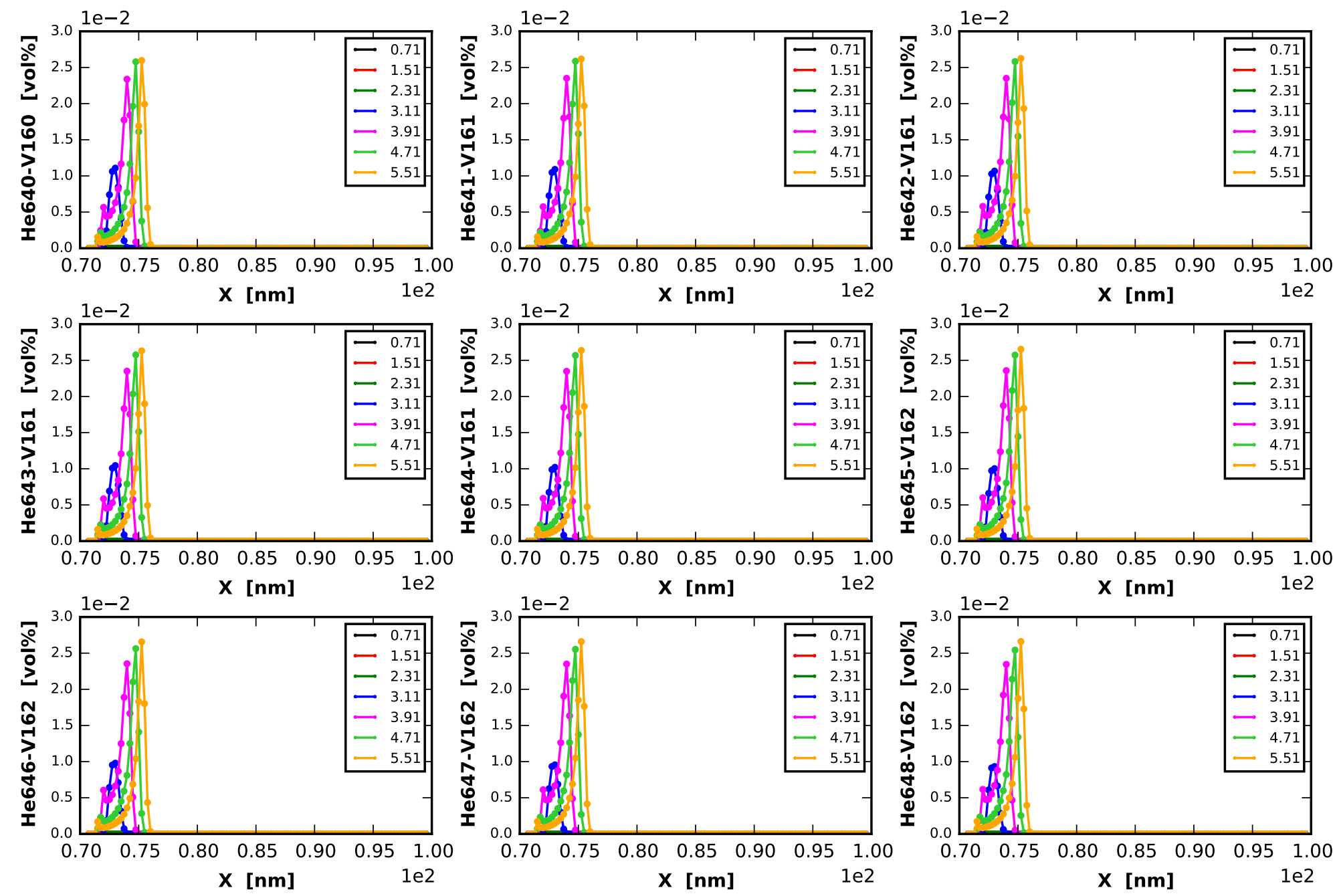
Transient: $[0.71,1.51,2.31,3.11,3.91,4.71,5.51]$ [us]
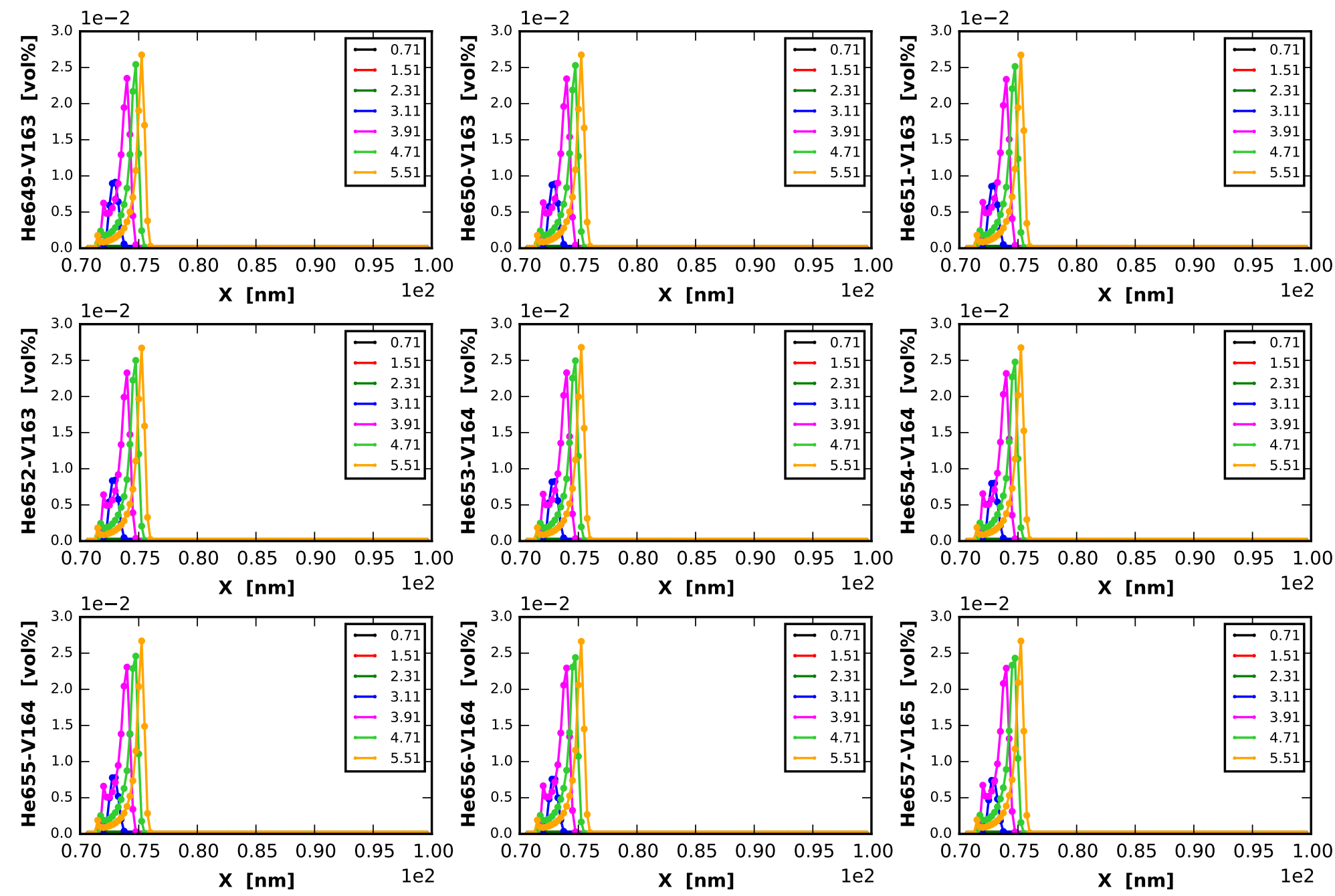
Transient: $[0.71,1.51,2.31,3.11,3.91,4.71,5.51]$ [us]
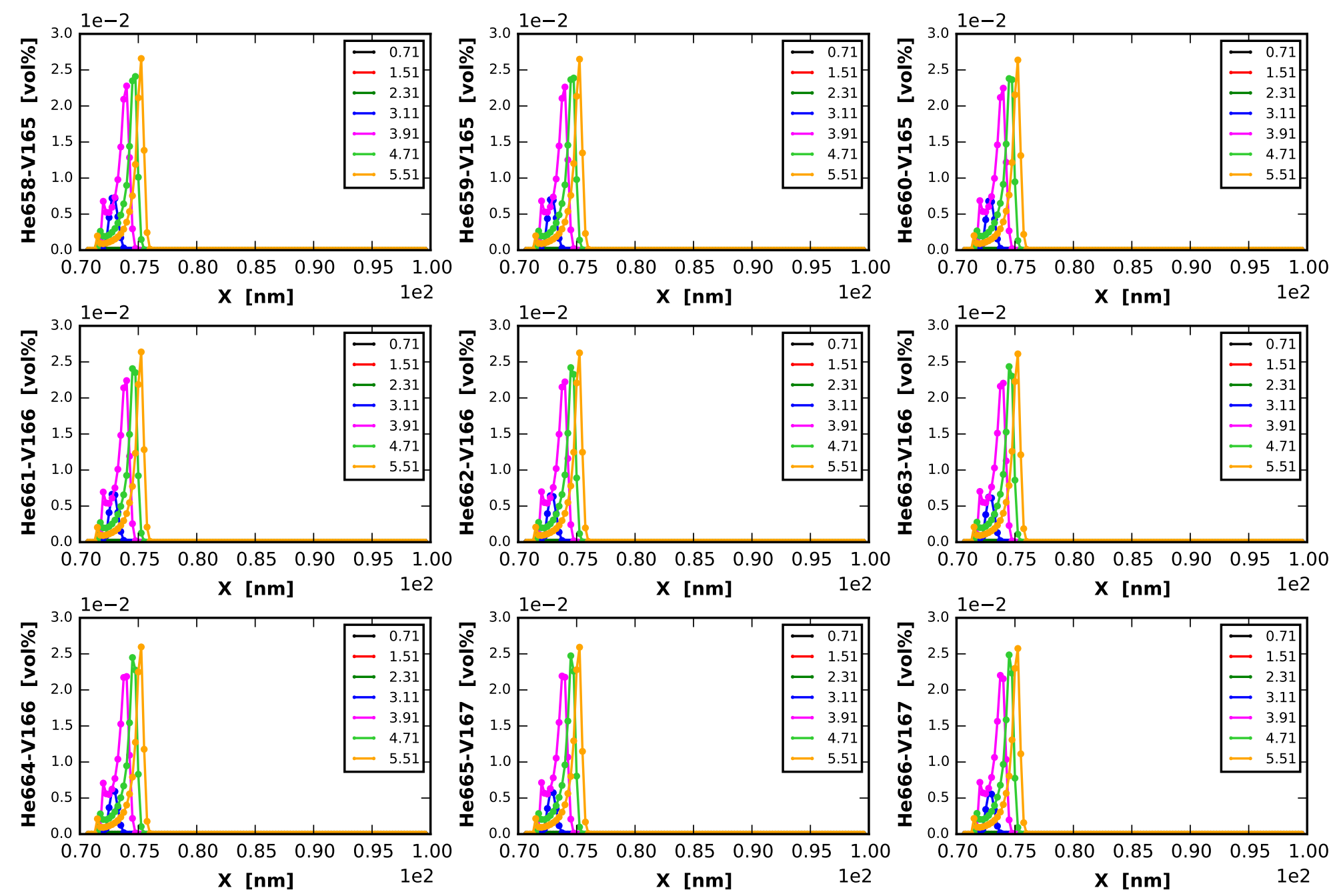
Transient: $[0.71,1.51,2.31,3.11,3.91,4.71,5.51]$ [us]
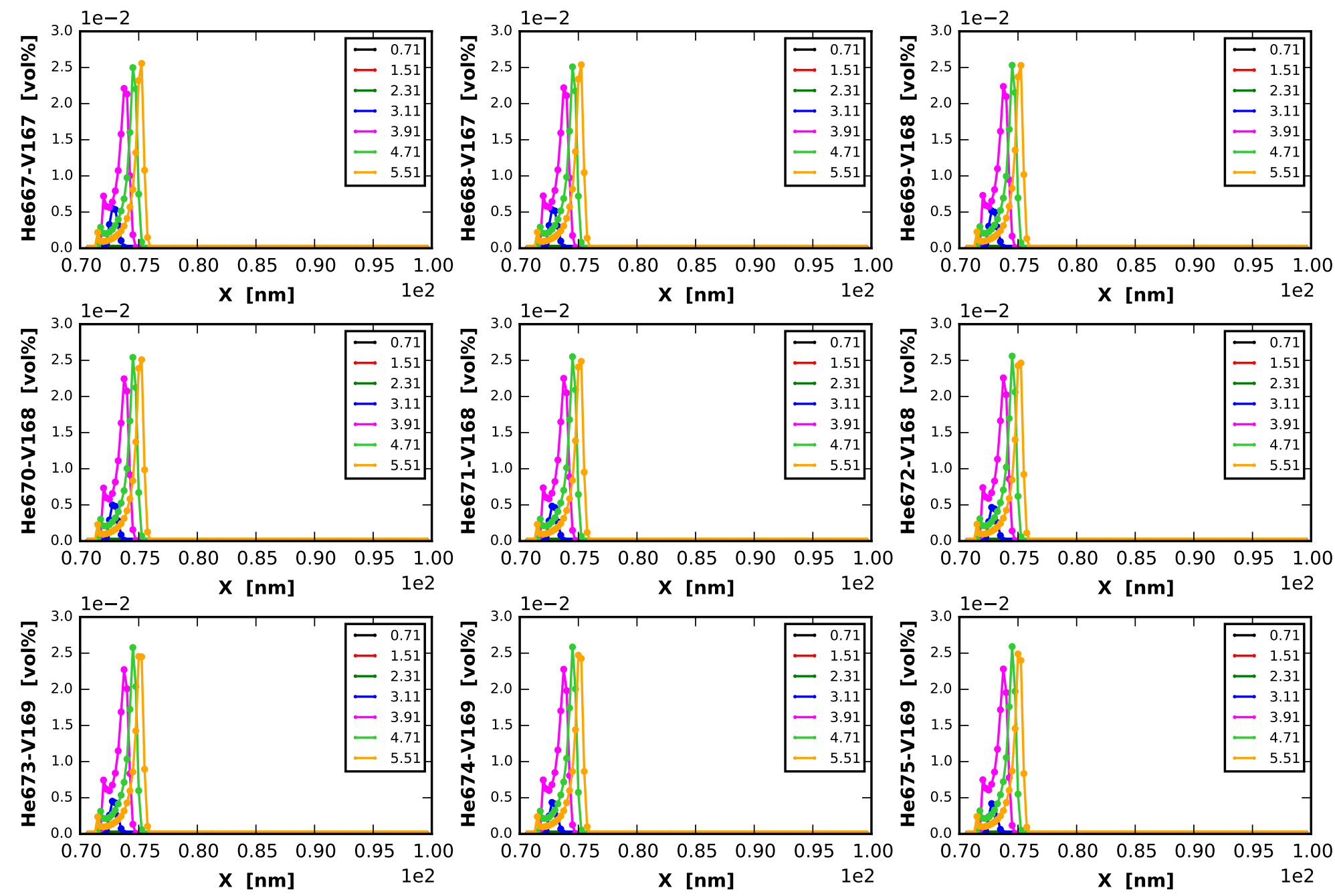
Transient: $[0.71,1.51,2.31,3.11,3.91,4.71,5.51]$ [us]
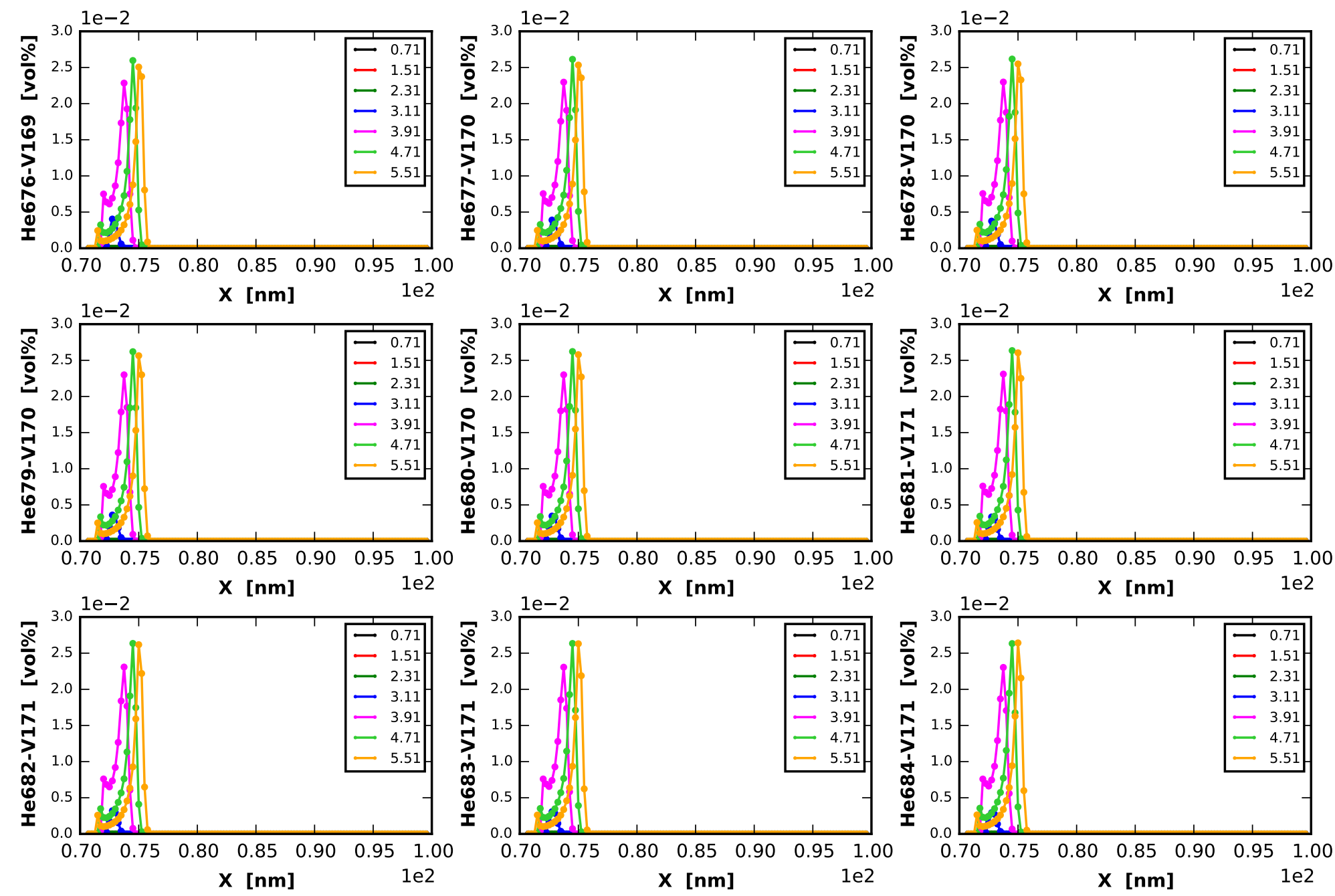
Transient: $[0.71,1.51,2.31,3.11,3.91,4.71,5.51]$ [us]
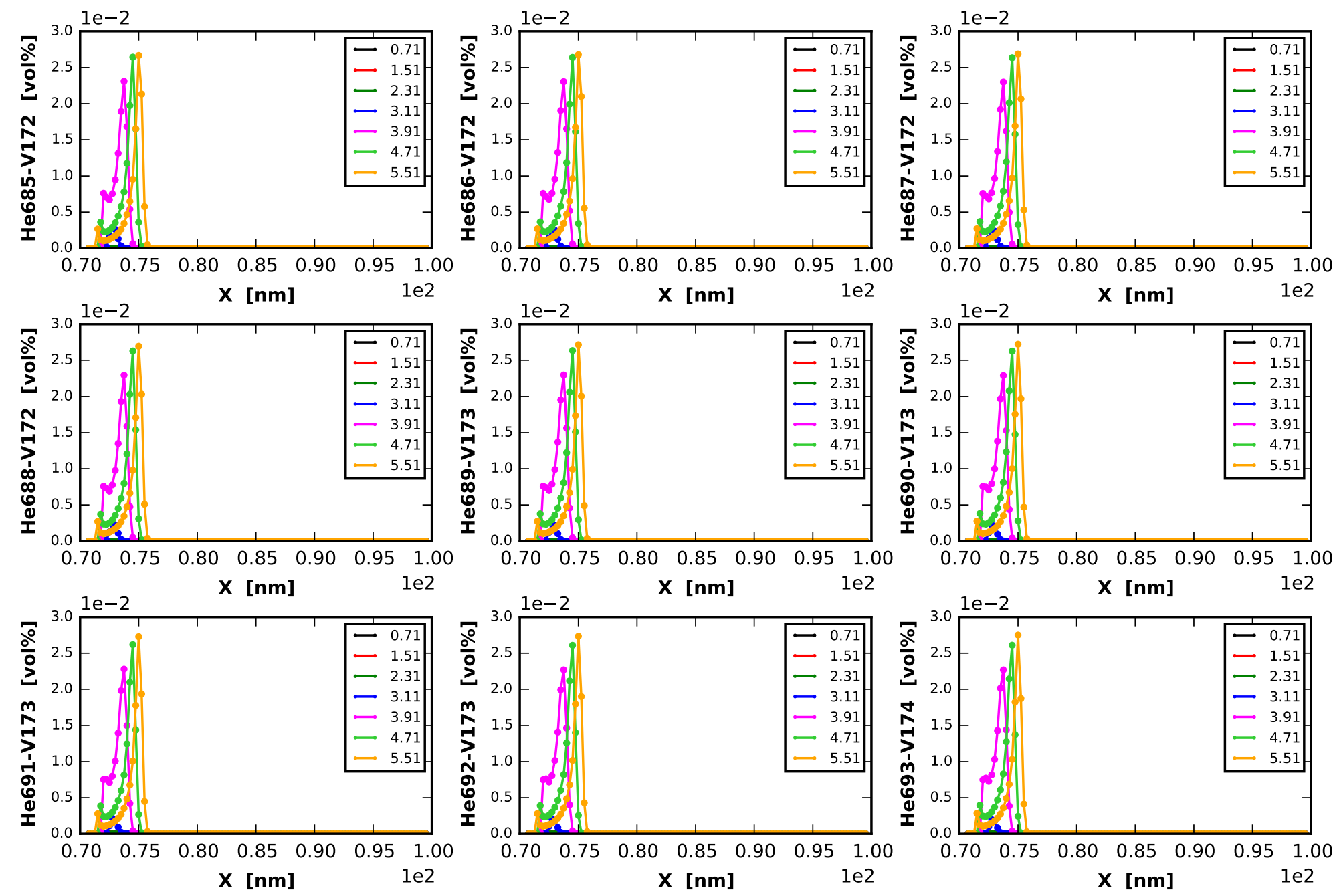
Transient: $[0.71,1.51,2.31,3.11,3.91,4.71,5.51]$ [us]
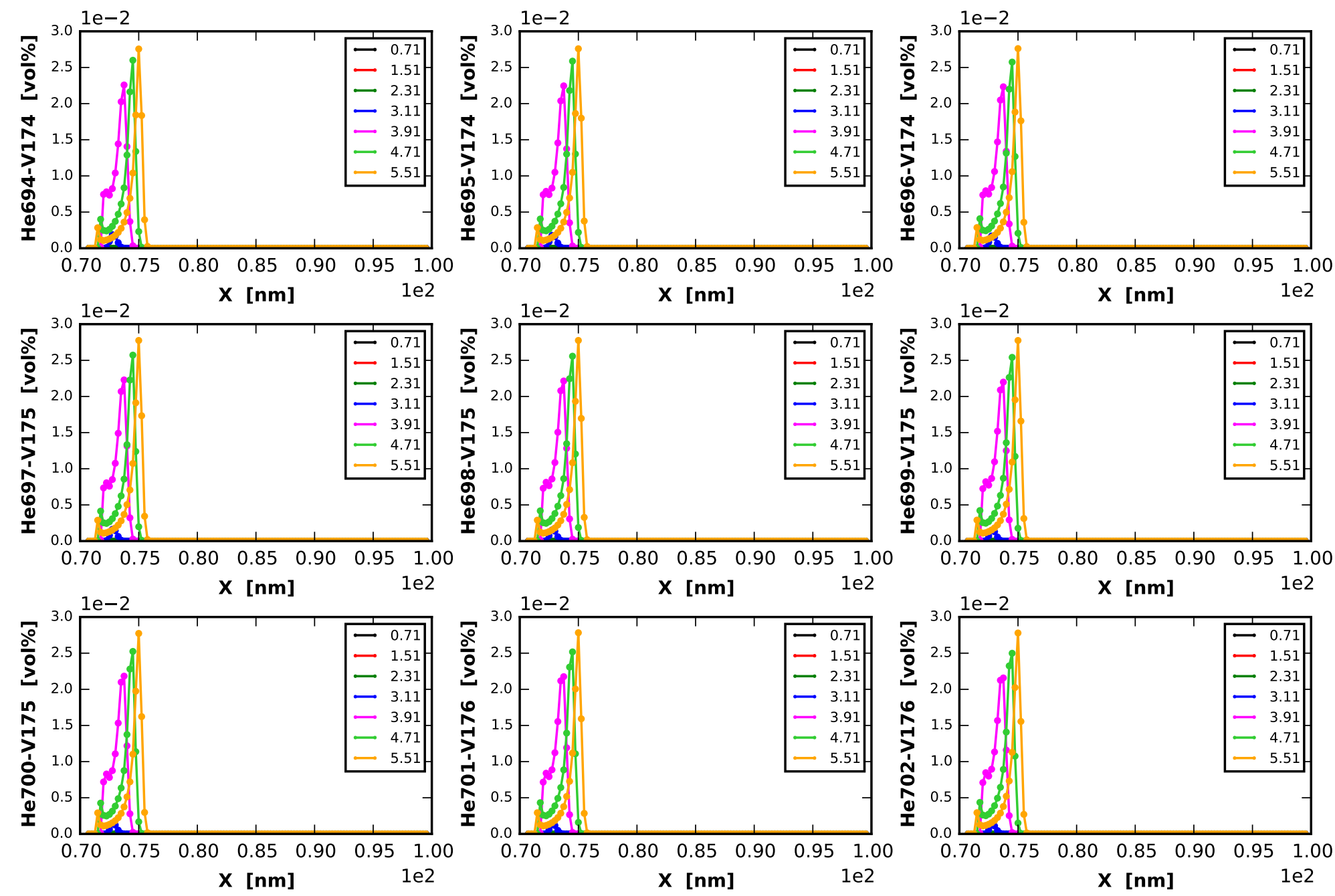
Transient: $[0.71,1.51,2.31,3.11,3.91,4.71,5.51]$ [us]
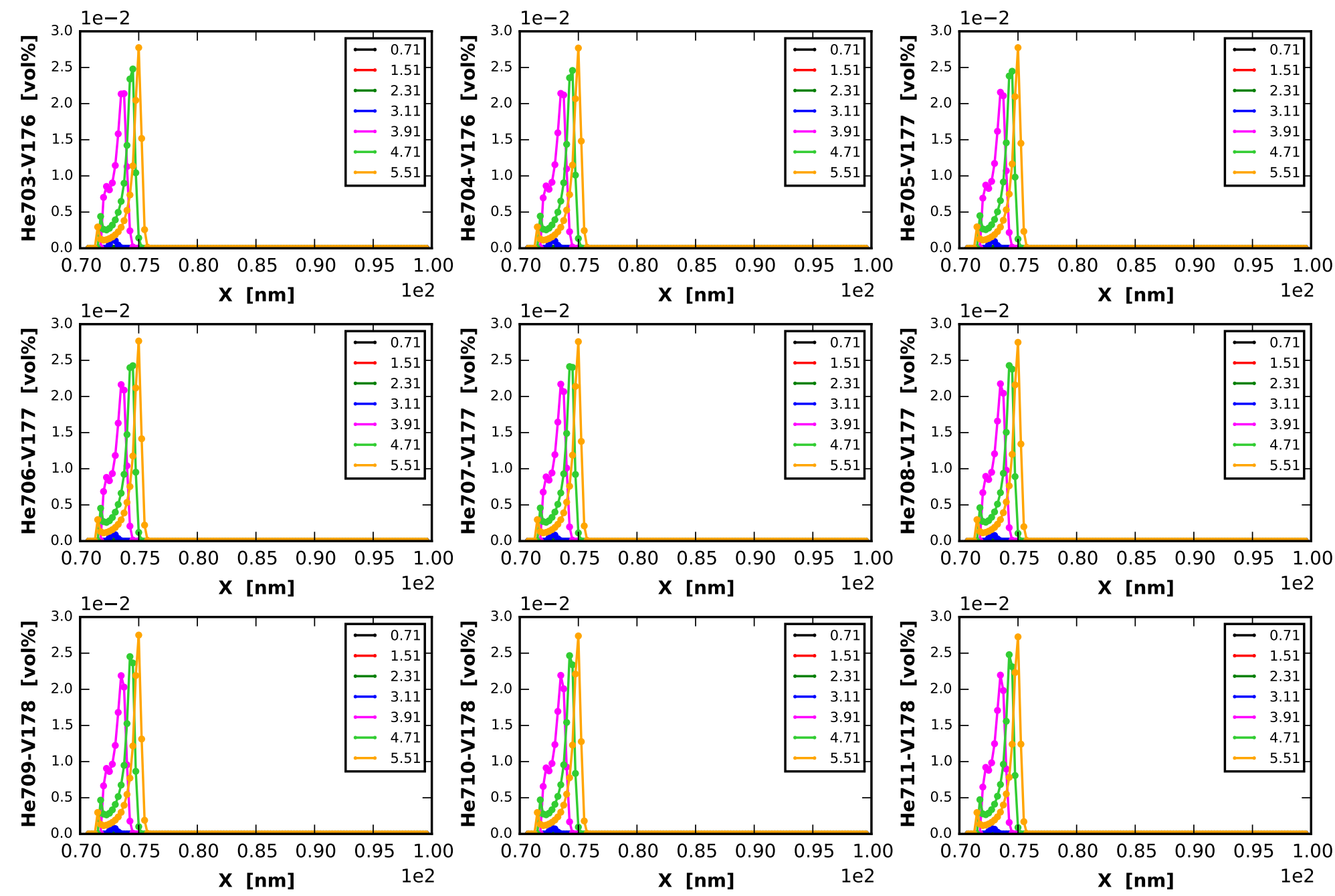
Transient: $[0.71,1.51,2.31,3.11,3.91,4.71,5.51]$ [us]
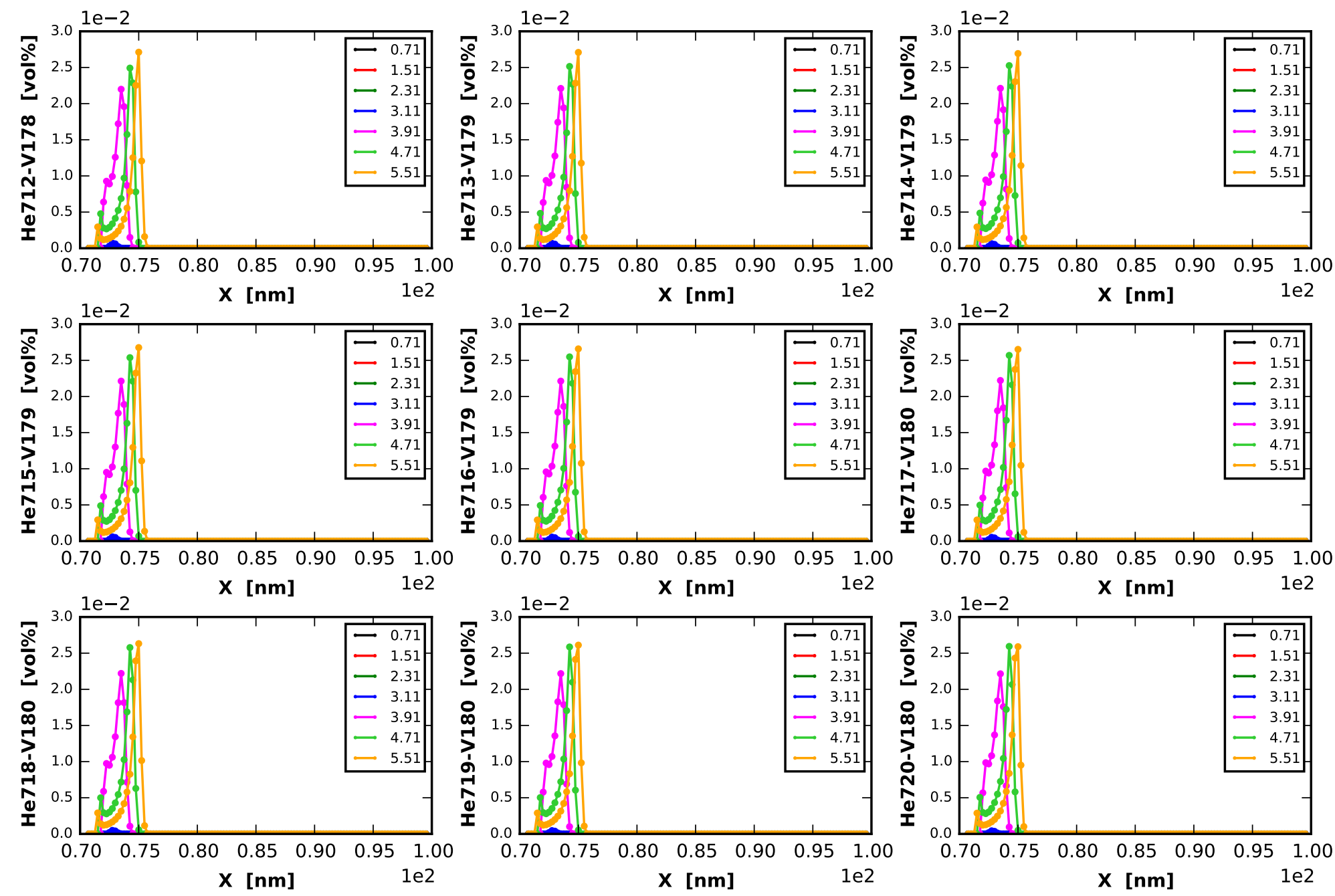
Transient: $[0.71,1.51,2.31,3.11,3.91,4.71,5.51]$ [us]
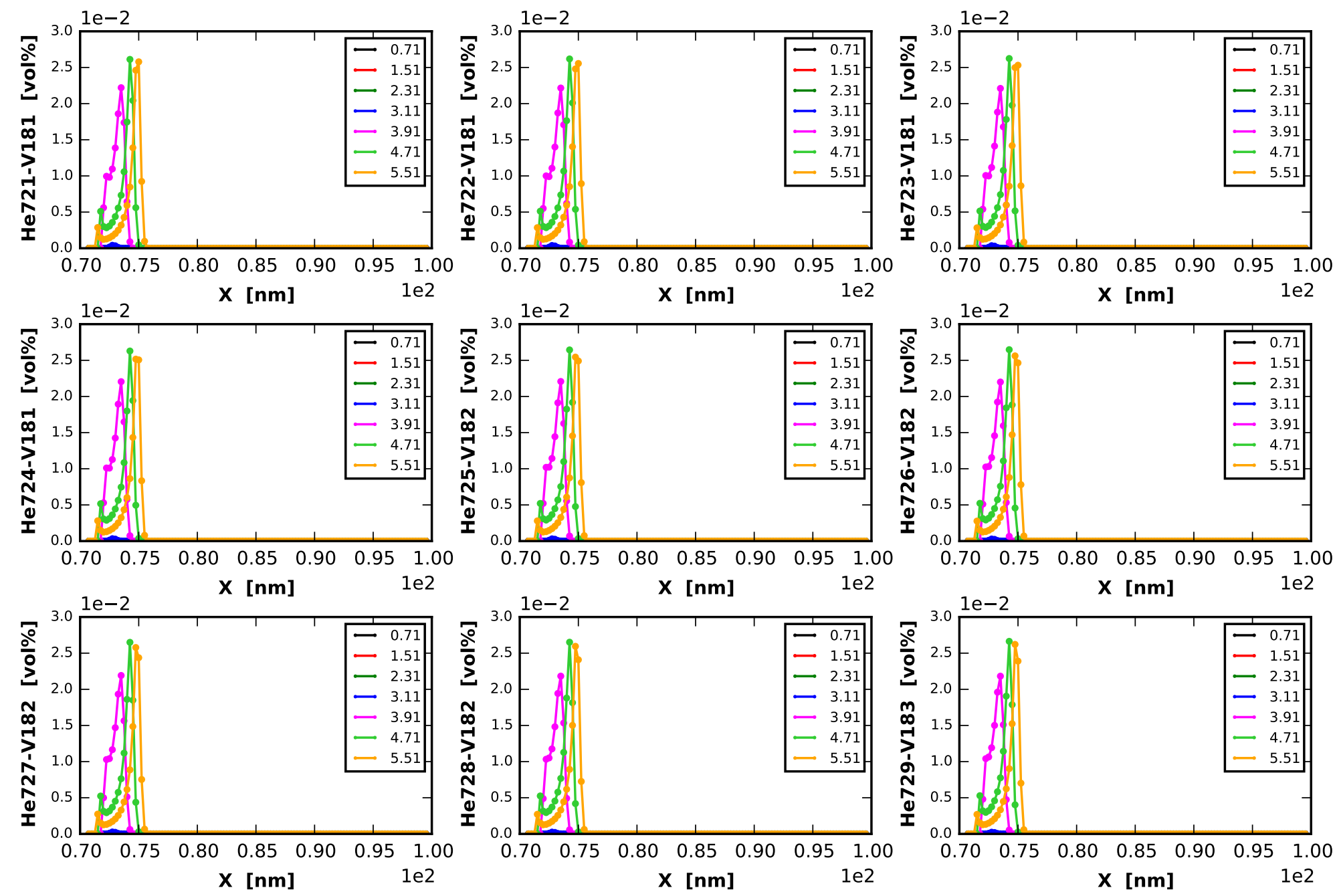
Transient: $[0.71,1.51,2.31,3.11,3.91,4.71,5.51]$ [us]
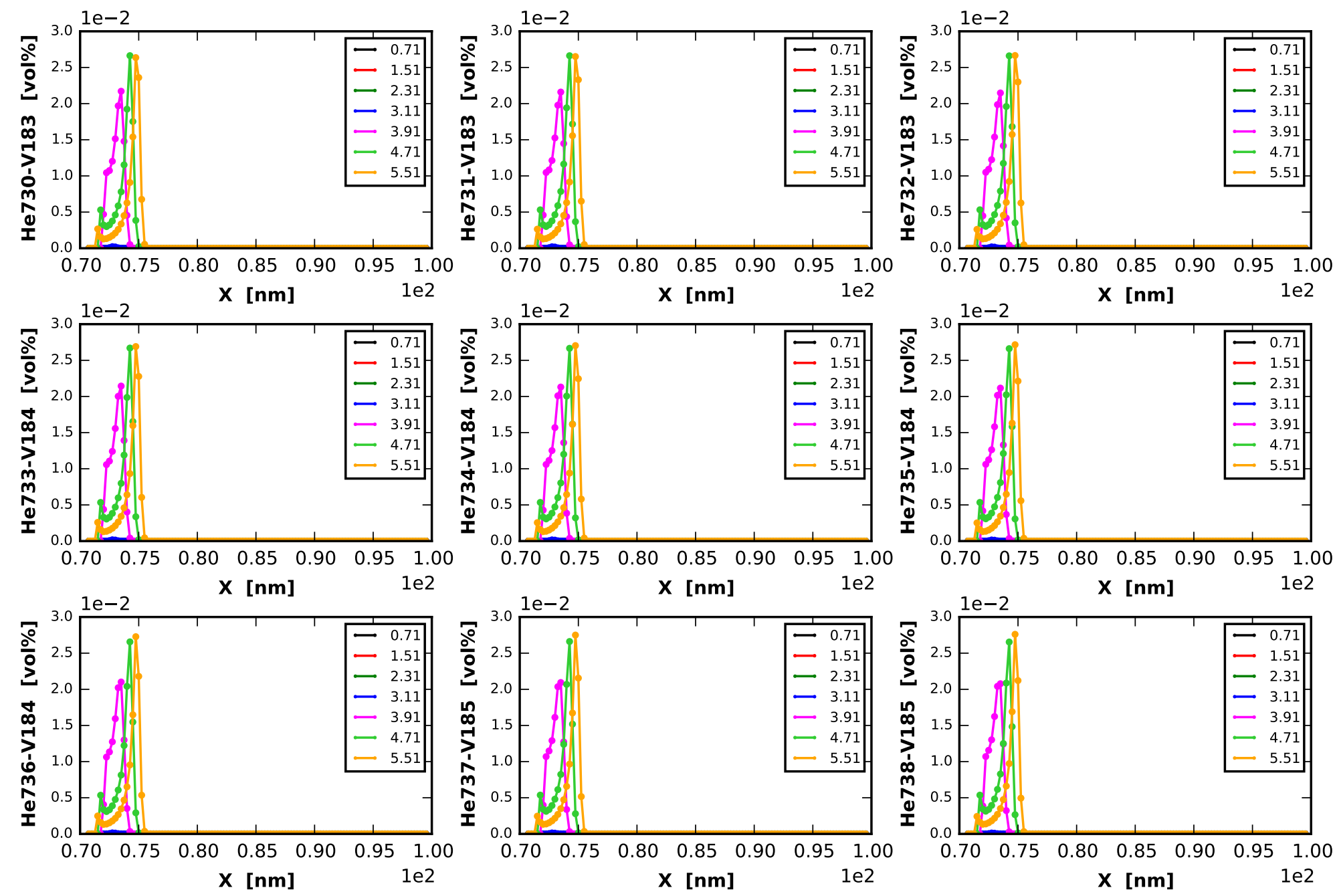
Transient: $[0.71,1.51,2.31,3.11,3.91,4.71,5.51]$ [us]
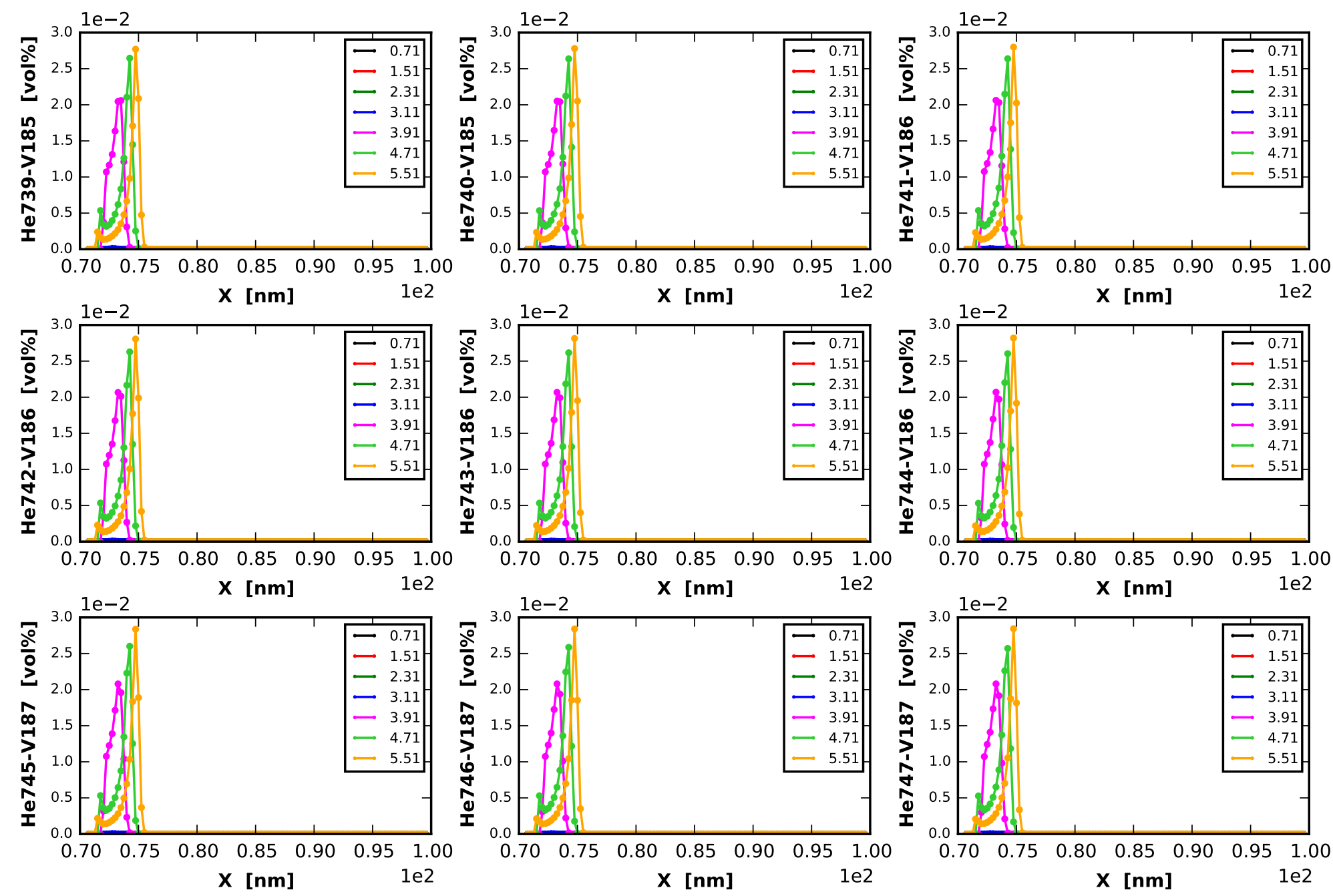
Transient: $[0.71,1.51,2.31,3.11,3.91,4.71,5.51]$ [us]
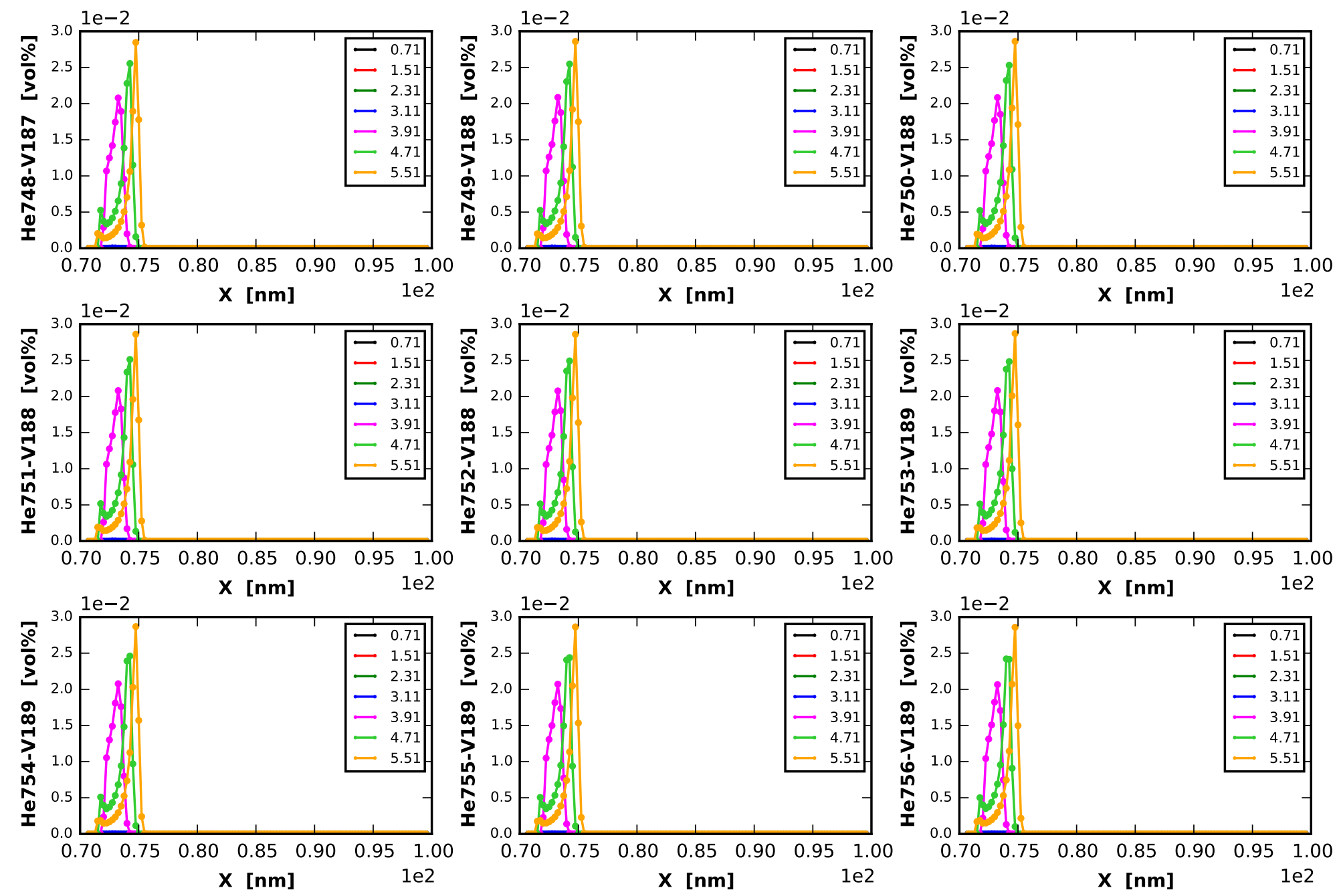


\section{Transient: [0.71, 1.51, 2.31, 3.11, 3.91, 4.71, 5.51] [us]}
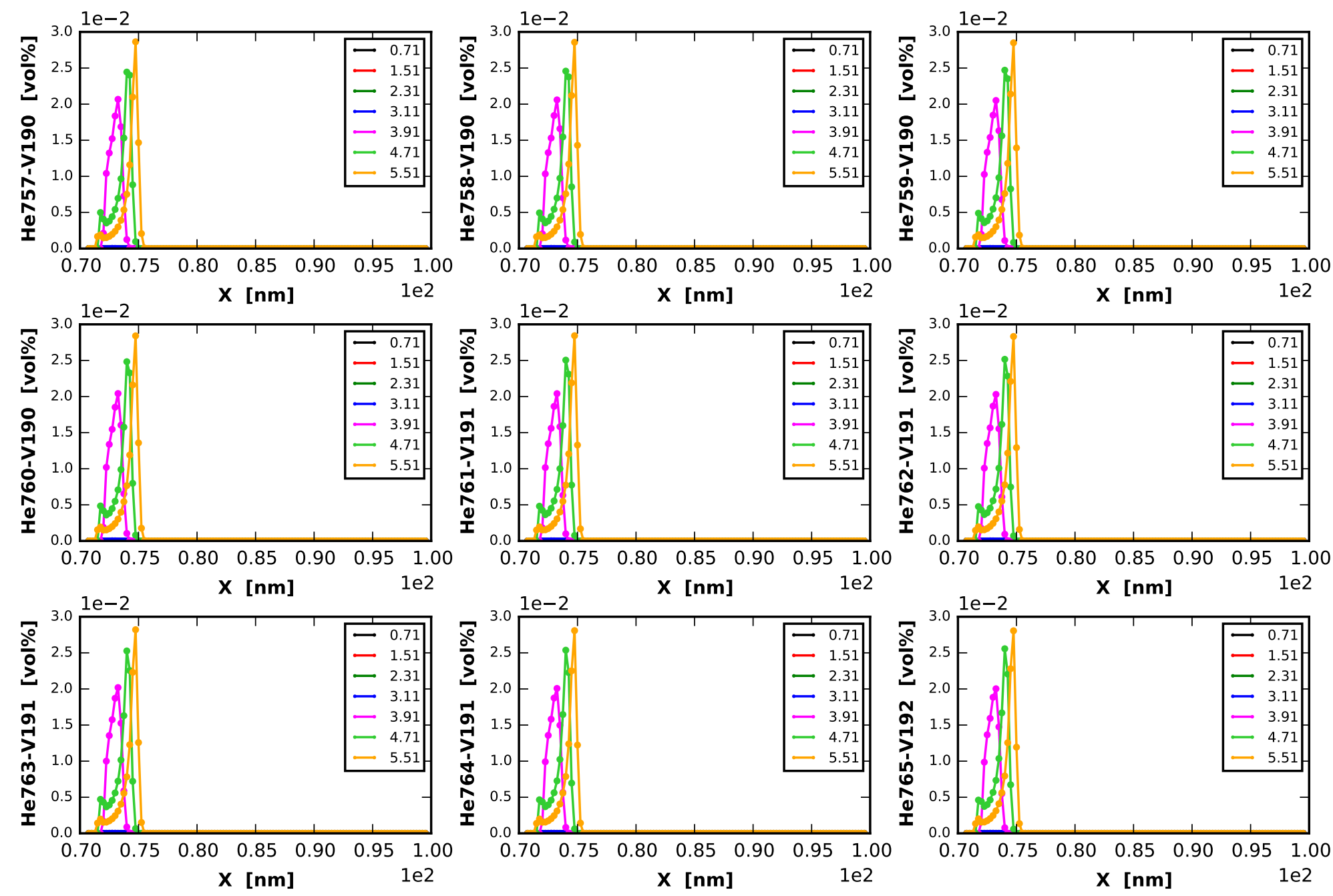
Transient: $[0.71,1.51,2.31,3.11,3.91,4.71,5.51]$ [us]
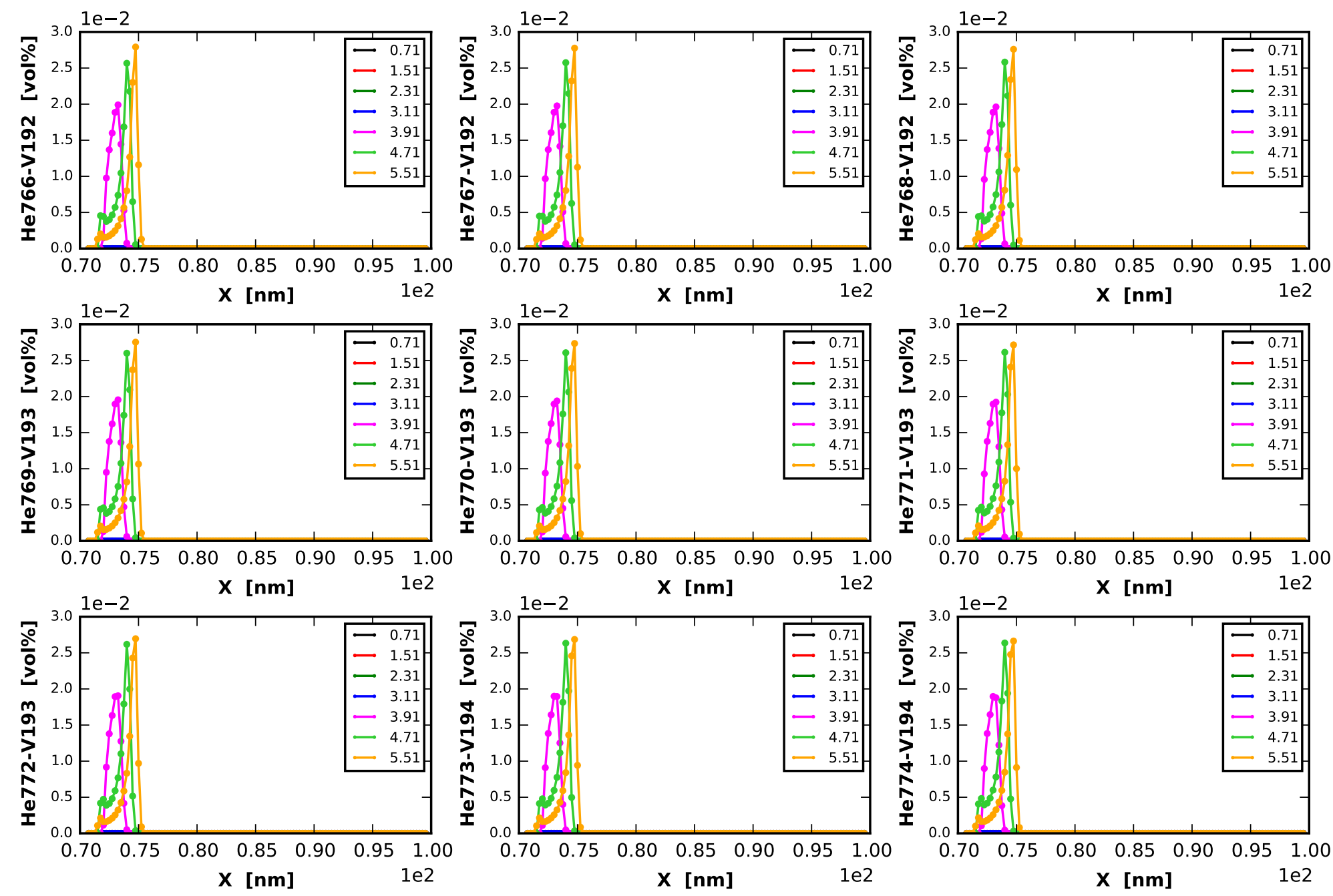


\section{Transient: [0.71, 1.51, 2.31, 3.11, 3.91, 4.71, 5.51] [us]}
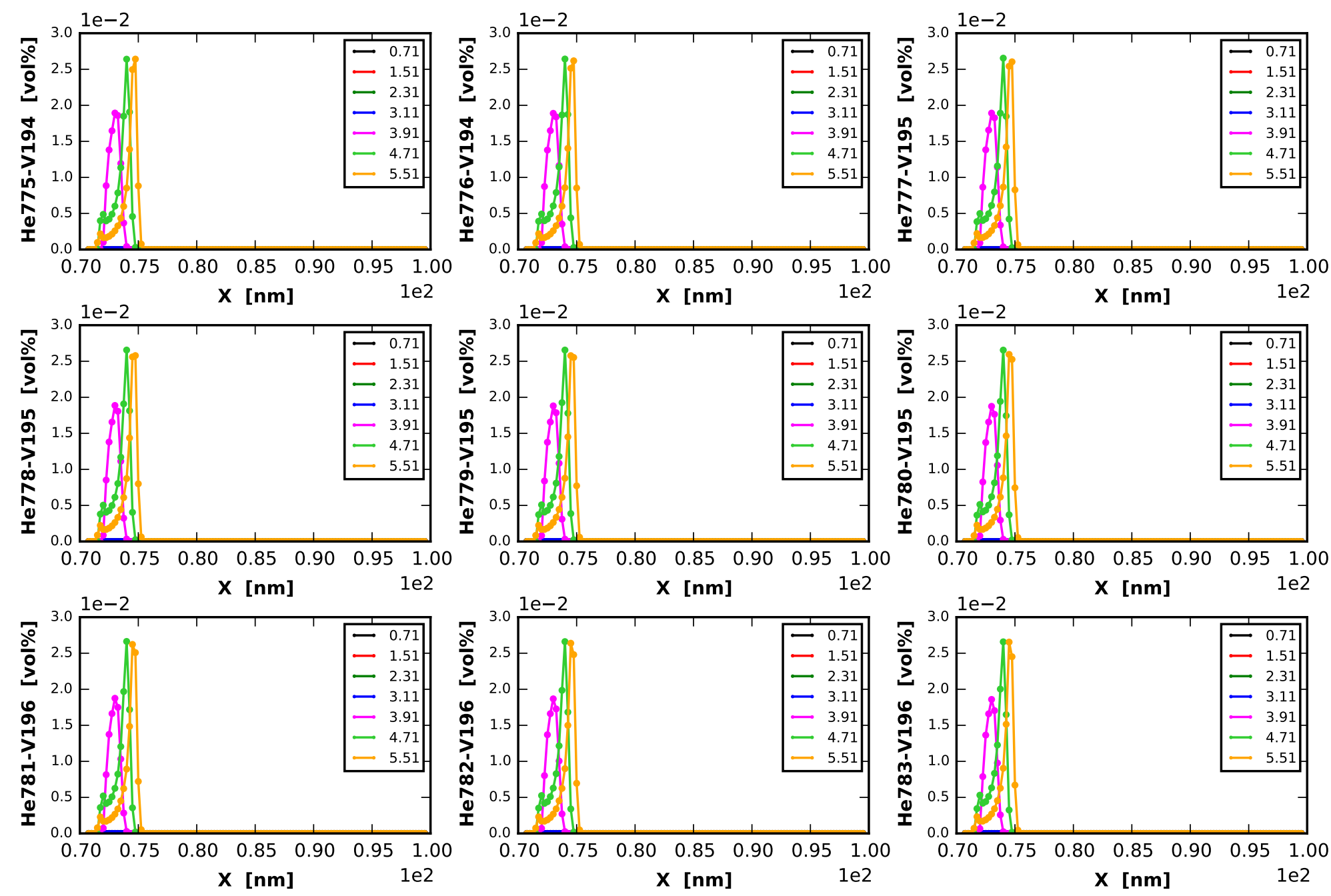
Transient: $[0.71,1.51,2.31,3.11,3.91,4.71,5.51]$ [us]
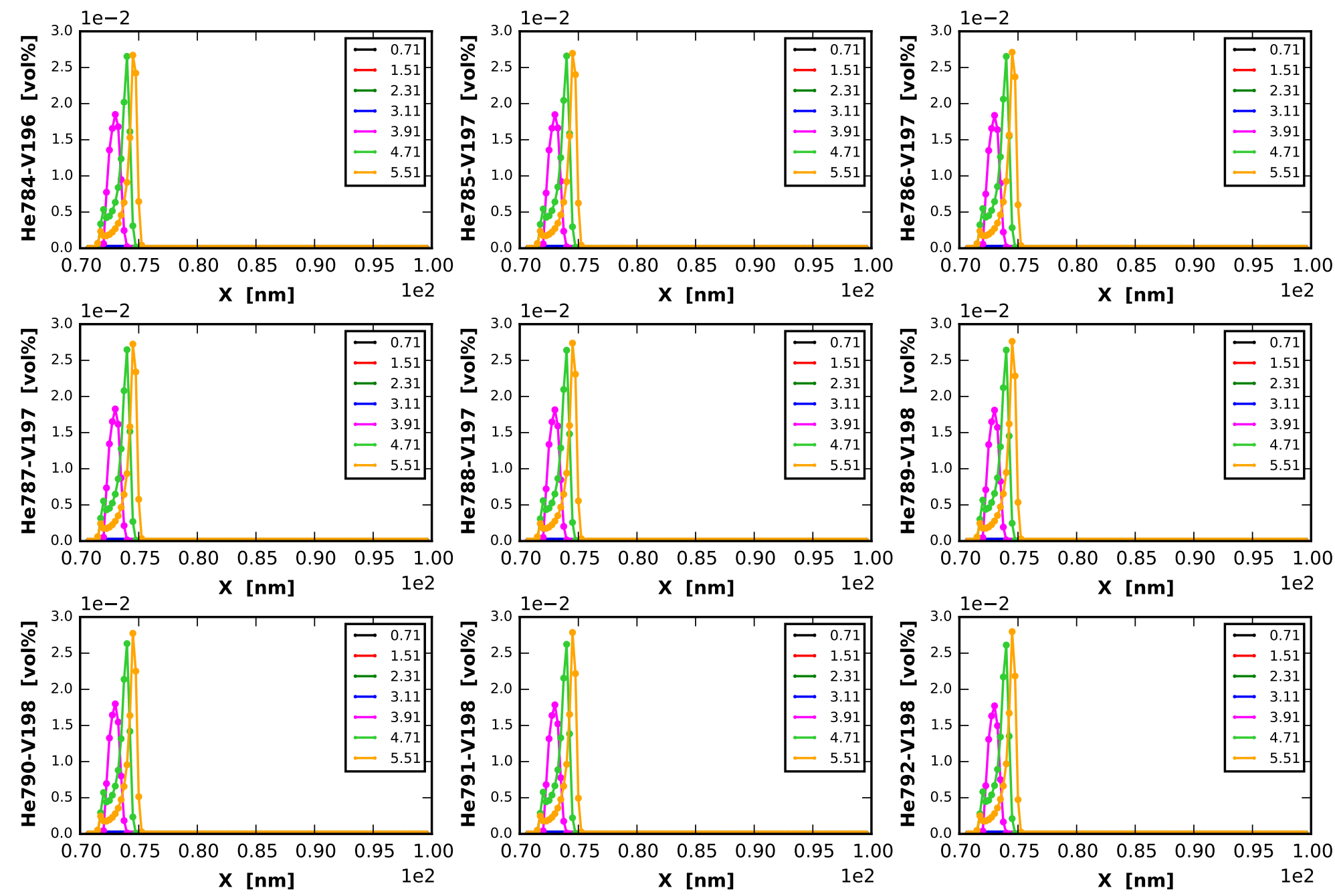
Transient: $[0.71,1.51,2.31,3.11,3.91,4.71,5.51]$ [us]
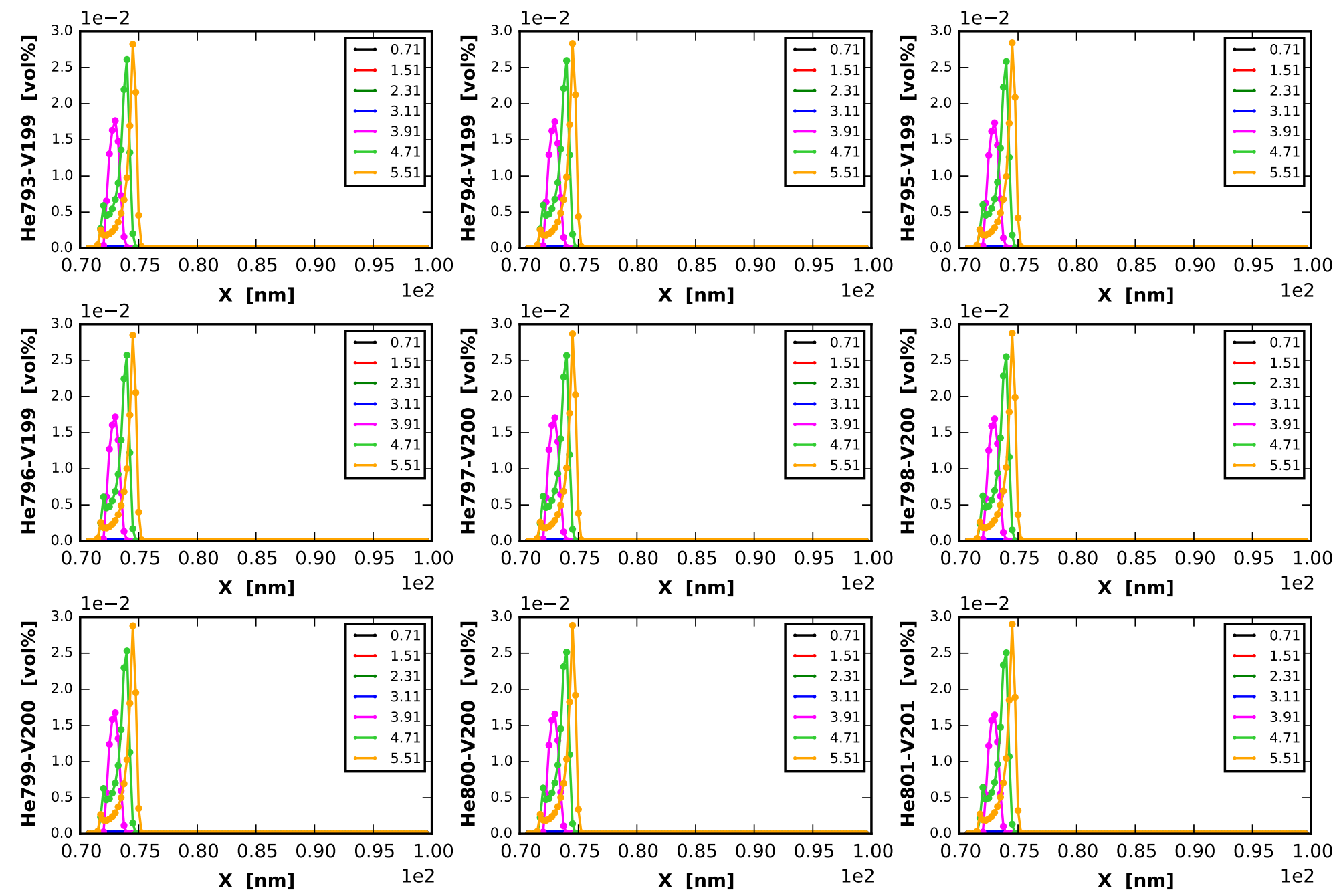
Transient: $[0.71,1.51,2.31,3.11,3.91,4.71,5.51]$ [us]
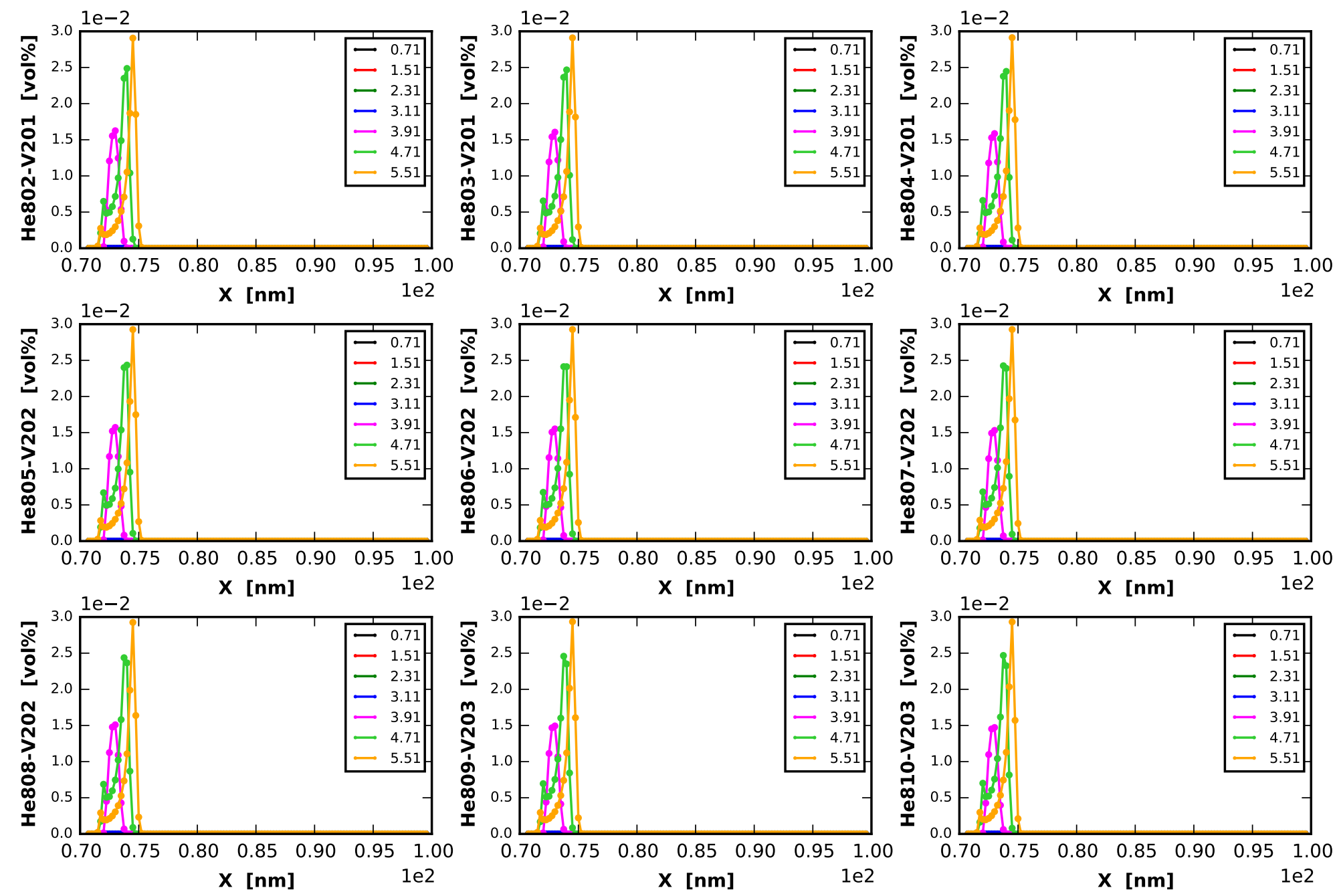
Transient: $[0.71,1.51,2.31,3.11,3.91,4.71,5.51]$ [us]
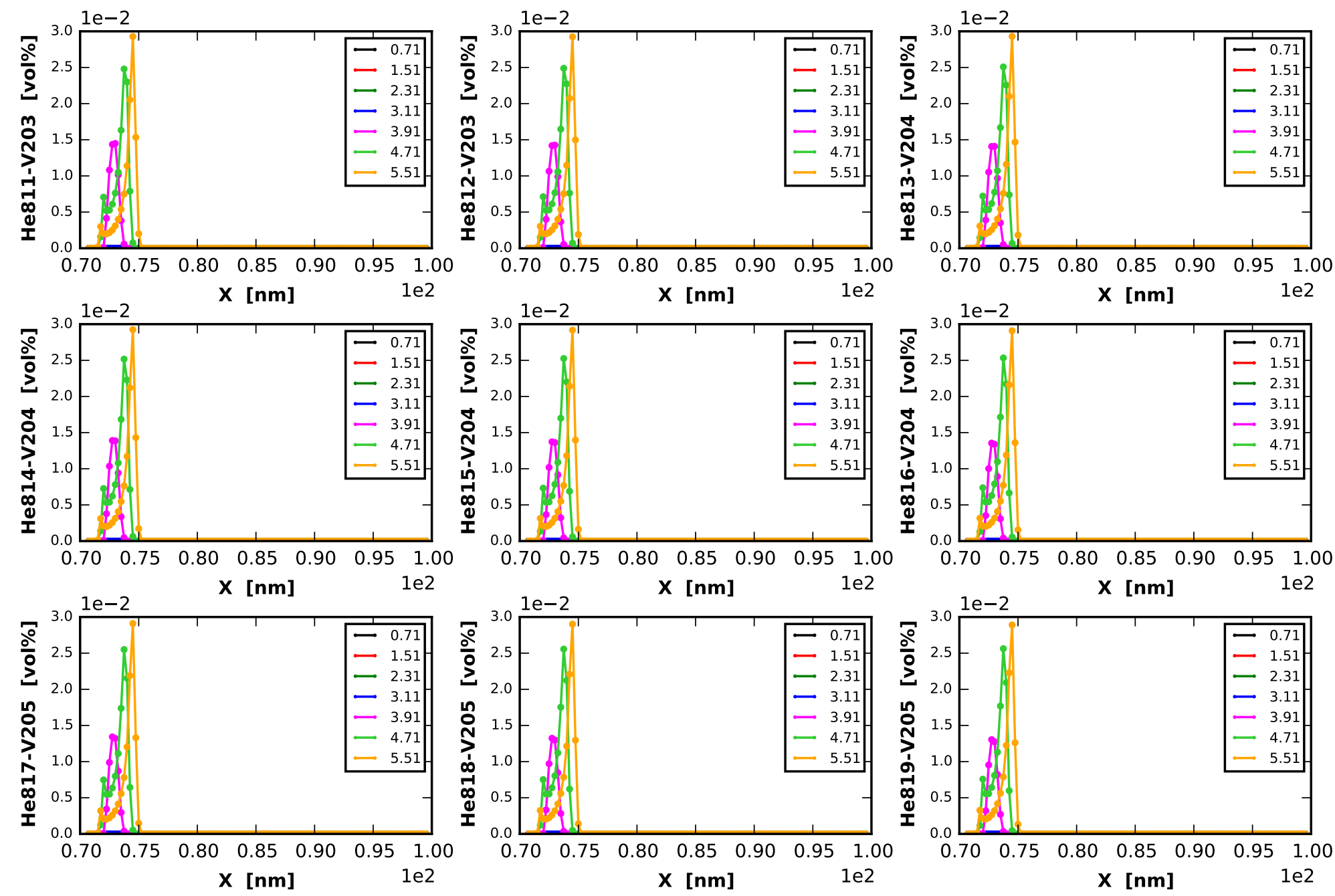
Transient: $[0.71,1.51,2.31,3.11,3.91,4.71,5.51]$ [us]
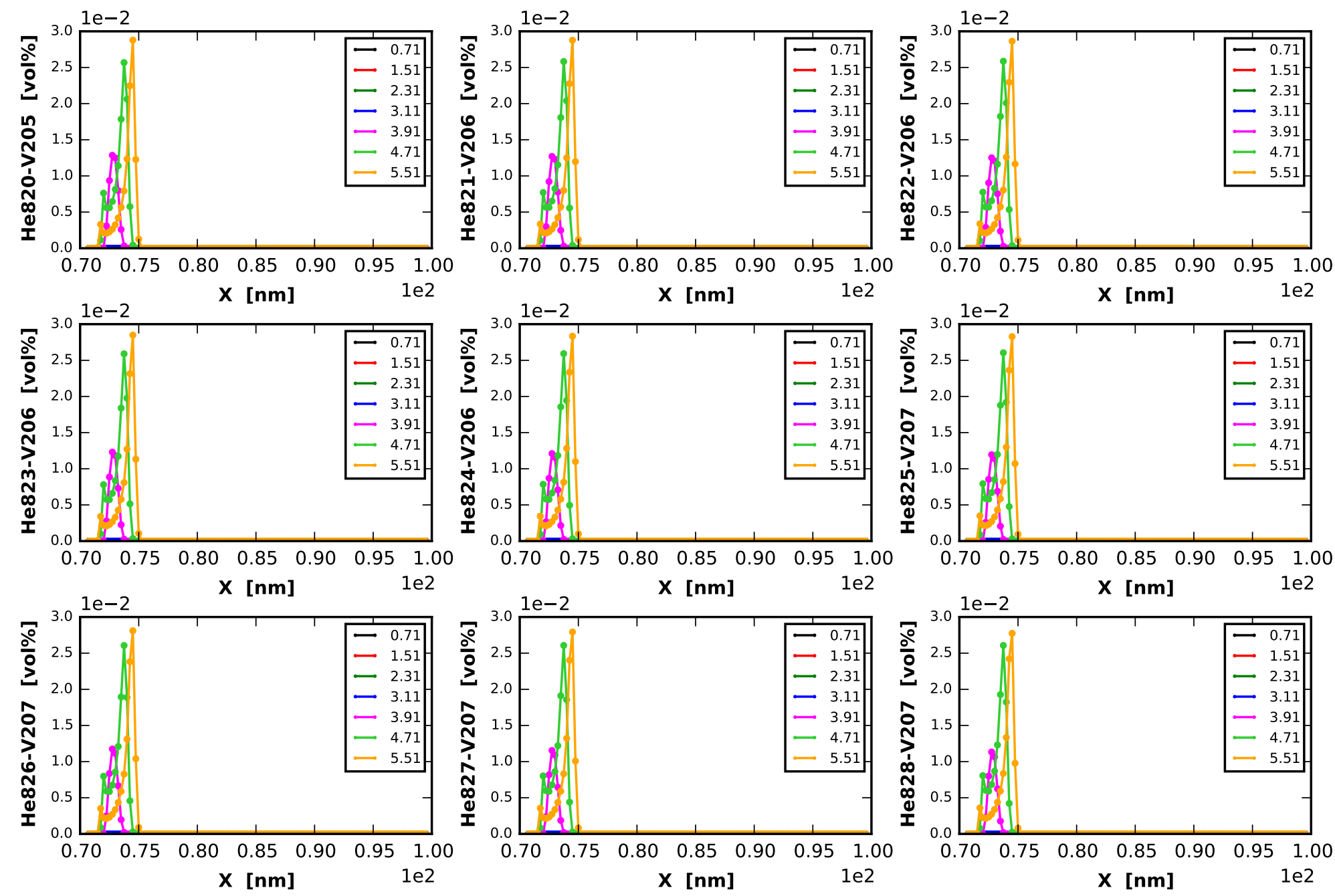
Transient: $[0.71,1.51,2.31,3.11,3.91,4.71,5.51]$ [us]
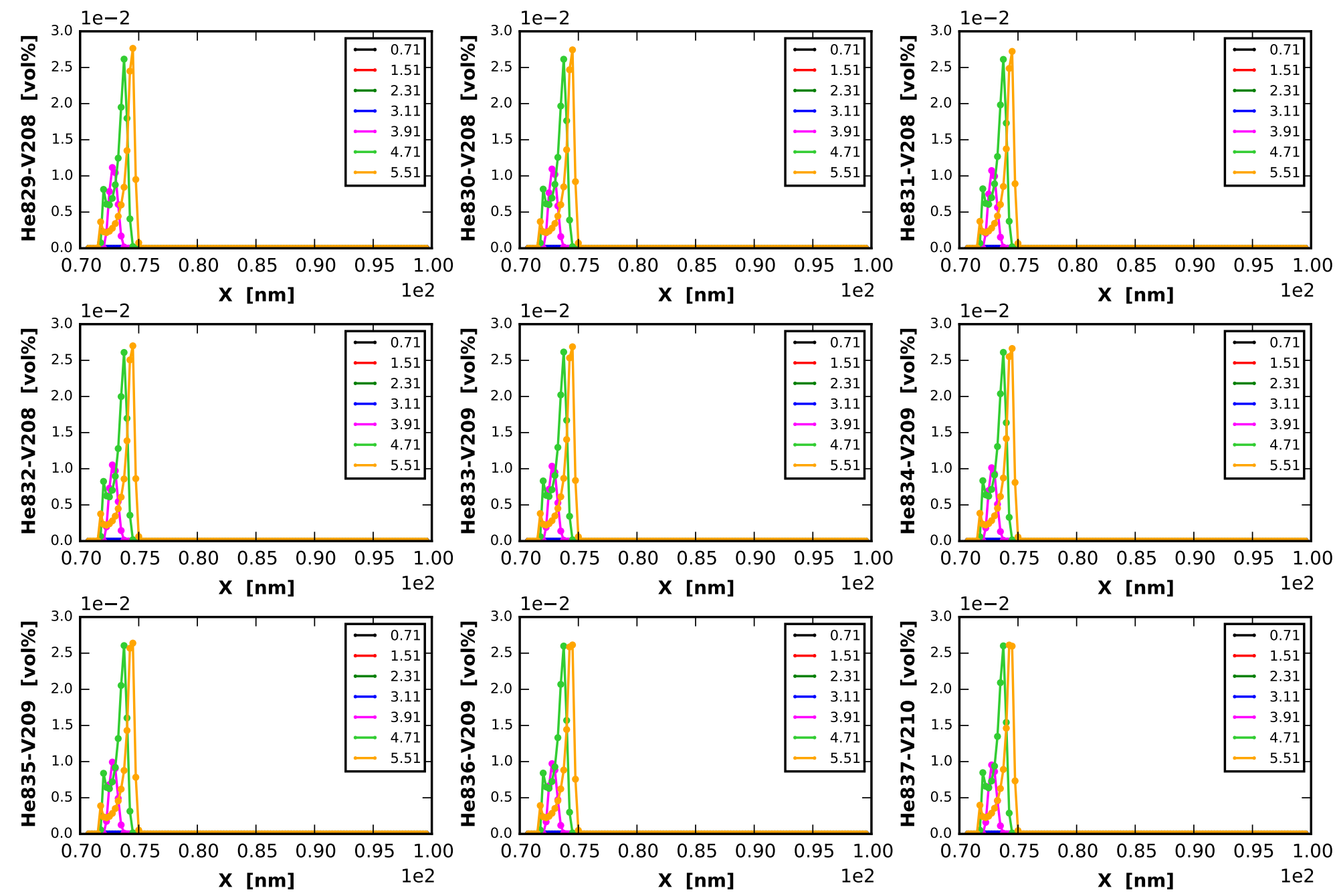
Transient: $[0.71,1.51,2.31,3.11,3.91,4.71,5.51]$ [us]
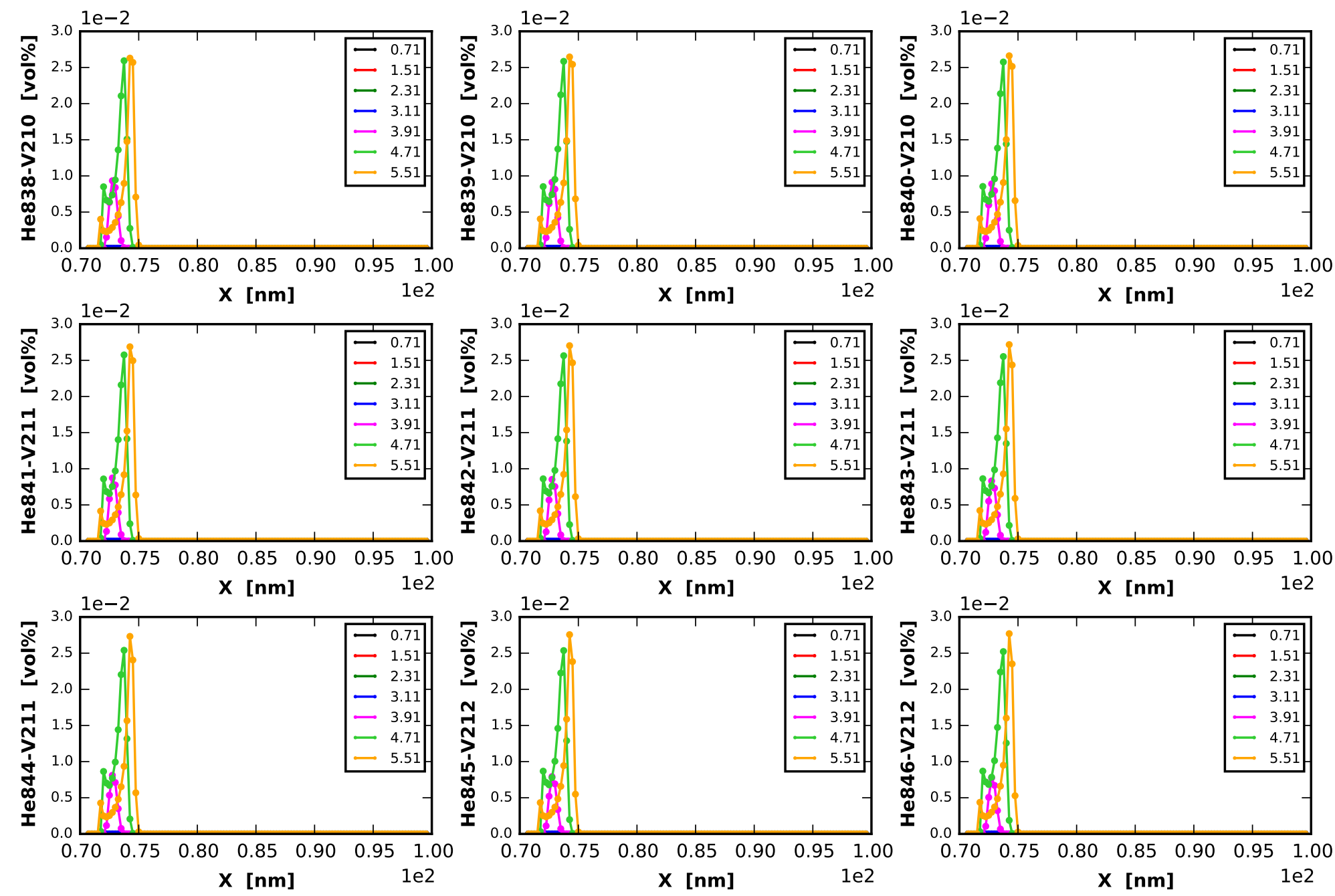
Transient: $[0.71,1.51,2.31,3.11,3.91,4.71,5.51]$ [us]
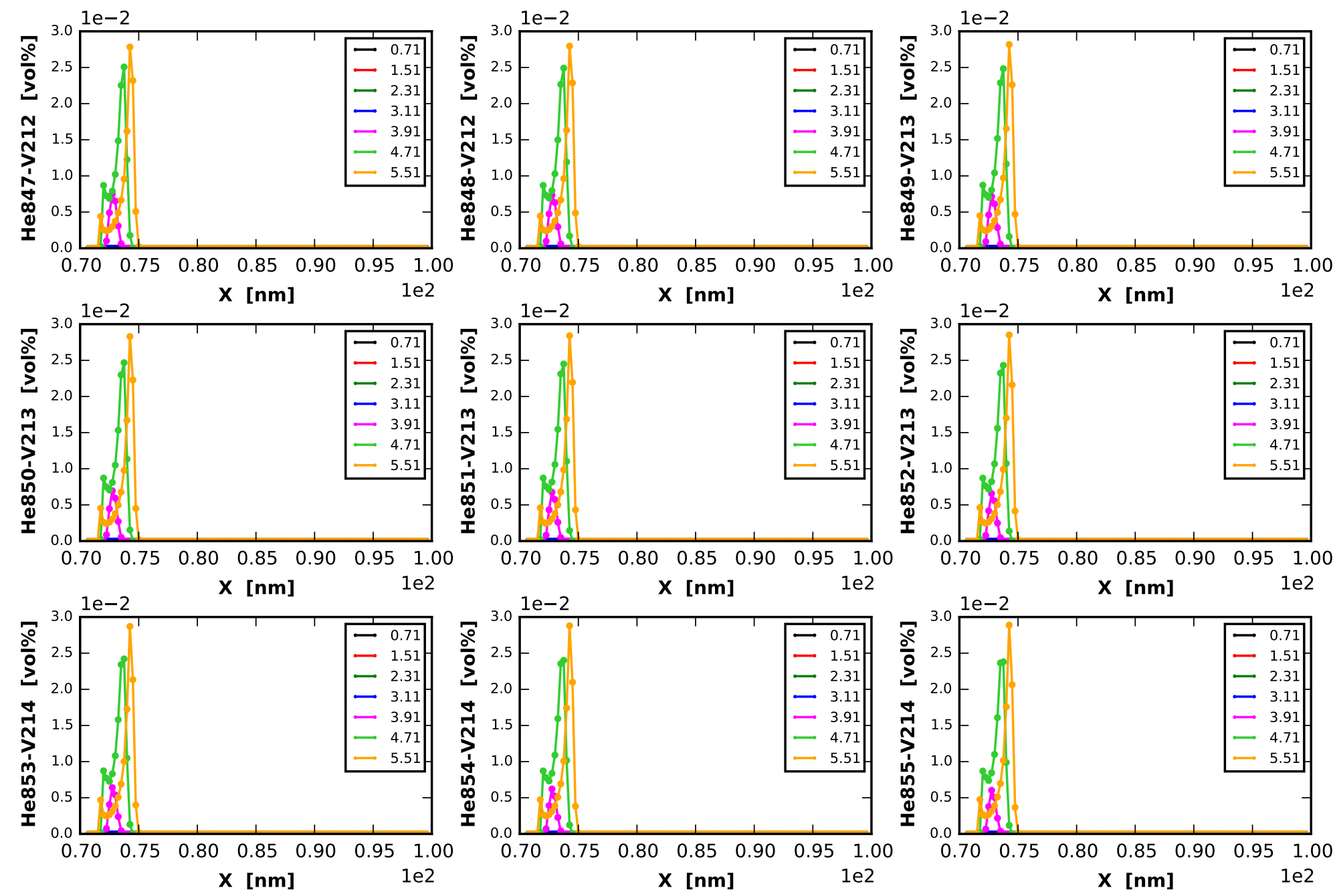
Transient: $[0.71,1.51,2.31,3.11,3.91,4.71,5.51]$ [us]
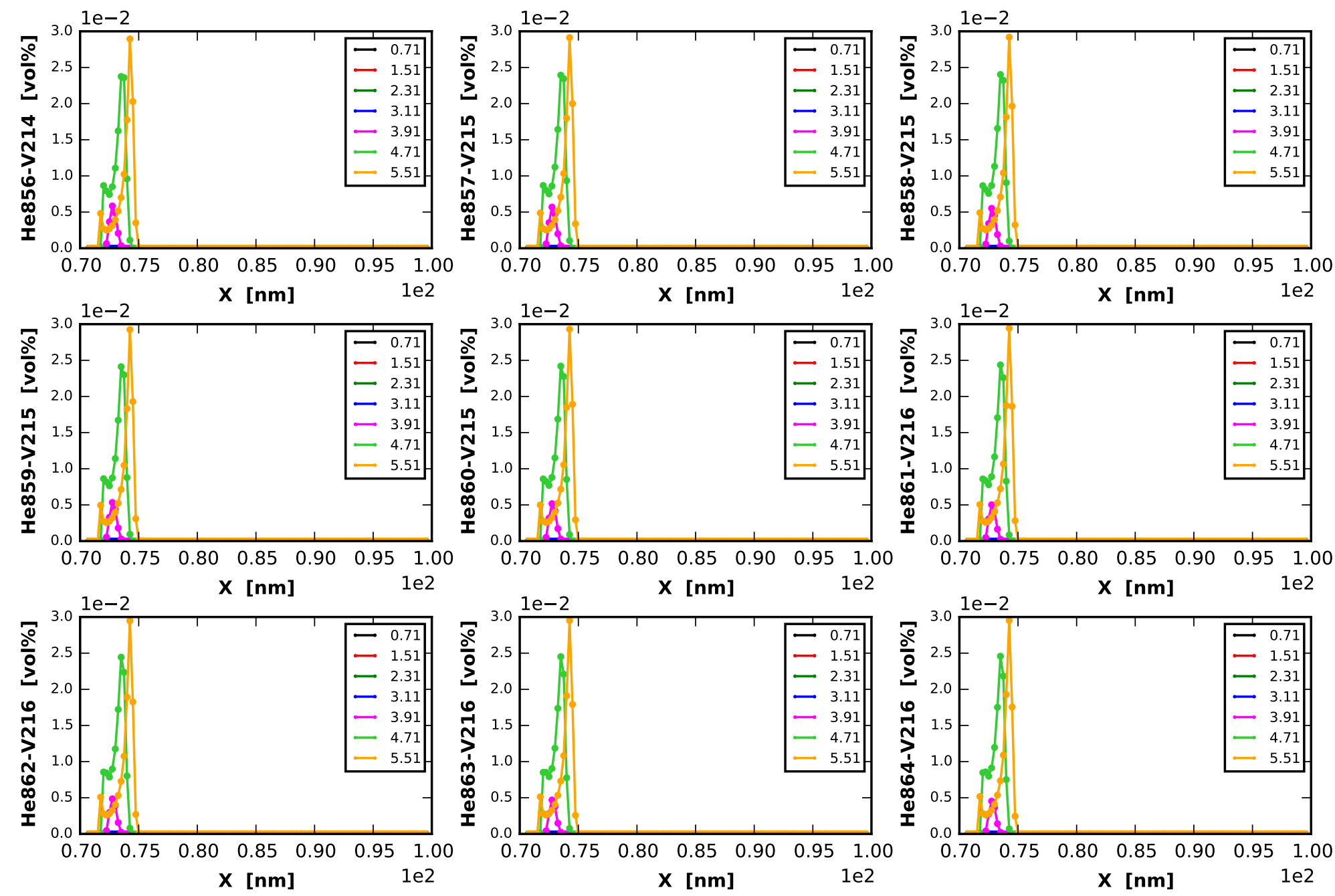
Transient: $[0.71,1.51,2.31,3.11,3.91,4.71,5.51]$ [us]
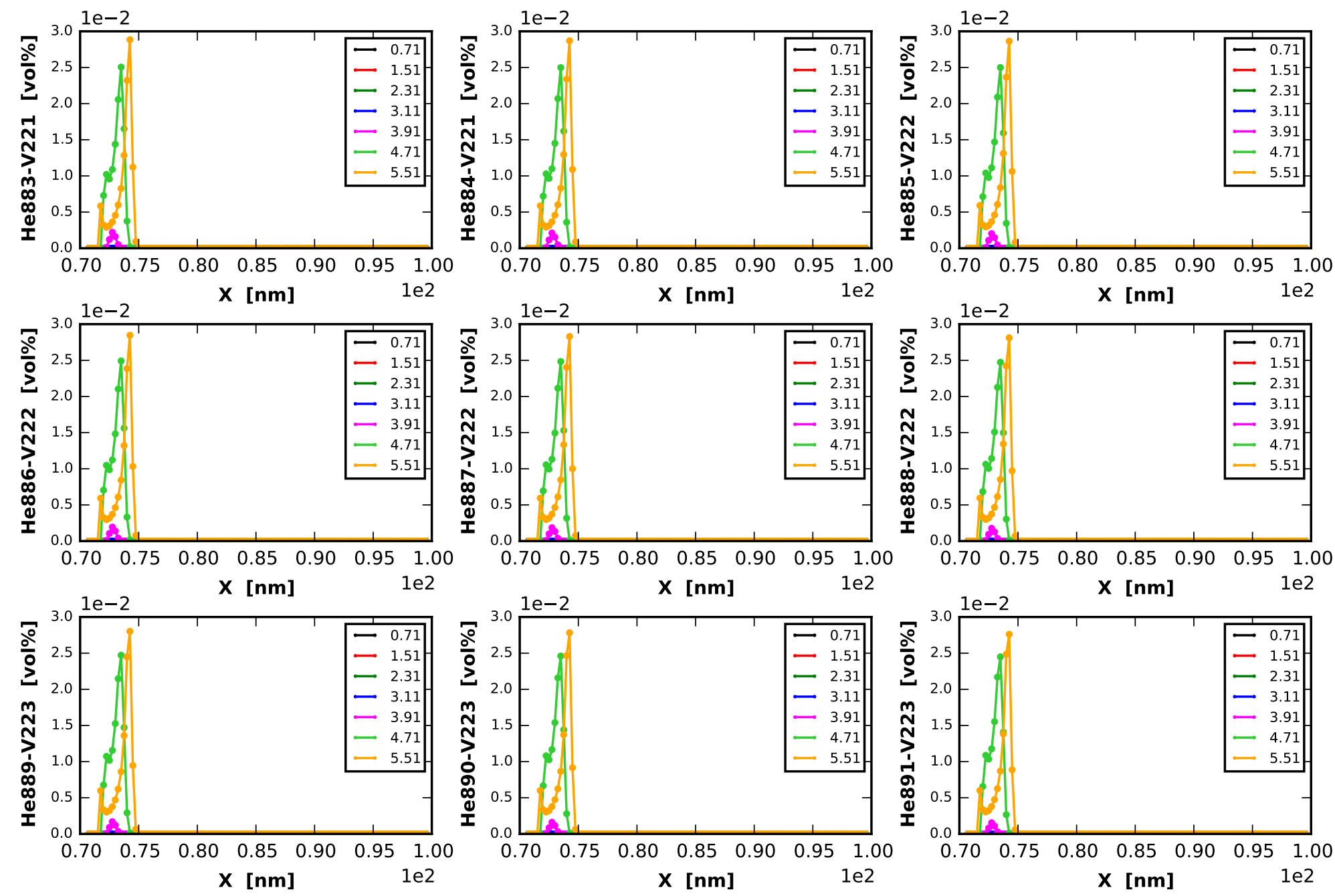
Transient: $[0.71,1.51,2.31,3.11,3.91,4.71,5.51]$ [us]
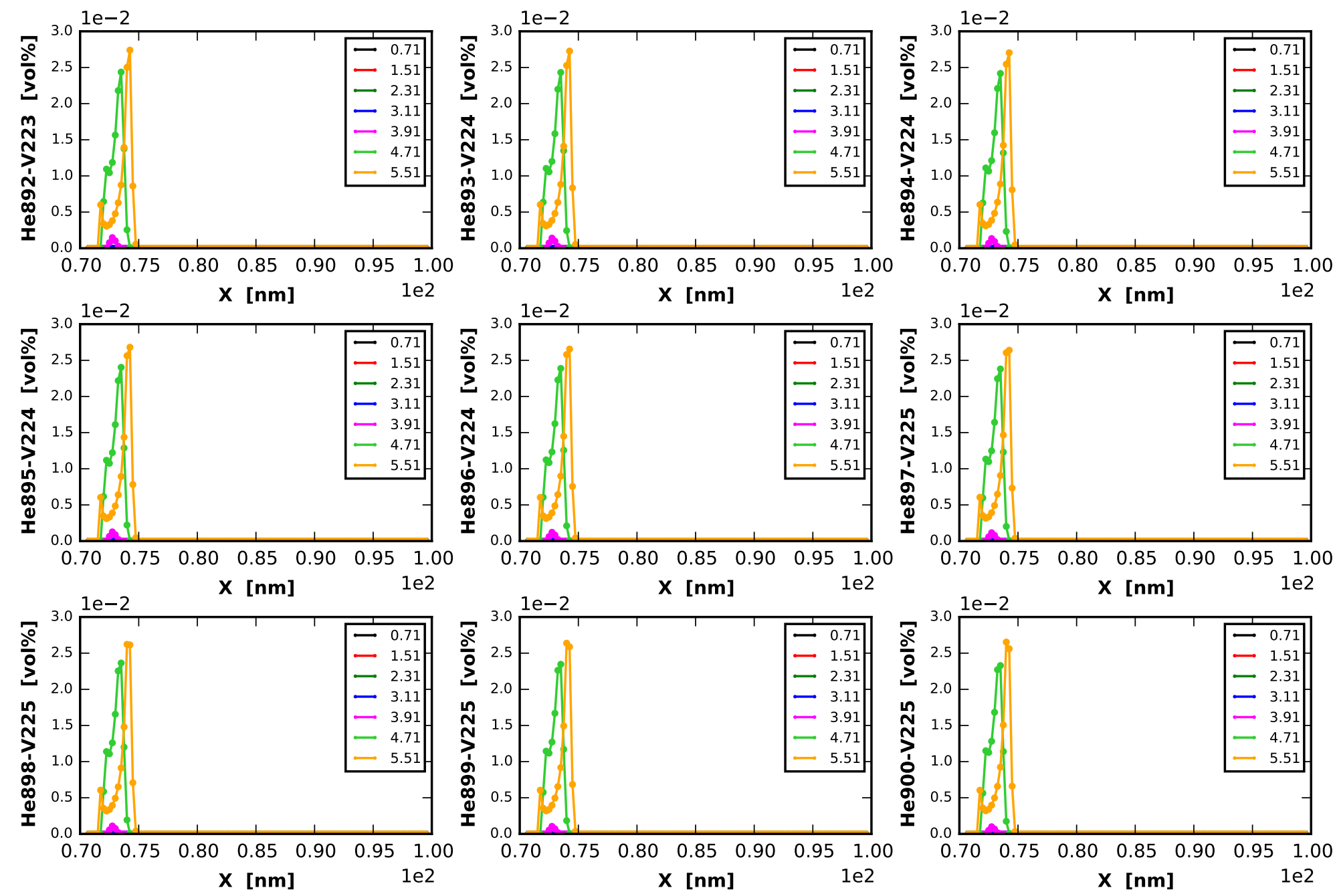
Transient: $[0.71,1.51,2.31,3.11,3.91,4.71,5.51]$ [us]
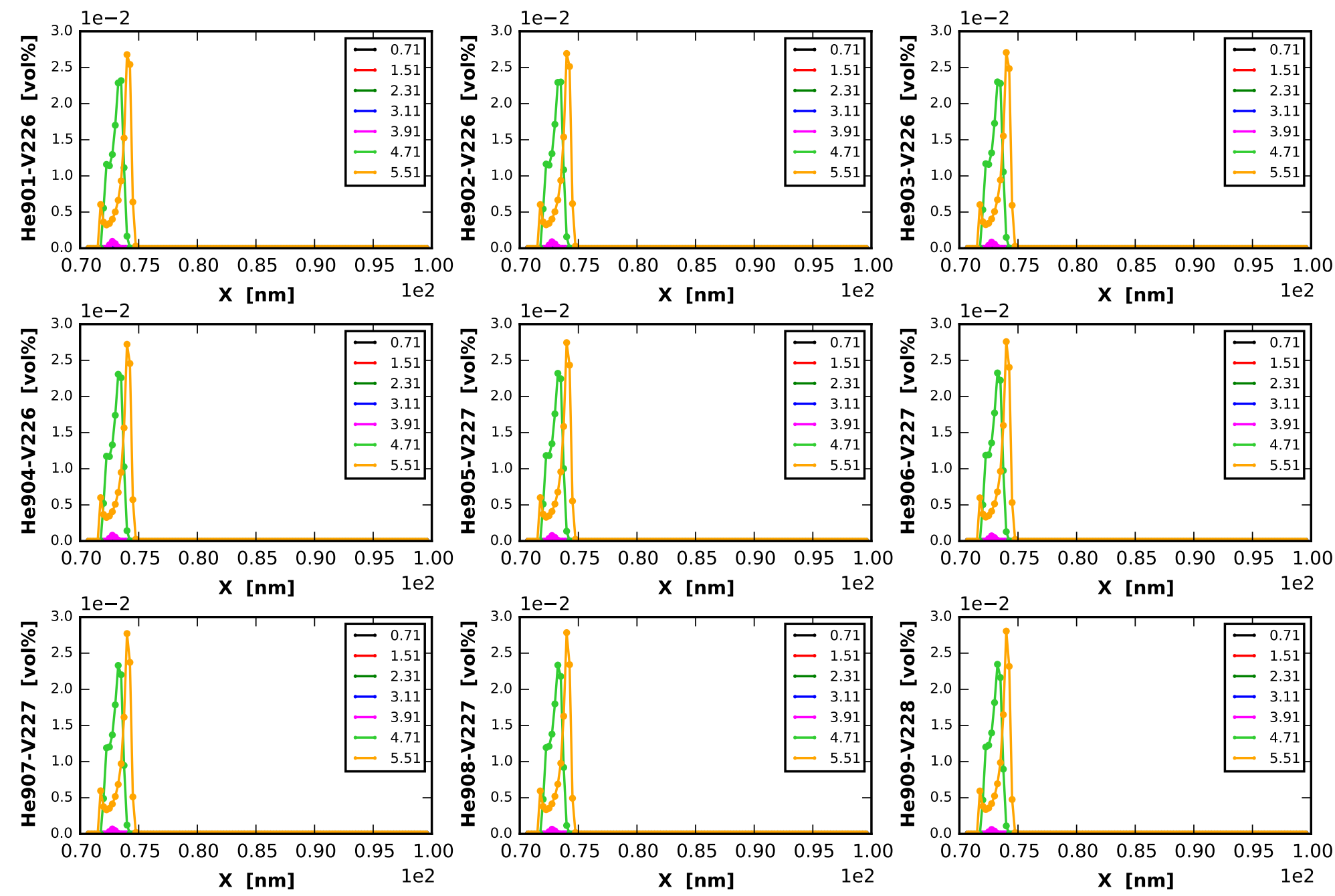
Transient: $[0.71,1.51,2.31,3.11,3.91,4.71,5.51]$ [us]
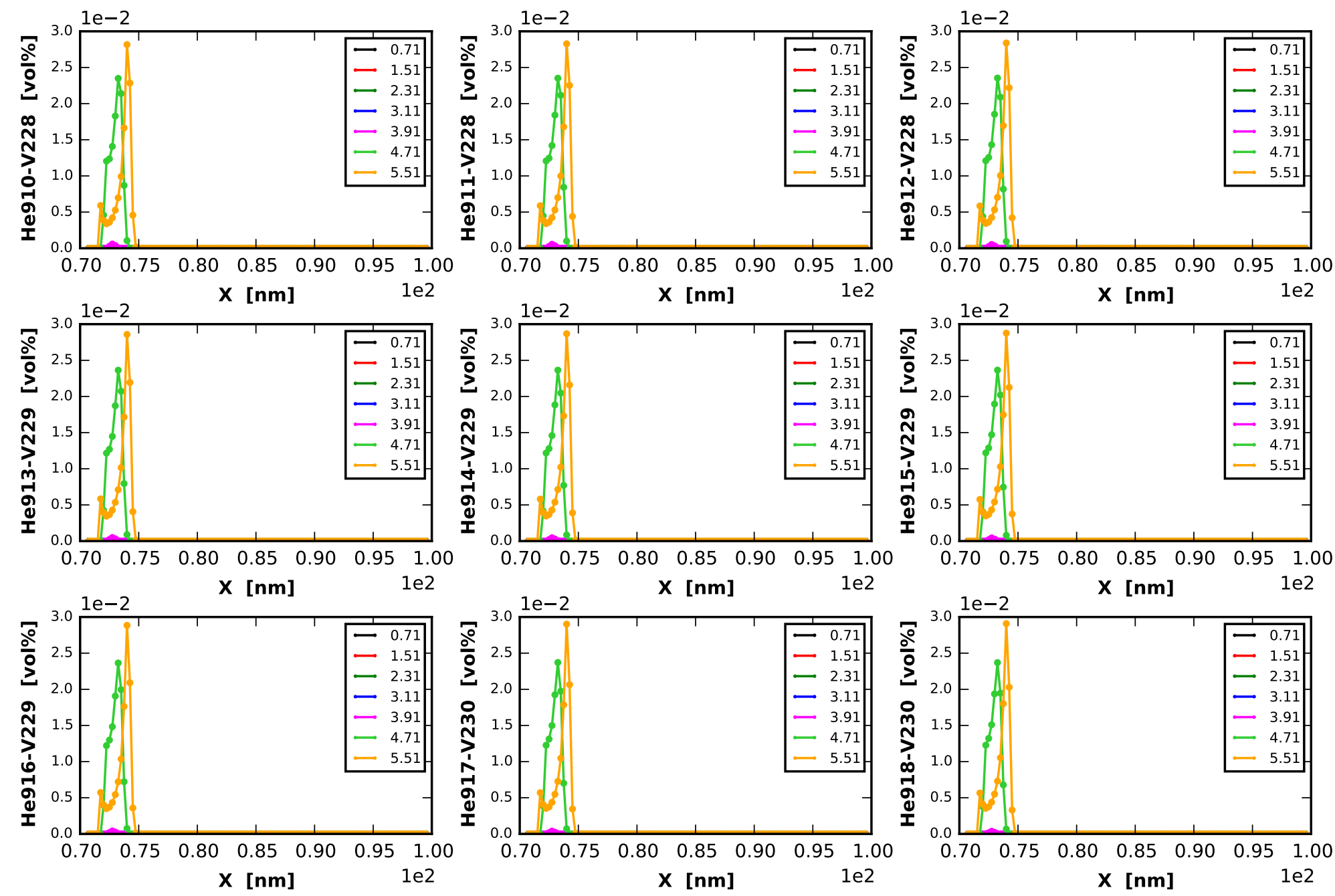
Transient: $[0.71,1.51,2.31,3.11,3.91,4.71,5.51]$ [us]
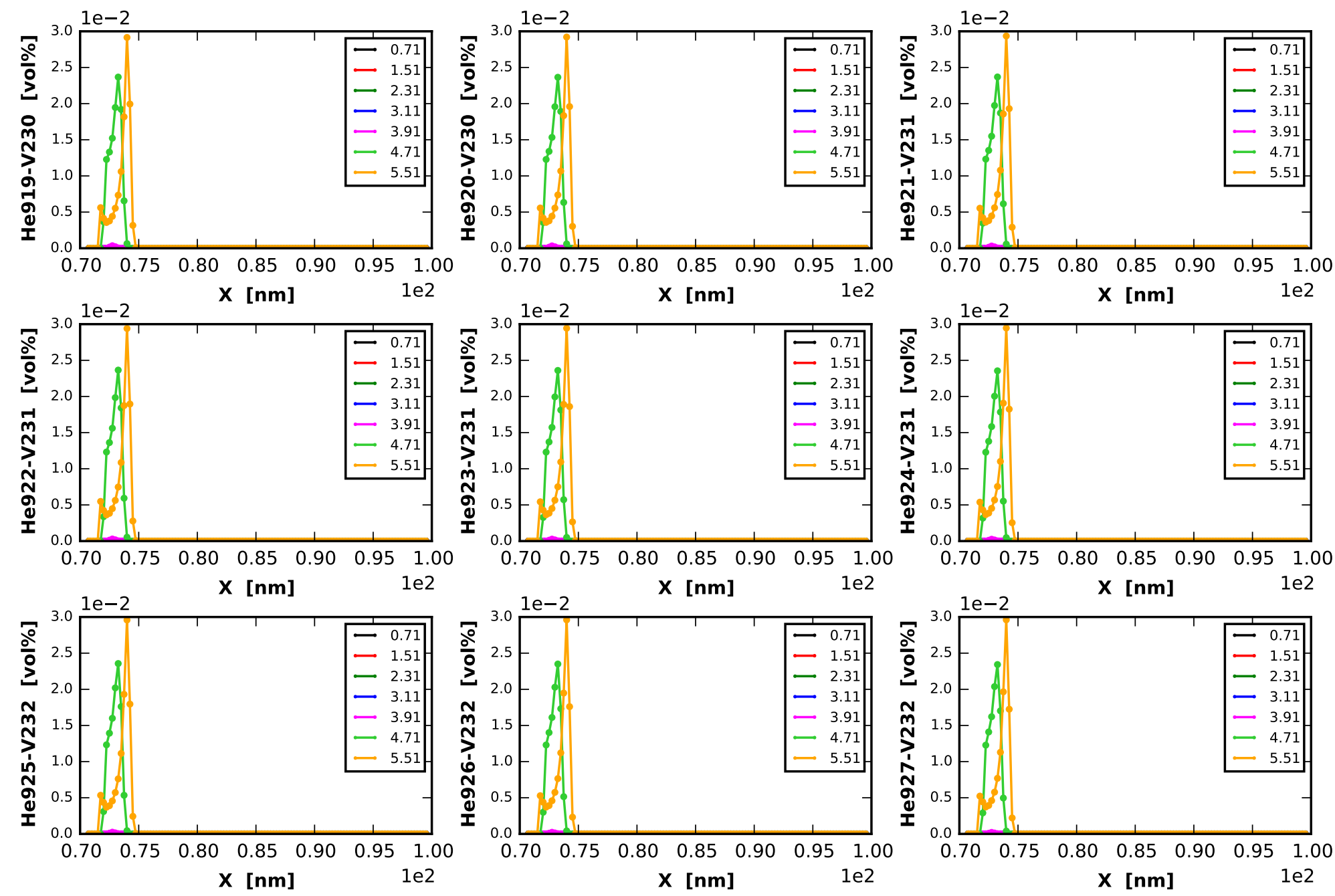
Transient: $[0.71,1.51,2.31,3.11,3.91,4.71,5.51]$ [us]
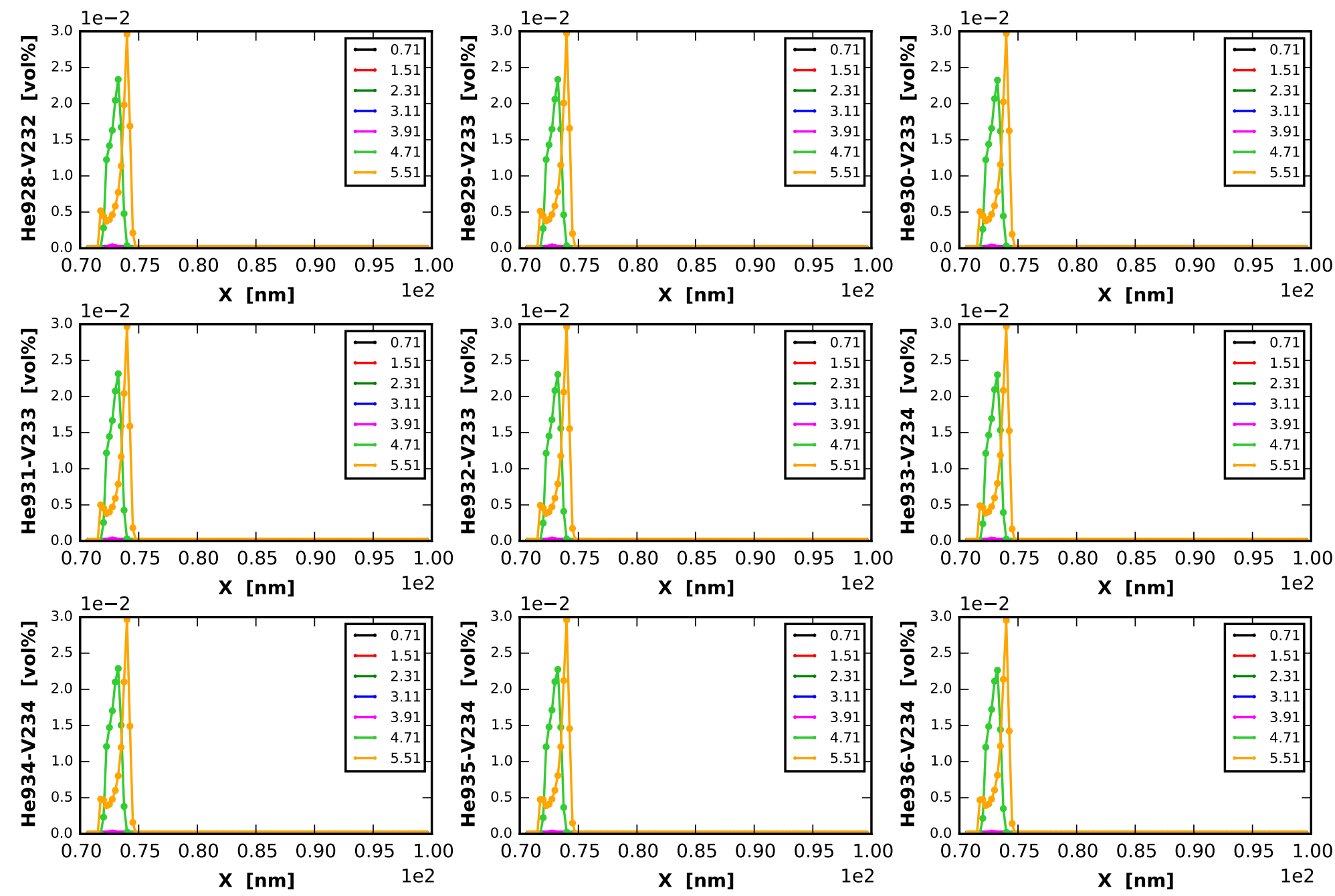
Transient: $[0.71,1.51,2.31,3.11,3.91,4.71,5.51]$ [us]
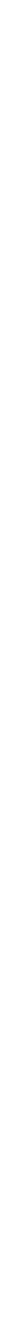
Transient: $[0.71,1.51,2.31,3.11,3.91,4.71,5.51]$ [us]
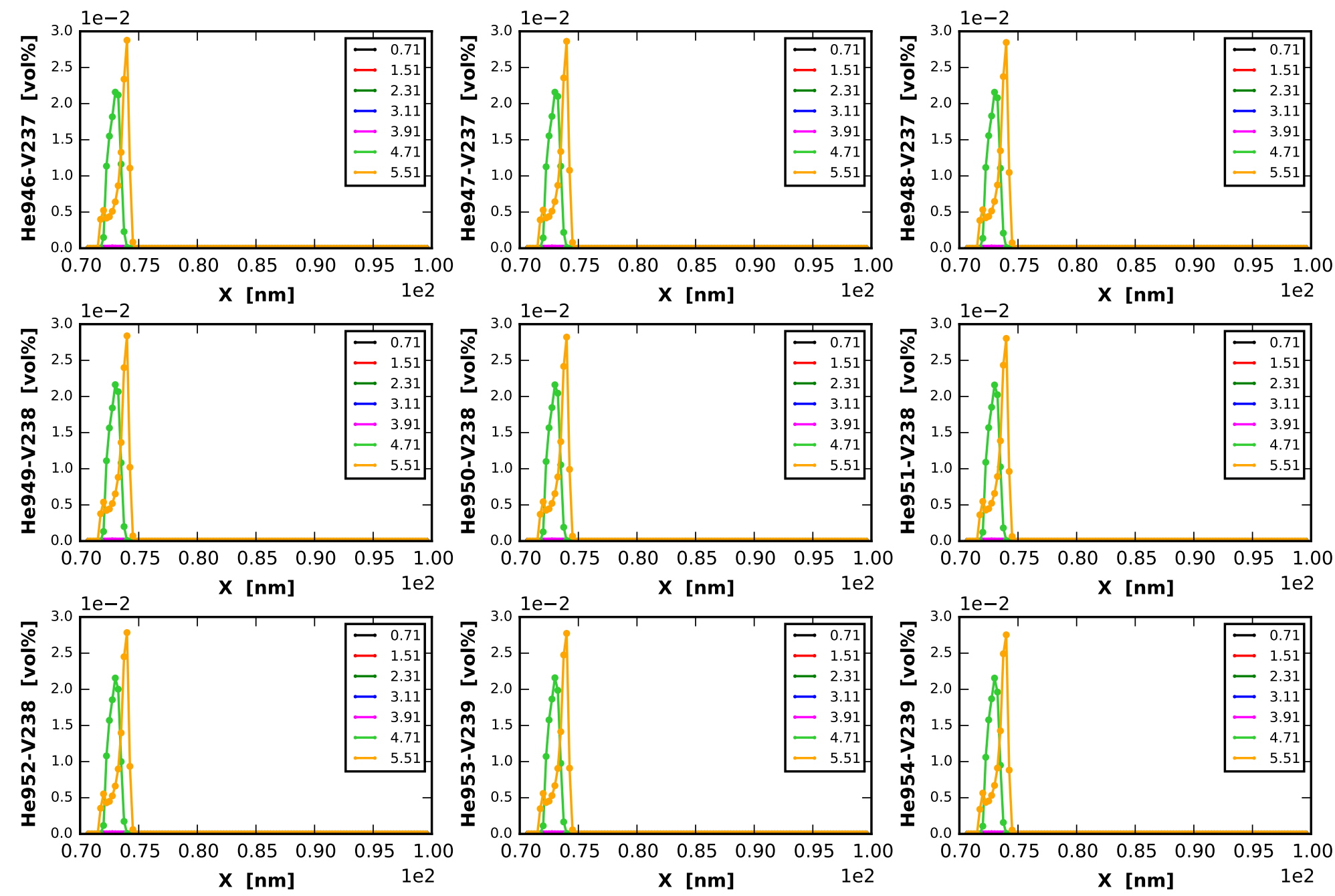
Transient: $[0.71,1.51,2.31,3.11,3.91,4.71,5.51]$ [us]
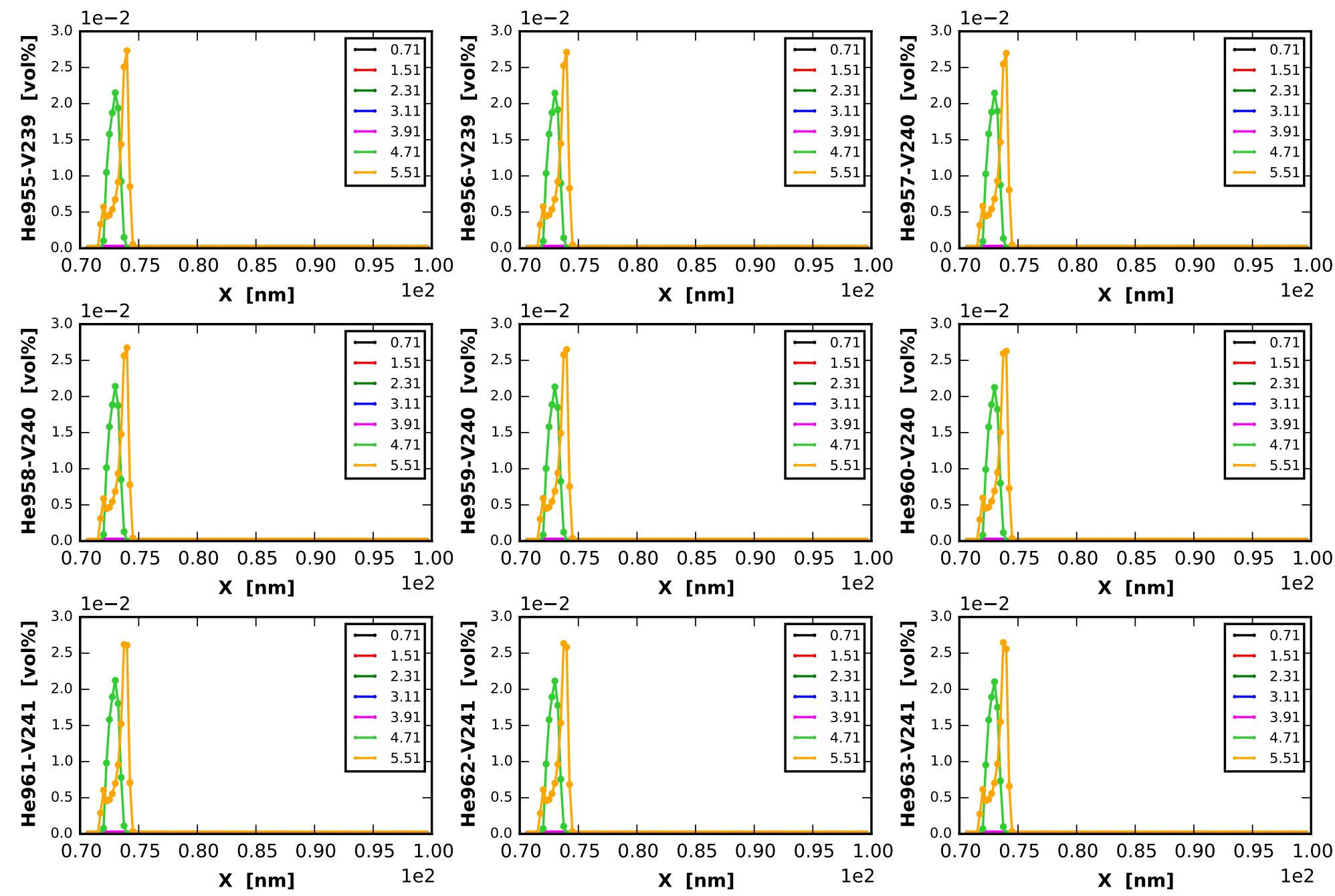
Transient: $[0.71,1.51,2.31,3.11,3.91,4.71,5.51]$ [us]
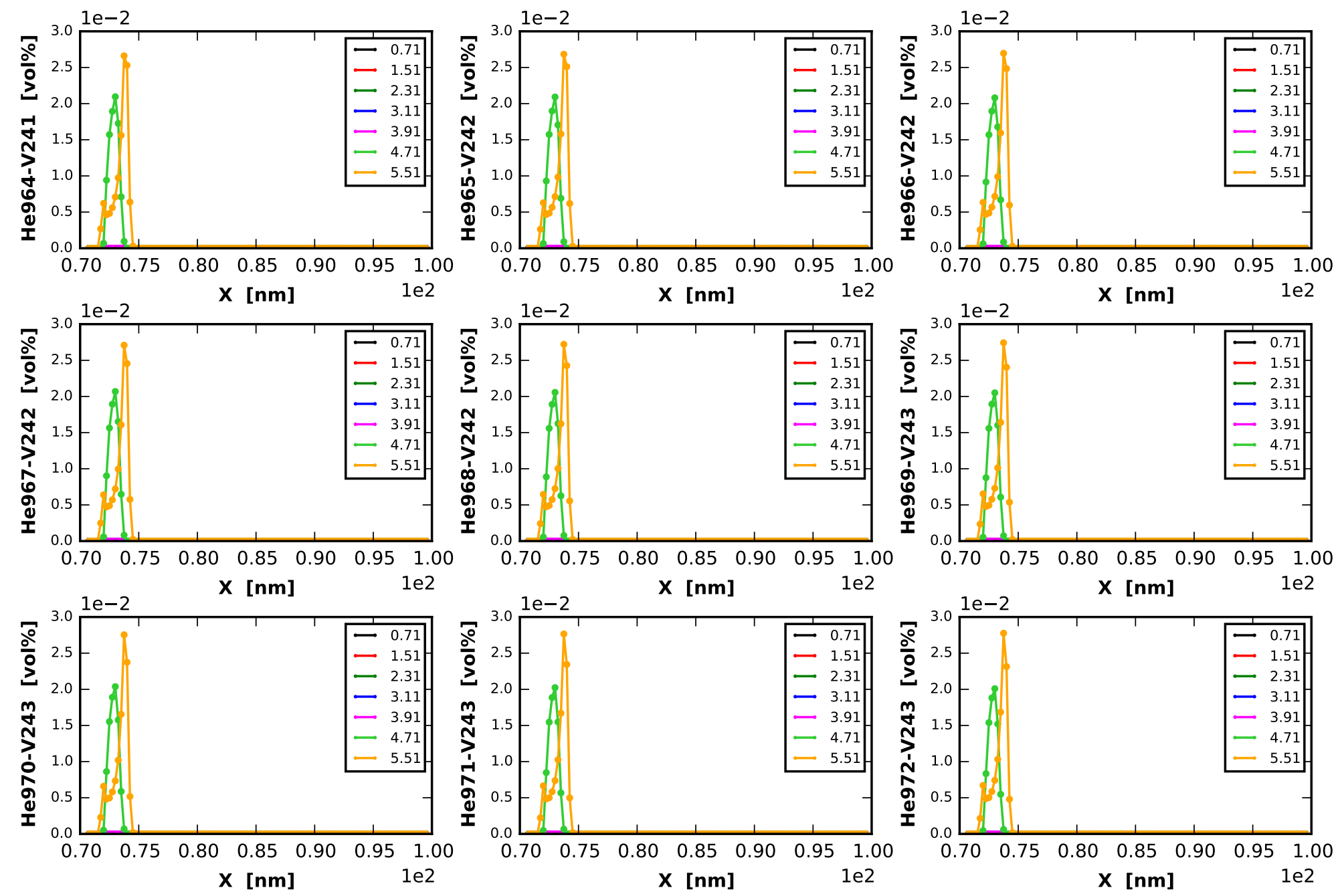
Transient: $[0.71,1.51,2.31,3.11,3.91,4.71,5.51]$ [us]
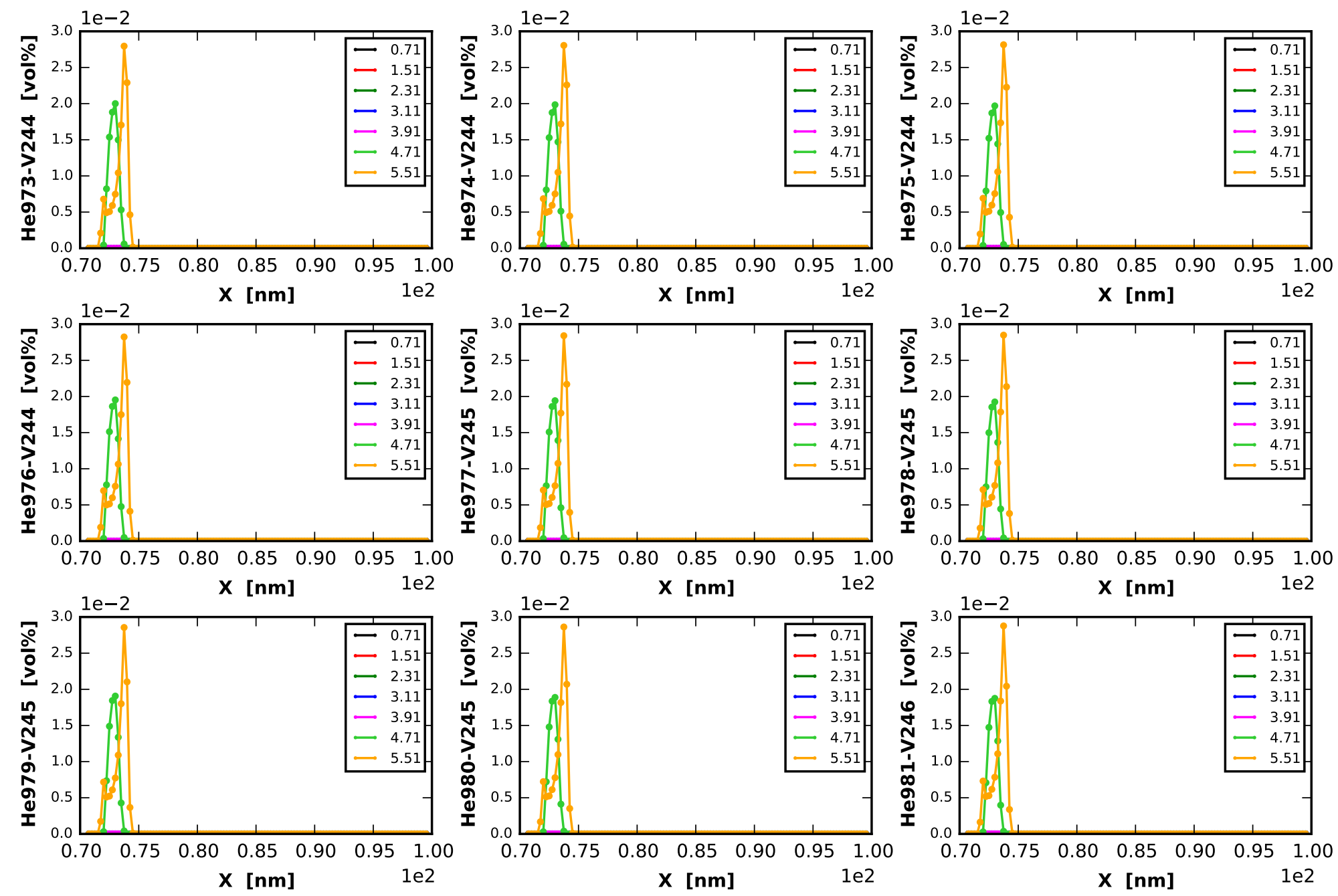
Transient: $[0.71,1.51,2.31,3.11,3.91,4.71,5.51]$ [us]
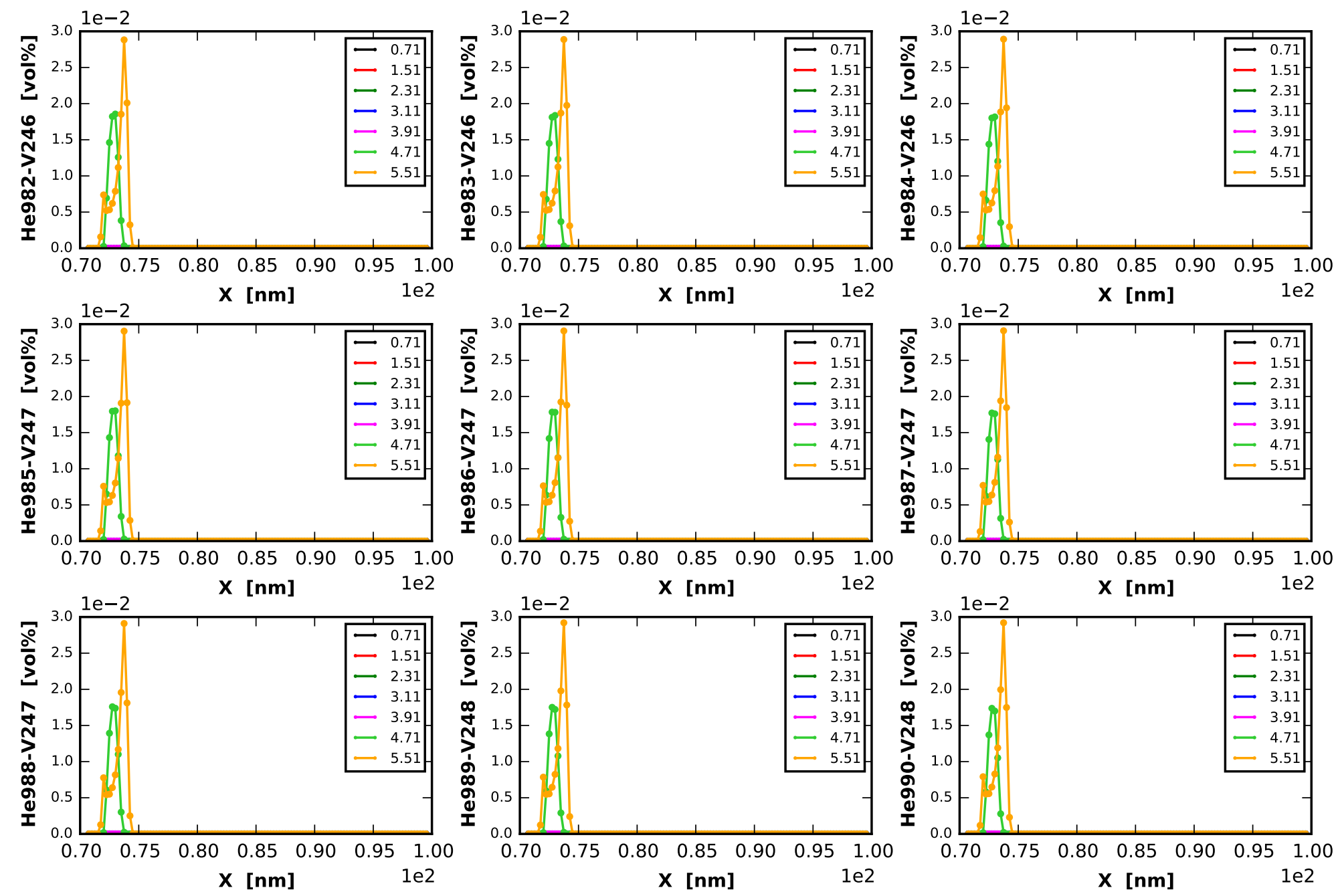
Transient: $[0.71,1.51,2.31,3.11,3.91,4.71,5.51]$ [us]
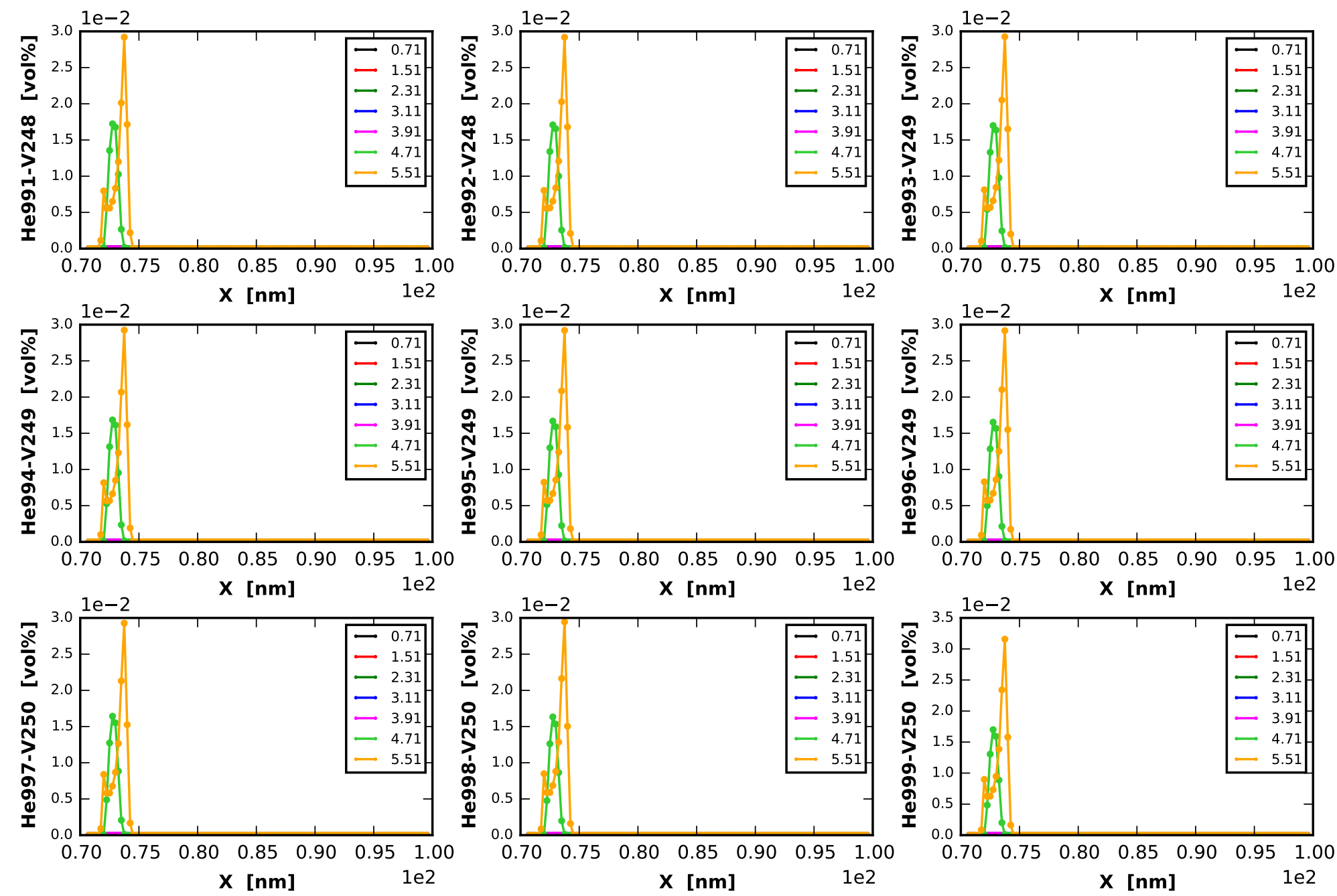


\section{Transient: [0.71, 1.51, 2.31, 3.11, 3.91, 4.71, 5.51] [us]}
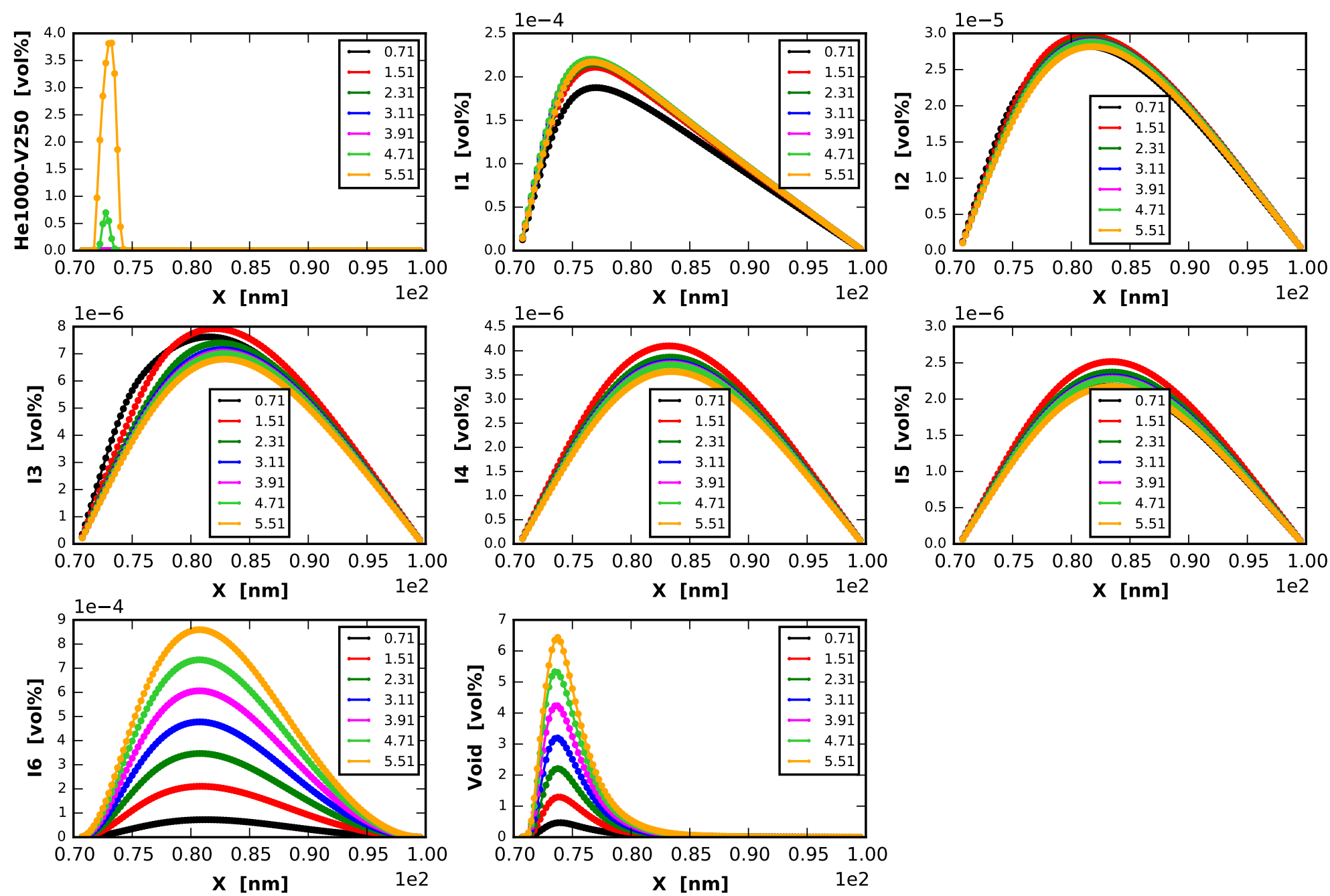


\section{Acknowledgments}

This material is based upon work supported by the U. S. Department of Energy, Office of Nuclear Energy and Office of Science, Office of Advanced Scientific Computing Research through the Scientific Discovery through Advanced Computing (SciDAC) project on Fission Gas Behavior. The Oak Ridge National Laboratory is managed and operated by UT-Battelle, LLC, under contract to the DOE (DE-AC05-00OR22725). 


\section{References}

Sophie Blondel, Karl D. Hammond, Lin Hu, Dimitrios Maroudas, and Brian D. Wirth. Modeling helium segregation to the surfaces of plasma-exposed tungsten as a function of temperature and surface orientation. Fusion Sci. Technol., 71:22-35, 2017.

Thibault Faney. Numerical Simulation of Tungsten under Helium Irradiation. Ph.D. dissertation, University of California, Berkeley, Department of Nuclear Engineering, 2013.

Yijing Hu, Qiangqiang Shi, Valmor F. de Almeida, and Xiaolin Li. Numerical simulation of phase transition problems with explicit interface tracking. Chem. Eng. Sci., 128:92-108, 2015.

Dimitrios Maroudas, Sophie Blondel, Lin Hu, Karl D. Hammond, and Brian D. Wirth. Helium segregation on surfaces of plasma-exposed tungsten. J. of Phys: Condens. Matter, 28(6):64004, 2016. 